\title{
HARUZO HIDA
}

\section{On the search of genuine $p$-adic modular $L$-functions for $G L(n)$. With a correction to : on $p$-adic $L$-functions of $G L(2) \times G L(2)$ over totally real fields}

Mémoires de la S. M. F. $2^{e}$ série, tome 67 (1996)

<http://www.numdam.org/item?id=MSMF_1996_2_67_R1_0>

(C) Mémoires de la S. M. F., 1996, tous droits réservés.

L'accès aux archives de la revue « Mémoires de la S. M. F. » (http://smf. emath.fr/Publications/Memoires/Presentation.html) implique l'accord avec les conditions générales d'utilisation (http://www.numdam.org/conditions). Toute utilisation commerciale ou impression systématique est constitutive d'une infraction pénale. Toute copie ou impression de ce fichier doit contenir la présente mention de copyright.

\section{Numdam}


Mémoire de la

Société Mathématique de France

Numéro 67, 1996

\title{
On the Search of Genuine $p$-adic Modular $L$-Functions for $G L(n)$
}

\author{
Haruzo HIDA*
}

\begin{abstract}
The purpose of this monograph is to state several conjectures concerning the existence and the meromorphy of many variable $p$-adic $L$-functions attached to many variable Galois representations (for example having values in $G L_{n}\left(\mathbb{Z}_{p}\left[\left[X_{1}, \ldots, X_{r}\right]\right]\right)$ ) and to present some supporting examples for the conjectures. Our discussion in the earlier sections is therefore quite speculative, but towards the end, we gradually make things more concrete.

Résumé - Le but de cette monographie est de formuler quelques conjectures concernant l'existence et la méromorphie des fonctions $L p$-adiques de plusieurs variables attachées à des représentations galoisiennes de plusieurs variables (par exemple, à valeurs dans $\left.G L_{n}\left(\mathbb{Z}_{p}\left[\left[X_{1}, \ldots, X_{r}\right]\right]\right)\right)$ et de présenter quelques exemples motivant nos conjectures. Nous commençons par une discussion assez spéculative, mais vers la fin, nous donnons des résultat plus concrets.
\end{abstract}

AMS Subject Classification Index: 11F13, 11F41, 11F67, 11F70, 11F85.

*Department of Mathematics, University of California, Los Angeles CA 90024-1555, U.S.A., e-mail: hida@math.ucla.edu 



\section{Contents}

1 Introduction $\quad 1$

2 p-Adic Hecke algebras $\quad 11$

2.1 The weight group . . . . . . . . . . . . . . . 11

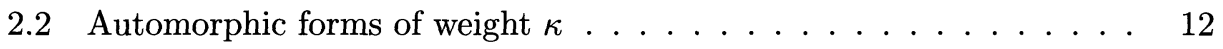

2.3 Fourier-expansion and rationality . . . . . . . . . . . . 14

$2.4 q$-Expansion, integrality and Hecke operators . . . . . . . . . . 14

2.5 -adic Hecke algebras . . . . . . . . . . . . . . . . 16

2.6 Duality between Hecke algebras and $p$-adic modular forms . . . . . 17

2.7 Modular parametrization by $\mathscr{X} \ldots \ldots . \ldots . \ldots 18$

2.8 Galois representations attached to $\lambda \ldots \ldots$. . . . . . . . . . . . . . . . . . . .

2.9 Congruence modules and differential modules . . . . . . . . . . . 21

2.10 Non-abelian class number formulas . . . . . . . . . . . . . 22

3 Periods of motives $\quad 29$

3.1 Periods of motives . . . . . . . . . . . . . . . 29

3.2 p-Adic periods of motives . . . . . . . . . . . . . . 32

3.3 An example of vanishing $p$-adic periods . . . . . . . . . . . . . 34

3.4 Non-vanishing of $p$-adic periods . . . . . . . . . . . . . . . . 35

3.5 Admissibility condition . . . . . . . . . . . . . . 38

4 Periods of arithmetic Galois representations $\quad 41$

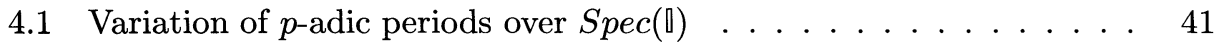

4.2 Genuine $p$-adic $L$-functions . . . . . . . . . . . . . . . 43

4.3 Normalized $p$-adic $L$-functions . . . . . . . . . . . . . . . . . . . . 44

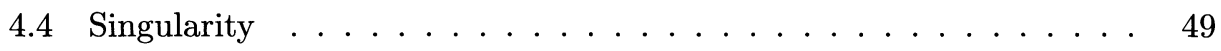

$4.5 \mu$-invariant of cyclotomic restrictions of $L_{S}(\varphi) \ldots \ldots \ldots$ 
4.6 Nearly ordinary $\llbracket$-adic representations . . . . . . . . . . . 51

4.7 Residually reducible case . . . . . . . . . . . . . . 53

5 Periods of tensor products of motives $\mathbf{5 5}$

5.1 Tensor products of motives . . . . . . . . . . . . 55

5.2 Periods of $M_{1} \otimes M_{2} \ldots \ldots \ldots \ldots$. . . . . . . . . . 56

5.3 Periods of $M_{1} \otimes \ldots \otimes M_{r} \ldots \ldots \ldots \ldots$. . . . . . . . 58

5.4 Conjectures on $\varphi_{1} \otimes \cdots \otimes \varphi_{r} \ldots \ldots \ldots \ldots \ldots$

5.5 The case of $\varphi_{1} \otimes \varphi_{2} \quad \ldots \ldots \ldots$. . . . . . . . . . 60

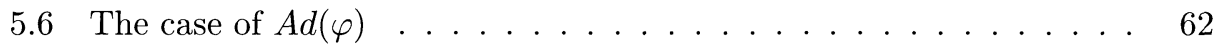

5.7 The case of $\varphi_{1} \otimes \varphi_{2} \otimes \varphi_{3} \ldots \ldots \ldots \ldots$. . . . . . . . . 62

6 -Adic Rankin products 65

6.1 Complex Rankin products . . . . . . . . . . . . . 65

6.2 The $p$-adic Rankin product in [41] . . . . . . . . . . . . 66

6.3 Ordinary versus nearly ordinary . . . . . . . . . . . . 67

6.4 Auxiliary $L$-functions . . . . . . . . . . . . . . . . . . 70

6.5 Ordinary $p$-adic Rankin products . . . . . . . . . . . . . 72

6.6 Deduction of Theorem 6.5.2 from Theorem 6.5.1 . . . . . . . . . 75

$6.7 p$-Ordinary adelic Eisenstein measure . . . . . . . . . . . . . 79

6.8 Convoluted measure . . . . . . . . . . . . . . 80

6.9 Convolution along $\lambda \ldots \ldots \ldots \ldots$. . . . . . . . . . . . . . . . . . . . . . . . . . . . 82

6.10 Proof of Theorem 6.5.1 . . . . . . . . . . . . . 84

$7 \quad p$-Adic Rankin products in partially CM case $\quad \mathbf{8 5}$

7.1 CM components of Hecke algebras . . . . . . . . . . . . . 85

7.2 Periods of $M\left(\lambda_{P}\right)$ of CM type . . . . . . . . . . . 86

7.3 Genuine $p$-adic Rankin products . . . . . . . . . . . . . . . 90

7.4 Totally CM Case . . . . . . . . . . . . . . . . 90

8 -Ordinary Katz $p$-adic $L$-functions $\quad 93$

$8.1 p$-Ordinary Eisenstein measures . . . . . . . . . . . . . . . . . 93

8.2 The $p$-ordinary Katz $p$-adic $L$-functions . . . . . . . . . . . . . . . 94

$8.3 p$-Ordinary $L$-function $L_{p}(\Phi) \ldots \ldots \ldots \ldots \ldots$

8.4 Another proof of Theorem 8.3.1 . . . . . . . . . . . . . . 97

8.5 Representations isogenous to $\Phi \ldots \ldots$. . . . . . . . . . 99

$\begin{array}{ll}\text { Bibliography } & 101\end{array}$ 
$\begin{array}{ll}\text { A Correction to [41] } & 107\end{array}$

B List of Symbols

109

SociÉté Mathématique DE France 



\section{Introduction}

The purpose of this monograph is to state several conjectures concerning the existence, a precise interpolation property and the meromorphy of many variable $p$ adic $L$-functions attached to many variable (irreducible) Galois representations (for example having values in $\left.G L_{n}\left(\mathbb{Z}_{p}\left[\left[X_{1}, \ldots, X_{r}\right]\right]\right)\right)$ and to present some supporting examples for the conjectures. Often in the non-abelian case, $p$-adic $L$-function is determined up to unit multiples. We would like to identify a precise interpolation property necessary to determine the $p$-adic $L$ uniquely. This is important to have a non-abelian generalization of classical class number formulas and limit formulas which gives a direct connection between the analytic $p$-adic $L$ and arithmetic objects. The theory of abelian $p$-adic $L$-functions is constructed out of a desire to better understand the class number formulas. Contrary to this, in the non-abelian case, it is ironic for us to be left in search of a $p$-adic $L$-function genuinely characterized by the Galois representation by which the generalized class number formula should be written down. Our discussion in the earlier sections is therefore quite speculative, but towards the end, we gradually make things more concrete.

Let us describe our idea. Let $p$ be a prime, and fix algebraic closures $\overline{\mathbb{Q}}$ of $\mathbb{Q}$ and $\overline{\mathbb{Q}}_{p}$ of $\mathbb{Q}_{p}$. Let $F$ be a finite extension of $\mathbb{Q}$. We write $I$ for the set of all embeddings of $F$ into $\overline{\mathbb{Q}}$. We will later fix an embedding $i_{p}: \overline{\mathbb{Q}} \rightarrow \overline{\mathbb{Q}}_{p}$ and take a field $K$ which is a finite extension of $\mathbb{Q}_{p}$ in $\overline{\mathbb{Q}}_{p}$ containing the image under $i_{p} \sigma$ for every $\sigma \in I$. Write 0 for the $p$-adic integer ring of $K$. We extend $\sigma$ to an isomorphism: $\bar{F} \cong \overline{\mathbb{Q}}$ which we denote again by $\sigma$. For each $p$-adic place $\mathfrak{B}$ of $F$, we write $\mathscr{G}_{F_{\mathfrak{B}}}$ for the Galois group $\operatorname{Gal}\left(\bar{F}_{\mathfrak{B}} / F_{\mathfrak{B}}\right)$, and if $\mathfrak{B}$ is induced from $i_{p} \sigma$, we identify it with the decomposition group at $\mathfrak{B}$ in $\mathscr{G}_{F}=\operatorname{Gal}(\bar{F} / F)$ via $i_{p} \sigma$. Let $T_{n}$ be the standard diagonal torus of $\operatorname{Res}_{\mathfrak{r} / \mathbb{Z}} G L(n)$ for the integer ring $\mathfrak{r}$ of $F$ which is split over $\mathbb{O}$. Then we consider a normal integral domain $\llbracket$ finite (but not necessarily flat) over the completed group algebra $\mathcal{O}\left[\left[T_{n}\left(\mathbb{Z}_{p}\right)\right]\right]$. We assume that $\mathcal{O}$ is integrally closed in $\mathbb{0}$. Each $\mathbb{O}$-algebra homomorphism $P: \mathbb{Q} \rightarrow K$ restricted to $\mathcal{O}\left[\left[T_{n}\left(\mathbb{Z}_{p}\right)\right]\right]$ induces a continuous character $\kappa(P)$ of $T_{n}\left(\mathbb{Z}_{p}\right) \rightarrow K^{\times}$. We call $P \in \operatorname{Spec}(\square)(K)$ arithmetic if it induces a weight (that is, an algebraic character) of the torus $T_{n}$ 
on an open neighborhood of the identity in $T_{n}\left(\mathbb{Z}_{p}\right)$. We want to study a continuous Galois representation $\varphi: \mathscr{G}_{F} \rightarrow G L_{n}(\mathbb{\square})$ acting on $V\left(\cong \mathbb{\square}^{n}\right)$ satisfying the following condition:

(A1) There are arithmetic points $P$ densely populated in $\operatorname{Spec}(\mathbb{})(K)$ such that the Galois representation $\varphi_{P}=P \circ \varphi$ is the p-adic étale realization of a rank $n$ pure motive $M_{P}$ defined over $F$ with coefficients in a number field $E_{P}$ in $\overline{\mathbb{Q}}$.

The number field $E_{P}$ depends on $P$, and the composite $E_{\infty}$ of all $E_{P}$ for $P$ satisfying (A1) is usually an infinite extension of $\mathbb{Q}$. We call points $P$ satisfying (A1) motivic. Densely populated motivic points determine an isomorphism $i_{p}: E_{\infty} \hookrightarrow \overline{\mathbb{Q}}_{p}$ such that $\operatorname{Tr}\left(\varphi_{P}\left(F r o b_{\mathfrak{l}}\right)\right)$ for primes $\mathfrak{I}$ unramified for $\varphi_{P}$ generates a subfield of $i_{p}\left(E_{P}\right)$. We extend $i_{p}$ to $\overline{\mathbb{Q}}$. To get reasonable $p$-adic $L$-functions, we need to assume further $\left(\mathrm{A} 2_{ \pm}\right)$For a dense subset of motivic points $P$, we have

(i) The Tate twist $M_{P}(1)$ is critical in the sense of Deligne [15], and for each p-adic place $\mathfrak{B}$ of $F$, the restriction of $\varphi_{P}$ to $\mathscr{G}_{F_{\mathfrak{\beta}}}$ is of Hodge-Tate type;

(ii) Writing $\mathscr{F}_{P}^{\mp}$ for the middle terms of the Hodge filtration of $H_{D R}\left(M_{P}\right)$ as in [15], for each p-adic place $\mathfrak{P}=i_{p} \sigma$ of $F$, there exists an $\square$ direct summand $V_{\mathfrak{B}}^{ \pm} \subset V$ (independent of $P$ ) stable under $\mathscr{G}_{F_{\mathfrak{\beta}}}$ such that the comparison isomorphism of Faltings [21] induces: $V_{\mathfrak{B}}^{\mp} \otimes_{\mathbb{1}, P} B_{H T} \cong$ $\mathscr{F}_{P}^{\mp} \otimes_{F \otimes E, i_{p} \sigma \otimes i_{p}} B_{H T}$,

where $B_{H T}$ is one of the Fontaine's rings [22] given as follows: Writing $\Omega$ for the $p$-adic completion of $\overline{\mathbb{Q}}_{p}$, we have $B_{H T} \cong \Omega\left[t, t^{-1}\right]$ on which $\mathscr{G}_{\mathbb{Q}_{p}}$ acts via the natural action on $\Omega$ and via the cyclotomic character on the indeterminate $t$. Since $V\left(\varphi_{P}\right)$ is of Hodge-Tate type, as a $\mathscr{G}_{F_{\mathfrak{B}}}$-module, writing $E_{P, \mathfrak{p}}$ for the topological closure of $i_{p}\left(E_{P}\right)$ in $\Omega, V\left(\varphi_{P}\right) \otimes_{E_{P, \mathfrak{p}}} \Omega \cong \bigoplus_{1 \leq i \leq n} \Omega\left(m_{i}\right)$ for integers $m_{i}$ depending on $i_{p} \sigma$, where $\Omega(m)=\Omega t^{m}$ as a graded component of $B_{H T}$. We call $\left(m_{1}, \ldots, m_{n}\right)$ the Hodge-Tate twist of $V\left(\varphi_{P}\right)$ at $\sigma$. We will show that the condition (ii) is equivalent to the admissibility condition of Panchishkin ([56] Section 5) for $M_{P}$ if $M_{P}$ is crystalline at $p$. A similar condition is stated and studied in [28] p.217 in terms of Selmer groups. In particular, the existence of $V_{\mathfrak{B}}^{+}$in $\left(\mathrm{A} 2_{-}\right)$plays an important role in defining the Selmer group. We require by (ii) an analytic coherence of $\mathscr{F}_{P}^{ \pm}$ with respect to analytically varying $P$. The density assumption included in $\left(\mathrm{A} 2_{ \pm}\right)$ about motivic critical points is important to guarantee the uniqueness of the $p$-adic $L$-function. We also assume

(unr) $\varphi$ is unramified at almost all places of $F$.

The (conjectural) n-adic representations arising from cohomological modular forms on $G L(n)_{/ F}$ further satisfies an additional condition besides (A1-2 \pm ). Since $T_{n} \cong$ $\mathbf{G}_{m}^{n}$ over $F, X\left(T_{n}\right)$ is isomorphic to the product of $n$ copies of the free module $\mathbb{Z}[I]$ 
generated by the set $I$ of all embeddings of $F$ into $\overline{\mathbb{Q}}$. Thus we can associate to each arithmetic point $P$, an $n$-tuple $\left(m_{1}(P), \ldots, m_{n}(P)\right)$ of elements in $\mathbb{Z}[I]$. Then the condition is

(A3) The Hodge-Tate twist of $\varphi_{P}$ restricted to $\mathscr{G}_{F_{\mathfrak{B}}}$ for the p-adic place $\mathfrak{P}$ induced by $i_{p} \sigma$ is given by $\left(m_{1}(P)_{c \sigma}, m_{2}(P)_{c \sigma}, \ldots, m_{n}(P)_{c \sigma}\right)$ for each $\sigma \in I$,

where for $m \in \mathbb{Z}[I]$, we have written $m=\sum_{\sigma} m_{\sigma} \sigma$, and $c$ stands for the complex conjugation which we specify below choosing an embedding $i_{\infty}$ of $\overline{\mathbb{Q}}$ into $\mathbb{C}$. This last condition (A3) might be always satisfied by representations satisfying (A1-2 \pm ) after modifying the algebra structure over $\mathcal{O}\left[\left[T_{n}\left(\mathbb{Z}_{p}\right)\right]\right]$ and is equivalent to $\left(\mathrm{A} 3^{\prime}\right)$ The Hodge types of $H_{B}\left({ }^{i_{\infty} \sigma} M_{P}\right) \otimes_{E, i_{\infty}} \mathbb{C}$ is given by $\left\{\left(m_{i}(P)_{\sigma}, m_{n+1-i}(P)_{c \sigma}\right)\right\}_{i}$, where $H_{B}\left({ }^{i_{\infty} \sigma} M_{P}\right)$ is the Betti realization of $M_{P}$ at $i_{\infty} \sigma$. Anyway, we call the representation satisfying (A1-3) and (unr) an arithmetic Galois representation.

As conjectured by Deligne [15], for each motivic point $P$ as in (A2_), we expect to have a well defined special value

$$
\frac{L\left(1, M_{P}\right)}{\mathbf{c}^{+}\left(M_{P}(1)\right)} \in E_{P} \subset E_{P} \otimes_{\mathbb{Q}} \mathbb{C}
$$

for Deligne's period $\mathbf{c}^{+}\left(M_{P}(1)\right)$ in $\left(E \otimes_{\mathbb{Q}} \mathbb{C}\right)^{\times}$. We now choose an embedding $i_{\infty}: \overline{\mathbb{Q}} \hookrightarrow \mathbb{C}$ and write $\frac{L_{i_{\infty}}\left(1, M_{P}\right)}{c_{\infty, i_{\infty}}^{+}\left(M_{P}(1)\right)}$ for the $i_{\infty}$-component of $\frac{L\left(1, M_{P}\right)}{\mathbf{c}^{+}\left(M_{P}(1)\right)}$. An element $L$ of the quotient field $\mathbb{K}$ of $\square \widehat{\otimes}_{\mathcal{O}} O_{\Omega}$ can be regarded as a ( $p$-adic) meromorphic function on $\operatorname{Spec}(\mathbb{\square})(\Omega)$ assigning the value $L(P)=P(L)$ to $P \in \operatorname{Spec}(\mathbb{Q})$, where $O_{\Omega}$ is the $p$-adic integer ring of $\Omega$. We call such functions "meromorphic", because it is a ratio of elements in $\square \widehat{\otimes}_{O} O_{\Omega}$ and hence a ratio of $p$-adic analytic functions on a nonempty Zariski open subset of $\operatorname{Spec}(\mathbb{Q})$ (or more precisely, on the formal completion of a non-empty open subscheme of $\operatorname{Spec}(\mathbb{\square})$ along its fibre over $p$ ). Since I is a normal integral domain, if a meromorphic function in the above sense is everywhere defined, it is actually an element in $\square \widehat{\otimes}_{\mathcal{O}} \mathrm{O}_{\Omega}$ (and hence is an Iwasawa function when $\square$ is an irreducible component of $O\left[\left[T\left(\mathbb{Z}_{p}\right)\right]\right]$ for a quotient torus $T$ of $\left.T_{n}\right)$. We expect that

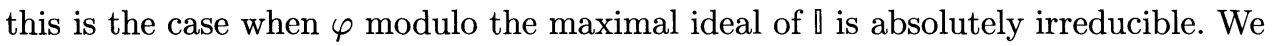
fix a choice of $S=\left\{V_{\mathfrak{B}}^{+}\right\}_{\mathfrak{B}}$. If there exists an element $L_{S, i_{\infty}}(\varphi) \in \mathbb{K}$ satisfying the following condition for all motivic points $P$ as in (A1-2_):

$$
i_{p}^{-1}\left(\frac{L_{S, i_{\infty}}(P, \varphi)}{c_{p, i_{\infty}}^{+}\left(M_{P}(1)\right)}\right)=* i_{\infty}^{-1}\left(\frac{L_{i_{\infty}}\left(1, M_{P}\right)}{c_{\infty, i_{\infty}}^{+}\left(M_{P}(1)\right)}\right)
$$

for a constant “*”, we call $L_{S, i_{\infty}}(\varphi)$ a genuine $p$-adic $L$-function of $\varphi$ of type $S$. The exact form of the constant "*" is known by Coates, Perrin-Riou and Panchishkin, when $M_{P}$ is crystalline at $p$ and $\varphi$ contains all cyclotomic deformation of $M_{P}$ (see, [60], [56] Section 6, and Conjecture 4.2.1 in Chapter 4 in the text). Here $c_{p, i_{\infty}}^{+}\left(M_{P}(1)\right)$ 
is the $p$-adic period defined in exactly the same way as $c_{\infty, i_{\infty}}^{+}\left(M_{P}(1)\right)$ replacing $\mathbb{C}$ by the Fontaine's graded ring $B_{H T}$ (cf. [60]). We note that the period $c_{p, i_{\infty}}^{+}\left(M_{P}(1)\right)$ actually depends on the choice of $i_{\infty}$ besides its apparent dependence on $i_{p}$ and on $S$. From definition, the $p$-adic period depends on the choice of $S$, but we do not know how its transcendency depends on $S$, because there might be some non-trivial relation among periods indexed by $p$-adic places. On the other hand, the complex $L$ value applied $i_{\infty}^{-1}$ at the right-hand side of (Int) is independent of $i_{\infty}$, because, if we change $i_{\infty}$ by $\rho i_{\infty}$ for $\rho \in A u t(\mathbb{C})$, the $L$-value of the right-hand side is conjectured by Deligne to be moved by $\rho$ whose effect will be canceled by $\left(\rho i_{\infty}\right)^{-1}$. We will show in Chapter 2 from the admissibility $\left(\mathrm{A} 2_{+}\right)$(ii) that $c_{p, i_{\infty}}^{+}\left(M_{P}(1)\right)$ lies in a single graded component $\Omega t^{d}$ of $B_{H T}$ and hence can be identified with an element in the $p$ adic completion $\Omega$ of $\overline{\mathbb{Q}}_{p}$ (by trivialization, i.e., just putting $t=1$ ). By construction, $c_{p, i_{\infty}}^{+}\left(M_{P}(1)\right)$ and $c_{\infty, i_{\infty}}^{+}\left(M_{P}(1)\right)$ behave in exactly the same way under the change of basis. That is, by abusing notation, $c_{p, i_{\infty}}^{+}\left(M_{P}(1)\right) / c_{\infty, i_{\infty}}^{+}\left(M_{P}(1)\right)$ is constant, and the formula (Int) has an intrinsic meaning even if these periods depend on the choice of the basis of various realization of motives. This type of definition of $p$-adic periods has been given in various context (see [3] and [74]), but here emphasis is put on its dependence on $i_{\infty}, i_{p}$ and $S$. This type of formulation of $p$-adic $L$-functions is first found by Katz in his construction of $p$-adic Hecke $L$-functions [52] and in this general form, it is due to Don Blasius as indicated in [56] Conjecture II' $^{\prime}$ in Appendix. We study this type of $L$-functions and list some general conjectures in Chapter 4 .

So far, it looks that things are going well. However there is a subtle fact (pointed out to me by Don Blasius) that we do not know whether $c_{p, i_{\infty}}^{+}\left(M_{P}(1)\right) \neq 0$ or not, and André [1] has given fairly general examples of the vanishing for some special choice of $i_{\infty}$ (see 3.3 in the text). We expect to be able to go around this difficulty by changing $i_{\infty}$. In [1], the transcendence of the field spanned by $p$-adic periods moving around $i_{p}$ is studied, and some estimate of its transcendental degree is given for generic $i_{p}$. The embedding $i_{p}$ is intrinsically determined by $\varphi$ (at least on $E_{\infty}$ ), and changing $\left(i_{\infty}, i_{p}\right)$ to $\left(i_{\infty} \sigma, i_{p} \sigma\right)$ for $\sigma \in \mathscr{G}_{\mathbb{Q}}$ does not affect anything. Thus changing $i_{\infty}$ is basically the same as changing $i_{p}$. Here we study the dependence of the period relative to $L$-values. As already remarked, the right-hand side of (Int) is independent of $i_{\infty}$ and therefore, it is all right to change $i_{\infty}$. We will show that if one can choose an embedding $i_{\infty}$ so that $c_{p, i_{\infty}}^{+}\left(M_{P}(1)\right) \neq 0$ for one $P$, then $c_{p, i_{\infty}}^{+}\left(M_{P}(1)\right) \neq 0$ for densely populated $P$ satisfying (A2-) for a fixed $S$ (Proposition 4.1.1 in Chapter 4). Moreover we show that if the genuine $p$-adic $L$-function exists for one $i_{\infty}$, it exists for all $i_{\infty}$ with non-vanishing $p$-adic periods. Further, if $p$ is odd and $\varphi$ is self-dual up to characters, there is a canonical way of normalizing $L_{S, i_{\infty}}(\varphi)$ so that the normalized $L_{S}(\varphi)$ depends only on the isomorphism class of $\varphi$ over 》 but not on $i_{\infty}$ (Section 4.3 in Chapter 4). Anyway, the period $c_{p, i_{\infty}}^{+}\left(M_{P}(1)\right)$ detects a "good" choice of 
$i_{\infty}$ making (Int) possible. Thus assuming the existence of $i_{\infty}$ with non-vanishing $c_{p, i_{\infty}}^{+}\left(M_{P}(1)\right)$, it would be reasonable to conjecture the existence of $L_{S, i_{\infty}}(\varphi)$ for such choice of $i_{\infty}$ (Conjecture 4.2.1 in Chapter 4). Once we can specify the constant "*", the uniqueness of $L_{S, i_{\infty}}(\varphi)$ follows from the density requirement in $\left(\mathrm{A} 2_{ \pm}\right)$of motivic points. A way of specifying " $*$ " is known for crystalline $M_{P}$ by Coates, Perrin-Riou and Panchishkin if $\varphi$ contains all cyclotomic deformation (see [60], [58], [56] and Conjecture 4.2.1 in the text); in other words, $\varphi_{P} \otimes \mathcal{N}^{m}$ appears as $\varphi_{Q}$ for some arithmetic point $Q$ for all $m$ with $\mathcal{N}^{m} \equiv 1 \bmod \mathbf{p}(\mathbf{p}=4$ or $p$ according as $p=2$ or not). Here we write $\mathcal{N}$ for the cyclotomic character of $\varphi_{F}$ with $\mathcal{N}\left(\phi_{\mathfrak{l}}\right)=\mathcal{N}(\mathfrak{l})$ for the geometric Frobenius element $\phi_{\mathfrak{r}}$.

We will show the existence of "good" $i_{\infty}$ for every 2-dimensional absolutely irreducible arithmetic representation $\varphi$ and for tensor products obtained from such 2-dimensional Galois representations (Corollary 3.4.2, Theorems 5.2.1 and 5.3.1 in Chapter 5). This process shows that the type $S$ actually governs the form of complex and $p$-adic periods as a product of periods indexed by archimedean places of $F$ as conjectured by Shimura [66], [67] in the complex case (see Chapter 5). In general, the existence of "good" $i_{\infty}$ follows from the Tate-type conjecture asserting that the isomorphism class of a semi-simple regular motive is determined by its Galois representation (Theorem 3.4.1 in Chapter 3).

In this monograph, we have focused our study on the automorphic aspect of $p$-adic $L$-functions. However, they could have an arithmetic aspect, as typically represented by the Iwasawa theory. A precise (but conjectural) construction of analytic and arithmetic $p$-adic $L$-functions of the family of Tate twists of a given crystalline motive is studied in details by B. Perrin-Riou in [58]. Her formulation is different from ours using $B_{\text {cris }}$ (in place of $B_{H T}$ or $\Omega$ here). In a setting more close to ours, Greenberg [28] and [29] describes a conjectural theory of ( $p$-ordinary) Selmer groups associated to arithmetic Galois deformations. In the automorphic theory, the genuine $p$-adic $L$-function is associated to the isomorphism class of $\varphi$ over the field of fractions of $\llbracket$. On the other hand, as Greenberg pointed me out, in the arithmetic theory, the Selmer group is more delicate and depends on the isomorphism class over 『. Although we expect the normalized $L$-function $L_{S}(\varphi)$ to play an important role in the Iwasawa theory for the contragredient $\check{\varphi}$ of $\varphi$, to formulate a main conjecture in our setting, some more work would be necessary to compare our $p$-adic $L$-functions and the arithmetic ones discussed in [58] and [28] (see Sections 4.3-4.7 in Chapter 4).

We define the arithmetic dual $\varphi^{*}$ of $\varphi$ by the contragredient of $\varphi$ tensored by $\mathcal{N}$. If the (normalized) $p$-adic $L$-function $L_{S}(\varphi)$ exists, a principle of locating singularity (coming from the conjectures of Artin and Tate) would be

$\left(\mathrm{AT}_{p}\right)$ For each prime divisor $P$ of $\operatorname{Spec}\left(\bar{\nabla} \mathrm{O}_{\Omega}\right)$, if the normalized $L_{S}(\varphi)$ has a pole at $P$, then $\varphi_{P} \oplus \varphi_{P}^{*}$ contains the trivial representation as a subquotient. 
In some good cases including the case where the residual representation $\varphi \bmod$ $\mathfrak{m}_{\mathbb{}}$ is absolutely irreducible, we expect $\left(\mathrm{AT}_{p}\right)$ to hold, and we find some other (residually reducible) cases where $\left(\mathrm{AT}_{p}\right)$ might not hold, indicating necessity of further modification of $L_{S}(\varphi)$ (see Section 4.7 in Chapter 4 and Section 8.5 in Chapter 8). Thus the problem is quite subtle. In the first draft of this paper, the above principle was stated in a rough form which did not involve $\varphi^{*}$. The importance of counting also $\varphi^{*}$ in addition to $\varphi$ is suggested to the author by R. Greenberg. As already indicated above, the arithmetic side of the theory of $p$-adic $L$-functions of deformations of motives is discussed in [28] in terms of their Selmer groups. From his point of view ([28] Conjecture 4.1),

(G) the order of the pole should be the exponent $\ell_{P}$ of $P$ in the characteristic ideal of the $\square$-module $H_{0}\left(\mathscr{G}_{F}, \varphi \oplus \varphi^{*}\right)$.

In [28], the conjecture is stated in terms of $H^{0}\left(\varphi_{F}, V\left(\varphi \oplus \varphi^{*}\right)^{\vee} \otimes_{\mathbb{1}} \rrbracket^{*}\right)$, where $\mathbb{0}^{*}$ is the Pontryagin dual module of $\llbracket$. This cohomology group is the Pontryagin dual of $H_{0}\left(\mathscr{G}_{F}, \varphi \oplus \varphi^{*}\right)$. This is the reason why we have Galois homology group in place of cohomology group. Because of this, in principle, the genuine $p$-adic $L$-function should be closely related to the characteristic ideal of the $\operatorname{Selmer} \operatorname{group} \operatorname{Sel}(\check{\varphi})$ (defined in [28]) of the contragredient $\check{\varphi}$ of $\varphi$, although it might be a bit premature to make a main conjecture in this context. Hence $\left(\mathrm{AT}_{p}\right)$ is an assertion similar to (but weaker than) Conjecture 4.1 in [28], which is verified in various examples in [28]. In Chapters 7 and 8, we study the Katz $p$-adic $L$-functions interpolating Hecke $L$-values of CM fields, see that the $L$-function is close to be genuine and establish a stronger version of $\left(\mathrm{AT}_{p}\right)$. Actually Colmez made a precise conjecture in this case [13], and this type of fact has been shown by him [12] and Schneps [14] for abelian $p$-adic $L$-functions for either totally real $F$ or a number field $F$ containing an imaginary quadratic field. I should mention that our proof in the CM case is just a reinterpretation of the result of Colmez for totally real $F$.

When $F$ is totally real, out of Hilbert modular forms on $G L(2)_{/ F}$, we can construct examples of arithmetic Galois representations for $n=2$. Let $\mathbf{h}$ be the universal ordinary or nearly ordinary $p$-adic Hecke algebra studied in [34], [35] and [33] Chapter 7 (the theory is recalled in Chapter 2). Then we have a canonical representation $\pi$ of $\mathscr{G}_{F}$ taking values roughly in $G L_{2}(\mathbf{h})$ [38] (see Section 2.8 in

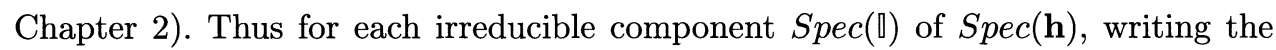

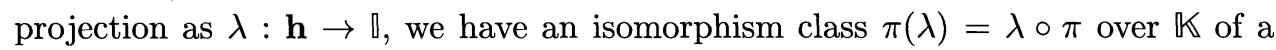
Galois representation. We can describe the relation between $\pi(\lambda)$ and the Galois representations of classical Hilbert modular forms ([9], [69], [6]) in the following way. The Hecke algebra is naturally an algebra over the completed group algebra $\mathcal{O}\left[\left[T_{2}\left(\mathbb{Z}_{p}\right)\right]\right]$. By (A3) the algebra structure factors through a quotient torus $T$ of $T_{2}$. We call $T$ the weight group and describe it in details in Section 2.1 in 
Chapter 2. We call an arithmetic point $P$ positive if it induces locally on $T\left(\mathbb{Z}_{p}\right)$ a positive weight $\kappa(P)$ of $T_{2}$ with respect to the upper-triangular Borel subgroup of $G L(2)$. If $P$ is positive arithmetic, the reduction $\pi\left(\lambda_{P}\right)=P \circ \pi(\lambda)$ modulo $P$ is the Galois representation of a classical Hilbert modular form of weight $\kappa(P)$. As shown in [6], to such $\pi\left(\lambda_{P}\right)$, we have a rank two motive $M\left(\lambda_{P}\right)$ such that $L\left(s, M\left(\lambda_{P}\right)\right)=L\left(s, \pi\left(\lambda_{P}\right)\right)$; thus, positive arithmetic points are motivic. Therefore, $\varphi$ in the class $\pi(\lambda)$ (over $\mathbb{K}$ ) is arithmetic, and so are their tensor products. There are several choices of $V_{\mathfrak{B}}^{+}=V_{\mathfrak{B}}^{-}$in the case of $\pi\left(\lambda_{1}\right) \otimes \ldots \otimes \pi\left(\lambda_{n}\right)(n>1)$ for a given choice for each $\pi\left(\lambda_{i}\right)$. Each choice $S=\left\{V_{\mathfrak{B}}^{+}\right\}_{\mathfrak{B} \mid p}$ should yield a $p$-adic $L$-function satisfying (Int) for motivic points $P$ compatible with the choice $S$. We make this precise in Conjectures 5.4.1 and 5.5.1 in Chapter 5. In the abelian (or rather CM) case, the choice of $S$ gives rise to a $p$-adic CM type to which we attach a Katz $p$-adic $L$-function ([52] and [47]) and, even in the non-abelian case, is very much related to factorization of motivic periods into product of local periods indexed by archimedean places as discussed in [66], [67], [3], [5], [30], [31], [74], [75] and [42].

Various $p$-adic $L$-functions supported on the spectrum $\operatorname{Spec}(\mathbf{h})$ or $\operatorname{Spec}\left(\mathbf{h} \widehat{\otimes}_{\mathbb{O}} \mathbf{h}\right)$ are studied in the works [47], [33], [37], [39], [40], [41], [53] and [32]. These $p$-adic $L$-functions include the $p$-adic Rankin product $D_{p}\left(\pi(\lambda) \otimes \pi(\mu)^{\vee}\right) \in \mathbb{K} \widehat{\otimes}_{\mathfrak{O}} \mathbb{V}$ of $\pi(\lambda)$ and $\pi(\mu)$ for $\lambda: \mathbf{h} \rightarrow \llbracket$ and $\mu: \mathbf{h} \rightarrow \mathbb{J}$, where $\pi(\mu)^{\vee}$ is the contragredient of $\pi(\mu)$. In Chapter 6 , we study $D_{p}$ through the conjectural theory described above. Although we can easily identify the class $S=\left\{V_{\mathfrak{B}}^{+}\right\}_{\mathfrak{B} \mid p}$ to which $D_{p}$ belongs, there are several reasons to believe that $D_{p}$ is not a single genuine $p$-adic $L$-function but a ratio of two genuine $L$-functions. To explain this, we pick two representations $\varphi$ and $\rho$ in the class $\pi(\lambda)$ and $\pi(\mu)$. The isomorphism class of $\varphi$ over " may not be uniquely determined by $\pi(\lambda)$. It is known by Carayol [10] that $\pi(\lambda)$ determines uniquely $\varphi$ if (AI) $\bar{\varphi}=\varphi \bmod \mathfrak{m}$ for the maximal ideal $\mathfrak{m}$ of $\llbracket$ is absolutely irreducible.

The most significant reason among them is that $D_{p}\left(\pi(\lambda) \otimes \pi(\mu)^{\vee}\right)$ has poles at prime divisors in the support of the congruence module $C_{0}(\lambda ; \square)$ (see Section 2.9 in Chapter 2). Since $\varphi_{P} \otimes \rho_{Q}^{\vee}$ has the trivial representation as a subquotient if $\varphi_{P} \cong \rho_{Q}$, it looks all right to have a pole at the intersection of the two irreducible components $\lambda$ and $\mu$, since the support $X$ of $C_{0}(\lambda ; \square)$ is the locus of $P$ such that $\lambda_{P}=\mu_{P}^{\prime}$ for irreducible components $\mu^{\prime}$ other than $\lambda$ (including $\mu$ ). This is not correct because the two variable $L$-function: $(P, Q) \mapsto D_{p}\left(P, Q ; \pi(\lambda) \otimes \pi(\mu)^{\vee}\right)$ has a pole on $X \times \operatorname{Spec}(\mathbb{U})$ concentrated on the variable $P$, which is nothing to do with the locus $W$ of $(P, Q)$ satisfying $\varphi_{P} \cong \rho_{Q}$. Moreover it is easy to show that $W$ is contained in the union of the diagonal $\Delta$ and the diagonal image $W^{\prime}$ of $X$ in $\operatorname{Spec}\left({ } \widehat{\otimes}_{\bigcirc} \downarrow\right)$. The latter $W^{\prime}$ is of codimension at least 2 by definition, and the diagonal $\Delta$ is at least codimension 2 in the nearly ordinary case. Therefore if $D_{p}\left(\pi(\lambda) \otimes \pi(\mu)^{\vee}\right)$ were genuine, it would have to be holomorphic at $W^{\prime}$ and $X \times \operatorname{Spec}(\mathbb{\nabla})$. In [47], when $\lambda$ and $\mu$ are both of 
CM-type for the same CM field, we computed $D_{p}\left(\pi(\lambda) \otimes \pi(\mu)^{\vee}\right)$ in terms of $p$-adic Hecke $L$-functions of Katz and found that it is basically equal to the ratio

$$
D_{p}\left(P, Q ; \pi(\lambda) \otimes \pi(\mu)^{\vee}\right)=\frac{L_{S, i_{\infty}}(P \otimes Q ; \varphi \otimes \check{\rho})}{L_{p, i_{\infty}}(P, A d(\varphi))},
$$

where $L_{S, i_{\infty}}(\varphi \otimes \check{\rho})$ (resp. $\left.L_{p, i_{\infty}}(A d(\varphi))\right)$ is the "genuine" $p$-adic $L$-functions for the

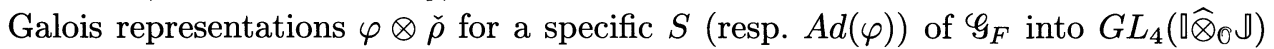

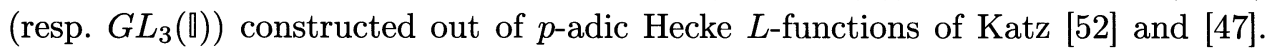

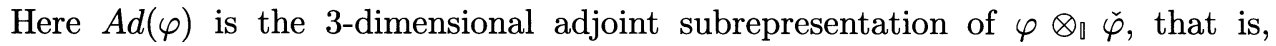
$\varphi \otimes_{\mathbb{1}} \check{\varphi}=A d(\varphi) \oplus \mathbf{1}$ for the trivial representation $\mathbf{1}$. We wrote "genuine" for the above $L$-functions because it is not yet completely proven that the $L$-functions are genuine, strictly speaking (see Chapters 7 and 8). For the proof of genuineness, we need to suppose the compatibility of two comparison maps: one is that of Tate-Raynaud and the other is due to Faltings (see [50] 4.3.5). This fact is also pointed out to me by Blasius. On the other hand, if it is genuine, it is automatically normalized. Anyway this suggests us the same scenario in general. In fact, (i) the ratio (R) only depends on $\pi(\lambda) \otimes \pi(\mu)^{\vee}$ but not on $i_{\infty}$ (see (NP) in Section 4.3 and Theorem 5.2.1 in Chapter 4), (ii) the formula (R) is a consequence of the existence of two genuine $p$-adic $L$-functions $L_{S, i_{\infty}}(\varphi \otimes \check{\rho})$ and $L_{p, i_{\infty}}(A d(\varphi))$, and (iii) only assuming (AI), $p \geq 5$ and the existence of the normalized $L_{p}(A d(\varphi))$ in $I \widehat{\otimes}_{\mathcal{O}} \Omega$, we can show that the normalized function $L_{S}(\varphi \otimes \check{\rho})$ defined by $L_{p}(A d(\varphi)) D_{p}\left(\pi(\lambda) \otimes \pi(\mu)^{\vee}\right)$ is everywhere holomorphic if one admits the following

Conjecture 1.0.1 - Under (AI) and $p \geq 5$, we have

$$
C_{0}(\lambda ; \rrbracket) \otimes_{\mathcal{O}} 0_{\Omega} \cong \square \widehat{\otimes}_{\mathcal{O}} 0_{\Omega} /\left(L_{p}(A d(\varphi))\right)
$$

as $\square \widehat{\otimes}_{\odot} 0_{\Omega}-$ modules.

This is a generalization of a conjecture made in [47]. We know that the conjecture holds for CM components when $F=\mathbb{Q}$ by the works [55], [71] and [47] combined, and we have everything we want when $F=\mathbb{Q}$ and $\lambda$ is of CM-type (but $\mu$ can be non-abelian; see Chapter 7). Since the result of [41] supplies an evidence of the conjectures, I added at the end of this paper a list of corrections of misstatements in [41] to avoid confusion. Some other evidences supporting the conjecture are also discussed in [19]. We will use throughout the paper the notation introduced in the introduction. I would like to thank Don Blasius and R. Greenberg for their pointing me out many subtle points in the theory of motives and Selmer groups as indicated above.

The first draft of this monograph was prepared while the author was visiting the Mathematical Science Research Institute at Berkeley in 1995. The visit was supported by the grant from NSF: DMS 9022140. The author also likes to 
acknowledge the partial support he obtained from NSF (DMS 9401026) while preparing the final version. 



\section{2. $p$-Adic Hecke algebras}

We shall give a summary of the theory of $p$-adic Hecke algebras developed in [34], [35], [36], [41] and [47]. The formulation is a bit different from earlier treatment reflecting evolution. We assume $F$ to be totally real in this chapter.

\subsection{The weight group}

First we like to specify the notion of weight for Hilbert modular forms for $F$. Let $T_{1}=\operatorname{Res}_{\mathfrak{r} / \mathbb{Z}} \mathbf{G}_{m}$, where $\mathfrak{r}$ is the integer ring of $F$. Then $T_{1}$ is an algebraic torus over $\mathbb{Z}$ such that $T_{1}(A)=\left(\mathfrak{r} \otimes_{\mathbb{Z}} A\right)^{\times}$. We identify the character group $X\left(T_{1}\right)$ of $T_{1}$ with $\mathbb{Z}[I]$ so that $n=\sum_{\sigma \in I} n_{\sigma} \sigma$ sends $x \in T_{1}(\mathbb{Q})=F^{\times}$to $x^{n}=\prod_{\sigma \in I} x^{\sigma n_{\sigma}}$ in $\overline{\mathbb{Q}}^{\times}$. Weights are well described by identifying $T_{1} \times_{\mathbb{Z}} T_{1}$ with the standard maximal $F$-split torus of the algebraic group $G=\operatorname{Res}_{\mathfrak{r} / \mathbb{Z}} G L(2)$, that is, the subgroup of diagonal matrices. Here we specify the identification of $T_{2}$ with $T_{1} \times T_{1}$, reversing the order of diagonal entries, by $T_{2} \ni\left(\begin{array}{ll}a & 0 \\ 0 & d\end{array}\right) \leftrightarrow(d, a) \in T_{1} \times T_{1}$. The character group $X\left(T_{1} \times T_{1}\right)$ is given by $\mathbb{Z}[I] \times \mathbb{Z}[I]$. We specify this isomorphism in a standard way so that $(x, y)^{(m, n)}=x^{-m} y^{-n}$. We take the connected component $T_{0}$ of the Zariskiclosure of the diagonal image of $\mathfrak{r}^{\times}$in $T_{2}=T_{1} \times T_{1}$, which is again a torus. For $T=\left(T_{1} \times T_{1}\right) / T_{0}$, we have

$$
X(T)=\left\{(m, n) \in X\left(T_{1} \times T_{1}\right) \mid m+n \in \mathbb{Z} t\right\}
$$

for $t=\sum_{\sigma} \sigma$. We now enlarge a bit the group $T\left(\mathbb{Z}_{p}\right)$. Fix an open compact subgroup $U$ of $G\left(\mathbb{A}^{(\infty)}\right)$ with $U \supset G L_{2}\left(\mathfrak{r}_{p}\right)$, and write $E_{U}$ for $U G_{+}(\mathbb{R}) \cap F^{\times}$for the connected component of the identity $G_{+}(\mathbb{R})$ of $G(\mathbb{R})$, where $\mathbb{A}^{(\infty)}$ is the ring of finite adeles. Then we put

$$
\mathbf{G}=\mathbf{G}_{U}=\left(T_{1} \times T_{1}\right)\left(\mathbb{Z}_{p}\right) / \bar{E}_{U},
$$

where $\bar{E}_{U}$ is the closure of $E_{U}$ under the $p$-adic topology. A typical example of $U$ is given by

$$
\widehat{\Gamma}_{1}(N)=\left\{\left(\begin{array}{ll}
a & b \\
c & d
\end{array}\right) \in G(\widehat{\mathbb{Z}}) \mid c \equiv d-1 \equiv 0 \quad \bmod N \widehat{\mathfrak{x}}\right\} \quad\left(\widehat{\mathbb{Z}}=\prod_{\ell} \mathbb{Z}_{\ell}, \quad \widehat{\mathfrak{r}}=\prod_{\mathfrak{l}} \mathfrak{r}_{\mathfrak{l}}\right)
$$


for an ideal $N$ prime to $p$. We use the $p$-adic character group $\mathscr{X}=\operatorname{Hom}_{\text {conti }}\left(\mathbf{G}, \Omega^{\times}\right)$ of $\mathbf{G}$ as the parameter space of $p$-adic nearly ordinary families of Hilbert modular forms. Although the full nearly ordinary family is parameterized by the space $\mathscr{X}$, the smaller ordinary family is parameterized by the closed subspace of $\mathscr{X}^{\text {ord }}$ in $\mathscr{X}$ which is made of characters factoring through

$$
\mathbf{G}^{\text {ord }}=\mathbf{G}_{U}^{\text {ord }}=\mathbf{G} /\left(\{1\} \times T_{1}\left(\mathbb{Z}_{p}\right)\right) \cong T_{1}\left(\mathbb{Z}_{p}\right) / \bar{E}_{U} .
$$

The subgroup $X\left(T_{1} \times T_{1}\right)$ made of characters trivial on $E_{U}$ is a subgroup of finite index in $X(T)$. We fix an embedding $i_{p}: \overline{\mathbb{Q}} \hookrightarrow \Omega$. Then each $m \in X(T)=$ $\operatorname{Hom}_{A l g-g r}\left(T_{\overline{\mathbb{Q}}}, \mathbf{G}_{m / \overline{\mathbb{Q}}}\right)$ induces $m \circ i_{p}$ in $\operatorname{Hom}_{g r}\left(T(\Omega), \Omega^{\times}\right)$. A continuous character $\kappa$ in $\mathscr{X}$ is called an arithmetic weight (or character) if on a $p$-adic neighborhood of the identity, $\kappa$ coincides with an algebraic character in $X(T)$ via $i_{p}$. This notion of being arithmetic is independent of the choice of $i_{p}$. We write $\mathscr{A}$ for the subgroup of arithmetic weights in $\mathscr{X}$. We write additively the group $\mathscr{A}$, and thus " 0 " is the identity character of $\mathscr{A}$. We put $\mathscr{A}^{\text {ord }}=\mathscr{A} \cap \mathscr{X}^{\text {ord }}$. We write $(m(\kappa), n(\kappa)) \in X(T)$ for the algebraic character induced by an arithmetic weight $\kappa$. An arithmetic weight $\kappa$ is called positive if $m(\kappa)-n(\kappa)>0$ (that is, $m(\kappa)_{\sigma}>n(\kappa)_{\sigma}$ for all $\sigma$ ). Then

$$
\varepsilon_{\kappa}(a, d)=\kappa(a, d)(d, a)^{-(m(\kappa), n(\kappa))}
$$

is a finite order character of $\mathbf{G}$, and by definition, $\kappa \in \mathscr{A}^{\text {ord }} \Leftrightarrow \kappa \in \mathscr{A}, \varepsilon_{\kappa}$ is trivial on the variable $a$ and $n(\kappa)=0$.

In our previous works [34], [35], [38], [41] (resp. [42]), we have used weight written as $(k, w)$ (resp. $(n, v))$ directly related to the automorphic factors (resp. the cohomological weight) of modular forms, while the weight $(m, n)$ comes from the Hodge type of motives attached to modular forms. The following formulas clarify the relation among the three formulations:

$$
(k, w) \leftrightarrow(m-n+t, t-n) \quad\left(t=\sum_{\sigma \in I} \sigma\right) \quad \text { and } \quad(n, v) \leftrightarrow(m-n-t, n) .
$$

A motivic and deformation theoretic description of the parameter spaces and conjectures predicting its general form can be found in Panchishkin [56] Appendix and in Tilouine [72].

\subsection{Automorphic forms of weight $\kappa$}

We choose an embedding $\iota: \overline{\mathbb{Q}} \hookrightarrow \mathbb{C}$. Then we may regard $m \in X(T)=$ $\operatorname{Hom}_{A l g-g r}\left(T_{/ \overline{\mathbb{Q}}}, \mathbf{G}_{m / \overline{\mathbb{Q}}}\right)$ as an element $m \iota$ in $\operatorname{Hom}_{g r}\left(T(\mathbb{C}), \mathbb{C}^{\times}\right)$. Then we consider the following automorphic factor for each $\kappa \in \mathscr{A}$ :

$$
J_{\kappa \iota}(g, z)=\operatorname{det}(g)^{n(\kappa) \iota-t \iota}(c z+d)^{m(\kappa) \iota-n(\kappa) \iota+t \iota}=\operatorname{det}(g)^{-w \iota}(c z+d)^{k \iota},
$$


where $z \in T_{1}(\mathbb{C})=\left(F \otimes_{\mathbb{Q}} \mathbb{C}\right)^{\times}$and $g=\left(\begin{array}{ll}a & b \\ c & d\end{array}\right) \in G(\mathbb{R})$. We assume that $\varepsilon_{\kappa}$ factors through $\left(T_{1} \times T_{1}\right)\left(\mathbb{Z} / p^{\alpha} \mathbb{Z}\right)$ and put

$$
\begin{aligned}
& U_{0}\left(p^{\alpha}\right)=\left\{u \in U \mid u_{p}=\left(\begin{array}{ll}
a & b \\
c & d
\end{array}\right) \text { and } c \in p^{\alpha} \mathfrak{r}_{p}\right\} \text { and } \\
& U_{1}\left(p^{\alpha}\right)=\left\{u \in U_{0}\left(p^{\alpha}\right) \mid u_{p}=\left(\begin{array}{ll}
a & b \\
c & d
\end{array}\right) \text { and } d \equiv 1 \quad \bmod p^{\alpha} \mathfrak{r}_{p}\right\}, \\
& U_{1}^{1}\left(p^{\alpha}\right)=\left\{u \in U_{1}\left(p^{\alpha}\right) \mid u_{p}=\left(\begin{array}{ll}
a & b \\
c & d
\end{array}\right) \text { and } a \equiv 1 \quad \bmod p^{\alpha} \mathfrak{r}_{p}\right\},
\end{aligned}
$$

where $\mathfrak{r}_{p}=\mathfrak{r} \otimes_{\mathbb{Z}} \mathbb{Z}_{p}$. Let $G_{+}(\mathbb{R})$ be the identity connected component of the Lie group $G(\mathbb{R})$. We write $C_{\infty}$ for the standard maximal compact subgroup of $G(\mathbb{R})$, and we put $8=G(\mathbb{R}) / C_{\infty} Z(\mathbb{R})=G_{+}(\mathbb{R}) / C_{\infty+} Z(\mathbb{R})$ for the center $Z$ of $G$ and $C_{\infty+}=C_{\infty} \cap G_{+}(\mathbb{R})$. Then we may identify 3 with $\mathfrak{S}^{I}$ for the upper half complex plane $\mathfrak{S}$ so that $G_{+}(\mathbb{R}) \ni\left(\begin{array}{ll}y & x \\ 0 & 1\end{array}\right) \mapsto x+\mathbf{i} y \in \mathfrak{S}^{I}$, where we write the $\mathbf{i}$ for $(\sqrt{-1}, \ldots, \sqrt{-1})$ in 8 . We write $\varepsilon_{\kappa, \iota}$ for $\iota \circ i_{p}^{-1} \circ \varepsilon_{\kappa}$. A modular form $f: G(\mathbb{A}) \rightarrow \mathbb{C}$ of weight $\kappa$ with respect to an open subgroup $V$ of $U_{0}\left(p^{\alpha}\right)$ satisfies the following automorphic condition:

$$
f(a x u)=\varepsilon_{\kappa, \iota}(a, d) f(x) J_{\kappa \iota}\left(u_{\infty}, \mathbf{i}\right)^{-1} \quad \text { for } \quad u \in V C_{\infty+} Z(\mathbb{R}) \text { and } a \in G(\mathbb{Q}),
$$

where we have written $u_{p}=\left(\begin{array}{ll}a & b \\ c & d\end{array}\right)$. By the above formula, for $z \in Z(\mathbb{R})\left(\cong T_{1}(\mathbb{R})\right)$, we see $f(z x)=z^{-m-n+t} f(x)(m=m(\kappa)$ and $n=n(\kappa))$. Thus if $f$ has the central character (that is, $f(x z)=\chi(z) f(x)$ for $z \in Z(\mathbb{A})$ ), its infinity type is given by $-w(\kappa)+t$ for $w(\kappa)=m(\kappa)+n(\kappa)$. Anyway, we define $\left.f|\langle z\rangle(x)=| z\right|_{\mathbb{A}} ^{-1} f(x z)$ for $z \in Z\left(\mathbb{A}^{(\infty)}\right)$. In particular, for a prime ideal $\mathfrak{l}$ such that $G L_{2}\left(\mathfrak{x}_{\mathfrak{l}}\right) \subset V$, we write $\langle\mathfrak{l}\rangle$ for the operator $\langle\varpi\rangle$, where $\varpi$ is a prime element of $\mathfrak{r}_{\mathfrak{l}}$. The operator $\langle\mathfrak{l}\rangle$ is well defined independent of the choice of $\varpi$. If $\mathfrak{a}=\prod_{\mathfrak{l}} \mathfrak{l}^{(\mathfrak{l})}$ is a product of primes $\mathfrak{l}$, we define $\langle\mathfrak{a}\rangle=\prod_{\mathfrak{r}}\langle\mathfrak{l}\rangle^{e(\mathfrak{l})}$.

Now we interpret the condition $(\mathrm{S} 1)$ in terms of the classical automorphy condition. Define a function $f_{x}$ on 3 for each $x \in G\left(\mathbb{A}^{(\infty)}\right)$ by $f_{x}(z)=$ $f\left(x u_{\infty}\right) J_{\kappa}\left(u_{\infty}, \mathbf{i}\right)$ for $u_{\infty} \in G_{+}(\mathbb{R})$ with $u_{\infty}(\mathbf{i})=z$. This function is determined independently of the choice of $u_{\infty}$ by (S1), and it is straightforward to check that

$$
f_{x}(\gamma(z))=f_{x}(z) J_{\kappa}(\gamma, z) \text { for } \gamma \in \Gamma_{x}=x V G_{+}(\mathbb{R}) x^{-1} \cap G(\mathbb{Q}) .
$$

Then we further impose

(S2) $f_{x}(z)$ is a holomorphic function on 8 for all $x \in G\left(\mathbb{A}^{(\infty)}\right)$ and is rapidly decreasing at all cusps of $G$.

When we consider non-cuspidal modular forms, we instead impose

(M2) $f_{x}(z)$ is a holomorphic function on 3 for all $x \in G\left(\mathbb{A}^{(\infty)}\right)$ and is slowly increasing at all cusps of $G$.

It is well known that the condition (M2) and (S2) are equivalent if $n \notin \mathbb{Z} t$ for $t=\sum_{\sigma} \sigma$. 


\subsection{Fourier-expansion and rationality}

We choose a finite idele $d$ such that $d \mathfrak{r}$ is the different of $F / \mathbb{Q}$. Write $F_{\mathbb{A}}=F \otimes_{\mathbb{Q}} A$ and $F_{\mathbb{A}(\infty)}=F \otimes_{\mathbb{Q}} \mathbb{A}^{(\infty)}$. Each modular form $f$ satisfying (S1-2) has the following type of Fourier expansion on $G(\mathbb{A})=G L_{2}\left(F_{\mathbb{A}}\right)$ (see [34] Section 4 and [41] Section 2 for details):

$$
f\left(\left(\begin{array}{ll}
y & x \\
0 & 1
\end{array}\right)\right)=|y|_{\mathbb{A}} \sum_{\xi \gg 0} a(\xi y d, f)\left(\xi y_{\infty}\right)^{-n} \mathbf{e}_{F}\left(\sqrt{-1} \xi y_{\infty}\right) \mathbf{e}_{F}(\xi x),
$$

where $\mathbf{e}_{F}: F_{\mathbb{A}} / F \rightarrow \mathbb{C}^{\times}$is the additive character such that

$$
\mathbf{e}_{F}(x)=\exp \left(2 \pi \sqrt{-1} \sum_{\sigma} x_{\sigma}\right)
$$

for $x=\left(x_{\sigma}\right)_{\sigma \in I} \in \mathbb{C}^{I}=F \otimes_{\mathbb{Q}} \mathbb{C}, \xi$ runs over all totally positive elements in $F$, and $y \mapsto a(y, f)$ is a function on $F_{\mathbb{A}(\infty)}^{\times}$invariant under $\left\{q \in F_{\mathbb{A}(\infty)}^{\times} \mid\left(\begin{array}{ll}q & 0 \\ 0 & 1\end{array}\right) \in V\right\}$. When $U$ contains $\widehat{\Gamma}_{1}(N)$ and $V \supset U_{1}^{1}\left(p^{\alpha}\right)$, the function $y \mapsto a(y, f)$ is supported on integral ideles. Here an integral idele means an element in $F_{\mathbb{A}^{(\infty)}}^{\times} \cap \widehat{\mathfrak{r}}$ for $\widehat{\mathfrak{r}}=\prod_{\mathfrak{r}} \mathfrak{r}_{\mathfrak{r}}$. When we consider non-cuspidal holomorphic modular form, we need to add the "constant term" to the above Fourier expansion. Namely modular form satisfying (M2) and (S1) for $n=[n] t$ with $[n] \in \mathbb{Z}$ has the following Fourier expansion:

$$
f\left(\left(\begin{array}{ll}
y & x \\
0 & 1
\end{array}\right)\right)=|y|_{\mathbb{A}}\left\{a_{0}(y d, f)|y|_{\AA}^{-[n]}+\sum_{\xi \gg 0} a(\xi y d, f)\left(\xi y_{\infty}\right)^{-n} \mathbf{e}_{F}\left(\sqrt{-1} \xi y_{\infty}\right) \mathbf{e}_{F}(\xi x)\right\},
$$

where the constant term $a_{0}$ is a function on $F_{\mathrm{A}(\infty)}^{\times}$factoring through the strict ray class group $C l_{F}(N)$ modulo $N$ for sufficiently small integral ideal $N$.

For any $\mathbb{Q}$-subalgebra $A$ of $\overline{\mathbb{Q}}$, we define $S_{\kappa \iota}(V ; \iota(A))$ to be the space of functions satisfying (S1-2) and whose Fourier coefficients $a(y, f)$ are in $\iota(A)$ for all $y \in F_{\mathrm{A}^{(}(\infty)}^{\times}$. If we change $\iota$ to $\iota \sigma$ for $\sigma \in \mathscr{G}_{\mathbb{Q}}$, then by a result of Shimura (see [65] Section 1 and [34] Theorem 4.4), the natural action induced by $a(y, \iota \sigma(f))=\iota \sigma \iota^{-1}(a(y, \iota(f)))$ (for all $y$ ) takes $S_{\kappa \iota}(V ; \iota(A))$ isomorphically to $S_{\kappa \sigma \iota}(V ; \iota \sigma(A))$. Thus we have an intrinsically defined $A$-module $S_{\kappa}(V ; A)$ (independent of the choice of $\iota$ ) and an intrinsic function $a(y, f)$ having values in $A$ such that $\iota$ induces an isomorphism $\iota: S_{\kappa}(V ; A) \cong S_{\kappa \iota}(V ; \iota(A))$ with $\iota(a(y, f))=a(y, \iota(f))$. When $U$ is given by $\widehat{\Gamma}_{1}(N)$ for an r-ideal $N$ prime to $p$ and $V=U_{0}\left(p^{\alpha}\right)$, we write $S_{\kappa}\left(N p^{\alpha} ; A\right)$ for $S_{\kappa}(V ; A)$. In a similar manner as above, we can define the space $M_{\kappa}(V ; A)$ of holomorphic modular forms (given by (S1) and (M2)) requiring further $a_{0}(y, f)$ to have values in $\iota(A)$.

\section{$2.4 q$-Expansion, integrality and Hecke operators}

Let $K$ be a $p$-adically complete subfield inside the $p$-adic completion $\Omega$ of $\overline{\mathbb{Q}}_{p}$ and $\mathcal{O}$ be the $p$-adic integer ring of $K$. Then we may consider $K_{0}=i_{p}^{-1}\left(i_{p}(\overline{\mathbb{Q}}) \cap K\right)$ 
and $S_{\kappa}(V ; K)=S_{\kappa}\left(V ; K_{0}\right) \otimes_{K_{0}, i_{p}} K$. We may regard via $i_{p}$ Fourier coefficients of a modular form in $S_{\kappa}\left(V ; K_{0}\right)$ as a function on $F_{\mathrm{A}(\infty)}^{\times}$into $K$. In particular, we can extend the function $f \mapsto a(y, f)$ to $S_{\kappa}(V ; K)$ by linearlity. Then for $f \in S_{\kappa}(V ; \Omega)$, we define the $q$-expansion coefficients by

$$
\mathbf{a}_{p}(y, f)=y_{p}^{-n} a(y, f) \text { and } \mathbf{a}_{0, p}(y, f)=\mathcal{N}\left(y d^{-1}\right)^{[n]} a_{0}(y, f),
$$

where $\mathcal{N}: F_{\mathbb{A}^{(\infty)}}^{\times} \rightarrow \mathbb{Z}_{p}^{\times}$is the cyclotomic character such that $\mathcal{N}(y)=y_{p}^{-t}|y|_{\mathbb{A}}^{-1}$ for $y \in F_{\mathrm{A}(\infty)}^{\times}$(cf. ([41] Sections 1-3)). Let $F_{+}$be the additive monoid of totally positive elements of $F$. We now identify $f$ with a function on $F_{A(\infty)}^{\times}$having values in the completed monoid algebra $\Omega\left[\left[q^{\xi}\right]\right]_{\xi \in F_{+}} \cong \Omega\left[\left[F_{+}\right]\right]$by

$$
\begin{aligned}
& f(y)=\mathcal{N}^{-1}(y) \sum_{\xi \in F_{+}} \mathbf{a}_{p}(\xi y d, f) q^{\xi} \text { for cusp forms, and } \\
& f(y)=\mathcal{N}^{-1}(y)\left\{\mathbf{a}_{0, p}(y d, f)+\sum_{\xi \in F_{+}} \mathbf{a}_{p}(\xi y d, f) q^{\xi}\right\} \text { for non-cuspidal forms. }
\end{aligned}
$$

We call this expansion the $q$-expansion of $f$. For any closed subring $A$ of $\Omega$, we write $S_{\kappa}(V ; A)$ (resp. $M_{\kappa}(V ; A)$ ) for the subspace made of cusp forms (resp. general modular forms) $f$ with $\mathbf{a}_{p}(y, f) \in A$ (resp. $\mathbf{a}_{p}(y, f) \in A$ and $\mathbf{a}_{0, p}(y, f) \in A$ ) for all $y$. This definition is compatible with the one previously given when $A$ is a $\mathbb{Q}^{-}$ subalgebra. For each prime ideal $\mathfrak{l}$ of $\mathfrak{r}$, we pick a prime element $\varpi_{\mathfrak{l}}$ in $F_{\mathfrak{l}}$. Then we can define the Hecke operator $\mathbb{T}\left(\varpi_{l}\right)$ by

$$
\mathbf{a}_{p}\left(y, f \mid \mathbb{T}\left(\varpi_{\mathfrak{l}}\right)\right)= \begin{cases}\mathbf{a}_{p}\left(y \varpi_{\mathfrak{l}}, f\right)+\mathbf{a}_{p}\left(y \varpi_{\mathfrak{l}}^{-1}, f \mid\langle\mathfrak{l}\rangle\right) & \text { if } \mathfrak{l} \text { is prime to } N p, \\ \mathbf{a}_{p}\left(y \varpi_{\mathfrak{l}}, f\right) & \text { if } \mathfrak{l} \mid N p .\end{cases}
$$

Then this operator $\mathbb{T}\left(\varpi_{I}\right)$ is well defined on $S_{\kappa}(V ; A)$ as long as $U \supset \widehat{\Gamma}_{1}(N)$ and $U_{0}(p) \supset V \supset U_{1}^{1}\left(p^{\alpha}\right)$. The operator $\mathbb{T}\left(\varpi_{\mathfrak{l}}\right)$ for $\mathfrak{I}$ outside $N p$ only depends on the ideal $\mathfrak{l}$; so, we also write $\mathbb{T}(\mathfrak{l})$ for that. Here we have stated the definition of $\mathbb{T}(y)$ when $y=\varpi_{\mathfrak{l}}$ for a prime $\mathfrak{l}$. However, as is well known (e.g. [41] Section 3, where $\mathbb{T}(y)$ is written as $\mathbf{T}(y))$, we can define $\mathbb{T}(y)$ for all integral finite ideles $y$, and we have

$$
\mathbf{a}_{p}(y, f)=\mathbf{a}_{p}(1, f \mid \mathbb{T}(y))
$$

The group $\mathbf{G}$ naturally acts on $S_{\kappa}(V ; \mathbb{C})$ by $f(x) \mapsto f\left(x\left(\begin{array}{cc}a^{-1} & 0 \\ 0 & d^{-1}\end{array}\right)\right)$. We define an action of $\mathbf{G}$ on $S_{\kappa}(V ; \Omega)$ by

$$
f \mid(a, d)(x)=\kappa(a, d) f\left(x\left(\begin{array}{cc}
a^{-1} & 0 \\
0 & d^{-1}
\end{array}\right)\right)
$$

if $V \supset U_{1}^{1}\left(p^{\alpha}\right)$. This action can be written as $f|(a, d)=f| \mathbb{T}\left(a^{-1} d\right)\left\langle d^{-1}\right\rangle$ and hence preserves $S_{\kappa}(V ; A)$ for any closed subalgebra $A$ in $\Omega$. 


\section{$2.5 p$-adic Hecke algebras}

The Hecke algebra $h_{\kappa}(V ; A)$ is the $A$-subalgebra of the $A$-linear endomorphism algebra of $S_{\kappa}(V ; A)$ generated by $\mathbb{T}(y)$ for all $y$ as above and the action of $\mathbf{G}_{U}$. The nearly ordinary part of these algebras is the maximal quotient (and also the maximal algebra direct summand) in which the image of $\mathbb{T}\left(\varpi_{\mathfrak{p}}\right)$ is a unit for all $\mathfrak{p}$ dividing $p \mathfrak{r}$. We write $h_{\kappa}^{n . o r d}$ for the nearly ordinary part, which is the maximal algebra direct factor of $h_{\kappa}$ on which $\mathbb{T}(p)$ is a unit. We write $e$ for the idempotent of the nearly ordinary part. By definition, we have an algebra structure of these Hecke algebras over the completed group algebra $\mathcal{O}[[\mathbf{G}]]$ induced by the action of $\mathbf{G}$. To make explicit the structure of $\mathcal{O}[[\mathbf{G}]]$ and $\mathcal{O}\left[\left[\mathbf{G}^{\text {ord }}\right]\right]$, we write $\mathbf{W}$ (resp. $\mathbf{W}^{\text {ord }}$ ) for the torsion-free part of $\mathbf{G}$ (resp. $\mathbf{G}^{\text {ord }}$ ). Thus

$$
\mathbf{G}=\mathbf{W} \times \mu \quad \text { and } \quad \mathbf{G}^{\text {ord }}=\mathbf{W}^{\text {ord }} \times \mu^{\text {ord }}
$$

for finite groups $\mu$ and $\mu^{\text {ord }}$. Then we have that

$$
\mathbf{W} \cong \mathbb{Z}_{p}^{1+[F: \mathbb{Q}]+d} \quad \text { and } \quad \mathbf{W}^{\text {ord }} \cong \mathbb{Z}_{p}^{1+d},
$$

where $d$ is the defect of the Leopoldt conjecture for $F$ and $p$, that is, $d=$ $\operatorname{rank}_{\mathbb{Z}} E_{U}-\operatorname{rank}_{\mathbb{Z}_{p}} \bar{E}_{U}$. The completed group algebra $\Lambda=\mathcal{O}[[\mathbf{W}]]$ (resp. $\Lambda^{\text {ord }}=$ $\left.\mathcal{O}\left[\left[\mathbf{W}^{\text {ord }}\right]\right]\right)$ with respect to the adic topology under the augmentation ideal is noncanonically isomorphic to the power series ring of $r$ variable with coefficients in $\mathcal{O}$ for $r=1+[F: \mathbb{Q}]+d$ (resp. $r=1+d$ ). We have $\mathcal{O}[[\mathbf{G}]] \cong \Lambda[\mu]$ and $\mathcal{O}\left[\left[\mathbf{G}^{\text {ord }}\right]\right] \cong \Lambda^{\text {ord }}\left[\mu^{\text {ord }}\right]$. If $V \subset V^{\prime}$, there is a surjective $\mathcal{O}[[\mathbf{G}]]$-algebra homomorphism of the Hecke algebra for $V^{\prime}$ onto the Hecke algebra for $V$, which takes $\mathbb{}(y)$ to $\mathbb{T}(y)$. We then put

$$
\begin{aligned}
h_{\kappa} & =h_{\kappa, U}(\mathcal{O})=\lim _{V} h_{\kappa}(V ; \mathcal{O}), \\
h_{\kappa}^{\text {n.ord }} & =h_{\kappa, U}^{n . o r d}(\mathcal{O})=\varliminf_{\longleftarrow} h_{\kappa}^{\text {n.ord }}(V ; \mathcal{O}),
\end{aligned}
$$

where the limit is taken with respect to open subgroups $V$ of $U_{0}(p)$ satisfying $V \supset U_{1}^{1}\left(p^{\infty}\right)=\bigcap_{\alpha} U_{1}^{1}\left(p^{\alpha}\right)$. When $\kappa \in \mathscr{A}^{\text {ord }}$, we write $h_{\kappa}^{\text {ord }}$ in place of $h_{\kappa}^{\text {n.ord }}$. Restricting the $V$ 's to those containing $U_{1}\left(p^{\infty}\right)=\bigcap_{\alpha} U_{1}\left(p^{\alpha}\right)$, we define for $\kappa \in \mathscr{A}^{\text {ord }}$ the ordinary $p$-adic Hecke algebra as

$$
h_{\kappa}^{\text {ord }}=h_{\kappa, U}^{\text {ord }}(\mathcal{O})=\lim _{V} h_{\kappa}^{\text {ord }}(V ; \mathcal{O}) .
$$

Then we have natural projections: $h_{\kappa} \rightarrow h_{\kappa}^{n . o r d} \rightarrow h_{\kappa}^{\text {ord }}$ taking $\mathbb{T}(y)$ to $\mathbb{T}(y)$ and compatible with the projection: $\mathbf{G} \rightarrow \mathbf{G}^{\text {ord }}$. When $U=\widehat{\Gamma}_{1}(N)$, we use the symbol like $h_{\kappa}\left(N p^{\infty} ; \mathcal{O}\right)$ for these algebras. Then writing $\mathbf{h}_{\kappa}$ for any one of $h_{\kappa}, h_{\kappa}^{n . o r d}$ and $h_{\kappa}^{\text {ord }}$, we have

(Univ)

$$
\mathbf{h}_{\kappa} \cong \mathbf{h}_{\kappa^{\prime}} \quad \text { as long as } \kappa>0 \text { and } \kappa^{\prime}>0 \text {. }
$$


These isomorphisms are $\mathbb{O}[[\mathbf{G}]]$-algebra isomorphisms and take $\mathbb{T}(y)$ to $\mathbb{T}(y)$ and $\langle\mathfrak{a}\rangle$ to $\langle\mathfrak{a}\rangle$. The assertions (Univ) is proven in [35] Theorem 2.3 and [34] Theorems 3.2 and 3.3. Because of (Univ), we omit the subscript " $\kappa$ " from the notation. Now we state the control theorem:

$$
\begin{gathered}
h_{U}^{\text {n.ord }} \otimes_{\mathcal{O}[[\mathbf{G}]], \kappa} K \cong h_{\kappa}^{\text {n.ord }}\left(U_{0}\left(p^{\alpha}\right) ; K\right) \quad(0<\kappa \in \mathscr{A}), \\
h_{U}^{\text {ord }} \otimes_{\mathcal{O}\left[\left[\mathbf{G}^{\text {ord }}\right]\right], \kappa} K \cong h_{\kappa}^{\text {ord }}\left(U_{0}\left(p^{\alpha}\right) ; K\right) \quad\left(0<\kappa \in \mathscr{A}^{\text {ord }}\right) .
\end{gathered}
$$

Here we regard $K$ as an $\mathcal{O}[[\mathbf{G}]]$-algebra via the algebra homomorphism $\kappa: \mathcal{O}[[\mathbf{G}]] \rightarrow$ $K$ induced by the arithmetic character $\kappa$, and we implicitly supposed that $\varepsilon_{\kappa}$ factors through $T\left(\mathbb{Z} / p^{\alpha} \mathbb{Z}\right)$. This has been proven in [35] Theorem 2.4 (resp. [34] Theorem 3.4) for $h^{\text {n.ord }}$ (resp. $h^{\text {ord }}$ ). The control theorem holds over $\mathcal{O}$ in place of $K$ if $F=\mathbb{Q}$, and we conjecture that this stronger version should hold for general base field $F$. Under a certain circumstance, the stronger version of (Ctl) for general $F$ follows from a recent result of Fujiwara [25].

\subsection{Duality between Hecke algebras and $p$-adic modular forms}

The projection maps $h_{\kappa}(W ; A) \rightarrow h_{\kappa}(V ; A)(V \supset W)$ is induced by restricting the operator $h$ in $h_{\kappa}(W ; A)$ to the subspace $S_{\kappa}(V ; A) \subset S_{\kappa}(W ; A)$. Thus by definition, $\mathbf{h}$ naturally acts on $\bigcup_{V} S_{\kappa}(V ; A)$. Here the union is taken, via $q$-expansion, inside the space of functions on $F_{\mathbb{A}^{(}(\infty)}^{\times}$with values in the completed monoid ring $A\left[\left[q^{\xi}\right]\right]\left(\cong A\left[\left[F_{+}\right]\right]\right)$. We even consider the sum $\sum_{\kappa}\left(\bigcup_{V} M_{\kappa}(V ; A)\right)$ over all positive arithmetic weights $\kappa$ in the space of $q$-expansions. We then define a $p$-adic Banach norm on $\sum_{\kappa}\left(\bigcup_{V} M_{\kappa}(V ; \Omega)\right)$ by

$$
|f|_{p}=\operatorname{Sup}_{y}\left(\left|\mathbf{a}_{0, p}(y, f)\right|_{p},\left|\mathbf{a}_{p}(y, f)\right|_{p}\right) .
$$

By (T1-2) in Section 2.4, each element $h$ of the Hecke algebras gives a bounded operator under the above norm. Thus the action of Hecke algebras extends to the completions under the norm. Let $V_{\infty}=U_{1}^{1}\left(p^{\infty}\right)$ or $U_{1}\left(p^{\infty}\right)$, and for each closed O-algebra $A$ in $\Omega$, we write $S_{\kappa}\left(V_{\infty} ; A\right)$ for the completion of $\bigcup_{V \supset V_{\infty}} S_{\kappa}(V ; A)$ under the above Banach norm. Similarly we write $M\left(V_{\infty} ; A\right)$ (resp. $M_{\kappa}\left(V_{\infty} ; A\right)$ ) for the completion of $\sum_{\kappa}\left(\bigcup_{V \supset V_{\infty}} M_{\kappa}(V ; A)\right)$ (resp. $\left.\bigcup_{V \supset V_{\infty}} M_{\kappa}(V ; A)\right)$. By definition, the Hecke algebra of each space acts faithfully on the space. Write $\operatorname{simply} S$ for $S_{\kappa}(V ; A)$. Here we allow $V$ to be $V_{\infty}$. Then we write $h(S)$ for the corresponding Hecke algebra embedded in $\operatorname{End}_{A}(S)$. Using this notation, we define a pairing

$$
\langle,\rangle: h(S) \times S \rightarrow A \quad \text { by } \quad\langle h, f\rangle=\mathbf{a}_{p}(1, f \mid h) .
$$

Then it is known (e.g. [41] Section 3) that

$$
h(S) \cong \operatorname{Hom}_{A}(S, A) \quad \text { and } \quad S \cong \operatorname{Hom}_{A}(h(S), A) .
$$


Then the dual version of (Univ) is as follows: for $\kappa>0$ and $\kappa^{\prime}>0$,

$\left(\mathrm{Univ}^{*}\right) \quad S_{\kappa, U}^{n . \text { ord }}=S_{\kappa^{\prime}, U}^{n . \text { ord }}, \quad S_{\kappa, U}^{\text {ord }}=S_{\kappa^{\prime}, U}^{\text {ord }}, \quad S_{\kappa}\left(U_{1}^{1}\left(p^{\infty}\right) ; \mathcal{O}\right)=S_{\kappa^{\prime}}\left(U_{1}^{1}\left(p^{\infty}\right) ; \mathcal{O}\right)$,

where $S_{\kappa, U}^{n . o r d}=e S_{\kappa}\left(U_{1}^{1}\left(p^{\infty}\right) ; \mathcal{O}\right)$ and $S_{\kappa, U}^{\text {ord }}=e S_{\kappa}\left(U_{1}\left(p^{\infty}\right) ; \mathcal{O}\right)$, etc. The equality in (Univ*) means that the left-hand side and the right-hand side coincide in the space of functions on $F_{A(\infty)}^{\times}$with values in $\mathcal{O}\left[\left[q^{\xi}\right]\right]$. For these spaces independent of weight, we omit the subscript " $\kappa$ " from the notation. For each G-module $M$, we write $M[\chi]$ for the subspace of $M$ on which $\mathbf{G}$ acts via its character $\chi$. Then the dual version of $(\mathrm{Ctl})$ is given by

$$
\begin{aligned}
S_{U}^{n . o r d}[\kappa] & =S_{\kappa}^{\text {n.ord }}\left(U_{0}\left(p^{\alpha}\right) ; 0\right) \quad(0<\kappa \in \mathscr{A}), \\
S_{U}^{\text {ord }}[\kappa] & =S_{\kappa}^{\text {ord }}\left(U_{0}\left(p^{\alpha}\right) ; \mathcal{O}\right) \quad\left(0<\kappa \in \mathscr{A}^{\text {ord }}\right) .
\end{aligned}
$$

Since (S2) is equivalent to (M2) when $n(\kappa) \notin \mathbb{Z} t, M_{\kappa}\left(V_{\infty} ; \mathcal{O}\right)=S_{\kappa}\left(V_{\infty} ; \mathcal{O}\right)$ for such $\kappa$. Contrary to this, $M_{\kappa}\left(V_{\infty} ; \mathfrak{O}\right)$ is really bigger than $S_{\kappa}\left(V_{\infty} ; \mathfrak{O}\right)$ if $n(\kappa) \in \mathbb{Z} t$. We conjecture that $M_{\kappa}\left(V_{\infty} ; \mathcal{O}\right)$ is independent of $\kappa$ as long as $n(\kappa) \in \mathbb{Z} t$ and coincides with $M\left(V_{\infty} ; \mathcal{O}\right)$. This fact probably follows from a detailed analysis of Eisenstein series combined with (Univ*). Anyway, we do not need the independence of weight for $M_{\kappa}\left(V_{\infty} ; \mathcal{O}\right)$. Since the Hecke operator $\mathbb{T}(p)$ continuously acts on $M\left(V_{\infty} ; \mathcal{O}\right)$, we can think of $M^{\text {n.ord }}\left(V_{\infty} ; \mathcal{O}\right)$ and $M^{\text {ord }}\left(V_{\infty} ; \mathcal{O}\right)$.

\subsection{Modular parametrization by $\mathscr{X}$}

For each linear map $\phi: h\left(N p^{\infty} ; \mathcal{O}\right) \rightarrow \mathcal{O}$, we have by the duality $f_{\phi} \in S\left(N p^{\infty} ; \mathcal{O}\right)$ such that $\left\langle h, f_{\phi}\right\rangle=\phi(h)$. By (T2) in Section 2.4, we see $\mathbf{a}_{p}\left(y, f_{\phi}\right)=\phi(\mathbb{T}(y))$ for integral ideles $y$. Since $\mathbf{a}_{p}$ is supported on integral ideles (see Section 2.3), the value of $\mathbf{a}_{p}$ is completely determined by $\phi$. It is easy to see that $f_{\phi}$ is a common eigenform of all Hecke operators $\mathbb{T}(y)$ if and only if $\phi$ is an $\mathbf{0}$-algebra homomorphism. Thus we get a parametrization of all $\mathcal{O}$-integral common eigenforms in $S\left(N p^{\infty} ; \mathcal{O}\right)$ by the O-valued points of $\operatorname{Spec}(h)$ for $h=h\left(N p^{\infty} ; 0\right)$. In particular, the closed subschemes $X=\operatorname{Spec}\left(h^{\text {n.ord }}\right)$ and $Y=\operatorname{Spec}\left(h^{\text {ord }}\right)$ parameterize eigenforms in $S^{n . o r d}\left(N p^{\infty} ; \mathcal{O}\right)$ and $S^{\text {ord }}\left(N p^{\infty} ; \mathcal{O}\right)$, respectively. The structure of the schemes $X$ and $Y$ are fairly simple, since we know from [34] Theorem 3.3 and [35] Theorem 2.4 that

(F) $\quad h^{\text {n.ord }}\left(N p^{\infty} ; \mathcal{O}\right) \quad\left(\right.$ resp. $\left.h^{\text {ord }}\left(N p^{\infty} ; \mathcal{O}\right)\right)$ is finite and torsion-free over $\Lambda=$ $\mathcal{O}[[\mathbf{W}]]\left(\right.$ resp. $\left.\Lambda^{\text {ord }}=\mathcal{O}\left[\left[\mathbf{W}^{\text {ord }}\right]\right]\right)$.

The space $\mathscr{X}\left(\overline{\mathbb{Q}}_{p}\right)$ of $\overline{\mathbb{Q}}_{p}$-valued points of $\operatorname{Spec}(\Lambda)$ is isomorphic to the product of some copies of the open unit disk in $\overline{\mathbb{Q}}_{p}$, and the scheme $X$ is a finite covering of $\mathscr{X}$.

There is another way to describe the parametrization of common eigenforms by each irreducible component of the Hecke algebra. To simplify the notation, we just 
write $\mathbf{h}(\operatorname{resp} . \boldsymbol{\Lambda})$ for one of $h^{n . o r d}\left(N p^{\infty} ; \mathcal{O}\right)$ and $h^{\text {ord }}\left(N p^{\infty} ; \mathcal{O}\right)\left(\operatorname{resp} . \Lambda\right.$ and $\left.\Lambda^{\text {ord }}\right)$. Let $\mathbb{L}$ be the quotient field of $\boldsymbol{\Lambda}$. We take a finite extension $\mathbb{K}$ of $\mathbb{L}$ and a $\boldsymbol{\Lambda}$-algebra homomorphism $\lambda: \mathbf{h} \rightarrow \mathbb{K}$. Since $\mathbf{h}$ is finite over $\boldsymbol{\Lambda}, \lambda$ has values in the integral closure $\mathbb{Q}$ in $\mathbb{K}$ of $\boldsymbol{\Lambda}$, which is finite over $\boldsymbol{\Lambda}$. Then enlarging $\mathbb{O}$ if necessary, we may

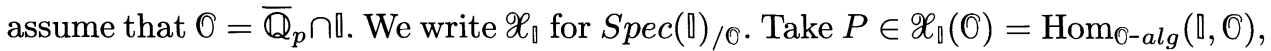
and compose it with $\lambda$ getting $\lambda_{P}=P \circ \lambda$ which is a point of $X(\mathcal{O})$. Then we get a common eigenform $f(P) \in \mathbb{S}$ such that $\mathbf{a}_{p}(y, f(P))=\lambda_{P}(\mathbb{T}(y))$ for integral $y$, where we have written $\mathbb{S}$ for one of $S^{n . o r d}\left(N p^{\infty} ; \mathcal{O}\right)$ and $S^{\text {ord }}\left(N p^{\infty} ; \mathcal{O}\right)$ according to our choice of $\mathbf{h}$. We write for each closed 0 -subalgebra $A$ of $\Omega$

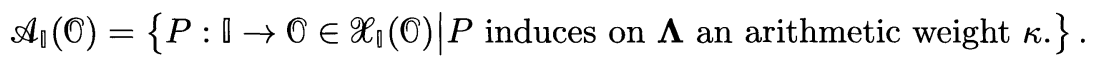

We write $\kappa(P)$ for the arithmetic weight induced by $P \in \mathscr{A}_{\mathbb{1}}(\mathcal{O})$. Then for positive $P \in \mathscr{A}_{\mathbb{1}}(\mathcal{O})$ (that is, $\left.\kappa(P)>0\right), f(P)$ is classical by $\left(\mathrm{Ctl}^{*}\right.$ ) (see [34] Corollary 3.5 and [35] Corollary 2.5). Here we say a modular form $f \in \mathbb{S}[\kappa]$ "classical" if there exists a modular form $f^{\circ} \in S_{\kappa}\left(N p^{\alpha} ; \overline{\mathbb{Q}}\right)$ for some $\alpha$ such that $i_{p}^{-1}\left(\mathbf{a}_{p}(y, f) y_{p}^{n}\right)=a\left(y, f^{\circ}\right)$ for all integral $y$. Then we can think of a formal $q$-expansion $f \in \mathbb{Q}\left[\left[q^{\xi}\right]\right]$ given by

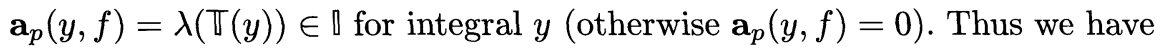

$$
f(P)(y)=P(f(y))=\sum_{\xi} P\left(\mathbf{a}_{p}(\xi y d, f)\right) q^{\xi} .
$$

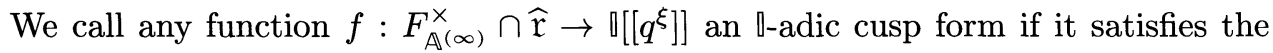
following properties:

$f(P)$ is a p-adic modular form in $S\left(N p^{\infty} ; \mathcal{O}\right)[\kappa(P)]$;

(ii) For Zariski densely populated points of $\mathscr{A}_{\mathbb{1}}(\Omega)$ in $\mathscr{X}_{\mathbb{1}}, f(P)$ is a classical cusp form.

We call an -adic form is nearly ordinary (resp. ordinary) if $\mathbf{h}=h^{\text {n.ord }}$ and $f(P) \in S^{\text {n.ord }}$ (resp. $\mathbf{h}=h^{\text {ord }}$ and $f(P) \in S^{\text {ord }}$ ) for all $P$. It is easy to check (e.g. [33] Section 7.3) that

$\operatorname{Hom}_{\Lambda}\left(h^{\text {n.ord }}\left(N p^{\infty} ; \mathcal{O}\right), \mathbb{}\right) \cong\left\{\right.$-adic nearly ordinary forms of level $\left.N p^{\infty}\right\}$ and

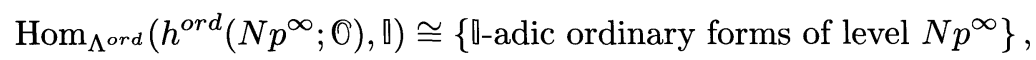

where the isomorphisms are given by $\phi \mapsto\left(y \mapsto \sum_{\xi} \phi(\mathbb{T}(\xi y d)) q^{\xi}\right)$.

\subsection{Galois representations attached to $\lambda$}

Fix an algebraic closure $\bar{F}$ of $F$. For a finite dimensional vector space $V$ over $\mathbb{K}$,

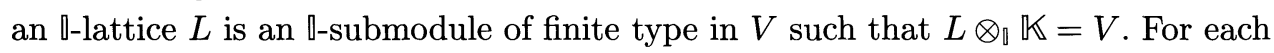


$\lambda: \mathbf{h} \rightarrow \mathbb{\mathbb { l }}$, we have a Galois representation

$$
\pi(\lambda): \mathscr{G}_{F}=\operatorname{Gal}(\bar{F} / F) \rightarrow G L(V(\lambda))
$$

for a two dimensional vector space $V(\lambda)$ over $\mathbb{K}$ satisfying the following properties:

(i) There is an $\square$-lattice $L$ in $V(\lambda)$ stable under $\pi(\lambda)$, and $\pi(\lambda)$ is a continuous map into $\operatorname{Aut}_{\mathbb{1}}(L)$ equipped with the adic topology of the maximal ideal of $\square$;

(ii) $\pi(\lambda)$ is irreducible and is unramified outside $N p$;

(iii) For each prime $\mathfrak{l}$ outside $N p$ and for the geometric Frobenius $\phi_{\mathfrak{l}}$

$$
\operatorname{det}\left(1-\pi(\lambda)\left(\phi_{\mathfrak{l}}\right) X\right)=1-\lambda(\mathbb{T}(\mathfrak{l})) X+\langle\mathfrak{l}\rangle X^{2}
$$

(iv) For the place $\mathfrak{R}$ in $F$ induced by $i_{p} \sigma$, writing $\mathscr{G}_{F_{\mathfrak{B}}}=\operatorname{Gal}\left(\bar{F}_{\mathfrak{\beta}} / F_{\mathfrak{\beta}}\right)$ for the decomposition group at $\mathfrak{R}$, there is a one dimensional subspace $V_{\mathfrak{R}}^{-}=V_{\mathfrak{B}}^{-}(\lambda)$ of $V(\lambda)$ stable under $\pi\left(\mathscr{G}_{F_{\mathfrak{\beta}}}\right)$ such that $\mathscr{G}_{F_{\mathfrak{\beta}}}$ acts on $V_{\mathfrak{B}}^{-}$via the character $\delta: F_{\mathfrak{B}}^{\times} \subset \mathscr{G}_{F_{\mathfrak{\beta}}}^{a b} \rightarrow \mathbb{1}^{\times}$given by $\delta(y)=\lambda(\mathbb{T}(y))$,

where $\mathscr{G}_{F_{\mathfrak{\beta}}}^{a b}$ is the maximal abelian (continuous) quotient of $\mathscr{G}_{F_{\mathfrak{R}}}$ and $F_{\mathfrak{\beta}}^{\times}$is viewed as a subgroup of $\mathscr{G}_{F_{\mathfrak{B}}}^{a b}$ via local class field theory. This fact is proven in [38] using Wiles' technique of pseudo representations. Actually we have taken the dual of the representation constructed in [38]. Thus in [38], the character $\delta$ as above appeared on a one-dimensional quotient of the representation space, but the character $\delta$ itself is unchanged because the identification $F_{\mathfrak{\beta}}^{\times} \subset \mathscr{G}_{F_{\mathfrak{B}}}^{a b}$ is given by assigning to each prime element the geometric Frobenius element instead of arithmetic Frobenius in [38]. In particular, the assertion (iv) tells us that $\pi(\lambda)\left(\mathscr{G}_{F_{\mathfrak{B}}}\right)$ is contained in a split Borel subgroup of $G L_{2}(\mathbb{K})$, and $\lambda$ factors through $h^{\text {n.ord }} \otimes_{\mathcal{O}[[\mathbf{G}]]} \mathcal{O}\left[\left[\mathbf{G}^{\text {ord }}\right]\right]$ if and only if $\delta$ is unramified. We have a natural projection $\rho: h^{\text {n.ord }} \otimes_{\mathcal{O}[[\mathbf{G}]]} \mathcal{O}\left[\left[\mathbf{G}^{\text {ord }}\right]\right] \rightarrow h^{\text {ord }}$ and can show that the localization of $\operatorname{Ker}(\rho)$ at positive $P \in \mathscr{A}^{\text {ord }}$ vanishes. Thus $\operatorname{Ker}(\rho)$ is a torsion $\Lambda^{\text {ord }}$-module. In particular, $\lambda$ is ordinary (that is, factoring through $h^{\text {ord }}$ ) if and only if $\delta$ is unramified. When $F=\mathbb{Q}$, it is known [38] (iv) p.119 that $\rho$ is an isomorphism.

For each $P \in \mathscr{Q}_{\mathbb{1}}(K)$, a Galois representation $\pi\left(\lambda_{P}\right): \mathscr{G}_{F} \rightarrow G L_{2}(K)$ is called a residual representation of $\pi(\lambda)$ modulo $P$ if $\pi\left(\lambda_{P}\right)$ is semi-simple and $\operatorname{Tr}\left(\pi\left(\lambda_{P}\right)(\sigma)\right)$ is equal to $P(\operatorname{Tr}(\pi(\lambda)(\sigma)))$ for all $\sigma \in \mathscr{G}_{F}$. If a residual representation exists, then it is unique up to $K$-isomorphisms. Again by Wiles' technique, one can show the existence of $\pi\left(\lambda_{P}\right)$ ([38] Theorem I). If once we know the existence of $\pi\left(\lambda_{P}\right)$, it is easy to show that the assertions (ii) (iii) and (iv) hold replacing $\mathbb{K}$ by $K$ and $\lambda$ by $\lambda_{P}=P \circ \lambda$, because the semi-simplification of the restriction of $\pi\left(\lambda_{P}\right)$ to an inertia group $I_{\mathfrak{P}}$ is the reduction modulo $P$ of the semi-simplification of the restriction of $\pi(\lambda)$ to $I_{\mathfrak{B}}$. Thus we get a parametrization of 2-dimensional Galois representations 
by $\mathscr{Q}_{\mathbb{1}}(\Omega)$. In particular, if $P$ is positive and arithmetic, $\pi\left(\lambda_{P}\right)$ is modular and hence is known to be motivic for almost all cases [6] (the exceptional cases only occur when $m(\kappa)=n(\kappa)+t)$. Here the phrase "a modular Galois representation" means that it is associated to a classical common eigenform of all Hecke operators in the manner described in (iii) above. Modular Galois representations are constructed by Taylor [69] and by Blasius and Rogawski [6] independently. The weight $\kappa$ of a modular Galois representation $\varphi$ gives the Hodge type of the attached motive (cf. $\left(\mathrm{A} 3^{\prime}\right)$ ), that is, $\left(m_{\sigma}, n_{\sigma}\right)$ and $\left(n_{\sigma}, m_{\sigma}\right)$ for each $\sigma \in I$, and the motivic weight of $\varphi$ is given by $\{\kappa\}=[m+n]$ if $\varphi$ is modular of weight $\kappa$.

\subsection{Congruence modules and differential modules}

To describe congruence among cusp forms in terms of Hecke algebras and deformation rings of Galois representations, we here introduce a general notion of congruence modules and differential modules: Let $R$ be an algebra over a normal integral domain $A$. We assume that $R$ is an $A$-torsion-free module of finite type. Let $\phi: R \rightarrow A$ be an $A$-algebra homomorphism. We define $C_{1}(\phi ; A)=\Omega_{R / A} \otimes_{R, \phi} \operatorname{Im}(\phi)$, which we call the differential module of $\phi$. We suppose that the total quotient ring $\operatorname{Frac}(R)$ can be decomposed uniquely into $\operatorname{Frac}(R)=\operatorname{Frac}(\operatorname{Im}(\phi)) \times \mathbb{X}$ as an algebra direct product. Let $\mathfrak{a}=\operatorname{Ker}(R \rightarrow \mathbb{X})$. Then we put $C_{0}(\phi ; A)=(R / \mathfrak{a}) \otimes_{R, \phi} \operatorname{Im}(\phi) \cong$ $\operatorname{Im}(\phi) /(\operatorname{Im}(\phi) \cap R)([39]$ Lemma 6.3), which is called the congruence module of $\phi$ but is actually a ring. Here the intersection $\operatorname{Im}(\phi) \cap R$ is taken in $\operatorname{Frac}(R)$. Suppose now that $A$ is a subring of a number field in $\overline{\mathbb{Q}}$. Since $\operatorname{Spec}\left(C_{0}(\phi ; A)\right)$ is the scheme theoretic intersection of $\operatorname{Spec}(\operatorname{Im}(\phi))$ and $\operatorname{Spec}(R / \mathfrak{a})$ in $\operatorname{Spec}(R)$, a prime $\mathfrak{p}$ is in the support of $C_{0}(\phi ; A)$ if and only if there exists an $A$-algebra homomorphism $\phi^{\prime}: R \rightarrow \overline{\mathbb{Q}}$ factoring through $R / \mathfrak{a}$ such that $\phi(a) \equiv \phi^{\prime}(a) \bmod \mathfrak{p}$ for all $a \in R$. In other words, $\phi \bmod \mathfrak{p}$ factors through $R / \mathfrak{a}$ and can be lifted to $\phi^{\prime}$. We see easily that $\operatorname{Supp}_{A}\left(C_{0}(\phi ; A)\right)=\operatorname{Supp}_{A}\left(C_{1}(\phi ; A)\right)$. By definition, we see that $C_{0}(\phi ; A) \cong A / \eta(\phi)$ for an ideal $\eta(\phi)$ if $\operatorname{Im}(\phi)=A$. When $\operatorname{Hom}_{A}(R, A)$ is $R$-free of rank 1 (if $R$ and $A$ are both Gorenstein rings), $\eta(\phi)$ is principal. By abusing notation, we also write $\eta(\phi)$ for the generator of the principla ideal.

We continue to write $\mathbf{h}$ for one of $h^{\text {n.ord }}\left(N p^{\infty} ; \mathcal{O}\right)$ and $h^{\text {ord }}\left(N p^{\infty} ; \mathcal{O}\right)$. We first

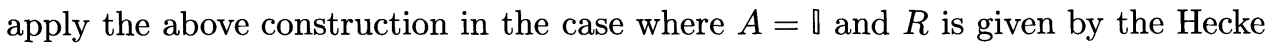
algebra $\mathbf{h}$. Let $\lambda: \mathbf{h} \rightarrow \llbracket$ be a $\boldsymbol{\Lambda}$-algebra homomorphism. We call $\lambda$ primitive (of conductor $N)$ if $\lambda$ has minimal prime-to- $p$ level $N$ among $\lambda^{\prime}: h^{n \text {.ord }}\left(D p^{\infty} ; 0\right)$ or

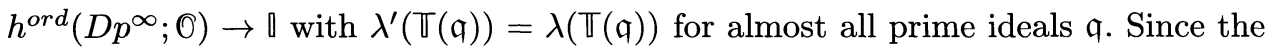
theory of new forms is readily transmitted to -adic nearly ordinary and ordinary forms, if $\lambda$ is primitive, the irreducible component of $\operatorname{Spec}(\mathbf{h})$, through which $\lambda$ factors, is reduced ([34] Theorem 3.6). Note that $\mathbf{h}$ is not only a $\boldsymbol{\Lambda}$-algebra but also 
an $\mathscr{O}\left[\left[G^{\prime}\right]\right]$-algebra, where $G^{\prime}=\mathbf{G}$ or $\mathbf{G}^{\text {ord }}$ according as $\mathbf{h}=h^{\text {n.ord }}$ or $h^{\text {ord }}$. Note that $G^{\prime}=T_{1}\left(\mathbb{Z}_{p}\right) \times \mathbf{G}^{\text {ord }}$ if $\mathbf{h}=h^{n \text {.ord }}$. Moreover the action of $\mathbf{G}^{\text {ord }} \subset G^{\prime}$ is just the action of the center of $G\left(\mathbb{Z}_{p}\right)$. Thus we can extend the action of $\mathbf{G}^{\text {ord }}$ to the action of the center $Z(\mathbb{A})$ of $G(\mathbb{A})$. It is known that the action of $Z(\mathbb{A}) \cong F_{\mathbb{A}}^{\times}$factors through

$$
\bar{Z}=\bar{Z}(N)=F_{\AA}^{\times} / \overline{F^{\times} U_{F}\left(N p^{\infty}\right) F_{\infty}^{\times}},
$$

where $U_{F}\left(N p^{\alpha}\right)=U_{1}\left(N p^{\alpha}\right) \cap Z\left(A^{(\infty)}\right)$ and $U_{F}\left(N p^{\infty}\right)=\bigcap_{\alpha>0} U_{F}\left(N p^{\alpha}\right)$. Then we can decompose $\bar{Z}=\mathbb{W}^{\text {ord }} \times \bar{Z}_{\text {tor }}$ for a torsion free subgroup $\mathbb{W}^{\text {ord }}\left(\supset \mathbf{W}^{\text {ord }}\right.$ as an open subgroup) and a finite group $\bar{Z}_{\text {tor }}$. Thus we get a group $\mathbb{G}$ a bit bigger than $G^{\prime}$ such that $\mathbb{G}=T_{1}\left(\mathbb{Z}_{p}\right) \times \bar{Z} \supset G^{\prime}$ if $\mathbf{h}=h^{\text {n.ord }}$ and $\mathbb{G}=\bar{Z}$ if $\mathbf{h}=h^{\text {ord }}$. Take the torsion-free part $\mathbb{W}$ of $\mathbb{G}$ and write $\mathbb{G}=\mathbb{W} \times \Delta$ and $\underline{\boldsymbol{\Lambda}}$ for $\mathbb{O}[[\mathbb{W}]]$. Then $\mathbf{h}$ is naturally an $\mathbb{O}[[\mathbb{G}]]$-algebra and hence is a $\underline{\boldsymbol{\Lambda}}$-algebra. Then $\lambda$ induces a character $\psi: \Delta \rightarrow \mathbb{O}^{\times}$, and we can think of the maximal quotient $\mathbf{h}(\psi)$ of $\mathbf{h}$ on which $\Delta$ acts via $\psi$. By definition, $\lambda$ factors through $\mathbf{h}(\psi)$ and gives a reduced irreducible component of

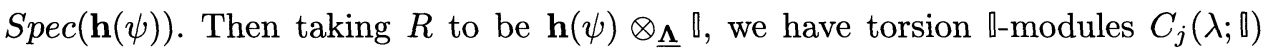

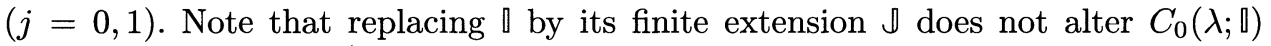

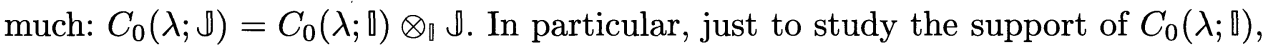

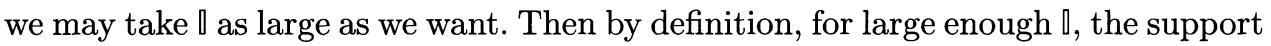

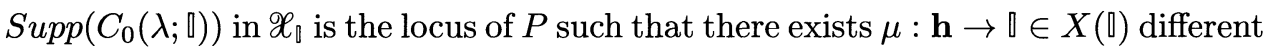
from $\lambda$ with $\lambda_{P}=\mu_{P}$. Form this, as already remarked, we have

$$
\operatorname{Supp}_{\mathbb{1}}\left(C_{0}(\lambda ; \llbracket)\right)=\operatorname{Supp}_{\mathbb{1}}\left(C_{1}(\lambda ; \llbracket)\right) .
$$

Let $P$ be an arithmetic point in $\mathscr{Q}_{\mathbb{D}}(\mathcal{O})$. Then we have $\lambda_{P}: \mathbf{h}_{P} \rightarrow \mathcal{O}$, where $\mathbf{h}_{P}$ is the maximal $\mathbb{O}$-flat quotient of $\left(\mathbf{h}(\psi) \otimes_{\underline{\Lambda}}\right.$ व) $\otimes_{\mathfrak{l}, P} \mathbb{O}$. By the control theorem $(\mathrm{Ctl})$, this algebra is isomorphic to the maximal $\mathbb{O}$-flat quotient of $h_{\kappa(P)}^{\text {n.ord }}\left(N p^{\alpha} ; \mathbb{O}\right)$ or $h_{\kappa(P)}^{\text {ord }}\left(N p^{\alpha} ; \mathcal{O}\right)$ on which $\Delta$ acts via $\psi$. Thus taking $R$ to be $\mathbf{h}_{P}$ for $A=\mathcal{O}$, we can think of finite $p$-torsion modules: $C_{j}\left(\lambda_{P} ; \mathcal{O}\right)$. By construction, it is natural to expect that $\eta(\lambda)(P)=\eta\left(\lambda_{P}\right)$ up to $\mathcal{O}$-units for all arithmetic $P$. This is proven to be true if the local component of $\mathbf{h}$ through which $\lambda$ factors is a Gorenstein ring [39].

\subsection{Non-abelian class number formulas}

In this section, we assume that $N$ is prime to $p$. The module: $C_{1}(\lambda ; \square)$ introduced in the previous section is sometimes called the analytic Mazur module of $\lambda$ and is studied in depth by Mazur-Tilouine [55], Taylor-Wiles ([73], [70]) and Fujiwara [25] in its relation to the Selmer group attached to the I-adic representation $\operatorname{Ad}(\pi(\lambda))$ (see also [49], [19] and [45]). Here we briefly describe the relation, assuming that $F=\mathbb{Q}, \mathbf{h}=h^{\text {ord }}, p \geq 5$ and that the conductor $C(\psi)$ of $\psi$ is divisible by $N$. 
Since classical notation is used in the papers quoted above; so, we first try to translate them into our language. We consider the classical space $S_{k}\left(\Gamma_{0}(N p), \psi \omega^{1-k}\right)$ of holomorphic elliptic cusp forms of weight $k$ and of "Neben"-type $\psi \omega^{1-k}$ for the Teichmüller character $\omega$. This space is isomorphic to $S_{\kappa}(N p ; \mathbb{C})[\psi]$ for $m(\kappa)=k-1$, $n(\kappa)=0$ and $\varepsilon_{\kappa}(a, d)=\psi \omega^{1-k}(d)$, where "[ $[\psi]$ " indicates the $\psi$-eigenspace under the action of $\Delta$. Since $n(\kappa)=0, \mathbb{T}((n))$ coincides with the classical Hecke operator $T(n)$ for the ideal $(n)$ generated by a positive integer $n$. Let $\mathbb{Z}\left[\psi \omega^{-m(\kappa)}\right]$ be the subalgebra of $\overline{\mathbb{Q}}$ generated by $\psi \omega^{1-k}(n)$ for all $\psi \omega^{1-k}(n)$ regarding $\psi \omega^{-m(\kappa)}$ as a Dirichlet character. Then for each $\mathbb{Z}\left[\psi \omega^{-m(\kappa)}\right]$-subalgebra $A \subset \Omega$, we write $h_{\kappa}(N p, \psi ; A)$ for the $A$-subalgebra of the linear endmorphism algebra $\operatorname{End}_{\Omega}\left(S_{\kappa}(N p ; \Omega)[\psi]\right)$ generated by Hecke operators $T(n)=\mathbb{T}((n))$ for all $n$. Then it is known [44] that

$$
h^{\text {ord }}(\psi) \otimes_{\Lambda^{\text {ord }, \kappa}} \mathcal{O} \cong h_{\kappa}^{\text {ord }}(N p, \psi ; \bigcirc)
$$

by an isomorphism taking $T(n)=\mathbb{T}((n))$ to $T(n)=\mathbb{T}((n))$. Thus for each positive arithmetic point $P \in \operatorname{Spec}(\mathbb{D})(\mathcal{O})$ with $\kappa(P)=\kappa$, we have its specialization $\lambda_{P}$ : $h_{\kappa}(N p, \psi ; \mathcal{O}) \rightarrow \mathcal{O}$. Let $f_{P}$ be a normalized Hecke eigenform in $S_{k}\left(\Gamma_{0}(N p), \psi \omega^{-m(\kappa)}\right)$ associated to $\lambda_{P}$ as described in Section 2.6. Thus $f_{P}=\sum_{n=1}^{\infty} a\left(n, f_{P}\right) q^{n}$ is a common eigenform of Hecke operators $T(n)$ for all $n$ normalized so that $f_{P} \mid T(n)=$ $a\left(n, f_{P}\right) f_{P}$ and $a\left(n, f_{P}\right)=\lambda_{P}(T(n))$. Since the conductor and the level match, the Hecke algebra $h_{\kappa}(N p, \psi ; A)$ is reduced. We now write $\mathbb{Q}\left(\lambda_{P}\right)$ for the field generated by $\lambda_{P}(T(n))$ over $\mathbb{Q}$ and write $A$ for the valuation ring $\mathbb{Q}\left(\lambda_{P}\right) \cap \mathbb{O}$ of $\mathbb{Q}\left(\lambda_{P}\right)$ with residual characteristic $p$. Thus we have well defined $C_{j}\left(\lambda_{P} ; A\right) \cong C_{j}\left(\lambda_{P} ; \mathcal{O}\right)$ $(j=0,1)$ which is a finite module. As seen in Section 2.8, we have a Galois representation $\rho=\pi\left(\lambda_{P}\right): \operatorname{Gal}(\overline{\mathbb{Q}} / \mathbb{Q}) \rightarrow G L_{2}(\mathbb{O})$ unramified outside $N p$ and $\operatorname{Tr}\left(\rho\left(F_{r o b}\right)\right)=\lambda_{P}(T(q))$ for all primes $q$ outside $N p$. Then we have the identity of the $L$-functions

$$
L\left(s, \lambda_{P}\right)=\sum_{n=1}^{\infty} \lambda_{P}(T(n)) n^{-s}=L\left(s, \pi\left(\lambda_{P}\right)\right) .
$$

Let $\pi_{P}$ be the automorphic representation of $G L_{2}(\mathbb{A})$ spanned by $f_{P}$ and its right translations. We write $L\left(s, A d\left(\lambda_{P}\right) \otimes \chi\right)$ for the $L$-function of the adjoint lift $A d\left(\pi_{P}\right)$ to $G L(3)$ [26] twisted by a Dirichlet character $\chi$. Then this $L$-function has a meromorphic continuation to the whole complex $s$-plane and satisfies a functional equation of the form $1 \leftrightarrow 1-s$ whose $\Gamma$-factor is given by

$$
\Gamma(s, A d(\lambda) \otimes \chi)=\Gamma_{\mathbb{C}}(s+\kappa-1) \Gamma_{\mathbb{R}}\left(s+\frac{\chi(-1)+1}{2}\right),
$$

where $\Gamma_{\mathbb{C}}(s)=(2 \pi)^{-s} \Gamma(s)$ and $\Gamma_{\mathbb{R}}(s)=\pi^{-s / 2} \Gamma\left(\frac{s}{2}\right)$. Thus $\Gamma(s, A d(\lambda) \otimes \chi)$ is finite at $s=0,1$ if $\chi(-1)=1$, and hence $L(1, A d(\lambda) \otimes \chi)$ is a critical value in the sense of 
Deligne and Shimura for even characters $\chi$. We shall relate the size of the module $C_{1}(\lambda ; A)$ and the $p$-primary part of the critical value $\frac{\Gamma\left(1, \operatorname{Ad}\left(\lambda_{P}\right)\right) L\left(1, \operatorname{Ad}\left(\lambda_{P}\right)\right)}{\Omega\left(+, \lambda_{P} ; A\right) \Omega\left(-, \lambda_{P} ; A\right)}$.

To define the periods $\Omega\left( \pm, \lambda_{P} ; A\right)$, we consider modular cohomology groups. We write simply $\psi_{P}$ for $\psi \omega^{-m(\kappa(P))}$. Let $L\left(n, \psi_{P} ; A\right)$ be the space of homogeneous polynomial in $(X, Y)$ of degree $n=k-2=m(\kappa)-1$ with coefficients in $A$. We let $\gamma=\left(\begin{array}{ll}a & b \\ c & d\end{array}\right) \in \Gamma_{0}(N p)$ act on $P(X, Y) \in L(n, \psi ; A)$ by

$$
(\gamma P)(X, Y)=\psi_{P}(d) P\left((X, Y)^{t} \gamma^{-1}\right)
$$

Then we can define the parabolic cohomology group $H_{c u s p}^{1}\left(\Gamma_{0}(N p), L\left(n, \psi_{P} ; A\right)\right)$ as in [64] Chapter 8 and [33] Chapter 6. We have an isomorphism

$$
\delta: S_{k}\left(\Gamma_{0}(N p), \psi_{P}\right) \oplus \bar{S}_{k}\left(\Gamma_{0}(N p), \psi_{P}\right) \cong H_{c u s p}^{1}\left(\Gamma_{0}(N p), L\left(n, \psi_{P} ; \mathbb{C}\right)\right),
$$

where $k=n+2, \bar{S}_{k}\left(\Gamma_{0}(N p), \psi_{P}\right)$ is the space of anti-holomorphic cusp forms of weight $k$ of "Neben" type character $\psi_{P}$, and $\delta$ is specified in [33] and [64] as follows: We put

$$
\omega(f)= \begin{cases}f(z)(X-z Y)^{n} d z & \text { if } f \in S_{n+2}\left(\Gamma_{0}(N p), \psi_{P}\right) \\ f(z)(X-\bar{z} Y)^{n} d \bar{z} & \text { if } f \in \bar{S}_{n+2}\left(\Gamma_{0}(N p), \psi_{P}\right)\end{cases}
$$

Then we associate to $f_{P}$ the cohomology class of the 1-cocycle $\gamma \mapsto \int_{z}^{\gamma(z)} \omega\left(f_{P}\right)$ of $\Gamma_{0}(N p)$ for a fixed point $z$ on the upper half complex plane. The map $\delta$ does not depend on the choice of $z$. There are two actions: one is a natural action of Hecke operators $T(n)$ on $H_{c u s p}^{1}\left(\Gamma_{0}(N p), L\left(n, \psi_{P} ; A\right)\right)$, and the other is an action of complex conjugation $c$ given by $c \omega(z)=\varepsilon \omega(-\bar{z})$ for $\varepsilon=\left(\begin{array}{cc}-1 & 0 \\ 0 & 1\end{array}\right)$ and a differential form $\omega$. In particular, $\delta$ and $c$ commute with $T(n)$. We write $H_{\text {cusp }}^{1}\left(\Gamma_{0}(N p), L\left(n, \psi_{P} ; A\right)\right)[ \pm]$ for the \pm -eigenspace of $c$. Then it is well known ([64] or [33]) that $H_{\text {cusp }}^{1}\left(\Gamma_{0}(N p), L\left(n, \psi_{P} ; K\right)\right)[ \pm]$ is $h_{\kappa}\left(N p, \psi_{P} ; K\right)$-free of rank 1 if $K$ is a subfield of $\overline{\mathbb{Q}}$. Recall that $A$ is a valuation ring of $\mathbb{Q}\left(\lambda_{P}\right)$. Thus

$$
H_{\text {cusp }}^{1}\left(\Gamma_{0}(N p), L\left(n, \psi_{P} ; A\right)\right)\left[\lambda_{P}, \pm\right]=A \xi_{ \pm}
$$

for a generator $\xi_{ \pm}$, where we indicate the $\lambda_{P}$-eigenspace by adding $\left[\lambda_{P}, \pm\right]$ to the notation. Then for the normalized eigenform $f_{P} \in S_{k}\left(\Gamma_{0}(N p), \psi_{P}\right)$ with $f_{P} \mid T(n)=$ $\lambda_{P}(T(n)) f$, we define $\Omega\left( \pm, \lambda_{P} ; A\right) \in \mathbb{C}^{\times}$by

$$
\delta\left(f_{P}\right) \pm c\left(\delta\left(f_{P}\right)\right)=\Omega\left( \pm, \lambda_{P} ; A\right) \xi_{ \pm} .
$$

The story on the non-abelian class number formula is a bit long; so, let us give an outline first. Via the deformation theory of Galois representations (due to Mazur, Wiles and Taylor: [54], [73] and [70]), we can identify the local ring $h$ of $h^{\text {ord }}(\psi)$ (through which $\lambda$ factors) and $h_{P}=h \otimes_{\mathbb{1}, P} \bigcirc$ with universal deformation rings classifying Galois representations (with appropriate properties) deforming $\bar{\rho}$ in good circumstances (see below for details). In this process, we know that 
1. $\quad h$ and $h_{P}$ are local complete intersections, yielding the identities:

$$
\operatorname{char}_{\rrbracket} C_{0}(\lambda ; \square)=\operatorname{char}_{\rrbracket} C_{1}(\lambda ; \square) \text { and } \# C_{0}\left(\lambda_{P} ; \mathcal{O}\right)=\# C_{1}\left(\lambda_{P} ; \mathcal{O}\right),
$$

2. $1_{P} H_{\text {cusp }}^{1}\left(\Gamma_{0}(N p), L\left(n, \psi_{P} ; \mathcal{O}\right)\right)[ \pm] \cong h_{P}$ as $h_{P}$-modules, where $\operatorname{char}_{0}(M)$ is the characteristic ideal of a torsion $\mathbb{L}$-module $M$ of finite type, and $1_{P}$ is the idempotent of $h_{P}$ in the Hecke algebra. By (2), we can compute the number $\# C_{0}(\lambda ; A)$ cohomologically, and the outcome is that it is given by the inverse of the $p$-adic absolute value of the $L$-value $\frac{\Gamma\left(1, A d\left(\lambda_{P}\right)\right) L\left(1, A d\left(\lambda_{P}\right)\right)}{\Omega\left(+, \lambda_{P} ; A\right) \Omega\left(-, \lambda_{P} ; A\right)}[43]$. By a work of Fujiwara [25], the part (1) is generalized to Hilbert modular forms under certain assumptions, although we restrict ourselves to the case where $F=\mathbb{Q}$. Moreover, again by the deformation theory [55], $C_{1}\left(\lambda_{P} ; 0\right)$ is the Pontryagin dual of the (p-adic) Selmer group $\operatorname{Sel}\left(A d\left(\pi\left(\lambda_{P}\right)\right)\right)$. In this way, we obtain the order formula of $\operatorname{Sel}\left(\operatorname{Ad}\left(\pi\left(\lambda_{P}\right)\right)\right)$ in terms of $L\left(1, A d\left(\lambda_{P}\right)\right)$. This is compatible with the conjecture of Bloch-Kato as explained in [73] Chapter 1 Section 2. A similar formula for $L\left(1, A d\left(\lambda_{P}\right) \otimes \chi\right)$ is conjectured for a general Dirichlet character $\chi$ in [19] and [46], and some evidences for the conjecture are discussed there. As already remarked, we have a generator $\eta(\lambda)$ of the annihilator of $C_{0}(\lambda ; \square)$. By the above order formula, $\eta(\lambda)(P)=\eta\left(\lambda_{P}\right)$ is equal to $\frac{\Gamma\left(1, A d\left(\lambda_{P}\right)\right) L\left(1, A d\left(\lambda_{P}\right)\right)}{\Omega\left(+, \lambda_{P} ; A\right) \Omega\left(-, \lambda_{P} ; A\right)}$ up to $\mathcal{O}$-units for all arithmetic $P$. Thus $\eta(\lambda)$ gives a $p$-adic interpolation of adjoint $L$-values up to units in 0 . Our desire in this paper is to eliminate this ambiguity of units in this $p$-adic interpolation.

To give some details to the above description, let us recall the deformation theory of Galois representations introduced by Mazur [54]. Let $\bar{\rho}=\pi\left(\lambda_{\mathfrak{m}_{0}}\right)$ be the Galois representation $\pi(\lambda) \bmod \mathfrak{m}_{\|}$for the maximal ideal $\mathfrak{m}_{\|}$of $\mathbb{\nabla}$. Thus $\bar{\rho}$ has values in $G L_{2}(\mathbb{F})$ for the residue field $\mathbb{F}$ of $\mathcal{O}$. The isomorphism class of the semi-simplification of $\bar{\rho}$ is uniquely determined by $\rho$. We consider the following condition for a number field $E \subset \overline{\mathbb{Q}}$ :

$\left(\mathrm{ai}_{E}\right)$ The restriction $\bar{\rho}_{E}$ of $\bar{\rho}$ to $\mathscr{G}_{E}$ is absolutely irreducible.

Let $\mathscr{G}(N p)=\operatorname{Gal}\left(\mathbb{Q}^{(N p)} / \mathbb{Q}\right)$ for the maximal extension $\mathbb{Q}^{(N p)} / \mathbb{Q}$ unramified outside $\{\ell \mid N p, \infty\}$. Then $\pi(\lambda)$ factors through $\mathscr{G}(N p)$. For any representation $\rho$ of $\mathscr{G}$, we write $\rho_{E}$ for the restriction of $\rho$ to $\mathscr{G}_{E}$. We consider deformations of $\bar{\rho}$ over the category $C N L_{\mathcal{O}}$ of complete local noetherian $\mathcal{O}$-algebras with residue field $\mathbb{F}$. For each object $B$ of $C N L_{\mathscr{O}}$, we write $\mathfrak{m}_{B}$ for the maximal ideal of $B$. A Galois representation $\rho: \varphi(N p) \rightarrow G L_{2}(B)$ for $B \in C N L_{\mathscr{O}}$ is a deformation of $\bar{\rho}_{E}$ if $\rho$ $\bmod \mathfrak{m}_{B}$ is identical to $\bar{\rho}_{E}$ as matrix representations. We look into the deformation

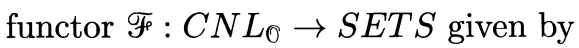

$$
\mathscr{F}(B)=\left\{\rho: \mathscr{G}^{(N p)} \rightarrow G L_{2}(B) \mid \rho \bmod \mathfrak{m}_{B}=\bar{\rho}\right\} / \approx,
$$

where " $\approx$ " is the conjugation by elements in $1+M_{2}\left(\mathfrak{m}_{B}\right)$. We impose more conditions on deformations. A deformation $\rho$ is called $p$-ordinary over a number field $E$ if 
$\left.\rho\right|_{\mathscr{D}_{\mathfrak{p}}} \cong\left(\begin{array}{cc}\delta_{\mathfrak{p}} & * \\ 0 & \varepsilon_{\mathfrak{p}}\end{array}\right)$ with an unramified character $\delta_{\mathfrak{p}}$ on every decomposition subgroup $\mathscr{D}_{\mathfrak{p}}$ of $\mathscr{G}_{E}$ at $\mathfrak{p} \mid p$. We like to impose some of the following two conditions depending on the situation:

(reg) $\rho$ is p-ordinary over $\mathbb{Q}$ with two distinct characters $\delta_{p}$ and $\varepsilon_{p}$;

By class field theory, we regard $\psi$ as a character of $G^{(N p)}$ with values in $\mathbb{O}^{\times}$. For primes $\mathfrak{l} \mid N$ outside $p$, we impose the following condition:

$(\psi) \quad \rho \cong\left(\begin{array}{ll}1 & 0 \\ 0 & \psi\end{array}\right)$ on the inertia subgroup $I_{\ell} \subset \mathscr{G}^{(N p)}$ for each prime $\ell \mid N$.

Let $\mathcal{N}$ be the $p$-adic cyclotomic character. Then $\operatorname{det}\left(\pi\left(\lambda_{P}\right)\right)=\mathcal{N}^{m(P)} \psi$. Hereafter we suppose that $p$ is prime to the order of $\psi$. We consider the following subfunctors of $\mathscr{F}$ :

$$
\begin{gathered}
\mathscr{F}_{P}(B)=\left\{\rho \in \mathscr{F}(B) \mid \rho \text { satisfies }(r e g),(\psi) \text { and } \operatorname{det}(\rho)=\mathcal{N}^{m(P)} \psi\right\}, \\
\mathscr{F}^{\text {ord }}(B)=\{\rho \in \mathscr{F}(B) \mid \rho \text { satisfies }(r e g) \text { and }(\psi)\}
\end{gathered}
$$

Under the condition that $p$ is prime to the order of $\psi,\left(\operatorname{ai}_{\mathbb{Q}}\right)$ and $(r e g)$ for $\bar{\rho}$, the functor $\mathscr{F}_{P}$ (resp. $\mathscr{F}^{\circ}{ }^{o r d}$ ) is representable by a universal couple $\left(R_{P}, \varrho_{P}\right)$ (resp. $\left(R^{\text {ord }}, \varrho^{\text {ord }}\right)$ ) (see [54], [72] and [45] Appendix).

As we have seen, under $\left(\mathrm{ai}_{\mathbb{Q}}\right)$, there is a unique Galois representation $\rho_{P}: \mathscr{G}(N p) \rightarrow$ $G L_{2}\left(h_{P}\right) \in \mathscr{F}_{P}\left(h_{P}\right)$ (resp, $\left.\rho^{\text {ord }}: \mathscr{G}^{(N p)} \rightarrow G L_{2}(h) \in \mathscr{F}^{\circ o r d}(h)\right)$ up to isomorphisms, such that $\operatorname{Tr}\left(\rho_{P}\left(F_{r o b}\right)\right)=T_{P}(\ell)\left(\right.$ resp. $\left.\operatorname{Tr}\left(\rho^{\text {ord }}\left(F r o b_{\ell}\right)\right)=T_{h}(\ell)\right)$ for all primes $\ell$ outside $N p$ (for uniqueness, see [10]), where $F_{r o b}$ is the Frobenius element in $\mathscr{G}$ at the prime $\ell$, and $T_{P}(\ell)$ (resp. $T_{h}(\ell)$ ) is the projection of $T(\ell)$ to $h_{P}$ (resp. $h$ ). It has been proven by Taylor and Wiles [73] that, under $\left(\mathrm{ai}_{\mathbb{Q}\left(\sqrt{(-1)^{(p-1) / 2} p}\right)}\right)$ and $($ reg $)$ for $\bar{\rho}, \mathscr{F}_{P}$ (resp. $\left.\mathscr{F}^{\text {ord }}\right)$ is representable by the pair $\left(h_{P}, \rho_{P}\right)$ (resp. $\left.\left(h, \rho^{\text {ord }}\right)\right)$. Thus in this case, the natural morphism $\iota_{P}: R_{P} \rightarrow h_{P}$ (resp. $\iota: R^{\text {ord }} \rightarrow h$ ) with $\iota_{P} \varrho_{P} \approx \rho_{P}$ (resp. $\iota \varrho^{\text {ord }} \approx \rho^{\text {ord }}$ ) is a surjective isomorphism. When $E$ is totally real, this result is generalized by Fujiwara [25] under certain assumptions.

In the course of the proof of the above result: $R_{P} \cong h_{P}$, it is shown that $R_{P}$ is a local complete intersection. This fact is basically equivalent to

$$
\left|C_{1}\left(\lambda_{P} ; \mathcal{O}\right)\right|=\left|C_{0}\left(\lambda_{P} ; \mathcal{O}\right)\right|
$$

By the control theorem (Ctl), this implies

$$
\operatorname{char}_{\mathbb{1}}\left(C_{1}(\lambda ; \square)\right)=(\eta(\lambda)),
$$

where for each torsion \-module $M$, its characteristic ideal $\operatorname{char}_{\llbracket}(M)$ is given by $\prod_{P} P^{\ell\left(M_{P}\right)}$ for the length $\ell\left(M_{P}\right)$ of the localization $M_{P}$ of $M$ at prime divisors $P$ 
in $\operatorname{Spec}(\mathbb{\square})$. The above fact implies that

$$
1_{P} H_{\text {cusp }}^{1}\left(\Gamma_{0}(N p), L\left(n, \psi_{P} ; \mathcal{O}\right)\right)[ \pm] \cong h_{P} \cong R_{P} \text { as } h_{P} \text {-modules }
$$

By virtue of (mlt), we can compute $C_{0}\left(\lambda_{P} ; \mathcal{O}\right)$ using cohomology groups. To explain this, we write $L(B)=H_{\text {cusp }}^{1}\left(\Gamma_{0}(N p), L\left(n, \psi_{P} ; B\right)\right)[\varepsilon]$ for $\varepsilon= \pm$. Then $L(\mathbb{O})=$ $L(A) \otimes_{A}$ O . Decomposing $\operatorname{Frac}\left(h_{\kappa}(N p, \psi ; A)\right)=\operatorname{Frac}\left(\operatorname{Im}\left(\lambda_{P}\right)\right) \times X$, we define $L^{P}(A)$ be the image of $L(A)$ in $L(A) \otimes_{h_{\kappa}(N p, \psi ; A)} \operatorname{Frac}\left(\operatorname{Im}\left(\lambda_{P}\right)\right)$ and a cohomological congruence module by

$$
C_{0}^{H}\left(\lambda_{P} ; A\right)=L^{P}(A) /\left(L(A) \cap L^{P}(A)\right) .
$$

Then (mlt) shows that

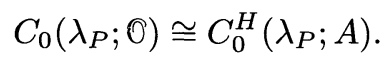

It is shown in [43] and [39] that the $p$-adic absolute value of $\frac{\Gamma\left(1, \operatorname{Ad}\left(\lambda_{P}\right)\right) L\left(1, \operatorname{Ad}\left(\lambda_{P}\right)\right)}{\Omega\left(+, \lambda_{P} ; A\right) \Omega\left(-, \lambda_{P} ; A\right)}$ is the inverse of the order of the right-hand-side module of the above equation. That is, under $\left(\mathrm{ai}_{\mathbb{Q}}\right)$ and $(r e g)$ for $\bar{\rho}$, if $p$ is outside $6 N$,

$$
\begin{gathered}
\left|\frac{c(P) \Gamma\left(1, A d\left(\lambda_{P}\right)\right) L\left(1, A d\left(\lambda_{P}\right)\right)}{\Omega(+, \lambda ; A) \Omega(-, \lambda ; A)}\right|_{p}^{-r}=\left|C_{0}^{H}\left(\lambda_{P} ; A\right)\right|=\left|C_{1}\left(\lambda_{P} ; A\right)\right| \\
\eta(\lambda)(P)=\frac{c(P) \Gamma\left(1, A d\left(\lambda_{P}\right)\right) L\left(1, A d\left(\lambda_{P}\right)\right)}{\Omega(+, \lambda ; A) \Omega(-, \lambda ; A)}
\end{gathered}
$$

where $c(P)=1$ if $\kappa(P)>t \Leftrightarrow k>2, c(P)=1-\lambda_{P}(T(p))^{-2}$ if $\kappa(P)=t \Leftrightarrow k=2$, $r=r(\mathcal{O})=\operatorname{rank}_{\mathbb{Z}_{p}} \mathcal{O}$, and ||$_{p}$ is the $p$-adic absolute value of $A$ normalized so that $|p|_{p}=p^{-1}$. Even if $p \mid N$, there is a similar formula (see [39] for details).

We now recall the definition of the Selmer group of $A d\left(\rho: G^{(N p)} \rightarrow G L_{2}(B)\right)$ over $\mathbb{Q}$ related to our modules $C_{1}$. Let $V$ be the $B$-free module of rank 2 on which $\mathscr{G}^{(N p)}$ acts via $\rho$. For each decomposition subgroup $\mathscr{D}_{p} \subset \mathscr{G}^{(N p)}$ at $p$, we write $V_{p}$ for the $\delta_{p}$ eigenspace in $V$. For primes $\ell \mid N$ prime to $p$, we write $V_{\ell}$ for the subspace fixed by the inertia at $\ell$. We identify $A d(\rho)$ with trace 0 subspace $W$ of $\operatorname{End}_{B}(V)$. We put for $\ell \mid N p$

$$
W_{\ell}=\left\{\phi \in W \mid \phi\left(V_{\ell}\right)=0\right\} .
$$

Define, for $\ell \mid N p$, writing $\Phi_{\ell}$ for the inertia subgroup at $\ell$ in $\varphi^{(N p)}$,

$$
L_{\ell}=\operatorname{Ker}\left(H^{1}\left(\mathscr{D}_{\ell}, W^{*}\right) \rightarrow H^{1}\left(\Phi_{\ell},\left(W / W_{\ell}\right)^{*}\right)\right)
$$

where $X^{*}=X \otimes_{B} B^{*}$ for the Pontryagin dual $B^{*}=\operatorname{Hom}\left(B, \mathbb{Q}_{p} / \mathbb{Z}_{p}\right)$ of $B$. Then we put

$$
\operatorname{Sel}(A d(\rho))=\bigcap_{\ell \mid N p} \operatorname{Ker}\left(H^{1}\left(\mathscr{G}^{(N p)}, W^{*}\right) \rightarrow H^{1}\left(\mathscr{D}_{\ell}, W^{*}\right) / L_{\ell}\right)
$$


It is a general fact [55] (see also [45] 3.2) that

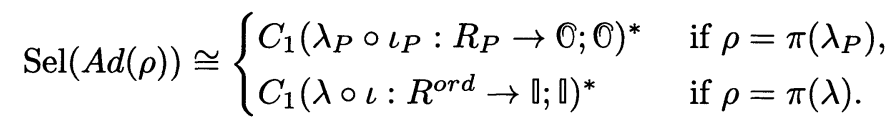

Thus combining what all we said, we get the following order formula of the Selmer group under $\left(\mathrm{ai}_{\mathbb{Q}\left(\sqrt{(-1)^{(p-1) / 2} p}\right)}\right)$ and $(p)+(6 N g)=\mathbb{Z}$ for the order $g$ of $\psi$,

$$
\begin{gathered}
\frac{\Gamma\left(1, A d\left(\lambda_{P}\right)\right) L\left(1, A d\left(\lambda_{P}\right)\right)}{\Omega\left(+, \lambda_{P} ; A\right) \Omega(-, \lambda ; A)}=\eta\left(\lambda_{P}\right) \text { up to } A \text {-units, and } \\
\operatorname{char}_{\square}(\operatorname{Sel}(A d(\pi(\lambda))))=\eta(\lambda) .
\end{gathered}
$$

This is a non-abelian generalization of a classical analytic class number formula (see [73] Chapter 4 and [49]). The definition of the Selmer group can be interpreted by Fontaine's theory, and the above formula can be viewed as an example of the Tamagawa number formula of Bloch and Kato for the motive $M\left(\operatorname{Ad}\left(\lambda_{P}\right)\right)$ yielding $\operatorname{Ad}\left(\pi\left(\lambda_{P}\right)\right)$ (see [73] p.466 and [7] Section 5). 


\section{Periods of motives}

We study $p$-adic periods of motives under the reducibility conditions $\left(\operatorname{Red}_{ \pm}\right)$of its local Galois representation at $p$-adic places. After an example of vanishing of $p$-adic periods in Section 3.3, we will prove a general non-vanishing result in Section 3.4. At the end of this chapter, we shall show that our reducibility condition $\left(\operatorname{Red}_{+}\right)$is equivalent to Panchishkin's admissibility condition when the motive is crystalline (Section 3.5).

\subsection{Periods of motives}

Let $M$ be a pure motive of rank $n$ defined over $F$ with coefficients in $E$. Morphisms in the category of motives we treat are induced from absolute Hodge cycles. We always assume that $E$ is a subfield of $\overline{\mathbb{Q}}$ and that $\varphi_{F}$ acts on its etale $p$-adic realization $H_{\mathfrak{p}}(M)$ through a compatible system $\varphi$ of Galois representations. We choose an embedding $\iota: \overline{\mathbb{Q}} \hookrightarrow \mathbb{C}$. Then $E$ can be considered to be a subfield of $\mathbb{C}$ via $\iota$. Then the $L$-function of $M$ or $\varphi$ is defined by

$$
L_{\iota}(s, \varphi)=L(s, M)=\prod_{\mathfrak{l}} \operatorname{det}\left(1-\left.\varphi\left(\phi_{\mathfrak{l}}\right)\right|_{H_{\mathfrak{p}}(M)^{I(\mathfrak{l})}} \mathcal{N}\left(\phi_{\mathfrak{l}}\right)^{-s}\right)^{-1},
$$

where $\mathfrak{l}$ runs over all prime ideals of $F$ (and $\mathfrak{p}$ is chosen so that $\mathfrak{l}$ is outside $N(\mathfrak{p})$ ), $I(\mathfrak{l})$ denotes an inertia group at each prime $\mathfrak{l}$ and $\phi_{\mathfrak{l}}$ denotes the geometric Frobenius class modulo $I(\mathfrak{l})$. This $L$-function is absolutely convergent for $s$ with $\operatorname{Re}(s)$ sufficiently large and is conjectured to be continued analytically to the whole $s$-plane with a functional equation:

$$
\Gamma(s, \varphi) L(s, \varphi)=\varepsilon(s, \varphi) \Gamma(1-s, \check{\varphi}) L(1-s, \check{\varphi})
$$

for a suitable exponential function $\varepsilon$ and a gamma factor $\Gamma(s, \varphi)$. When $\Gamma(0, \varphi)$ and $\Gamma(1, \check{\varphi})$ are both finite, we call $\varphi$ and $M$ critical. If $M$ is critical, there is a conjecture of Deligne [15] describing the transcendental factor $c_{\iota}^{+}(\varphi)$ of $L_{\iota}(0, \varphi)$ as follows: Write the Hodge realization as $H_{B}\left({ }^{\sigma} M\right) \otimes_{E} \mathbb{C}=\oplus_{i, j} H^{i, j}\left({ }^{\sigma} M\right)$, where ${ }^{\sigma} M$ 
is the base change of $M$ to $\mathbb{C}$ under $\iota \sigma$ for $\sigma: \bar{F} \cong \overline{\mathbb{Q}}$ and $\iota: \overline{\mathbb{Q}} \hookrightarrow \mathbb{C}$. Then the criticality of $M$ implies

(Cr) At each real place $\sigma$, complex conjugation c acts on $H^{k, j}\left({ }^{\sigma} M\right)$ via -1 (resp. +1 ) if $k=j \geq 0$ (resp. $k=j<0$ ), and $H^{k, k}\left({ }^{\sigma} M\right)=0$ for all complex place $\sigma$.

We now take a Tannakian full subcategory $\mathscr{C}_{X}$ of the category of pure absolute Hodge motives defined over $F$ with coefficients in $E$. We always assume $E$ to be a subfield of $\overline{\mathbb{Q}}$. Suppose that there exist a $\overline{\mathbb{Q}}$-algebra $X$ with a natural transformation $I_{X}$ inducing an equivalence of two fiber functors on $\mathscr{C}_{X}$ over $X$ : for each $\sigma \in I$,

$$
\begin{aligned}
\left\{M \mapsto H_{B}\left({ }^{\sigma} M\right) \otimes_{E, i}\right. & \left.X_{E}=H_{B}\left({ }^{\sigma} M\right) \otimes_{\mathbb{Q}} X\right\} \\
& \cong\left\{M \mapsto H_{D R}(M) \otimes_{F \otimes E, \sigma \otimes i} X_{E}=H_{D R}(M) \otimes_{F, \sigma} X\right\},
\end{aligned}
$$

where $i: E \hookrightarrow X_{E}=X \otimes_{\mathbb{Q}} E$ is the natural embedding. This transformation induces the comparison isomorphism:

$$
\begin{array}{r}
I_{X, \sigma}: H_{B}\left({ }^{\sigma} M\right) \otimes_{\mathbb{Q}} X=H_{B}\left({ }^{\sigma} M\right) \otimes_{E, i} X_{E} \\
\cong H_{D R}(M) \otimes_{F \otimes E, \sigma \otimes i} X_{E}=H_{D R}(M) \otimes_{F, \sigma} X .
\end{array}
$$

Here $I_{X, \sigma}$ may depends on $\sigma$ itself not just its restriction to $F$. Writing $\iota_{E}: E \hookrightarrow \overline{\mathbb{Q}}$ for the inclusion of $E$, we write $\delta_{X, \sigma}(M)$ for the $\iota_{E}$-component of $\operatorname{det}\left(I_{X, \sigma}\right)$ with respect to the basis of $H_{D R}(M)$ over $E \otimes F$ and $H_{B}\left({ }^{\sigma} M\right)$ over $E$. Here we identify $E \otimes_{\mathbb{Q}} \overline{\mathbb{Q}}$ with a product of copies of $\overline{\mathbb{Q}}$ and, the word " $\iota_{E}$-component" implies the component of $E \otimes_{\mathbb{Q}} \overline{\mathbb{Q}}$ inducing $\iota_{E}$ on $E$. We write

$$
\delta_{X}(M)=\prod_{\sigma \in I} \delta_{X, \sigma}(M)
$$

which is an element in $X^{\times}$and coincides with $\delta_{X, \sigma}\left(\operatorname{Res}_{F / \mathbb{Q}} M\right)$ up to an algebraic factor independent of $X$. Let $w \in \mathbb{Z}$ be the weight of $M$, that is, $i+j$ for $H^{i, j}(M) \neq 0$ which is independent of $(i, j)$ and $\sigma$. Then we write $\mathscr{F}^{ \pm}(M)$ for a member of the Hodge filtration $\left\{\mathscr{F}^{j} H_{D R}(M)\right\}_{j}$ such that for all $\sigma$,

$$
\begin{gathered}
\mathscr{F}^{ \pm}(M) \otimes_{F, \sigma} \mathbb{C}=\bigoplus_{i>j} H^{i, j}\left({ }^{\sigma} M\right) \quad \text { if } c \text { acts on } H^{i, i}(M) \text { by } \mp 1 \text { and } \\
\mathscr{F}^{ \pm}(M) \otimes_{F, \sigma} \mathbb{C}=\bigoplus_{i \geq j} H^{i, j}\left({ }^{\sigma} M\right) \quad \text { if } c \text { acts on } H^{i, i}(M) \text { by } \pm 1 .
\end{gathered}
$$

We put $H_{D R}^{ \pm}(M)=H_{D R}(M) / \mathscr{F}^{\mp}(M)$. When $\mathscr{F}^{+}(M)=\mathscr{F}^{-}(M)$, we simply write $\mathscr{F}(M)$ for $\mathscr{F}^{ \pm}(M)$. Let $H_{B}^{ \pm}\left({ }^{\sigma} M\right)$ be the " \pm " eigenspace of $H_{B}\left({ }^{\sigma} M\right) \oplus H_{B}\left({ }^{c \sigma} M\right)$ or $H_{B}\left({ }^{\sigma} M\right)$, according as $\sigma$ is complex or real, under the action of complex conjugation $c=c_{\iota}$ induced by $\iota$. Then under (Cr), we know from [3] or [74] (see Lemma 5.1.1 of 
Chapter 5 in the text) that if $F$ does not contain a CM field, $H_{D R}^{ \pm}(M)$ is $E \otimes F$ free, and if $H_{D R}^{ \pm}(M)$ is not $E \otimes F$-free, $F$ contains a CM-field, and there exists an automorphism $\rho$ of $F$ inducing complex conjugation on every CM-subfield of $F$ such that $H_{D R}^{ \pm}(M) \oplus H_{D R}^{ \pm}(M)^{\rho}$ is $E \otimes F$-free, where $H_{D R}^{ \pm}(M)^{\rho}$ is the $E \otimes F$-module isomorphic to $H_{D R}^{ \pm}(M)$ as an $E$-vector space on which $e \otimes f \in E \otimes F$ acts via $e \otimes \rho(f)$. Now we define the Deligne period $c_{X, \sigma, \iota}^{ \pm}(\varphi)$, following [3] and [74], by the $\iota_{E}$-component of the determinant of the following linear maps $I_{X, \sigma}^{ \pm}$with respect to basis of $H_{D R}^{ \pm}(M)$ or $H_{D R}^{ \pm}(M) \oplus H_{D R}^{ \pm}(M)^{\rho}$ over $E \otimes F$ and of $H_{B}^{ \pm}\left({ }^{\sigma} M\right)$ over $E$ : if $\iota \sigma$ is real

$$
I_{X, \sigma}^{ \pm}: H_{B}^{ \pm}\left({ }^{\sigma} M\right) \subset H_{B}\left({ }^{\sigma} M\right) \stackrel{I_{X, \sigma}}{\longrightarrow} H_{D R}(M) \otimes_{F, \sigma} X \rightarrow H_{D R}^{ \pm}(M) \otimes_{F, \sigma} X
$$

and if $\iota \sigma$ is complex,

$$
\begin{array}{r}
I_{X, \sigma}^{ \pm}: H_{B}^{ \pm}\left({ }^{\sigma} M\right) \subset H_{B}\left({ }^{\sigma} M\right) \oplus H_{B}\left({ }^{c \sigma} M\right) \stackrel{I_{X, \sigma} \oplus I_{X, c \sigma}}{\longrightarrow} \\
H_{D R}(M) \otimes_{F, \sigma \oplus c \sigma}(X \oplus X) \\
\rightarrow\left(H_{D R}^{ \pm}(M) \otimes_{F, \sigma} X\right) \oplus\left(H_{D R}^{ \pm}(M) \otimes_{F, c \sigma} X\right) .
\end{array}
$$

We regard, once and for all, $I$ as a complete representative set for $\operatorname{Hom}_{\text {alg }}(\bar{F}, \overline{\mathbb{Q}}) / \mathscr{G}_{F}$ and choose a complete representative set $\Sigma$ for $\langle c\rangle \backslash \operatorname{Hom}_{\text {alg }}(\bar{F}, \overline{\mathbb{Q}}) / \mathscr{G}_{F}$ such that $\Sigma \subset I$. When $F=\mathbb{Q}$, there is a canonical choice of $I$ made of the identity. We will make this choice always for $F=\mathbb{Q}$. We put

$$
c_{X, \iota}^{ \pm}(\varphi)=\prod_{\sigma \in \Sigma} c_{X, \sigma, \iota}^{ \pm}(\varphi)
$$

Then $c_{X, \iota}^{ \pm}(\varphi)$ is equal to $c_{X, \iota}^{ \pm}\left(\operatorname{Ind}_{\mathscr{G}_{F}}^{\mathscr{G}_{\mathbb{Q}}} \varphi\right)=c_{X, \iota}^{ \pm}\left(\operatorname{Res}_{F / \mathbb{Q}} M\right)$ up to an algebraic factor independent of $X$ (see [74] Proposition 2.2 and [42] (1.2a,b)). By definition, we have (BC) Base-change of the $E \otimes F$-basis of $H_{D R}^{ \pm}(M)$ or $H_{D R}^{ \pm}(M) \oplus H_{D R}^{ \pm}(M)^{\rho}$ by a linear transformation $\alpha \in G L_{d^{ \pm}}(F \otimes E)$ and base change of the $E$-basis of $H_{B}^{ \pm}\left({ }^{\sigma} M\right)$ by $\beta_{\sigma} \in G L_{d^{ \pm}}(E)$ transform $c_{X, \sigma, \iota}^{ \pm}(\varphi)$ into $\iota_{E} \otimes \sigma(\gamma) c_{X, \sigma, \iota}^{ \pm}(\varphi)$ for $\gamma \in(E \otimes F)^{\times}$depending only on $\alpha$ and $\beta$ but independent of $X$.

There are various choices of $X$ with particular properties. We can take $X=\mathbb{C}$. In this case, the comparison isomorphism $I_{\mathbb{C}, \sigma}$ is derived from a theorem of de Rham. The complex period $c_{\iota, \sigma}^{ \pm}(\varphi)=c_{\mathbb{C}, \sigma, \iota}^{ \pm}(\varphi)$ does not vanish, because $c$ interchanges $H^{k, j}\left({ }^{\sigma} M\right)$ and $H^{j, k}\left({ }^{\sigma} M\right)$. We write $c_{\iota}^{ \pm}(\varphi)$ for $c_{\mathbb{C}, \iota}^{ \pm}(\varphi)$. Supposing that $\varphi$ is critical, we here give a conjecture of Deligne ([15]; see also [42] Section 1) in the following form:

$$
\begin{gathered}
\frac{L_{\iota}(0, \varphi)}{c_{\iota}^{+}(\varphi)} \in \iota(\overline{\mathbb{Q}}) \text { and } \\
\iota^{-1}\left(\frac{L_{\iota}(0, \varphi)}{c_{\iota}^{+}\left(\operatorname{Ind}_{\mathscr{G}_{F}}^{\varphi_{Q}} \varphi\right)}\right)=(\iota \sigma)^{-1}\left(\frac{L_{\iota \sigma}(0, \varphi)}{c_{\iota \sigma}^{+}\left(\operatorname{Ind}_{\mathscr{G}_{F}}^{\mathscr{S}_{Q}} \varphi\right)}\right) \quad \text { for } \sigma \in \mathscr{G}_{\mathbb{Q}} .
\end{gathered}
$$




\section{$3.2 p$-Adic periods of motives}

We fix an embedding $i_{p}: \overline{\mathbb{Q}} \rightarrow \Omega$. In the $p$-adic case, various rings $X$ with the property discussed in the previous section has been studied by Fontaine. Among these, the biggest is the Fontaine's field $B_{D R}$. The field $B_{D R}$ is an algebraically closed and $p$-adically complete field extension of $\mathbb{Q}_{p}$ (and hence $\Omega$ ) with other interesting properties (see [22] for details). Here we take as $\mathscr{C}_{X}\left(X=B_{D R}\right)$ the category of de Rham motives defined in [2]. We fix an embedding of $\overline{\mathbb{Q}}_{p}$ into $B_{D R}$ so that the composite isomorphism $\overline{\mathbb{Q}}_{p} \hookrightarrow B_{D R}^{+} \stackrel{\theta}{\rightarrow} \Omega$ is the identity on $\overline{\mathbb{Q}}_{p}$, where the subring $B_{D R}^{+}$of $B_{D R}$ defined in [22] is a discrete valuation ring with residue field $\Omega$ and $\theta$ is just taking residue modulo the maximal ideal of $B_{D R}^{+}$. We write the $p$-adic etale realization of $M$ by $H_{p}(M)$. Then we assume the existence of a functorial comparison isomorphism conjectured by Fontaine, proven by Fontaine and Messing [24] under a certain condition, studied by Faltings [20] for general smooth projective varieties and further generalized to motives in $\mathscr{C}_{X}$ in [2] Theorem 0.3:

$$
I_{i_{p} \sigma, D R}: H_{p}(M) \otimes_{\mathbb{Q}} B_{D R} \cong H_{D R}(M) \otimes_{F, i_{p} \sigma} B_{D R} .
$$

Here we take the morphism defined in [20] and [2] because there seems a problem of comparison of comparison isomorphisms [50] 4.3.5. The morphism $I_{i_{p} \sigma, D R}$ is an isomorphism of $\mathscr{G}_{F_{\beta}}$-modules if we put the following Galois module structure: If the $p$-adic place $\mathfrak{R}$ is induced by $i_{p} \sigma, \mathscr{G}_{F_{\beta}}$ acts on $H_{p}(M) \otimes_{\mathbb{Q}} B_{D R}$ via the diagonal action $\tau(m \otimes b)=\tau(m) \otimes \tau(b)$ and on $H_{D R}(M) \otimes_{F, i_{p} \sigma} B_{D R}$ through $B_{D R}$, that is, $\tau(f \otimes b)=f \otimes \tau(b)$. Note that we have another functorial isomorphism coming from the identification of the Betti cohomology with the etale cohomology:

$$
I_{\iota \sigma, e t}: H_{B}\left({ }^{\sigma} M\right) \otimes_{\mathbb{Q}} \mathbb{Q}_{p}=H_{B}\left({ }^{\sigma} M\right) \otimes_{E}\left(\mathbb{Q}_{p} \otimes E\right) \cong H_{p}(M) .
$$

The etale cohomology $H_{p}(M)$ is defined by extending scalar from $F$ to $\bar{F}$. The motive ${ }^{\sigma} M$ over $\mathbb{C}$ is the scalar extension of $M$ to $\mathbb{C}$ via $\iota \sigma$. Thus $I_{\iota \sigma, e t}$ depends on $\iota \sigma: \bar{F} \cong \overline{\mathbb{Q}} \subset \mathbb{C}$. We can thus have $I_{X, \sigma}=I_{i_{p} \sigma, D R} \circ I_{\iota \sigma, e t}$ for $X=B_{D R}$. For $X=B_{D R}$, we write $c_{D R, \sigma, \iota}^{ \pm}(\varphi), c_{D R, \iota}^{ \pm}(\varphi), \delta_{D R, \sigma}(M)$ and $\delta_{D R}(M)$ for $c_{X, \sigma, \iota}^{ \pm}(\varphi)$, $c_{X, \iota}^{ \pm}(\varphi), \delta_{X, \sigma}(M)$ and $\delta_{X}(M)$, respectively. Note here that these periods are the $i_{p} \iota_{E^{-}}$ component of the determinant of the various comparison morphisms. We further note that the above period $c_{D R, \sigma, \iota}^{ \pm}(\varphi)$ may vanish depending on $\iota$ (and $i_{p}$ ). We study in detail such vanishing and non-vanishing of $p$-adic periods in the following Sections 3.3 and 3.4.

To define periods in $\Omega$ not in $B_{D R}$, we introduce now the local reducibility conditions. For that, we look into $B_{H T}$. We start recalling $\mathbb{Z}_{p}(1)$ which is the $p$-adic Tate module of $\mathbf{G}_{m}$. By identifying $\mu_{N}$ with $\mathbb{Z} / N \mathbb{Z}$ via $i_{p}\left(\iota^{-1}(\exp (2 \pi i / N))\right) \mapsto 1$, we have a trivialization: $\mathbb{Z}_{p}(1) \cong \mathbb{Z}_{p}$. We write $\mathbb{Z}_{p}(-1)$ to be the $\mathbb{Z}_{p}$-dual of $\mathbb{Z}_{p}(1)$ 
and define as a $\mathscr{G}_{\mathbb{Q}_{p}}$-module, $\mathbb{Z}_{p}( \pm n)=\mathbb{Z}_{p}( \pm 1)^{\otimes n}$ for each positive integer $n$. We put $\mathbb{Z}_{p}(0)=\mathbb{Z}_{p}$. Then we consider $\Omega(n)=\mathbb{Z}_{p}(n) \otimes_{\mathbb{Z}} \Omega$ on which $\mathscr{G}_{\mathbb{Q}_{p}}$ acts diagonally (and semi-linearly). There is a trivialization $\Omega \cong \Omega(n)$ as specified above. Since $B_{D R}^{+}$ is a valuation ring with residue field $\Omega$, we construct the associated graded algebra $B_{H T}$ of $B_{D R}$. That is canonically isomorphic to $\oplus_{n} \Omega(n)=\Omega\left[t, t^{-1}\right]$. We assume the existence of the Hodge-Tate version of $I_{i_{p} \sigma, D R}$ :

$$
I_{i_{p} \sigma, H T}: H_{p}(M) \otimes_{\mathbb{Q}_{p}} B_{H T} \cong H_{D R}(M) \otimes_{F, i_{p} \sigma} B_{H T}
$$

The existence of $I_{i_{p} \sigma, H T}$ is shown for smooth projective varieties over $\mathbb{Z}_{p}$ by Faltings [21]. Thus $\left(\mathrm{C}_{H T}\right)$ is valid at least for crystalline motives [2]. We anyway assume the existence of $I_{H T}$. We can define all periods as in Section 3.1 for $X=B_{H T}$ and $I_{X, \sigma}=I_{i_{p} \sigma, H T} \circ I_{\iota \sigma, e t}$. We express these periods replacing the subscript $X$ by $H T$ in the notation introduced in Section 3.1. Let $S_{F}$ be the set of all $p$-adic places of $F$. We suppose $\left(\mathrm{C}_{H T}\right)$ at every $\mathfrak{B} \in S_{F}$; thus, the $\iota_{E}$-component $H_{\mathfrak{p}}(M)$ of $H_{p}(M)$ is of Hodge-Tate type, where $\mathfrak{p}$ is the $p$-adic place of $E$ induced by $i_{p}$. We suppose the existence of $\mathscr{G}_{F_{\mathfrak{\beta}}}$-subrepresentation $V_{\mathfrak{B}}^{ \pm}$in $H_{\mathfrak{p}}(M)$ for each $\mathfrak{\Re}=\left\{i_{p} \sigma\right\} \in S_{F}$ such that $I_{\sigma, H T}$ induces

$\left(\operatorname{Red}_{ \pm}\right)$

$$
I_{i_{p} \sigma, H T, \mathfrak{p}}: V_{\mathfrak{B}}^{ \pm} \otimes_{E_{\mathfrak{p}}, i_{p}} B_{H T} \cong \mathscr{F}^{ \pm}(M) \otimes_{F \otimes E, \sigma \otimes i_{p}} B_{H T}
$$

As we will see later, the condition ( Red $_{-}$) is always satisfied by admissible crystalline motives in the sense of Panchishkin [56]. However this formulation is more useful, because it does not require $M$ to be crystalline. A similar condition asserting the existence of $V_{\mathfrak{B}}^{ \pm}$without reference to $\mathscr{F}^{ \pm}(M)$ is also stated in [28] as Panchishkin's condition.

We now study how the condition $\left(\operatorname{Red}_{ \pm}\right)$behaves under base change. Let $F^{\prime}$ be a subfield of $F$. Since the condition $\left(\operatorname{Red}_{ \pm}\right)$is insensitive to changing the field of definition of $M$, we may assume $F$ to be a Galois extension of $F^{\prime}$ replacing $F$ by the Galois closure of $F$ over $F^{\prime}$. Pick a prime $\mathfrak{p} \in S_{F^{\prime}}$ and decompose $\mathfrak{p}=\prod_{\mathfrak{B} \mid \mathfrak{p}} \mathfrak{B}^{e(\mathfrak{P})}$. Extending $\mathfrak{P}$ to a prime of $\bar{F}$ corresponding to $i_{p} \sigma$, let $D(\mathfrak{P})$ (resp. $D(\mathfrak{p})$ ) be the decomposition group of $\mathfrak{P}=i_{p} \sigma$ in $\mathscr{H}=\mathscr{G}_{F}$ (resp. $\mathscr{G}=\mathscr{G}_{F^{\prime}}$ ). We embed $\bigoplus_{\mathfrak{B} \mid \mathfrak{p}} \operatorname{Ind}_{D(\mathfrak{P})}^{D(\mathfrak{p})} V_{\mathfrak{P}}^{ \pm}$into $\operatorname{Ind}_{\mathscr{H}}^{\mathscr{G}} V$ as follows. We have a natural bijection between the double coset $\mathscr{H} \backslash \mathscr{G} / D(\mathfrak{P})$ and primes of $F$ dividing $\mathfrak{p}$. By fixing a complete representative set $R$ for $\mathscr{H} \backslash \mathscr{G}$, we identify $\operatorname{Ind}_{\mathscr{H}}^{\mathscr{G}} V$ with the space of functions on $R$ with values in $V$. Then $g \in \mathscr{G}$ acts on $v: R \rightarrow V$ by $g v(\sigma)=h_{\sigma} v(\tau)$, where $\sigma g=h_{\sigma} \tau$ for $\tau \in R$ and $h_{\sigma} \in \mathscr{H}$. We identify $\operatorname{Ind}_{D(\mathfrak{P})}^{D(\mathfrak{p})} V_{\mathfrak{B}}^{ \pm}$with the space of functions in $\operatorname{Ind}_{\mathscr{H}}^{\mathscr{G}} V$ supported on $\mathscr{H} \backslash \mathscr{H} \sigma D(\mathfrak{P})$ with values in $V_{\sigma(\mathfrak{B})}^{ \pm}$. It is plain from construction that this embedding is an embedding of $D(\mathfrak{p})$-modules, and $I_{i_{p} \sigma, H T}$ for $\operatorname{Res}_{F / F^{\prime}} M$, which 
is given by $\bigoplus_{\left.\tau\right|_{F^{\prime}}=\sigma} I_{i_{p} \tau, H T}$, induces an isomorphism

$$
\left(\bigoplus_{\mathfrak{B} \mid \mathfrak{p}} \operatorname{Ind}_{D(\mathfrak{P})}^{D(\mathfrak{p})} V_{\mathfrak{R}}^{ \pm}\right) \otimes_{E, i_{p}} B_{H T} \cong \mathscr{F}^{ \pm}\left(\operatorname{Res}_{F / F^{\prime}} M\right) \otimes_{F^{\prime} \otimes E, i_{p} \sigma \otimes i_{p}} B_{H T}
$$

Thus if $M$ satisfies the condition $\left(\operatorname{Red}_{ \pm}\right)$over $F, \operatorname{Res}_{F / F^{\prime}} M$ satisfies $\left(\operatorname{Red}_{ \pm}\right)$over $F^{\prime}$.

As observed in [2], if $c_{H T}^{ \pm}(\varphi)$ is supported by a single graded component, we can naturally identify the period with a $p$-adic number in $\Omega$. We now show that this is the case when $\left(\operatorname{Red}_{ \pm}\right)$is satisfied. Since the multiplication by $\delta_{H T}(\varphi)$ is the comparison isomorphism of the rank 1 motive $\bigwedge^{n} M$ and hence is supported by a single graded component. If $M$ satisfies $\left(\operatorname{Red}_{ \pm}\right)$and $\iota \sigma$ is a real place of $F, c_{H T, \sigma, \iota}^{ \pm}(\varphi)$ is the determinant of the composition of

$$
\begin{aligned}
& I_{\iota \sigma}: H_{B}^{ \pm}\left({ }^{\sigma} M\right) \otimes_{E, i_{p}} E_{\mathfrak{p}} \rightarrow H_{\mathfrak{p}}(M) / V_{\mathfrak{P}}^{\mp} \text { and } \\
& I_{i_{p} \sigma}:\left(H_{\mathfrak{p}}(M) / V_{\mathfrak{P}}^{\mp}\right) \otimes_{E, i_{p}} B_{H T} \cong\left\{H_{D R}(M) / \mathscr{F}^{\mp}(M)\right\} \otimes_{F \otimes E, \sigma \otimes i_{p}} B_{H T} .
\end{aligned}
$$

Since $I_{\iota \sigma}$ is well defined over $E_{\mathfrak{p}}$, up to numbers in $E_{\mathfrak{p}}, c_{H T, \sigma, L}^{ \pm}(\varphi)$ is equal to the determinant of the isomorphism $I_{i_{p} \sigma}$, and the representation $V_{\mathfrak{\beta}}^{ \pm}$and $H_{\mathfrak{p}}(M) / V_{\mathfrak{\beta}}^{ \pm}$are of Hodge-Tate type. Thus we have the $p$-adic periods in $\Omega$ when $M$ satisfies $\left(\operatorname{Red}_{ \pm}\right)$, because the periods $c_{H T, \sigma, \iota}^{ \pm}(\varphi) \in B_{H T}$ is, up to the degree 0 number $\operatorname{det}\left(I_{\llcorner\sigma}\right)$ in $E_{\mathfrak{p}}$, the determinant of the comparison map between $\bigwedge^{d^{ \pm}} H_{D R}^{ \pm}(M)$ and the HodgeTate representation $\bigwedge^{d^{ \pm}}\left(H_{\mathfrak{p}}(M) / V_{\mathfrak{B}}^{\mp}\right)$ and hence is supported by a single graded component. We can similarly check that $c_{H T, \sigma, \iota}^{ \pm}(\varphi)$ is supported by a single graded component also for complex place $\sigma$. Hereafter, we write $c_{p, \sigma, \iota}^{ \pm}(\varphi)$ and $c_{p, \iota}^{ \pm}(\varphi)$ for $c_{H T, \sigma, \iota}^{ \pm}(\varphi)$ and $c_{H T, \iota}^{ \pm}(\varphi)$ regarded as being in $\Omega$. The above argument shows the principal transcendental factor comes from non-vanishing $\operatorname{det}\left(I_{i_{p} \sigma}\right)$. The vanishing may occur due to the first factor $I_{\iota \sigma}$.

\subsection{An example of vanishing $p$-adic periods}

Y. André [1] found an example of vanishing period, and his argument generalizes in the following case. We keep the notation in Section 3.2. Let $\mathscr{E}$ be an elliptic curve defined over $\mathbb{Q}$ without complex multiplication. We consider the $p$-adic Tate module $T_{p}(\mathscr{E})$ of $\mathscr{E}$. Choosing a base of the dual $\left(T_{p}(\mathscr{E})\right)^{\vee} \hookrightarrow H_{p}^{1}(\mathscr{E})$, we have a representation $\varphi: \mathscr{G}_{\mathbb{Q}} \rightarrow G L_{2}\left(\mathbb{Z}_{p}\right)$. We suppose

(Fu) The image of $\varphi$ is full, that is, $\operatorname{Im}(\varphi) \supset S L_{2}\left(\mathbb{Z}_{p}\right)$;

(Ord) $\mathscr{E}$ has ordinary good reduction at $p$.

There is a plenty of examples of $\mathscr{E}$ satisfying the above conditions. We pick two embeddings $\iota: \overline{\mathbb{Q}} \hookrightarrow \mathbb{C}$ and $i_{p}: \overline{\mathbb{Q}} \hookrightarrow \Omega$. Then by (Ord), $H_{p}^{1}(\mathscr{E})$ has a one dimensional 
subspace $V_{+}=V_{-}$stable under the decomposition group at $p$ relative to $i_{p}$. Since $c$ has two eigenvalues \pm 1 on $H_{p}^{1}(\mathscr{E})$, we can choose a matrix $A \in S L_{2}\left(\mathbb{Z}_{p}\right)$ so that $A^{-1} \varphi(c) A$ leaves $V_{+}$stable and acts on it through multiplication by \pm 1 . We choose $\tau \in \mathscr{G}_{\mathbb{Q}}$ such that $\varphi(\tau)=A$. Then the complex conjugation with respect to $\iota \tau$ leaves $V_{+}$stable and acts on it through multiplication by \pm 1 , and it is clear that $c_{p, \iota \tau}^{ \pm}(\varphi)=0$. This argument shows that on a non-empty open subset of the space of $\left\{\left(i_{p}, \iota\right)\right\}$, the motive $H^{1}(\mathscr{E})$ has non-vanishing periods but also on a non-empty closed subset, the elliptic curve $\mathscr{E}$ has $p$-adic periods $c_{p, \iota \tau}^{+}(\varphi) c_{p, \iota \tau}^{-}(\varphi)=0$.

\subsection{Non-vanishing of $p$-adic periods}

Suppose $\left(\operatorname{Red}_{ \pm}\right)$for $M$ and that $M_{/ F}$ is pure of rank $n$ with coefficients in $E$ and $H_{\mathfrak{p}}(M)$ is of Hodge-Tate type at every $\mathfrak{R} \in S_{F}$. We write the representation of $\mathscr{G}_{F}$ on $H_{\mathfrak{p}}(M)$ as $\varphi$. We identify $\mathscr{G}_{F}$ with $\mathscr{G}_{F^{\sigma}}$ by $\sigma$. Then we put $\varphi_{\sigma}(g)=\varphi\left(\sigma g \sigma^{-1}\right)$ as a representation of $\mathscr{G}_{F^{\sigma}}$. Then we assume

$\varphi_{\sigma}$ is semi-simple for all $\sigma \in I$.

As we have already remarked, $\left(\operatorname{Red}_{ \pm}\right)$does not depends on $F$. We also know that $\prod_{\sigma} c_{p, \sigma, \iota \tau}^{ \pm}(\varphi)$ is equal to $c_{p, \mathrm{id}, \iota \tau}^{ \pm}\left(\operatorname{Ind}_{\mathscr{G}_{F}}^{\varphi_{Q}} \varphi\right)$ up to a non-zero algebraic constant. Thus we only need to prove the non-vanishing of $c_{p, \text { id, } \iota \tau}^{ \pm}(\Phi)$ for some $\tau$ for $\Phi=\operatorname{Ind}_{\mathscr{\varphi}_{F}}^{\mathscr{\varphi}_{Q}} \varphi$. We write $c_{p, \iota \tau}^{ \pm}$for $c_{p, \text { id }, \iota \tau}^{ \pm}$. Note that $\Phi \cong \bigoplus_{\sigma \in I} \varphi_{\sigma}$ over the Galois closure of $F$ over $\mathbb{Q}$, and $\Phi$ is semi-simple. Write $V$ for the representation space of $\Phi$, and consider the algebraic group $G L_{V}$ defined over $E_{\mathfrak{p}}$ such that $G L_{V}(A)$ is made of $A$-linear automorphisms of $V \otimes_{E_{\mathfrak{p}}} A$. Let $G$ be the Zariski closure in $G L_{V}\left(\bar{E}_{\mathfrak{p}}\right)$ of the image of $\Phi$ (for an algebraic closure $\bar{E}_{\mathfrak{p}}$ of $E_{\mathfrak{p}}$ ). Then $G$ is an algebraic subgroup of $G L_{V}$ defined over $E_{\mathfrak{p}}$, and let $G^{\circ}$ be its connected component. Since the unipotent radical $R_{u}\left(G^{\circ}\right)$ acts on $V$ by an unipotent action, the semi-simplicity of $V$ tells us that $R_{u}\left(G^{\circ}\right)$ is trivial, that is, $G^{\circ}$ and $G$ are reductive.

Now we consider the Tannakian subcategory $\mathcal{M}(M)$ of motives generated by the motive $\operatorname{Res}_{F / \mathbb{Q}} M$. We write $\operatorname{Gal}_{M}$ for the motivic Galois group of $\mathcal{M}(M)$ with respect to the fiber functor $H_{B}$. Since $\mathcal{M}(M)$ is semi-simple, $\mathrm{Gal}_{M}$ is reductive. Thus $\mathrm{Gal}_{M}$ is an algebraic group acting on $V$ defined over $E$. We have a natural morphism of algebraic groups of $G$ to $\mathrm{Gal}_{M}$ defined over $E_{\mathfrak{p}}$ induced by the functor associating to each object of $\mathcal{M}(M)$ its Galois representation. The functor taking objects of $\mathcal{M}(M)$ to their Hodge structures induces a one parameter subgroup $\mu_{\infty}: \mathbf{G}_{m / \mathbb{C}} \rightarrow \mathrm{Gal}_{M / \mathbb{C}}$ (cf. [16] Section 3), and similarly the $p$-adic Hodge-Tate structure gives another $\mu_{p}: \mathbf{G}_{m / \Omega} \rightarrow \mathrm{Gal}_{M / \Omega}$ [62]. We fix an embedding of $E_{\mathfrak{p}}$ into $\mathbb{C}$ which coincides with 
$\iota$ on $E$. Then we suppose

$$
\mu_{\infty} \text { has values in } G^{\circ}(\mathbb{C}) \text {; }
$$

$\operatorname{Res}_{F / \mathbb{Q}} M$ is regular over $F$,

that is, the image of $\mu_{\infty}$ is a regular 1-parameter subgroup of $\mathrm{Gal}_{M}$, which is equivalent to asserting that all non-trivial Hodge component of $H_{D R}(M) \otimes_{F \otimes E, \sigma \otimes \iota} \mathbb{C}$ for each $\sigma$ is of dimension 1. Note that the one parameter subgroup $\mu_{p}$ has values in $G^{\circ}(\Omega)$ by a result of Sen and Serre [62] Théorème 2. By (Reg), the centralizer of $\mu_{p}$ (resp. $\mu_{\infty}$ ) in $G$ is a maximal torus $T_{p}$ split over $\Omega$ (resp. $T_{\infty}$ split over $\mathbb{C}$ ) of $G^{\circ}$, and $\mu_{p}$ (resp. $\left.\mu_{\infty}\right)$ determines a Weyl chamber of $Y\left(T_{p}\right)=\operatorname{Hom}_{\text {alg-gr }}\left(\mathbf{G}_{m}, T_{p}\right)$ (resp. $\left.Y\left(T_{\infty}\right)\right)$ and hence a Borel subgroup $B_{p}\left(\operatorname{resp} . B_{\infty}\right)$ containing $T_{p}\left(\operatorname{resp} . T_{\infty}\right)$ in $G$. The Borel subgroups are stabilizer of the filtration coming from the Hodge filtration of $H_{D R}(M)$ through the comparison isomorphisms. Thus taking a big algebraically closed field $U$ over $E_{\mathfrak{p}}$ containing $\Omega$ and $\mathbb{C}$ as two extensions of $E_{\mathfrak{p}}$, we find $g \in G^{\circ}(U)$ such that $g T_{\infty} g^{-1}=T_{p}$ and $g B_{\infty} g^{-1}=B_{p}$. Since $\Phi(c)$ reverses the Hodge filtration on $H_{D R}(M), \Phi(c)$ brings $B_{\infty}$ to its opposite. In particular, $\Phi(c)$ (resp. $g \Phi(c) g^{-1}$ ) is not contained in any proper parabolic subgroup containig $T_{\infty}$ (resp. $T_{p}$ ). Let $\operatorname{Par}\left(T_{p}\right)$ be the set of proper parabolic subgroups of $G$ containing $T_{p}$, and define a subset $X$ of $G$ by

$$
\left\{x \in G \mid x \Phi(c) x^{-1} \text { is in an element of } \bigcup_{P \in \operatorname{Par}\left(T_{p}\right)} P\right\} .
$$

Each $P \in \operatorname{Par}\left(T_{p}\right)$ contains a unique Borel subgroup with torus $T_{p}$. There is finitely many such Borel subgroups. For a given Borel subgroup, there is a finitely many parabolic subgroups containing the Borel subgroup. Thus the set $\operatorname{Par}\left(T_{p}\right)$ is a finite set. For each $P \in \operatorname{Par}\left(T_{p}\right),\left\{x \in G \mid x \Phi(c) x^{-1} \in P\right\}$ is obviously Zariskiclosed. Then $X$ is a union of finitely many such sets and is Zariski-closed in $G$, and $\operatorname{codim}_{G^{\circ}}\left(X \cap G^{\circ}\right)>0$ because $g \notin X, g \in G^{\circ}$ and $G^{\circ}$ is connected. Since $\operatorname{Im}(\Phi)$ is Zariski-dense in $G$, we can find $\tau \in \mathscr{G}_{\mathbb{Q}}$ such that $\Phi\left(\tau^{-1}\right) \notin X$, and $\Phi\left(\tau^{-1} c \tau\right)$ is not contained in any member of $\operatorname{Par}\left(T_{p}\right)$. This shows the non-vanishing of $c_{p, \iota \tau}^{ \pm}(\Phi)$.

Theorem 3.4.1 - Suppose (Red $\left.d_{ \pm}\right),\left(S S_{F}\right)$, (MT) and (Reg) for the motive $M$ over a number field $F$. Then there exists $\tau \in \mathscr{G}_{F}$ such that $c_{p, \sigma, \iota \tau}^{ \pm}(\varphi) \neq 0$ for all $\sigma \in I$.

If we assume the Tate-style conjecture asserting that $\mathcal{M}(M)$ is equivalent to the Tannakian category of Galois representations generated by $\Phi$, then $G \cong \mathrm{Gal}_{M}$ over $E_{\mathfrak{p}}$, and hence the condition (MT) follows. If $M$ is of rank 1 , there is nothing to prove (see (7.2.1)). We assume that $M$ is of rank 2 and $M$ satisfies in addition to $\left(\operatorname{Red}_{ \pm}\right)$:

$\left(\operatorname{Irr}_{F}\right) \varphi$ is absolutely irreducible over any finite extension $F^{\prime} / F$. 
Let $V$ be the representation space of $\varphi$. We consider the Zariski closure $H$ of the image of $\varphi_{\sigma}$ in $G L_{V}\left(\bar{E}_{\mathfrak{p}}\right)$ for $\sigma$. Note that $H$ is independent of $\sigma$. The Hodge structure of $M$ at $\iota \sigma$ (resp. the $p$-adic Hodge-Tate structure at $i_{p} \sigma$ ) induces a one parameter subgroup $\mu_{\infty, \sigma}: \mathbf{G}_{m} \rightarrow \mathrm{Gal}_{M}$ (resp. $\mu_{p, \sigma}: \mathbf{G}_{m} \rightarrow H$ ). By $\left(\operatorname{Irr}_{F}\right)$, for real $\sigma, H$ is isomorphic to either $S L(2)$ or $G L(2)$ according as the weight of the Hodge structure at $\sigma$ of $M \wedge M$ is zero or not. By making some Tate twist if necessary, we may assume that $H_{\sigma}=G L(2)$. Then $\mu_{\infty, \sigma}$ has values in $H(\mathbb{C})$ (this is an $F$-version of the condition (MT)). We now assume a version of the condition (Reg):

$\left(\operatorname{Reg}_{\sigma}\right) H_{B}\left({ }^{\sigma} M\right)$ has two distinct Hodge types at each real $\sigma \in I$ and $\iota \circ \iota_{E}$, and $H_{B}\left({ }^{\sigma} M\right) \oplus H_{B}\left({ }^{c \sigma} M\right)$ has four distinct Hodge types at complex $\sigma \in I$ and $\iota \circ \iota_{E}$.

By this, we have the maximal torus $T_{X, \sigma}$ centralizing $\mu_{X, \sigma}$ and a Borel subgroup $B_{X, \sigma}$ of $H$ fixing the Hodge filtration. We take a universal domain $U$ as in the proof of Theorem 3.4.1. We fix a Borel subgroup $B$ of $H$ defined over $U$. For each real place $\sigma$ of $F$, we can choose $g_{\sigma} \in H^{\circ}(U)$ so that $g_{\sigma} T_{\infty, \sigma} g_{\sigma}^{-1}=T_{p, \sigma}$ and $g_{\sigma} B_{\infty, \sigma} g_{\sigma}^{-1}=B_{p, \sigma}$, where $H^{\circ}$ is the connected component of $H$. Then $g_{\sigma}\left(\varphi_{\sigma}\right)(c) g_{\sigma}^{-1}$ is not contained in $B_{p, \sigma}$. Thus $X_{\sigma}=\left\{x \in H \mid x\left(\varphi_{\sigma}\right)(c) x^{-1} \in \bigcup_{P \in \operatorname{Par}\left(T_{p, \sigma}\right)} P\right\}$ is a proper Zariski-closed subset of $H$, where $\operatorname{Par}\left(T_{p, \sigma}\right)$ is the set of all proper parabolic subgroups of $H$ containing $T_{p, \sigma}$. In particular, $\operatorname{codim}_{H^{\circ}}\left(X_{\sigma} \bigcap H^{\circ}\right)$ is positive. We now assume $\sigma$ to be a complex embedding of $F$. If one of $V_{\mathfrak{B}}^{ \pm}$and $V_{\mathfrak{\beta} c}^{ \pm}$ is trivial (i.e. just $\{0\}$ ) for the $p$-adic place $\mathfrak{R}$ attached to $i_{p} \sigma$, the period $c_{X, \sigma, \iota}^{ \pm}(M)$ does not vanish for any choice of $\iota$ by the definition of $H_{B}^{ \pm}\left({ }^{\iota \sigma} M\right)$. Thus we may assume that $\operatorname{dim}\left(V_{\mathfrak{B}}^{ \pm}\right)=\operatorname{dim}\left(V_{\mathfrak{B} c}^{ \pm}\right)=1$. In this case, if one of $c_{X, \sigma, \iota}^{ \pm}(M)$ vanishes, the complex conjugation $c: H_{B}\left({ }^{\iota \sigma} M\right) \cong H_{B}\left({ }^{c \iota \sigma} M\right)$ induces an isomorphism: $V_{\mathfrak{\beta}}^{ \pm} \cong V_{\mathfrak{\beta} c}^{ \pm}$. This implies the $p$-adic Hodge types of the two $\mathscr{G}_{F_{\mathfrak{\beta}}}$-modules are the same, which contradicts to $\left(\operatorname{Reg}_{\sigma}\right)$. Thus under $\left(\operatorname{Reg}_{\sigma}\right)$, the period does not vanish for complex $\sigma$. Thus we can forget complex places. Regard naturally $G^{\circ}$ in the proof of Theorem 3.4.1 as a subgroup of the product $H^{I}=\prod_{\sigma} H_{\sigma}$ of $I$-copies of $H_{\sigma}=H$. Since $\operatorname{codim}_{H^{\circ}}\left(X_{\sigma} \bigcap H^{\circ}\right)>0$, we have $\tau \in \mathscr{G}_{F}$ such that $\varphi_{\sigma}(\tau) \notin X_{\sigma}$ but $\Phi(\tau) \in G^{\circ}$. Thus $\Phi(\tau)=\bigoplus_{\sigma} \varphi_{\sigma}(\tau) \notin X_{\sigma} \times H^{I-\{\sigma\}}$; hence the codimension of $G^{\circ} \bigcap X_{\sigma} \times H^{I-\{\sigma\}}$ in $G^{\circ}$ is positive, and $G^{\circ} \bigcap\left(\bigcup_{\sigma: r e a l} X_{\sigma} \times H^{I-\{\sigma\}}\right)$ is a proper Zariski-closed subset of $G^{\circ}$. Thus we can find $\tau \in \mathscr{G}_{F}$ such that $\Phi(\tau) \notin\left(\bigcup_{\sigma: r e a l} X_{\sigma} \times H^{I-\{\sigma\}}\right)$. By changing $\iota$ by $\iota \tau$, we have a non-vanishing $p$-adic period for every $\sigma \in I$. We just record the above fact:

Corollary 3.4.2 - Suppose that $M$ is of rank 2 over $E$ and (Red $\left.{ }_{ \pm}\right)$, (Reg $\left.{ }_{\sigma}\right)$ for all $\sigma$ and $\left(\operatorname{Irr}_{F}\right)$. Then there exists $\tau \in \mathscr{G}_{F}$ such that $c_{p, \sigma, \iota \tau}^{+}(\varphi) c_{p, \sigma, \iota \tau}^{-}(\varphi) \neq 0$ for all $\sigma \in I$. 


\subsection{Admissibility condition}

Our condition $\left(\operatorname{Red}_{ \pm}\right)$is closely related to Panchishkin's admissibility condition for the (conjectural) existence of bounded $p$-adic measures for motivic $L$-functions (Panchishkin [56] Section 5). Here we explain the relation in some detail. For simplicity, we assume $F$ to be totally real. To define the admissibility, we need to introduce the following extra hypotheses on $M$ :

(Cris) $H_{\ell}(M)$ (resp. $\left.H_{p}(M)\right)$ is unramified for $\ell \neq p$ (resp. crystalline at each $\mathfrak{B} \mid p$ ).

Let us recall the definition that $M$ is crystalline at $\mathfrak{P}[22]$ : Let $B_{\text {cris }} \subset B_{D R}$ be the Fontaine's ring $B_{\text {cris }}$ introduced in [22] Section 4 , and $B_{c r i s}$ is an algebra over the field $K_{0} \subset \Omega$ of fractions of the ring of Witt vectors with coefficients in $\overline{\mathbb{F}}_{p}$. The Frobenius automorphism $\phi$ of $K_{0}$ extends to $B_{c r i s}$, and $B_{c r i s}$ has a $\phi$-linear map $\Phi_{\text {cris }}$ (called crystalline Frobenius map) and a decreasing filtration $F^{i} B_{\text {cris }}$. Then $B_{H T}$ is defined by $g r\left(B_{c r i s}\right)=\operatorname{gr}\left(B_{D R}\right)$, where the filtration of $B_{D R}$ is given by the power of the maximal ideal of $B_{D R}^{+}$, and that of $B_{c r i s}$ is induced by the filtration of $B_{D R}$. Let $K / \mathbb{Q}_{p}$ be a finite extension, $\bar{K}$ be the algebraic closure of $K$ in $\Omega$ and $K_{u r}=K_{0} \bigcap K$. Then $\mathscr{G}=\operatorname{Gal}(\bar{K} / K)$ acts on $B_{D R}$ and $B_{\text {cris }}$ extending its action on $\bar{K}$ so that $H^{0}\left(\mathscr{G}, B_{D R}\right)=K, H^{0}\left(\mathscr{G}, B_{H T}\right)=K$ and $H^{0}\left(\mathscr{G}, B_{\text {cris }}\right)=K_{\text {ur }}$. For each continuous representation of $\mathscr{G}$ on a finite dimensional vector space $V$ over $\mathbb{Q}_{p}$, we define

$$
\begin{array}{r}
D(V)=H^{0}\left(\mathscr{G}, V \otimes_{\mathbb{Q}_{p}} B_{\text {cris }}\right), \quad D_{D R}(V)=H^{0}\left(\mathscr{G}, V \otimes_{\mathbb{Q}_{p}} B_{D R}\right) \text { and } \\
D_{H T}(V)=H^{0}\left(\mathscr{G}, V \otimes_{\mathbb{Q}_{p}} B_{H T}\right),
\end{array}
$$

where $\sigma \in \mathscr{G}$ acts on $V \otimes B_{X}(X=$ cris, $D R$ or $H T)$ by $v \otimes b \mapsto \sigma(v) \otimes \sigma(b)$. Then $D(V) \otimes_{K_{u r}} K$ is isomorphic to $H^{0}\left(\mathscr{G}, V \otimes_{\mathbb{Q}_{p}}\left(B_{c r i s} \otimes_{K_{u r}} K\right)\right)$ and is a subspace of $D_{D R}(V)$. It has been shown in [23] Section 3 and [22] Section 5 that

(Fo3) $D(V) \otimes_{K_{u r}} K$ has decreasing filtration $D^{i}$ induced from that of $B_{c r i s} \otimes_{K_{u r}} K$,

$$
D(V) \text { has } \phi \text {-linear automorphism } \Phi \text { induced by } \Phi_{\text {cris }} \text {. }
$$

When the equality: $\operatorname{dim}_{K_{u r}} D(V)=\operatorname{dim}_{\mathbb{Q}_{p}} V$ holds, $V$ is called crystalline. A filtered Dieudonné module $D$ is a finite dimensional $K_{u r}$-vector space $D$ with a $\phi$ linear map $\Phi$ and a decreasing filtration $D^{i}$ of $D \otimes_{K_{u r}} K$ satisfying the condition (Fo3-4) for $D$ in place of $D(V)$. We call $M$ crystalline at $p$ if the $p$-adic étale realization $H_{p}(M)$ is crystalline for the closure $K=F_{\Re}$ of $i_{p}(\sigma(F))$ in $\Omega$ for all $\sigma \in I$. Thus we have the above functor $D$ depending on $p$-adic place $\mathfrak{B}$ induced by $i_{p} \sigma$ of $F$ which gives the identification of the decomposition group at $\mathfrak{B}$ and $\mathscr{G}$. Under 
this setting, $M$ has its crystalline realization $H_{\text {cris, } \mathfrak{\beta}}(M)$ at $\mathfrak{R}$ given by $D\left(H_{p}(M)\right)$, which is a $K_{u r}$-vector space with a $\phi$-linear automorphism $\Phi$. We suppose to have a canonical identification: $H_{c r i s, \mathfrak{\beta}}(M) \otimes_{K_{u r}} K \cong H_{D R}(M) \otimes_{F, \sigma} K$ and to have a canonical isomorphism:

$\left(\mathrm{C}_{\text {cris }}\right) \quad I_{i_{p} \sigma, \text { cris }}: H_{p}(M) \otimes_{\mathbb{Q}_{p}} B_{\text {cris }} \cong H_{\text {cris }, \mathfrak{B}}(M) \otimes_{K_{\text {ur }}} B_{\text {cris }}$

If $M$ is the motive attached to a smooth projective variety $V$ over $F$ with good reduction at $\mathfrak{B}$, by a theorem of Faltings [20] (see also [50]), $M$ is crystalline at $p$, and $H_{c r i s, \mathfrak{\beta}}(M)$ is the scalar extension to $K_{u r}$ of the crystalline cohomology group of the reduction of $V$ modulo $\mathfrak{P}$, and (Cris) and $\left(C_{\text {cris }}\right)$ are satisfied. We can take $\mathscr{C}_{X}$ to be the category of crystalline motives defined in [2]. We define the period $c_{c r i s, \sigma}^{ \pm}(\varphi)$ taking $X=B_{c r i s}$ and $I_{X, \sigma}=I_{i_{p} \sigma, c r i s} \circ I_{\iota \sigma, e t}$. We assume here the compatibility of $I_{i_{p} \sigma, c r i s}$ and $I_{i_{p} \sigma, D R}$ (see [50] 4.3.5 and [2] 5.1). Then for crystalline $M$, we get $c_{c r i s, \sigma}^{ \pm}(\varphi)=c_{D R, \sigma}^{ \pm}(\varphi)$ in $B_{D R}$. The local Euler factor at $\mathfrak{B}$ of $L(s, M)$ : $L_{\mathfrak{B}}(X)=\operatorname{det}\left(1-\left.\varphi\left(\phi^{f}\right)\right|_{H_{\mathrm{I}}(M)} X\right)$, where $f=\left[K_{u r}: \mathbb{Q}_{p}\right]$, can be recovered as the characteristic polynomial of $\Phi^{f}$ acting on $H_{\text {cris, },(}(M)$. For each filtered Dieudonné module $D$ with Frobenius $\Phi$, we can attach two polygons. One is the Newton polygon $P_{N}(D)$ of $\operatorname{det}\left(1-\left.\Phi^{f}\right|_{D} X\right)$, and the other is the Hodge polygon coming from the filtration. That is, the polygon $P_{H}(D)(t)$ passing through points

$$
(0,0), \ldots,\left(\sum_{i \leq j} \operatorname{dim}\left(D^{i} / D^{i+1}\right), \sum_{i \leq j} i \cdot \operatorname{dim}\left(D^{i} / D^{i+1}\right)\right), \ldots
$$

If $V$ is crystalline, it is known that $P_{N}(D)$ is located above $P_{H}(D)$, and they match at the two extremes [23] Section 4.4. A filtered Dieudonné module with this property is called weakly admissible. The functor $V \mapsto D(V)$ gives an equivalence of categories from the category of crystalline representations to the category of admissible filtered Dieudonné modules ([23] Section 3). Fontaine proved that admissible Dieudonné module is weakly admissible.

Let us now describe the $\mathfrak{P}$-admissibility condition of $M$ due to Panchishkin: Let $P_{N, \mathfrak{R}}(t)$ (resp. $P_{H, \mathfrak{N}}(t)$ ) be the Newton (resp. Hodge) polygon for $H_{c r i s, \mathfrak{*}}(M)$. We write $d^{+}$for $\operatorname{dim}_{\mathbb{Q}} H_{B}^{+}\left({ }^{\sigma} M\right)=\operatorname{dim}_{F} \mathscr{F}^{-}(M)$. The motive $M$ is called admissible in $[56]$ if

$$
P_{H, \mathfrak{N}}\left(d^{+}\right)=P_{N, \mathfrak{R}}\left(d^{+}\right) \quad \text { for all } \mathfrak{B}
$$

Since the Newton polygon $P_{N, \Re}$ is defined solely using the crystalline realization, if $M$ is admissible, we can find a filtered Dieudonné submodule $D_{\mathfrak{\beta}}^{-}$of $H_{\text {cris, }, \mathfrak{\beta}}(M)$ such that $D_{\mathfrak{B}}^{-} \otimes_{K_{u r}} B_{D R}=\mathscr{F}^{-}(M) \otimes_{F, i_{p} \sigma} B_{D R}$. Thus if $M$ is admissible, $D_{\mathfrak{P}}^{-}$ is weakly admissible and is contained in the admissible module $H_{c r i s, \mathfrak{B}}(M)$. In 
this situation, $D_{\Re}^{-}$itself is admissible [23] 4.5.1. Thus we can find a crystalline subrepresentation $V_{\Re}^{-} \subset H_{p}(M)$ of $\mathscr{G}_{F_{\mathfrak{\beta}}}$ such that $D\left(V_{\Re}^{-}\right)=D_{\mathfrak{\beta}}^{-}$, and $I_{i_{p} \sigma, \text { cris }}$ induces $V_{\mathfrak{B}}^{-} \otimes_{\mathbb{Q}_{p}} B_{H T} \cong \mathscr{F}^{-}(M) \otimes_{F, i_{p} \sigma} B_{H T}$.

In order to define Euler $p$-factor of the genuine $p$-adic $L$-function, we recall here some of definition given in [56] Section 6. Since the Euler $p$-factor is the same for $M$ and $\operatorname{Res}_{F / \mathbb{Q}} M$, we assume here that $F=\mathbb{Q}$. Let $\mathbb{Q}_{\infty}$ be the unique cyclotomic $\mathbb{Z}_{p}$-extension of $\mathbb{Q}$, and pick a finite order character $\chi$ of $\operatorname{Gal}\left(\mathbb{Q}_{\infty} / \mathbb{Q}\right)$. We now write

$$
L_{p}(X)=\prod_{j}\left(1-\alpha_{j} X\right)
$$

so that $\operatorname{ord}_{p} \alpha_{1} \leq \operatorname{ord}_{p} \alpha_{2} \leq \cdots \leq \operatorname{ord}_{p} \alpha_{n}$. Then we define

(E)

$$
\begin{aligned}
& \mathscr{E}(M \otimes \chi ; s) \\
& = \begin{cases}\prod_{d^{+}<j \leq n}\left(1-\alpha_{j} p^{-s}\right) \prod_{1 \leq j \leq d^{+}}\left(1-\alpha_{j}^{-1} p^{s-1}\right) & \text { if } \chi \text { is trivial, } \\
\prod_{1 \leq j \leq d^{+}}\left(\alpha_{j}^{-1} p^{s}\right)^{\nu} & \text { if } C(\chi)=p^{\nu} \text { with } \nu>0,\end{cases}
\end{aligned}
$$

where $C(\chi)$ is the conductor of $\chi$. We further put

(Г) $\quad \gamma(M)=\frac{\Gamma(1, M)}{(2 \pi i)^{r}}$ for $r=\sum_{j<0} j h^{j, k}$ for the Hodge number $h^{j, k}$ of $\check{M}$,

where $\Gamma(s, M)$ is the Gamma factor of the $L$-function of $M$ as in [15]. 


\section{Periods of arithmetic Galois representations}

We study general D-adic arithmetic Galois representations $\varphi$ and its periods. We shall state conjectures on the existence of genuine $p$-adic $L$-functions and their normalization.

\subsection{Variation of $p$-adic periods over $\operatorname{Spec}(\mathbb{Q})$}

We study a bit more general representation $\varphi: \mathscr{G}_{F} \rightarrow G L_{n}(\mathbb{K})$ than in the introduction. We assume

(Cont) There is an घ-module of finite type $V$ in $\mathbb{K}^{n}$ such that

$$
V \otimes_{\mathbb{1}} \mathbb{K}=\mathbb{K}^{n}
$$

$V$ is stable under $\varphi$;

(iii) $\varphi: \mathscr{G}_{F} \rightarrow A u t_{\rrbracket}(V)$ is continuous under the adic topology of the maximal ideal of $\llbracket$.

Replacing $V$ by its reflexive closure (that is, the intersection of localizations of

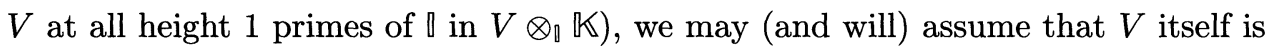
reflexive. See [8] Chapter 7 for general theory of reflexive modules. By extending the scalar field $K$ if necessary, we assume that in $\operatorname{Spec}(\mathbb{Q})(K)$, there are densely populated arithmetic points. We also assume unramifiedness (unr) as in the introduction. For an arithmetic point $P$, we consider the following condition

$\left(\operatorname{Fr}_{P}\right)$ The localization $V_{P}$ of $V$ at $P$ is free of rank $n$ over $\rrbracket_{P}$.

This condition is open, i.e., it is satisfied by all $P$ 's in a non-empty Zariski-open subset of $\operatorname{Spec}(\mathbb{\mathbb { Q }})$. Under $\left(\operatorname{Fr}_{P}\right)$, we have the residual representation $V\left(\varphi_{P}\right)$ given by the image of $V$ in $V_{P} / P V_{P}$, which is a continuous Galois representation into $G L_{n}(\mathcal{O})$. We call $P$ motivic if $\varphi_{P}$ is associated to a rank $n$ pure motive $M_{P}$ defined over $F$ with coefficients in a number field $E_{P}$ via the inclusion $i_{p}: \overline{\mathbb{Q}} \rightarrow \Omega$. We suppose arithmeticity:

(A1) Motivic points are densely populated in Spec(ロ)(K). 
We then consider the following condition milder than $\left(\mathrm{A} 2_{ \pm}\right)$: $\left(\mathrm{A}^{\prime} 2_{ \pm}\right)$For a dense subset of motivic points $P$, we have

(i) The Tate twist $M_{P}(1)$ is critical in the sense of Deligne [15], and $H_{\mathfrak{p}}\left(M_{P}\right)$ is of Hodge-Tate type at every $\mathfrak{B} \in S_{F}$;

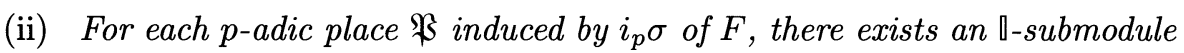
$V_{\mathfrak{\beta}}^{\mp} \subset V$ stable under $\mathscr{G}_{F_{\mathfrak{B}}}$ such that the comparison isomorphism $I_{\sigma, H T}$ induces: $V_{\mathfrak{B}}^{ \pm} \otimes_{\mathbb{Q}, P} B_{H T} \cong \mathscr{F}^{ \pm}\left(M_{P}\right) \otimes_{F \otimes E, i_{p} \sigma \otimes i_{p}} B_{H T}$.

We may assume that $V_{\mathfrak{B}}^{ \pm} \otimes_{\mathbb{1}} \mathbb{K} \cap V=V_{\mathfrak{B}}^{ \pm}$and $V_{\mathfrak{B}}^{ \pm}$is reflexive. Suppose $\left(\mathrm{A}^{\prime} 2_{ \pm}\right)$ and

$\left(\mathrm{NV}_{ \pm}\right)$There exists an embedding $i_{\infty}: \overline{\mathbb{Q}} \hookrightarrow \mathbb{C}$ such that $c_{p, i_{\infty}}^{ \pm}\left(\varphi_{P}\right) \neq 0$ for at least one motivic $P$ as in $\left(A^{\prime} 2_{ \pm}\right)$.

We want to prove $c_{p, i_{\infty}}^{+}\left(\varphi_{P}\right) \neq 0$ for densely populated $P$ satisfying $\left(\mathrm{A}^{\prime} 2_{+}\right)$. By replacing $\varphi$ by $\operatorname{Ind}_{\mathscr{G}_{F}}^{\mathscr{G}_{\mathbb{Q}}} \varphi$ (and $M$ by $\left.\operatorname{Res}_{F / \mathbb{Q}} M\right)$, we may assume that $F=\mathbb{Q}$, because the condition $\left(\mathrm{A}^{\prime} 2_{ \pm}\right)$is insensitive to induction process as shown in Section 3.2 of Chapter 3. Writing $V[c \pm 1]$ for the F-eigenspace of $c$ of the complex conjugation induced by $i_{\infty}$, we have

$$
V \otimes_{\mathbb{Z}} \mathbb{Q}=\left(V[c-1] \otimes_{\mathbb{Z}} \mathbb{Q}\right) \bigoplus\left(V[c+1] \otimes_{\mathbb{Z}} \mathbb{Q}\right)
$$

Thus we have a natural linear map $I_{1}: V[c-1] \otimes_{\mathbb{Z}} \mathbb{Q} \rightarrow\left(V / V_{p}^{-}\right) \otimes_{\mathbb{Z}} \mathbb{Q}$. We now localize at $P$. The condition $\left(\mathrm{NV}_{+}\right)$implies that, after reducing modulo $P$, $I_{1}$ induces an isomorphism. Thus the cokernel $\operatorname{Coker}\left(I_{1}\right)$ is a torsion $\mathbb{\otimes _ { \mathbb { Z } }} \mathbb{Q}$ module, and $V[c-1] \cap V_{p}^{-}=\{0\}$. Let $X=X\left(i_{\infty}\right)$ be the support of $\operatorname{Coker}\left(I_{1}\right)$ in $\operatorname{Spec}\left(\square \otimes_{\mathbb{Z}} \mathbb{Q}\right)$, which is a proper Zariski-closed subset of $\operatorname{Spec}\left(\square \otimes_{\mathbb{Z}} \mathbb{Q}\right)$. Thus for critical motivic points $P$ outside $X, c_{p, i_{\infty}}^{+}\left(\varphi_{P}\right) \neq 0$. Although we only discussed here about $c_{p, i_{\infty}}^{+}\left(\varphi_{P}\right)$, the same argument works well for $c_{p, i_{\infty}}^{-}\left(\varphi_{P}\right)$ under $\left(\mathrm{A}^{\prime} 2_{-}\right)$. Thus we find

Proposition 4.1.1 - Suppose $\left(N V_{ \pm}\right)$. Then $c_{p, i_{\infty}}^{ \pm}\left(\varphi_{P}\right) \neq 0$ for densely populated motivic points $P$ satisfying $\left(A^{\prime} 2_{ \pm}\right)$for a given $\left\{V_{\mathfrak{B}}^{\mp}\right\}_{P \in S_{F}}$.

Now suppose that $c_{p, i_{\infty} \tau}^{ \pm}\left(\varphi_{Q}\right) \neq 0$ for $\tau \in \mathscr{G}_{\mathbb{Q}}$ with another motivic point $Q$ as in $\left(\mathrm{A}^{\prime} 2_{ \pm}\right)$. We localize 』 into $\rrbracket^{\prime}$ so that we have an open immersion $\operatorname{Spec}\left(\mathbb{\square}^{\prime}\right) \hookrightarrow$

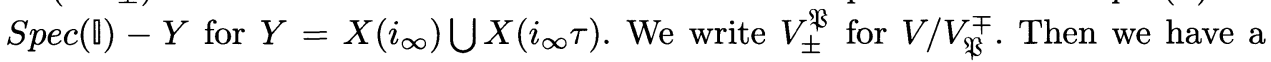
diagram:

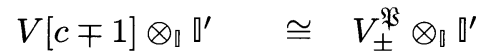

$$
\begin{aligned}
& \varphi(\tau)^{-1} \downarrow \quad \downarrow \Phi_{ \pm}(\tau)
\end{aligned}
$$

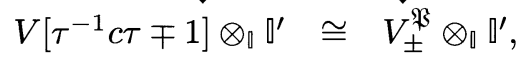


where $\Phi_{ \pm}(\tau)$ is defined so that the diagram becomes commutative. Then the following diagram for each motivic point $P$ outside $Y$ satisfying $\left(\mathrm{A}^{\prime} 2_{+}\right)$is commutative:

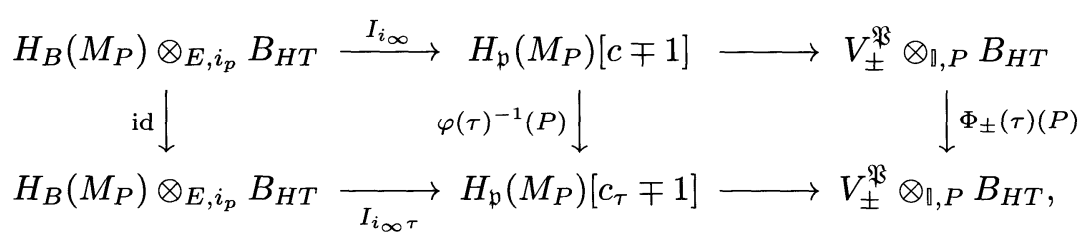

where $c_{\tau}=\tau^{-1} c \tau$. Note that $V_{ \pm}^{\mathfrak{B}} \otimes_{\natural, P} B_{H T} \cong H_{D R}^{ \pm}\left(M_{P}\right) \otimes_{F \otimes E, i_{p} \otimes i_{p}} B_{H T}$. Then we have $c_{p, i_{\infty} \tau}^{ \pm}\left(\varphi_{P}\right)=c_{p, i_{\infty}}^{ \pm}\left(\varphi_{P}\right) \beta_{ \pm}(P)$ for $\beta_{ \pm}(\tau)=\operatorname{det}\left(\Phi_{ \pm}(\tau)\right) \in \mathbb{\mathbb { d }}^{\prime}-\{0\}$. Here we regard $\beta$ as a function on $\mathscr{Q}_{\mathbb{1}}=\operatorname{Spec}(\mathbb{Q})$ by $\beta(P)=P(\beta)$. Writing the characteristic

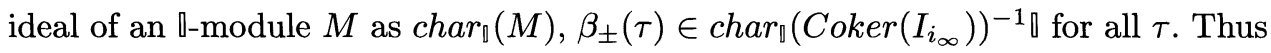
we have

Proposition 4.1.2 - Suppose $\left(A^{\prime} 2_{ \pm}\right)$. Then if $c_{p, i_{\infty}}^{ \pm}\left(\varphi_{P}\right) c_{p, i_{\infty} \tau}^{ \pm}\left(\varphi_{Q}\right) \neq 0$ for two motivic points $P$ and $Q$ satisfying $\left(A^{\prime} 2_{ \pm}\right)$, then there exists an element $\beta_{ \pm}=$ $\beta_{ \pm}(\tau) \in \mathbb{K}^{\times}$such that $c_{p, i_{\infty} \tau}^{ \pm}\left(\varphi_{P}\right)=c_{p, i_{\infty}}^{ \pm}\left(\varphi_{P}\right) \beta_{ \pm}(P)$ for densely populated motivic $P$ satisfying $\left(A^{\prime} 2_{ \pm}\right)$. Moreover the $\mathbb{\square}$-module generated by $\beta_{ \pm}(\tau)$ for all $\tau \in \mathscr{G}_{\mathbb{Q}}$ is a

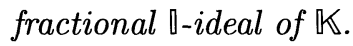

\subsection{Genuine $p$-adic $L$-functions}

Let $\varphi$ be an arithmetic -adic Galois representation satisfying (Cont), (A1,3), $\left(\mathrm{A}^{\prime} 2_{-}\right),\left(\mathrm{NV}_{-}\right)$and (unr). We also write $\mathscr{X}_{\mathbb{1}}$ for $\operatorname{Spec}(\mathbb{\square})$. Thus for an $\mathbb{O}$-algebra $A$, $\mathscr{X}_{\mathbb{1}}(A)=\operatorname{Hom}_{\mathcal{O}-a l g}(\mathbb{Q}, A)$. We state here a refined version of a conjecture in [56] Conjecture $\mathrm{II}^{\prime}$ :

Conjecture 4.2.1 - Under (Cont), (A1), (A'2-), (A3), (NV-) and (unr), there exists a genuine $p$-adic L-function for $\varphi$ and $S=\left\{V_{\mathfrak{B}}^{+}\right\}_{P \in S_{F}}$, which is a meromorphic function $L_{S, i_{\infty}}(P, \varphi)$ defined on $\mathscr{X}_{\mathbb{1}}(\Omega)$ such that for all motivic points $P$ satisfying $\left(A^{\prime} 2_{-}\right)$for $S$, if $c_{p, i_{\infty}}^{+}\left(\varphi_{P} \otimes \mathcal{N}^{-1}\right) \neq 0$,

$$
\frac{L_{S, i_{\infty}}(P, \varphi)}{c_{p, i_{\infty}}^{+}\left(\varphi_{P} \otimes \mathcal{N}^{-1}\right)} \in i_{p}(\overline{\mathbb{Q}})
$$

$$
i_{p}^{-1}\left(\frac{L_{S, i_{\infty}}(P, \varphi)}{c_{p, i_{\infty}}^{+}\left(\varphi_{P} \otimes \mathcal{N}^{-1}\right)}\right)=* \mathscr{E}(P) i_{\infty}^{-1}\left(\frac{L_{i_{\infty}}\left(1, \varphi_{P}\right)}{c_{\infty, i_{\infty}}^{+}\left(\varphi_{P} \otimes \mathcal{N}-1\right)}\right),
$$
where $\mathcal{N}$ is the cyclotomic character such that $\mathcal{N}\left(\phi_{\mathfrak{l}}\right)=N(\mathfrak{l})$;

(iii) If $M_{P}=M \otimes \chi$ for a finite order character $\chi$ factoring through the cyclotomic $\mathbb{Z}_{p}$-extension of $F$ for a motive $M$ crystalline at $p$ in the sense of [2], we have the following description of “*” and $\mathscr{E}(P):$ Replacing $M$ and 
$M_{P}$ by their restriction of scalar to $\mathbb{Q}$, we may assume that $F=\mathbb{Q}$. Then

$$
\begin{aligned}
\mathscr{E}(P) & =\mathscr{E}(M \otimes \chi, 1) \quad \text { for } \mathscr{E} \text { in }(E) \text { in Section } 3.5 \text { in Chapter 3; and } \\
* & =\gamma(M) \varepsilon(M \otimes \chi)^{-1} \quad \text { for } \gamma \text { in }(\Gamma) \text { in Section 3.5 of Chapter 3, }
\end{aligned}
$$

where $\varepsilon(M)$ is the constant term of the functional equation of $L_{i_{\infty}}(s, M)$.

Here are several remarks:

(a) In Conjecture 4.2.1 (iii), the formula of the constant " $*$ " looks a bit different from [56] Section 6 because our evaluation point is " 1 " in place of " 0 ". By the functional equation, the value $L(1, M \otimes \chi)$ corresponds to $L\left(0, \check{M} \otimes \chi^{-1}\right)$. If one applies the formula of Coates and Perrin-Riou to this dual $\check{M} \otimes \chi^{-1}$, we get the same value as in [56] Section 6. This shift of evaluation point comes from the fact that these authors use homological formulation (and the arithmetic Frobenius) instead of the cohomological one (and the geometric Frobenius) used here. Thus the Selmer group corresponding to our $p$-adic $L$ is actually the one associated to $\check{\varphi}$ defined in [28]. If crystalline motivic points $P$ of a given type is dense in $\operatorname{Spec}(\mathbb{})$, the condition (iii) uniquely determines the $p$-adic $L$ function, and the restriction of our $p$-adic $L$ to the cyclotomic deformation of $M_{P}$ should be closely related to the $p$-adic $L$-functions of $M_{P}$ conjectured in [56] and [58] (see Section 4.3). The density of crystalline motivic points seems to hold in many examples of modular Galois representations $\varphi$ (see Chapters 6,7 and 8 ). In the examples of $p$-adic $L$-functions in the modular case, the constant " $*$ " is determined completely for all motivic critical points of given type, and the expression of "*" confirms the formula (iii) in the conjecture for crystalline $P$.

(b) In the conjecture, we mean by a meromorphic function an element in the field of fractions of $\square \widehat{\otimes}_{0} \mathrm{O}_{\Omega}$.

(c) The identity (ii) is independent of the choice of basis of $H_{D R}^{+}\left(M_{P}(1)\right)$ and $H_{B}^{+}\left({ }^{i_{\infty} \sigma} M_{P}(1)\right)$ because $c_{p}^{+}$and $c_{\infty}^{+}$behave exactly in the same way under the change of basis (see (BC) in Section 3.1 of Chapter 3 ). If we change $i_{\infty}$ by $i_{\infty} \tau$ with non-vanishing periods, we know the existence of $\beta_{-} \in \mathbb{K}^{\times}$such that $c_{p, i_{\infty} \tau}^{+}\left(\varphi_{P} \otimes \mathcal{N}^{-1}\right)=\beta_{-}(P) c_{p, i_{\infty}}^{+}\left(\varphi_{P} \otimes \mathcal{N}^{-1}\right)$. Thus if the genuine $p$-adic $L$ function $L_{S, i_{\infty}}(\varphi)$ exists for $i_{\infty}$, then it is easy to deduce from the conjecture of Deligne (D) (in Section 3.1 of Chapter 3) that $\beta_{-}(\tau) L_{S, i_{\infty}}(\varphi)$ satisfies the properties (i) and (ii) for $i_{\infty} \tau$ in place of $i_{\infty}$. Thus the existence of $L_{S, i_{\infty}}(\varphi)$ is independent of the choice of $i_{\infty}$.

\subsection{Normalized $p$-adic $L$-functions}

We assume $p>2$ and the following conditions for an arithmetic $\varphi$ : 
$\left(\mathrm{Fr}_{\mathbb{q}}\right) \varphi$ has values in $G L_{n}(\mathbb{\square})$;

$\left(\mathrm{FR}_{ \pm}\right) V_{\mathfrak{B}}^{ \pm}$is an $\llbracket$-direct summand of the space $V=V(\varphi) \cong \square^{n}$ for all $\mathfrak{B}$;

(SD) There is a Galois character $\chi: \mathscr{G}_{F} \rightarrow \mathbb{1}^{\times}$such that $\check{\varphi} \otimes \chi \cong \varphi$ over $\mathbb{K}$.

By self-duality (SD), the character $\chi$ is arithmetic, and the isomorphism takes $V_{\mathfrak{B}}^{ \pm}(\check{\varphi} \otimes \chi) \otimes \mathbb{K}$ into $V_{\mathfrak{B}}^{ \pm}(\varphi) \otimes \mathbb{K}$. Since each pure motive has a polarization, the condition (SD) should not be too restrictive. Any 2-dimensional representations and their tensor products satisfy (SD). We thus have an inner product $\langle$,$\rangle on V(\varphi)$ such that

$$
\langle\varphi(\sigma) x, \varphi(\sigma) y\rangle=\chi(\sigma)\langle x, y\rangle \text {. }
$$

We define the normalizing factor $\alpha_{ \pm}(\varphi) \in \mathbb{Q}$. Let $\check{V}$ be the dual of $V$ in $V \otimes \mathbb{K}$ under the pairing. Then $\check{V}$ is Galois stable and belongs to isomorphism class of $\check{\varphi} \otimes \chi$ over ๆ. Although it is more legitimate to write $V\left((\check{\varphi} \otimes \chi)_{P}\right)$ for $\check{V} / P \check{V}$, we write it as $\check{V}\left(\varphi_{P}\right)$. If the place $\mathfrak{P}$ is induced by $i_{p} \sigma$, we write $\check{V}_{\sigma}^{ \pm}$for $\check{V}_{\mathfrak{B}}^{ \pm}$. The -freeness of $W_{ \pm}=\check{V}_{\sigma}^{ \pm} \bigoplus \check{V}_{c \sigma}^{ \pm}+(\check{V} \bigoplus \check{V})\left[c_{\sigma} \pm 1\right]$ for complex $i_{\infty} \sigma$ and $W_{ \pm}=\check{V}_{\sigma}^{ \pm}+\check{V}\left[c_{\sigma} \pm 1\right]$ for real $i_{\infty} \sigma$ follows from $\left(N V_{ \pm}\right)$, and we find an 0 -linear endomorphism $A_{\sigma}^{ \pm}$such that $A_{\sigma}^{ \pm} \check{V}=W_{ \pm}$or $A_{\sigma}^{ \pm}(\check{V} \bigoplus \check{V})=W_{ \pm}$according as $i_{\infty} \sigma$ is real or complex. We put $\alpha_{ \pm}=a_{ \pm}(\varphi)=\prod_{\sigma \in \Sigma} \alpha_{\sigma}^{ \pm}$for $\alpha_{\sigma}^{ \pm}=\operatorname{det}\left(A_{\sigma}^{ \pm}\right)$computed with respect to a basis of $\check{V}$ or $\check{V} \bigoplus \check{V}$. By definition, $\alpha_{ \pm}(\varphi)$, up to $\llbracket$-units, only depends on the isomorphism class of $\check{\varphi}$ and hence depends only on the isomorphism class of $\varphi$, but not on the choice of the pairing. We define

(NL) $L_{S}(\varphi)=\alpha_{+}(\varphi)^{-1} L_{S, i_{\infty}}(\varphi)=\alpha_{+}(\varphi)^{-1} L_{S, i_{\infty}}(\check{\varphi} \otimes \chi)$,

where the last equality follows from the fact that $\varphi \cong \check{\varphi} \otimes \chi$ over $\mathbb{K}$. Since $\check{V}_{\mathfrak{B}}^{-}\left(\varphi \otimes \mathcal{N}^{-1}\right)=\check{V}_{\mathfrak{P}}^{+}(\varphi)$ and $\check{V} / \check{W}_{+} \cong\left(\check{V} / \check{V}_{\mathfrak{\beta}}^{+}\right) / \check{V}\left[c_{\sigma}+1\right]$, it is clear from the argument proving Proposition 4.1.2 that the ideal $\left(L_{S}(\varphi)\right)$ of $\llbracket \widehat{\otimes}_{\mathcal{O}} \sigma_{\Omega}$ depends only on isomorphism class of $\varphi$ but not on the choice of $i_{\infty}$. We will give another proof of this fact later.

We want to relate now the values of the normalized $L$-function $L_{S}(\varphi)$ with the complex $L$-values. For notational simplicity, we assume that $F=\mathbb{Q}$ for this, and we leave the reader the task of working out the formula for general $F$. For an arithmetic point $P$, classically the complex period $c_{\infty, i_{\infty}}^{ \pm}\left(M_{P}\right)$ is normalized choosing a suitable lattice $L_{\text {? }}$ of various realizations $H_{?}\left(M_{P}\right)$. Since the $L$-function would describe the Selmer group of $\check{\varphi}$ (see [28]), it would be natural to take $\check{V} / P \check{V}$ as $L_{\mathfrak{p}}$ of $H_{\mathfrak{p}}\left(M_{P}\right)$. As for $L_{B}$, writing $\mathscr{V}$ for the valuation ring of the place $i_{p}$ of $E$, we would choose an $\mathscr{V}$-free lattice $L_{B}$ of $H_{B}\left(M_{P}\right)$ so that $I_{i_{\infty}}$ induces an isomorphism:

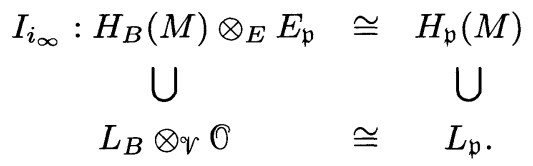


Since Faltings' comparison map preserves well the integral structure on $H_{\mathfrak{p}}(M)$ and $H_{D R}(M)$ when $M$ is associated to a smooth projective variety over $\mathbb{Q}$ with a smooth model over $\mathbb{Z}_{p}$ ([20] Theorem 5.3 and [21] (c) in the introduction), it seems possible to impose a condition similar to $(\infty)$ to normalize $L_{D R}$ under certain assumption on $M$. However we do not specify here how to normalize the $\mathscr{V}$-free lattice $L_{D R}$ in $H_{D R}(M)$, because of the following reasons:

(i) the choice of $L_{D R}$ does not really affect the final expression (see Proposition 4.3.1),

(ii) the normalization of $L_{D R}$ could be delicate depending on $M$ especially if $M$ is not the total cohomology of a smooth variety over $\mathbb{Z}_{p}$, and

(iii) it would add further complication to the text. Instead we will give later an example, in the case where $M$ is associated to an elliptic curve, to clarify the point.

Since the Tate motive $\mathbb{Q}(1)$ has canonical integral structure $\mathbb{Z}(1)$ whose $p$-adic period is 1 and complex period is $2 \pi i$. By tensoring $\mathbb{Z}(1)^{\otimes m}$, we can shift $L_{\text {? }}$ to the integral structure $L_{?}(m)$ in $H_{?}(M(m))$. We then define the complex period $\Omega_{\infty}^{ \pm}\left(\varphi_{P} \otimes\right.$ $\left.\mathcal{N}^{-m}\right)$ (resp. the $p$-adic period $\left.\Omega_{p}^{ \pm}\left(\varphi_{P} \otimes \mathcal{N}^{-m}\right)\right)$ by the $i_{\infty}$-component of $\operatorname{det}\left(I_{\mathbb{C}}\right)(\operatorname{resp}$. the $i_{p}$-component of $\left.\operatorname{det}\left(I_{i_{p}}\right)\right)$ computed with respect to the basis of $L_{B}(m)[c \mp 1]$ $\left(\right.$ resp. $\left.L_{\mathfrak{p}}(m) /\left(\check{V}_{p}^{\mp}\left(\varphi_{P} \otimes \mathcal{N}^{-m}\right)\right)\right)$ and $L_{D R}(m) /\left(L_{D R}(m) \cap \mathscr{F}^{\mp}\left(M_{P}(m)\right)\right)$. Now we have

Proposition 4.3.1 - Let the notation be as above. We assume $(S D),\left(F r_{0}\right),\left(F R_{+}\right)$ and $\left(N V_{+}\right)$. Then we have

$$
\frac{L_{S}(P, \varphi)}{\Omega_{p}^{+}\left(\varphi_{P} \otimes \mathcal{N}^{-1}\right)}=* i_{p} i_{\infty}^{-1}\left(\frac{L_{i_{\infty}}\left(1, \varphi_{P}\right)}{\Omega_{\infty}^{+}\left(\varphi_{P} \otimes \mathcal{N}^{-1}\right)}\right)
$$

up to $p$-adic units.

Proof. Note that $\check{V}_{p}^{-}\left(\varphi \otimes \mathcal{N}^{-1}\right)=\check{V}_{p}^{+}(\varphi)$ and $\check{V}\left(\varphi \otimes \mathcal{N}^{-1}\right)[c-1]=\check{V}(\varphi)[c+1]$. Thus $\alpha_{-}\left(\varphi \otimes \mathcal{N}^{-1}\right)=\alpha_{+}(\varphi)$. Then, by $(\infty), \alpha_{+}(P)=\operatorname{det}\left(I_{i_{\infty}}: L_{B}[c+1] \rightarrow\right.$ $\left.L_{\mathfrak{p}} /\left(\check{V}_{p}^{+} / P \check{V}_{p}^{+}\right)\right)$up to $p$-adic units. Then we know that $c_{p, i_{\infty}}^{+}\left(\varphi_{P} \otimes \mathcal{N}^{-1}\right)$, computed with respect to $L_{D R}(1)$ and $L_{B}(1)$, is equal to $\left.\alpha_{+}(P) \Omega_{p}^{+}\left(\varphi_{P} \otimes \mathcal{N}^{-1}\right)\right)$ up to a $p$-adic unit. This combined with the interpolation formula (Int) in Chapter 1 for $L_{S, i_{\infty}}(\varphi)$ yields the result.

Since the periods $\Omega_{p}^{ \pm}$does not depends on $i_{\infty}$, Proposition 4.3.1 shows the independence of the ideal $\left(L_{S}(\varphi)\right)$ from $i_{\infty}$. Further, if $L_{D R}$ is chosen so that $\left.\Omega_{p}^{+}\left(\varphi_{P} \otimes \mathcal{N}^{-m}\right)\right)$ is a $p$-adic unit, then $L_{S}(P, \varphi)$ is equal to the normalized complex $L$-value $* \frac{L_{i_{\infty}}\left(1, \varphi_{P}\right)}{\Omega_{\infty}^{+}\left(\varphi_{P} \otimes \mathcal{N}^{-1}\right)}$ up to units. Suppose that $Q$ is another motivic point such that $\varphi_{Q}=\varphi_{P} \otimes \psi$ for a character $\psi$. Then $\psi(-1)=1$. Since we have a natural 
integral structure on the motive $M(\psi)$ of $\psi$, similarly to the case where $\psi$ is the cyclotomic character, we can shift the integral structure given by $L_{\text {? }}$ or even a basis of $L_{?}$ on $M_{P}$ to $M_{Q}$, which again satisfies $(\infty)$. Thus, we have well defined period $\Omega_{\infty}^{+}\left(\varphi_{P} \otimes \psi \mathcal{N}^{-1}\right)$ of $M_{Q}$ for arithmetic points $Q$ (with $\varphi_{Q}=\varphi_{P} \otimes \psi$ ) on the cyclotomic deformation locus in $\operatorname{Spec}(\mathbb{Q})$. On this locus,

$$
\frac{L_{S}(\varphi)}{\Omega_{p}^{+}\left(\varphi_{P} \otimes \mathcal{N}^{-1}\right)} \quad \text { interpolates } \quad * i_{p} i_{\infty}^{-1}\left(\frac{L_{i_{\infty}}\left(1, \varphi_{P} \otimes \psi\right)}{\Omega_{\infty}^{+}\left(\varphi_{P} \otimes \psi \mathcal{N}^{-1}\right)}\right) \quad \text { for variable } \psi
$$

This argument combined with (iii) of Conjecture 4.2.1 shows that the restriction of $\frac{L_{S}(\varphi)}{\Omega_{p}^{+}\left(\varphi_{P} \otimes \mathcal{N}^{-1}\right)}$ to the cyclotomic line passing through $P$ is equal to the cyclotomic $p$-adic $L$-function interpolating the complex $L$-values normalized with respect to $\check{V}$.

We extend the definition of the normalized period $\Omega_{p}^{ \pm}$for a general base field $F$. We put $L_{\mathfrak{p}}=\check{V} / P \check{V}$ and for each $\sigma$, we choose an $\mathfrak{r}_{E}$-free lattice $L_{B, \sigma}$ of $H_{B}\left({ }^{\sigma} M\right)$ so that the comparison isomorphism induces $L_{B, \sigma} \otimes 0 \cong L_{\mathfrak{p}}$. We also choose an $\mathfrak{r}_{F} \otimes_{\mathbb{Z}} \mathfrak{r}_{E}$-free lattice $L_{D R}$ of $H_{D R}(M)$. Then we define $\Omega_{p, \sigma}^{ \pm}\left(\varphi_{P} \otimes\right.$ $\left.\mathcal{N}^{-m}\right)$ by the determinant of $I_{i_{p}}$ from $L_{\mathfrak{p}}(m) /\left(L_{\mathfrak{p}}(m) \bigcap \check{V}_{\sigma}^{\mp}\left(\varphi_{P} \otimes \mathcal{N}^{-m}\right)\right)$ ) into $L_{D R}(m) /\left(L_{D R}(m) \bigcap_{\mathscr{F}^{\mp}}\left(M_{P}(m)\right)\right)$. Then we have

$(\mathrm{NP}) \quad c_{p, \sigma, i_{\infty}}^{ \pm}\left(\varphi_{P} \otimes \mathcal{N}^{-m}\right)=\alpha_{\sigma}^{ \pm(-1)^{m+1}}(P) \Omega_{p, \sigma}^{ \pm}\left(\varphi_{P} \otimes \mathcal{N}^{-m}\right) \quad$ if $i_{\infty} \sigma$ is real, $c_{p, \sigma, i_{\infty}}^{ \pm}\left(\varphi_{P} \otimes \mathcal{N}^{-m}\right)=\alpha_{\sigma}^{ \pm(-1)^{m+1}}(P) \Omega_{p, \sigma}^{ \pm}\left(\varphi_{P} \otimes \mathcal{N}^{-m}\right) \Omega_{p, c \sigma}^{ \pm}\left(\varphi_{P} \otimes \mathcal{N}^{-m}\right) \quad$ otherwise,

where the left-hand side is also computed with respect to $L_{B}(m)$ and $L_{D R}(m)$.

In formulating a main conjecture in the Iwasawa theory relating the characteristic ideal of the Selmer group of $\check{\varphi}$ with an analytic $p$-adic $L$-function, I expect the $L$ function $L_{S}(\varphi)$ to play an important role, although we might have to further modify the $p$-adic $L$-function in residually reducible cases (that is, the case where $\varphi \bmod \mathfrak{m}_{\square}$ is not absolutely irreducible). When $\check{V}[c+1] \cong \check{V} / \check{V}_{p}^{+}$(that is, $\alpha_{+}$is a unit), the ideal $\left(L_{S, i_{\infty}}(\varphi)\right)$ and $\left(L_{S}(\varphi)\right)$ coincide, we do not need to modify $L_{S, i_{\infty}}(\varphi)$ and have the precise interpolation property (Int). Thus it is natural to ask when $V[c \pm 1] \cong V / V_{p}^{ \pm}$ holds. Here we list an obviously sufficient condition in the simplest case where $F=\mathbb{Q}$ and $n=2$ : Write $\bar{\varphi}$ for the representation on $V(\bar{\varphi})=V(\varphi) / \mathfrak{m}_{\mathbb{1}} V(\varphi)$ induced by $\varphi$ for the maximal ideal $\mathfrak{m}_{\mathbb{v}}$ of $\mathbb{\|}$. We assume that

(Sp) $V=V_{p}^{+} \bigoplus V^{\prime}$ for a complement $V^{\prime} \neq 0$, and $V=V[c-1] \bigoplus V[c+1]$.

We leave the proof of the following fact to the reader.

Proposition 4.3.2 - Suppose $n=2, F=\mathbb{Q}$ and (Sp). If $\bar{\varphi}$ is irreducible, then there exists a complex embedding $i_{\infty}$ such that $\check{V}[c \pm 1] \cong \check{V} / \check{V}_{p}^{ \pm}$.

We now assume that $M=M_{P}$ is associated with a motive given by $H^{1}$ of an elliptic curve $\mathscr{E}$ defined over $\mathbb{Q}$ with ordinary good reduction at $p$. We use the same 
symbol $\mathscr{E} / \mathbb{Z}$ for the Néron model of $\mathscr{E}$. Thus $E=\mathbb{Q}$ and $\mathfrak{p}=p$. We assume the lattices $L_{?}(m)$ of $H_{?}(M(m))$ to be equal to $H_{e t}^{1}\left(\mathscr{E}, \mathbb{Z}_{p}\right), H_{B}^{1}(\mathscr{E}, \mathbb{Z})$ and $H_{D R}^{1}(\mathscr{E}, \mathbb{Z})$. Then these lattices satisfy $(\infty)$, and we assume $L_{p}=\check{V} / P \check{V}$, which is actually self dual. Then we have the following five related exact sequences:

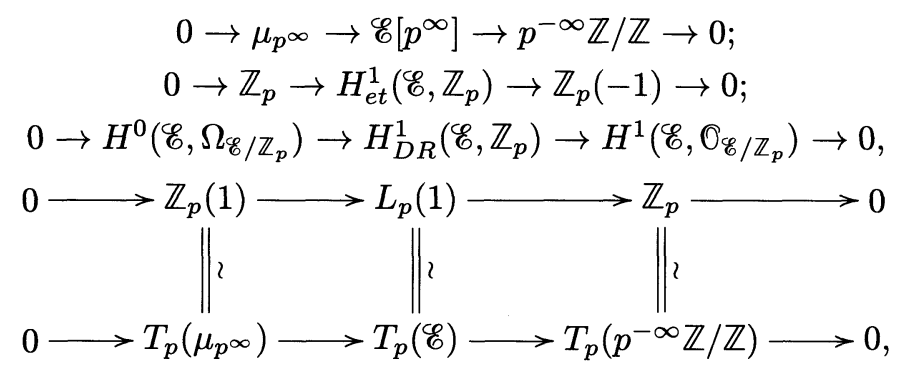

where $\mathscr{E}\left[p^{\infty}\right]$ is the $p$-divisible group of $\mathscr{E}, T_{p}(?)$ indicate the $p$-adic Tate module of the object ? and the last diagram is commutative. Note that $T_{p}(\mathscr{E})=\check{L}_{p}$ for the dual $\check{L}_{p}$ of $L_{p}$. This implies the complex period and $p$-adic period $c_{*}^{ \pm}(M(1))$ can be computed by taking the generator $\omega$ of $\mathscr{F}^{ \pm}\left(M(1)^{\vee}\right)=\mathscr{F}^{ \pm}(M)$ over $\mathbb{Q}$ and choosing a generator $b^{ \pm}$of $H_{B}^{ \pm}(\check{M})=H_{B}^{ \pm}(M(1))$, and $c_{*}^{ \pm}(M(1))=\left\langle b^{ \pm}, \omega\right\rangle$ for the duality pairing $\langle$,$\rangle between M(1)^{\vee}$ and $M(1)$. We can normalize $\omega$ taking the Néron differential on the Néron model $\mathscr{E} / \mathbb{Z}$ of $\mathscr{E}$ over $\mathbb{Z}$, that is, $H^{0}\left(\mathscr{E} / \mathbb{Z}, \Omega_{\mathscr{E} / \mathbb{Z}}\right)=\mathbb{Z} \omega$, because $\mathscr{F}^{ \pm}(M) \supset H^{0}\left(\mathscr{E}, \Omega_{E / \mathbb{Q}}\right)$. We normalize $b^{ \pm}$taking a generator of $L_{B}(1)[c \mp 1]$. Under this choice, $c_{\infty}^{ \pm}(M(1))$ is the canonical period $\Omega_{ \pm}(\mathscr{E})$ of $\mathscr{E}$. Then $\Omega_{p}^{+}(\mathscr{E})=$ $\operatorname{det}\left(I_{i_{p}}\right)=\langle e, \omega\rangle$ for a generator $e$ of $\mathbb{Z}_{p}=L_{p}(1) / \mathbb{Z}_{p}(1)$ in (E4). By [20] Theorem 5.3, if $p>2, \Omega_{p}^{+}(\mathscr{E})$ is a unit in $\mathcal{O}_{\Omega}$. Writing $I_{i_{\infty}}\left(b^{-}\right)=u(\mathscr{E}) e$, we have $u(\mathscr{E})=\alpha_{+}(P)$, and we see from (Int) that

$$
\begin{aligned}
i_{p}^{-1}\left(\frac{L_{S, i_{\infty}}(P, \varphi)}{u(E) \Omega_{p}^{+}(\mathscr{E})}\right) & =* i_{\infty}^{-1}\left(\frac{L_{i_{\infty}}\left(1, \varphi_{P}\right)}{\Omega_{+}(\mathscr{E})}\right) \text { and } \\
i_{p}^{-1}\left(\frac{L_{S}(P, \varphi)}{\Omega_{p}^{+}(\mathscr{E})}\right) & =* i_{\infty}^{-1}\left(\frac{L_{i_{\infty}}\left(1, \varphi_{P}\right)}{\Omega_{+}(\mathscr{E})}\right) .
\end{aligned}
$$

We now show that we can always choose in the isogeny class of $\mathscr{E} / \mathbb{Z}$ an elliptic curve which satisfies $L_{p}(1)[c-1] \cong L_{p}(1) / \mathbb{Z}_{p}(1)$ for some $i_{\infty}$ (that is, $u(\mathscr{E})$ is a unit). We may assume that we have chosen $i_{\infty}$ such that the $p$-adic valuation of $u(\mathscr{E})$ is minimal. If $u(\mathscr{E})$ is a unit, there is nothing to prove. Suppose not, and write $I_{i_{\infty} \sigma}\left(b^{-}\right)=u_{\sigma} e$. Thus $u_{\sigma} \equiv 0 \bmod p$ for all $\sigma$, and $\varphi(\sigma) V(\bar{\varphi})[c-1]$ coincides with $V_{p}^{-} / \mathfrak{m}_{0} V_{p}^{-}$for all $\sigma$. Thus $V(\bar{\varphi})[c-1]$ is stable under the global Galois group. We take maximal subgroup $C$ of $\mathscr{E}\left[p^{\infty}\right][c-1]$ stable under the global Galois action. Since the Galois action on the Tate module is irreducible, $C$ is a finite group. Then for $\mathscr{E}^{\prime}=\mathscr{E} / C, \mathscr{E}^{\prime}\left[p^{\infty}\right][c-1]$ does not contain subgroup stable under the global Galois action. This implies that $\mathscr{E}^{\prime}$ is what we wanted. Thus $u\left(\mathscr{E}^{\prime}\right)$ is a unit, and $\Omega_{p}^{+}\left(\mathscr{E}^{\prime}\right)$ is also a unit as already seen. 


\subsection{Singularity}

We try to locate singularity of the normalized $L_{S}(\varphi)$. We continue to assume $\left(\mathrm{Fr}_{\mathbb{0}}\right),\left(\mathrm{FR}_{ \pm}\right)$and $p>2$. A principle related to the conjectures of Artin and Tate is $\left(\mathrm{AT}_{\infty}\right)$ the complex L-function $\Gamma\left(s, \varphi_{P}\right) L\left(s, \varphi_{P}\right)=\Gamma\left(s, \varphi_{P}\right) L\left(0, \varphi_{P} \otimes \mathcal{N}^{s}\right)$ has a pole of order $r$ at $s$ implies that $s \in \mathbb{Z}$ and $\varphi_{P} \otimes \mathcal{N}^{s} \bigoplus\left(\varphi_{P} \otimes \mathcal{N}^{s}\right)^{*}$ contains the trivial Galois character with multiplicity $r$.

Thus we ask

Question 4.4.1 - If there exists a p-adic L-function $L_{S, i_{\infty}}(\varphi)$ satisfying the requirements of Conjecture 4.2.1, can one expect the following principle for the normalized $L_{S}(\varphi)$ ? For each prime divisor $\mathbf{P}$ of $\operatorname{Spec}(\mathbb{Q})$ and $\varphi^{*}=\check{\varphi} \otimes \mathcal{N}$,

$\left(\mathrm{AT}_{p}\right) L_{S}(\varphi)$ has a pole at $\mathbf{P} \Rightarrow \varphi_{\mathbf{P}} \bigoplus \varphi_{\mathbf{P}}^{*}$ has the trivial representation as a subquotient.

One might be tempted to expect, after the analysis of the Selmer group of $\varphi$ done by Greenberg [28] Section 4, a more stronger assertion

(G) the order of the pole at each prime divisor $\mathbf{P}$ is equal to

$$
\ell_{\mathbf{P}}(\varphi)=\ell_{\mathbf{P}}\left(H_{0}\left(\varphi_{F}, \varphi \bigoplus \varphi^{*}\right)\right)
$$

Here $\ell_{\mathbf{P}}(X)$ is the length of the localization at $\mathbf{P}$ of a torsion $\|$-module $X$. We say that $L_{S}(\varphi)$ satisfies $\left(\mathrm{AT}_{p}\right)$ in strict sense if $(\mathrm{G})$ holds for all prime divisors $\mathbf{P}$ of $\mathscr{X}_{\mathbb{l}}$. When $\varphi$ is residually irreducible (i.e. $\varphi \bmod \mathfrak{m}_{\mathbb{}}$ is absolutely irreducible) with dense critical crystalline points of type $S$, and $\varphi$ contains all cyclotomic deformation of its specialization, we conjecture that $L_{S}(\varphi)$ satisfies $(\mathrm{G})$ (or equivalently, $L_{S}(\varphi) \in$ $\left.\llbracket \widehat{\otimes}_{0} O_{\Omega}\right)$. However, either in the residually reducible case or in the case where $\varphi$ does not contain all cyclotomic deformation, this seems more subtle on the automorphic side: The occurrence of the trivial representation in $\varphi_{\mathbf{P}}\left(\right.$ or $\left.\varphi_{\mathbf{P}}^{*}\right)$ does not suffice to ensure the existence of a pole at $\mathbf{P}$. For example, if we take a locus $X$ in $\mathscr{X}_{0}(\Omega)$ on which $V\left(\varphi_{\mathbf{P}}\right)^{I(\Re)} \neq\{0\}$, the $L$-function $L_{X}$ interpolating the $L$-values on $X$ has generally more Euler factors at $p$ than the one $L_{\mathscr{L}}$ interpolating $L$-values all over $\mathscr{Q}_{\mathbb{1}}(\Omega)$. Thus $L_{X}$ may have a pole at a prime divisor $\mathbf{P}$ on $X$ but $L_{\mathscr{L}}$ restricted to $X$ may not have a pole at $\mathbf{P}$, that is, the pole of $L_{X}$ at $\mathbf{P}$ can be canceled by the extra-Euler factor removed from $L_{X}$. If we restrict $L_{\mathscr{L}}$ to another closed subscheme $Y$ containing $\mathbf{P}$ as a prime divisor, but generically on $Y$, we have $V\left(\varphi_{Y}\right)^{I(\mathfrak{B})}=\{0\}$, then it would be reasonable to take $L_{\mathscr{D}}$ restricted to $Y$ to be the $L$-function of $\varphi_{Y}$. Then the $L$-function does not have pole at $\mathbf{P}$ and has too less singularity at $\mathbf{P}$. Contrary to this, $\alpha_{+}(\varphi)$ can be non-unit if $\varphi_{P}$ is not semi-simple for some $\mathbf{P}$ (see Section 4.7 and Section 8.5 of Chapter 8). Since $\alpha_{+}(\varphi)(P)=\alpha_{+}(\varphi)(Q)$ if $\varphi_{P}=\varphi_{Q} \otimes \psi$ for a character $\psi$, the divisor of $\alpha_{+}(\varphi)$ contains cyclotomic variation. 
This implies that, for a prime divisor $\mathbf{P}$ in $\operatorname{div}\left(\alpha_{+}(\varphi)\right), \varphi_{\mathbf{P}}$ and $\varphi_{\mathbf{P}}^{*}$ cannot contain the trivial representation. Thus in this case, if $L_{S, i_{\infty}}(\varphi)$ does not have zero at $\mathbf{P}$, which is likely in some cases (see Section 8.5 of Chapter 8 ), $L_{S}(\varphi)$ has too much singularity. Thus what we hope in general is:

$\left(\mathrm{G}^{\prime}\right) \operatorname{ord}_{\mathbf{P}}\left(L_{S}(\varphi)\right) \leq \ell_{\mathbf{P}}(\varphi)=\ell_{\mathbf{P}}\left(H_{0}\left(\varphi_{F}, \varphi \oplus \varphi^{*}\right)\right)$

$$
\text { if } V\left(\varphi_{\mathbf{P}}\right)^{I(\Re)}=V(\varphi)^{I(\Re)} \otimes_{\mathbb{1}} \rrbracket_{\mathbf{P}} / \mathbf{P} \text { and } \alpha_{+}(\varphi) \text { is an } \rrbracket_{\mathbf{P}} \text {-unit, }
$$

where ord $\mathbf{P}$ indicates the order of pole at $\mathbf{P}$. If $L_{S}(\varphi)$ is holomorphic at $\mathbf{P}$, we just put $\operatorname{ord}_{\mathbf{P}}\left(L_{S}(\varphi)\right)=0$. We note that $\operatorname{ord}_{\mathbf{P}}\left(L_{S, i_{\infty}}(\varphi)\right)=\operatorname{ord}_{\mathbf{P}}\left(L_{S}(\varphi)\right)$ if $\alpha_{+}(\varphi)$ is an Пp-unit.

\section{5 $\mu$-invariant of cyclotomic restrictions of $L_{S}(\varphi)$}

We continue to keep the assumptions of Section 4.3 and write $\nu$ for the universal cyclotomic character, (that is, the universal deformation of the trivial character of $\operatorname{Gal}\left(\mathbb{Q}\left(\mu_{p \infty}\right) / \mathbb{Q}\right)$ into $\mathbb{Z}_{p}[[\Gamma]]^{\times}$for $\left.\Gamma=1+p \mathbb{Z}_{p}\right)$. Suppose that $\varphi$ contains all the cyclotomic variation, that is, for each point $P$ of $\mathscr{Q}_{\mathbb{l}}$, there exists a closed irreducible subscheme $\operatorname{Spec}\left(\mathbb{\square}^{P}\right)$ of $\operatorname{Spec}(\mathbb{\square})$ such that $\mathbb{\square}^{P}$ is a finite flat $\mathbb{Z}_{p}[[\Gamma]]$-algebra and

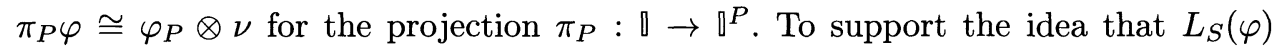
is related to the Selmer group of $\check{\varphi}$ (and $\varphi \otimes \mathcal{N}^{-1}$ ) defined in [28], we compute the variation of $\mu$-invariant of $\pi_{P}\left(L_{S}(\varphi)\right)$ over the isogeny class of $\varphi$ for a fixed point $P$ satisfying $\left(\mathrm{A} 2_{ \pm}\right)$. We let $\varphi$ act on $V(\varphi)=\rrbracket^{n}$, and take a sublattice $V\left(\varphi^{\prime}\right)$ of $V(\varphi)$ stable under $\varphi$. We compare the $\mu$-invariants of $\pi_{P}\left(L_{S}(\varphi)\right)$ and $\pi_{P}\left(L_{S}\left(\varphi^{\prime}\right)\right)$. We suppose $\left(\mathrm{Fr}_{\llbracket}\right)$ and $\left(\mathrm{FR}_{ \pm}\right)$also for $V\left(\varphi^{\prime}\right)$. Since for any character $\xi: \mathscr{G}_{F} \rightarrow \mathcal{O}^{\times}$, $P \otimes \xi\left(\alpha_{+}(\varphi)\right)=P\left(\alpha_{+}(\varphi)\right)$ up to unit in $\mathbb{0}$, we have, for the $p$-adic valuation $v$ with $v(p)=1$,

$$
\mu\left(\pi_{P}\left(L_{S}(\varphi)\right)\right)-\mu\left(\pi_{P}\left(L_{S}\left(\varphi^{\prime}\right)\right)\right)=v\left(\alpha_{+}\left(\varphi^{\prime}\right)(P)\right)-v\left(\alpha_{+}(\varphi)(P)\right) .
$$

For $\mathfrak{B}$ induced by $i_{p} \sigma$, we write

$$
\begin{aligned}
W_{\mathfrak{R}}^{ \pm}(\varphi) & = \begin{cases}\check{V}_{\mathfrak{R}}^{ \pm}(\varphi) & i_{\infty} \sigma \text { is real }, \\
\check{V}_{\mathfrak{B}}^{ \pm}(\varphi) \oplus \check{V}_{\mathfrak{B} c^{\circ}}^{ \pm}(\varphi) & i_{\infty} \sigma \text { is complex }\end{cases} \\
W(\varphi) & = \begin{cases}\check{V}^{(}(\varphi) & i_{\infty} \sigma \text { is real }, \\
\check{V}(\varphi) \oplus \check{V}(\varphi) & i_{\infty} \sigma \text { is complex. }\end{cases}
\end{aligned}
$$

Define I-linear maps $B, C_{\mathfrak{R}}^{ \pm}, D_{ \pm, \sigma}$ and $D_{\mathfrak{\Re}}^{ \pm}$so that, for $\mathfrak{R}$ induced by $i_{p} \sigma$

$$
\begin{gathered}
B\left(W\left(\varphi^{\prime}\right)\right)=W(\varphi), \quad C_{\mathfrak{\beta}}^{ \pm}\left(W_{\mathfrak{R}}^{ \pm}\left(\varphi^{\prime}\right)+W\left(\varphi^{\prime}\right)\left[c_{\sigma} \pm 1\right]\right)=W_{\mathfrak{R}}^{ \pm}(\varphi)+W(\varphi)\left[c_{\sigma} \pm 1\right], \\
D_{ \pm, \sigma}\left(W\left(\varphi^{\prime}\right)\left[c_{\sigma} \pm 1\right]\right)=W(\varphi)\left[c_{\sigma} \pm 1\right], \quad D_{\mathfrak{\beta}}^{ \pm}\left(W_{\mathfrak{R}}^{ \pm}\left(\varphi^{\prime}\right)\right)=W_{\mathfrak{B}}^{ \pm}(\varphi) .
\end{gathered}
$$


Put $\beta=\operatorname{det}(B), \gamma_{\mathfrak{\beta}}^{ \pm}=\operatorname{det}\left(C_{\mathfrak{\beta}}^{ \pm}\right), \delta_{ \pm, \sigma}=\operatorname{det}\left(D_{ \pm, \sigma}\right)$ and $\delta_{\mathfrak{B}}^{ \pm}=\operatorname{det}\left(D_{\mathfrak{B}}^{ \pm}\right)$. Then $\beta=\delta_{+, \sigma} \delta_{-, \sigma}, \gamma_{\mathfrak{\beta}}^{ \pm}=\delta_{\mathfrak{\beta}}^{ \pm} \delta_{ \pm, \sigma}$ up to units, and we have

$$
\begin{aligned}
& \alpha_{\sigma}^{ \pm}(\varphi) \alpha_{\sigma}^{ \pm}\left(\varphi^{\prime}\right)^{-1}=\beta / \gamma_{\mathfrak{P}}^{ \pm}=\delta_{\mp, \sigma} / \delta_{\mathfrak{R}}^{ \pm} \\
& \alpha_{ \pm}(\varphi) \alpha_{ \pm}\left(\varphi^{\prime}\right)^{-1}=\prod_{\sigma \in \Sigma} \delta_{\mp, \sigma} \times \prod_{\mathfrak{R}}\left(\delta_{\mathfrak{R}}^{ \pm}\right)^{-\left[F_{\mathfrak{\beta}}: \mathbb{Q}_{p}\right]}
\end{aligned}
$$

Then we get

( $\mu 1) \quad \mu\left(\pi_{P}\left(L_{S}\left(\varphi^{\prime}\right)\right)\right)-\mu\left(\pi_{P}\left(L_{S}(\varphi)\right)\right)=\sum_{\sigma \in \Sigma} v\left(\delta_{-, \sigma}(P)\right)-\sum_{\mathfrak{B}}\left[F_{\mathfrak{B}}: \mathbb{Q}_{p}\right] v\left(\delta_{\mathfrak{B}}^{+}(P)\right)$.

Writing $C$ for $\check{V}\left(\varphi^{\prime}\right) / \check{V}(\varphi)$ and $\mathscr{F}_{\mathfrak{B}}^{+} C=\check{V}_{\mathfrak{B}}^{+}\left(\varphi^{\prime}\right) / \check{V}_{\mathfrak{B}}^{+}(\varphi)$, this is equivalent to, when $0=\mathbb{Z}_{p}$

$$
\mu\left(\pi_{P}\left(L_{S}\left(\varphi^{\prime}\right)\right)\right)-\mu\left(\pi_{P}\left(L_{S}(\varphi)\right)\right)=\sum_{\sigma \in \Sigma} v\left(\#\left(C\left(F_{\sigma}\right)\right)-\sum_{\mathfrak{B}}\left[F_{\mathfrak{R}}: \mathbb{Q}_{p}\right] v\left(\#\left(\mathscr{F}_{\mathfrak{\beta}}^{+} C\right)\right),\right.
$$

where $F_{\sigma}$ is the completion of $F$ at the archimedean place $i_{\infty} \sigma$ and

$$
C\left(F_{\sigma}\right)=H_{0}\left(\operatorname{Gal}\left(\mathbb{C} / F_{\sigma}\right), C\right)
$$

This matches well with the formula of Perrin-Riou [59] of the variation of the $\mu$ invariant of the cyclotomic Selmer group of $\pi_{P} \check{\varphi}$.

\subsection{Nearly ordinary $\llbracket$-adic representations}

Here we show that the 0 -adic representation $\pi(\lambda)$ attached to a $\Lambda$-algebra homomorphism $\lambda: h^{\text {n.ord }}\left(N p^{\infty} ; \mathcal{O}\right) \rightarrow \rrbracket$ satisfies $(\mathrm{A} 1)$ and $\left(\mathrm{A}^{\prime} 2_{ \pm}\right)$. We assume that $\square$ is finite and torsion-free over $\Lambda$ (resp. $\Lambda^{\text {ord }}$ ) in the nearly ordinary case (resp. the ordinary case where $\lambda$ factors through $h^{\text {ord }}$ ). Since the condition (Gal) in Section 2.8 of Chapter 2 determines the representation $\pi(\lambda)$ only over $\mathbb{K}$, we take a representation $\varphi$ of $\mathscr{G}_{F}$ into $G L_{2}(\mathbb{Q})$ such that $\operatorname{Tr}(\varphi)=\operatorname{Tr}(\pi(\lambda))$. If there is no confusion is likely, we sometimes write $\pi(\lambda)$ for $\varphi$. For example, if $\bar{\varphi}$ is absolutely irreducible, there is no ambiguity of $\varphi$ [10]. By local class field theory, there is a canonical inclusion of groups of $F_{p}^{\times}=T_{1}\left(\mathbb{Q}_{p}\right)$ into $\prod_{\mathfrak{B} \mid p} \mathscr{G}_{F_{\mathfrak{B}}}^{a b}$ with dense image. Thus $n \in \mathbb{Z}[I]=X\left(T_{1}\right)$ gives rise to a character of $\mathscr{G}_{F_{\mathfrak{B}}}$. We write $V_{\mathfrak{B}}(n)$ for the rank one space over $\mathcal{O}$ on which $\mathscr{G}_{F_{\mathfrak{\beta}}}$ acts by a character which coincides locally with $n$ on $I_{\mathfrak{\beta}}$, where $I_{\mathfrak{\beta}}$ is the inertia subgroup of $\mathfrak{P}$ in $\mathscr{G}_{F_{\mathfrak{B}}}$. Let $P$ be a positive arithmetic point of $\mathscr{Q}_{\mathbb{1}}(\Omega)$ of weight $\kappa$. By Blasius and Rogawski [6], it is known that $V\left(\varphi_{P}\right) \otimes K$ is the étale realization $H_{\mathfrak{p}}\left(M_{P}\right)$ of a rank two motive $M_{P}$ defined over 
$F$ with coefficients in a number field $E_{P}$ unless $P$ falls in an exceptional case which is very limited (in the exceptional case, $m(\kappa)=n(\kappa)+t$ for $\left.t=\sum_{\sigma \in I} \sigma\right)$. Here $E_{P}$ is a finite extension of the field $Q\left(\lambda_{P}\right)$ generated by $P(\lambda(\mathbb{T}(\mathfrak{l})))$ for primes $\mathfrak{l}$ outside $N p$. Then as explained in Section 2.8 of Chapter 2, we have an exact sequence of $\varphi_{F_{\mathfrak{\beta}}}$-modules: $0 \rightarrow V_{\mathfrak{\beta}}(n) \rightarrow V\left(\varphi_{P}\right) \rightarrow V_{\mathfrak{\beta}}(m) \rightarrow 0$, where $m=m(\kappa)$ and $n=n(\kappa)$. Since $m>n$, a theorem of Tate tells us that $V\left(\varphi_{P}\right) \otimes K$ is of Hodge-Tate type ([68] 3.3). This fact also follows from [21] and [6] combined. Anyway we conclude from [61] Chapter III, Appendix that the Hodge-Tate type of $V\left(\varphi_{P}\right)$ is $(m, n)$ and $(n, m)$, and $V_{\mathfrak{\beta}}(n)$ plays the role of $V_{\Re}^{+}=V_{\Re}^{-}$in $\left(\operatorname{Red}_{ \pm}\right)$for $V\left(\varphi_{P}\right)$. By (iv) of (Gal) in

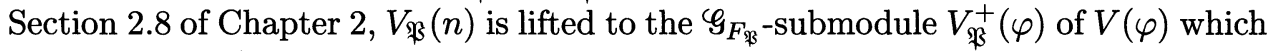
is written as $V_{\mathfrak{B}}^{+}$in (Gal). Thus $\varphi$ satisfies (A1) and $\left(\mathrm{A}^{\prime} 2_{ \pm}\right)$, and any subquotient of

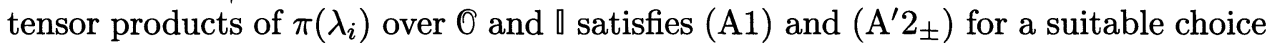
of $S$.

Let $\delta_{\mathfrak{B}}: \mathscr{G}_{F_{\mathfrak{\beta}}} \rightarrow \mathbb{q}^{\times}$be the character given in (iv) of (Gal) in Section 2.8 of Chapter 2 for $\varphi=\pi(\lambda)$, and define $\varepsilon_{\mathfrak{R}}$ by $\varepsilon_{\mathfrak{\beta}} \delta_{\mathfrak{\beta}}=\operatorname{det}(\varphi)$. Suppose that $\lambda$ is nearly ordinary. By (iv) in (Gal), the maximal closed subscheme $X$ of Spec() on which one of $\delta_{\mathfrak{N}}$ and $\varepsilon_{\mathfrak{B}}$ is trivial on $I_{\mathfrak{B}}$ for every $\mathfrak{B}$ is of codimension $[F: \mathbb{Q}]$ and is the union of the locus of $P$ for which $\varphi_{P}$ is ordinary or co-ordinary (that is, contragredient to an ordinary representation). Thus, if $[F: \mathbb{Q}]>1, \varphi_{P}$ cannot contain trivial representation as a subquotient for any prime divisor $P$ in $\operatorname{Spec}(\mathbb{\square})$. Actually the Eisenstein component of the nearly ordinary Hecke algebra is of dimension $3+2 d$ for the defect $d$ of the Leopoldt conjecture for $F$ and $p$. Thus the dimension of the locus of $P$ for which $\varphi_{P}$ or $\varphi_{P}^{*}$ is reducible (over $K$ ) is less than $2+2 d$. This gives a stronger fact that the reducible locus is of codimension at least $[F: \mathbb{Q}]-d$. When $F=\mathbb{Q}$, the ordinary locus is of codimension 1, which contains positive arithmetic point $P$ such that $\varphi_{P}$ is irreducible. Note that $\varphi^{\prime}=\operatorname{det}(\varphi)^{-1} \otimes \varphi$ is again modular, and by this operation, the co-ordinary locus of $\varphi$ is sent to the ordinary locus of $\varphi^{\prime}$. This shows the locus in $X$ on which $\varphi$ has the trivial representation as a subquotient is of codimension $\geq 2$ in $X$. Thus even if $F=\mathbb{Q}, \varphi_{\mathbf{P}}$ cannot contain trivial representation as a subquotient for any prime divisor $\mathbf{P}$ in $\operatorname{Spec}(\mathbb{\square})$. Similarly, we can show that $\varphi_{\mathbf{P}}^{*}$ cannot contain trivial representation as a subquotient for any prime divisor $\mathbf{P}$ in $\operatorname{Spec}(\mathbb{\square})$. Thus $L_{S, i_{\infty}}(\varphi)$ and $L_{S}(\varphi)$ should be both holomorphic everywhere in the nearly ordinary case of $F \neq \mathbb{Q}$.

The ordinary case is more subtle. In this case, if $\varphi_{\mathbf{P}}^{*}$ contains the trivial representation, then $\left(\varepsilon_{\mathfrak{B}}\right)_{\mathbf{P}}=\mathcal{N}$ for all $\mathfrak{P}$, because unramified $\left(\delta_{\mathfrak{B}}\right)_{\mathbf{P}}$ cannot be equal to $\mathcal{N}$. Thus $\varphi_{P}$ is of weight $k=2(\Leftrightarrow \kappa=(t, 0))$, which is arithmetic and irreducible. Thus $\varphi_{\mathbf{P}}^{*}$ never contains the trivial representation for prime divisors $\mathbf{P}$. On the other hand, $\varphi_{\mathbf{P}}$ may contain the trivial character. We can make the following conjecture: 


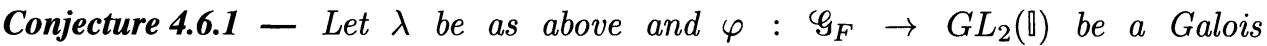
representation such that $\operatorname{Tr}(\varphi)=\operatorname{Tr}(\pi(\lambda))$. Suppose (FR $\left.R_{ \pm}\right)$for $\varphi$.

(i) (Nearly ordinary case) If gives an irreducible component of the normalization of the reduced part of $\operatorname{Spec}\left(h^{n . o r d}\left(N p^{\infty} ; \mathfrak{O}\right)\right)$, then $L_{S}(\varphi)$ and $L_{S, i_{\infty}}(\varphi)$ for $S=\left\{V_{\mathfrak{B}}^{+}(\lambda)\right\}$ are holomorphic outside prime divisors $\mathbf{P}$ with non-semi-simple $\varphi_{\mathbf{P}}$.

(ii) (Ordinary case) Suppose that $\lambda$ factors through the ordinary part, giving an irreducible component of $\operatorname{Spec}\left(h^{\text {ord }}\left(N p^{\infty} ; \mathcal{O}\right)\right)$. Then $L_{S}(\varphi)$ and $L_{S, i_{\infty}}(\varphi)$ are holomorphic outside prime divisors $\mathbf{P}$ with reducible $\varphi_{\mathbf{P}}$.

The statement (i) above tells us that the two $L$-functions are holomorphic everywhere if $F \neq \mathbb{Q}$ because we always expect $\varphi_{\mathbf{P}}$ to be irreducible in this case. We will prove (G) in Chapter 8 if $\varphi$ in (ii) is given by an induced representation of an I-adic Galois character, assuming the compatibility of comparison isomorphisms in $[50]$.

\subsection{Residually reducible case}

Here by an example, we explain what happens when $\varphi$ has non-semi-simple $\varphi_{\mathbf{P}}$. Suppose that $F=\mathbb{Q}, \pi(\lambda)$ is $p$-ordinary and unramified outside $p, \mathbb{Q}=\Lambda^{\text {ord }}$ and for a prime divisor $P=(\pi)$ of $\Lambda^{\text {ord }}$, we have an exact sequence of $\mathscr{G}_{F}$-modules:

$$
0 \rightarrow \chi \rightarrow \varphi_{P} \rightarrow \psi \rightarrow 0
$$

where $\chi$ and $\psi$ are global Galois characters acting on the rank one space $V(\chi)$, and $V(\psi)$. By ordinarity, on $V_{p}^{ \pm}$, the inertia group $I_{p}$ acts trivially. Since $\operatorname{det}(\varphi(c))=-1$, $\chi(c) \neq \psi(c)$. Since $\chi$ and $\psi$ are unramified outside $p$, one of them has to ramify at $p$, and the other is trivial by ordinarity of $\pi(\lambda)$. Thus in $\pi(\lambda)$, there are at least two isomorphism classes over $\mathbb{\mathbb { V }}$, one $\varphi$ having non-semi-simple (Eis) with $\chi$ trivial, the other $\varphi^{\prime}$ having non-semi-simple (Eis) with trivial $\psi$. First suppose that $\chi$ is trivial, then $\check{V}(\chi)=\check{V}\left(\varphi_{P}\right)[c-1]=\check{V}_{p}^{ \pm}\left(\varphi_{P}\right)$ and hence $\alpha_{+}$in (NL) of Section 4.3 is prime to $P$, and $\alpha_{-}$is divisible by $\pi$. Now we suppose that $\chi$ is ramified and $\psi$ is trivial. Since $\chi$ ramifies in this case, $\check{V}\left(\varphi_{P}^{\prime}\right) \cong \check{V}(\chi) \bigoplus \check{V}_{p}^{ \pm}\left(\varphi_{P}^{\prime}\right)$ as $\mathscr{G}_{\mathbb{Q}_{p}}$-modules, and $\check{V}\left(\varphi_{P}^{\prime}\right)[c+1]=\check{V}(\chi)$ is isomorphic to $\check{V}\left(\varphi_{P}^{\prime}\right) / \check{V}_{p}^{ \pm}\left(\varphi_{P}^{\prime}\right)$. Thus again $\alpha_{+}$is prime to $P$. In this case, if (Eis) is not split, $\alpha_{-}$can be a unit for a suitable $i_{\infty}$. Thus $\operatorname{ord}_{P}\left(L_{S}(\varphi)\right)=\operatorname{ord}_{P}\left(L_{S}\left(\varphi^{\prime}\right)\right)=\operatorname{ord}_{P}\left(L_{S, i_{\infty}}(\varphi)\right)$. Note that $\ell_{P}(\varphi)=0$ and $\ell_{P}\left(\varphi^{\prime}\right)=1$. Thus for $\varphi, L_{S}(\varphi)$ satisfies $(\mathrm{G})$ but $L_{S}\left(\varphi^{\prime}\right)$ does not. Thus the association $\phi \mapsto L_{S}(\phi)$ does not distinguish the difference of $\varphi$ and $\varphi^{\prime}$; in other words, the normalized $p$-adic $L$-functions are invariants of classes more rough than isomorphism classes (it is more strict than isogeny classes as we will give one more example of the reducible case in Section 8.5 of Chapter 8). 



\section{Periods of tensor products of motives}

We express the periods of tensor products of rank 2 motives as monomials of periods of the components. We then study tensor products of rank 2 arithmetic Galois representations and specialize Conjecture 4.2.1 in Chapter 4 to this case. There are several choices of $S=\left\{V_{\mathfrak{B}}^{+}\right\}$, and we give a classification of $p$-adic $L$-functions according to $S$. We fix an embedding $i_{\infty}$ and often drop the subscript $i_{\infty}$ from the notation.

\subsection{Tensor products of motives}

Let $E$ be a number field in $\overline{\mathbb{Q}}$. We assume $\left(\mathrm{CM}_{E}\right) \quad E$ is either a CM field or a totally real field.

Let $M$ be a pure regular motive of rank $n$ defined over $F$ with coefficients in $E$. Since every pure motive with coefficients in $\mathbb{Q}$ has a polarization [18] 6.7, the coefficient field $E$ compatible with the polarization satisfies $\left(\mathrm{CM}_{E}\right)$. We suppose

$( \pm) \mathscr{F}^{+}(M)=\mathscr{F}^{-}(M)=\mathscr{F}(M)\left(\Leftrightarrow H^{p, p}\left({ }^{\sigma} M\right)=0\right.$ for all $p$ and $\left.\sigma \in I\right)$.

This implies $n=2 m$. The field $F$ contains the largest totally real subfield $F_{+}$. If $F$ contains a $\mathrm{CM}$ field, then $F$ contains the largest $\mathrm{CM}$ subfield $F_{C M}$. For any $E \otimes_{\mathbb{Q}} F$-module $V$ and $\rho \in A u t(F / \mathbb{Q})$, we write $V^{\rho}$ for the $E \otimes F$-module $V \otimes_{F, \rho} F$. We quote the following lemma from [3] and [74] Lemma 2.1 (see [42] Lemma 1.1 for a simple proof):

Lemma 5.1.1 - (o) $H_{D R}(M)$ is $E \otimes F$-free of rank $n$;

(i) If $F$ does not contain a $C M$ field, then $\mathscr{F}(M)$ is $E \otimes F$-free of rank $m$;

(ii) Suppose that $F$ contains a $C M$ field. If the generator of $\operatorname{Gal}\left(F_{C M} / F_{+}\right)$can be extended to $\rho \in \operatorname{Aut}(F)$, then $\mathscr{F}(M) \oplus \mathscr{F}(M)^{\rho}$ is $E \otimes F$-free.

We assume that $\operatorname{rank}(M)=2$. We write $A$ (resp. $T$ ) for the subset of $\sigma \in I$ such that $\mathscr{F}(M) \otimes_{F, \sigma} \overline{\mathbb{Q}}=\{0\}\left(\right.$ resp. $\operatorname{rank}_{E \otimes \overline{\mathbb{Q}}}\left(\mathscr{F}(M) \otimes_{F, \sigma} \overline{\mathbb{Q}}\right)=1$ ). Then $I=T \bigsqcup A \bigsqcup c A$ for complex conjugation $c$, and $A \neq \emptyset$ implies that $F$ contains a CM field. For a number field $L$, we write $I_{L}$ for the set of all embeddings of $L$ into $\overline{\mathbb{Q}}$. 
Then $A$ (resp. $T$ ) is an inflated image of a subset of $I_{F_{C M}}$ (resp. $I_{F_{+}}$). We write $c_{X, J}^{ \pm}(M)=\prod_{\sigma \in J /\langle c\rangle} c_{X, \sigma}^{ \pm}(M)$ and $\delta_{X, J}(M)=\prod_{\sigma \in J} \delta_{X, \sigma}(M)$ for a subset $J$ of $I$. We have for $M$ with $\operatorname{rank}_{E} M=2$

$$
c_{X, A}^{ \pm}(M)=\delta_{X, A}(M), \quad c_{X}^{ \pm}(M) \equiv c_{X, T}^{ \pm}(M) \delta_{X, A}(M) \quad \bmod \overline{\mathbb{Q}}^{\times} .
$$

\subsection{Periods of $M_{1} \otimes M_{2}$}

Now we consider two rank 2 motives $M_{1}$ and $M_{2}$ satisfying $\left(\operatorname{Red}_{ \pm}\right)$in Section 3.2 of Chapter 3 in addition to (Reg) in Section 3.4 of Chapter 3 and $( \pm)$. We write $\left(m_{i}(\sigma), n_{i}(c \sigma)\right)$ and $\left(n_{i}(\sigma), m_{i}(c \sigma)\right)$ for the Hodge type of $M_{i}$ at $\sigma$. In this section, we study the periods for $M_{1} \otimes M_{2}$ and $A d(M)$. For each $p$-adic place $\mathfrak{P}$ associated to $i_{p} \sigma$, we write $V_{\mathfrak{B}}(M)$ for the subspace $V_{\mathfrak{R}}^{+}=V_{\mathfrak{R}}^{-}$in $H_{\mathfrak{p}}(M)$. We describe all possible cases of $V_{\Re}\left(M_{1} \otimes M_{2}\right)$, up to interchange of $M_{1}$ and $M_{2}$, in the following table as long as $M_{1} \otimes M_{2}$ satisfies $\left(\operatorname{Red}_{+}\right)$and $V_{\mathfrak{R}}\left(M_{1} \otimes M_{2}\right)$ can be described as a sum of tensor products of $H_{\mathfrak{p}}\left(M_{i}\right)$ and $V_{\mathfrak{B}}\left(M_{j}\right)$.

\begin{tabular}{|c|c|}
\hline$V_{\mathfrak{B}}\left(M_{1} \otimes M_{2}\right)$ & Conditions \\
\hline$(\mathrm{cA}) H_{\mathfrak{p}}\left(M_{1}\right) \otimes H_{\mathfrak{p}}\left(M_{2}\right)$ & $\begin{array}{l}\text { (i) } \quad \sigma \in A_{1} \cap A_{2} \\
\text { (ii) } \sigma \in A_{1} \cap T_{2}, n_{1}(\sigma)-m_{1}(c \sigma)>m_{2}(c \sigma)-n_{2}(\sigma) \\
\text { (iii) } \sigma \in A_{1} \cap c A_{2}, n_{1}(\sigma)-m_{1}(c \sigma)>m_{2}(c \sigma)-n_{2}(\sigma)\end{array}$ \\
\hline$(\mathrm{T}) V_{\mathfrak{B}}\left(M_{1}\right) \otimes H_{\mathfrak{p}}\left(M_{2}\right)$ & $\begin{array}{l}\text { (i) } \sigma \in A_{2} \cap T_{1}, n_{2}(\sigma)-m_{2}(c \sigma)>m_{1}(c \sigma)-n_{1}(\sigma) \\
\text { (ii) } \sigma \in A_{2} \cap T_{1}, m_{2}(\sigma)-n_{2}(c \sigma)<m_{1}(c \sigma)-n_{1}(\sigma) \\
\text { (iii) } \sigma \in T_{1} \cap T_{2}, m_{2}(\sigma)-n_{2}(c \sigma)<m_{1}(c \sigma)-n_{1}(\sigma) \\
\quad n_{2}(\sigma)-m_{2}(c \sigma)>n_{1}(c \sigma)-m_{1}(\sigma) \\
\text { (iv) } \sigma \in c A_{2} \cap T_{1}, n_{2}(\sigma)-m_{2}(c \sigma)>n_{1}(c \sigma)-m_{1}(\sigma)\end{array}$ \\
\hline $\begin{array}{l}(\mathrm{C}) V_{\mathfrak{R}}\left(M_{1}\right) \otimes H_{\mathfrak{p}}\left(M_{2}\right) \\
+H_{\mathfrak{p}}\left(M_{1}\right) \otimes V_{\mathfrak{\beta}}\left(M_{2}\right) \\
\end{array}$ & $\begin{array}{l}\sigma \in T_{1} \cap T_{2}, m_{1}(\sigma)-n_{1}(c \sigma)>m_{2}(c \sigma)-n_{2}(\sigma) \\
n_{1}(\sigma)-m_{1}(c \sigma)>n_{2}(c \sigma)-m_{2}(\sigma)\end{array}$ \\
\hline$(\mathrm{cC}) V_{\mathfrak{B}}\left(M_{1}\right) \otimes V_{\mathfrak{B}}\left(M_{2}\right)$ & $\begin{array}{l}\sigma \in T_{1} \cap T_{2}, m_{1}(\sigma)-n_{1}(c \sigma)<m_{2}(c \sigma)-n_{2}(\sigma) \\
n_{1}(\sigma)-m_{1}(c \sigma)<n_{2}(c \sigma)-m_{2}(\sigma)\end{array}$ \\
\hline (A) $\{0\}$ & $\begin{array}{l}\text { (i) } \sigma \in A_{1} \cap c A_{2}, m_{1}(\sigma)-n_{1}(c \sigma)<n_{2}(c \sigma)-m_{2}(\sigma) \\
\text { (ii) } \sigma \in c A_{1} \cap T_{2}, m_{1}(\sigma)-n_{1}(c \sigma)<n_{2}(c \sigma)-m_{2}(\sigma) \\
\text { (iii) } \sigma \in c A_{1} \cap c A_{2}\end{array}$ \\
\hline
\end{tabular}

In each of the above cases, one can express the periods of $M_{1} \otimes M_{2}$ as monomials of periods of $M_{1}$ and $M_{2}$. Such computation is done by Blasius and Yoshida (see [5], [74] and [42] Section 1) assuming that $X=\mathbb{C}$. Since the definition of the period is 
formal as explained in Section 3.1 of Chapter 3, the result in the papers quoted above is valid for any choice of $X$ satisfying the requirement in Section 3.1 of Chapter 3 . Here is a summary of the result obtained:

Theorem 5.2.1 - Suppose $\left(\operatorname{Red}_{+}\right)$, ( \pm ) and $\left(R e g_{\sigma}\right)$ for $M_{1}$ and $M_{2}$, and write $\delta_{\sigma}$ (resp. $c_{\sigma}^{ \pm}$) for $\delta_{X, \sigma}$ (resp. $c_{X, \sigma}^{ \pm}$). These invariants only depend on $\sigma$ modulo the subgroup generated by $c$. Then we have the following result for $M=M_{1} \otimes M_{2}$ :

(i) When $\sigma \neq c \sigma$, we have $c_{\sigma}^{+}\left(M_{i}\right)=c_{\sigma}^{-}\left(M_{i}\right)$ and

\begin{tabular}{|c|c|c|c|}
\hline Type & $\sigma \in A$ & $\sigma \in T$ & $\sigma \in C$ \\
\hline$c_{\sigma}^{+}(M)$ & $\delta_{\sigma}\left(M_{1}\right)^{2} \delta_{\sigma}\left(M_{2}\right)^{2}$ & $\delta_{\sigma}\left(M_{2}\right) c_{\sigma}^{+}\left(M_{1}\right)^{2}$ & $c_{\sigma}^{+}\left(M_{1}\right) c_{\sigma}^{+}\left(M_{2}\right) \delta_{c \sigma}\left(M_{1}\right) \delta_{c \sigma}\left(M_{2}\right)$ \\
\hline$c_{\sigma}^{-}(M)$ & $\delta_{\sigma}\left(M_{1}\right)^{2} \delta_{\sigma}\left(M_{2}\right)^{2}$ & $\delta_{\sigma}\left(M_{2}\right) c_{\sigma}^{-}\left(M_{1}\right)^{2}$ & $c_{\sigma}^{-}\left(M_{1}\right) c_{\sigma}^{-}\left(M_{2}\right) \delta_{c \sigma}\left(M_{1}\right) \delta_{c \sigma}\left(M_{2}\right)$ \\
\hline
\end{tabular}

(ii) When $\sigma=c \sigma$, only Case $T$ occurs, and we have

$$
c_{\sigma}^{ \pm}\left(M_{1} \otimes M_{2}\right)=c_{\sigma}^{+}\left(M_{1}\right) c_{\sigma}^{-}\left(M_{1}\right) \delta_{\sigma}\left(M_{2}\right)
$$

Proof. We start with a general motive $M$. First suppose that $c \sigma \neq \sigma$ for the complex conjugation $c$ induced by $i_{\infty}$. Then we have the following commutative diagram:

$$
\begin{array}{ccccc}
H_{B}^{+}\left({ }^{\sigma} M\right) & \hookrightarrow & H_{B}\left({ }^{\sigma} M\right) \oplus H_{B}\left({ }^{c \sigma} M\right) & \rightarrow & \frac{H_{D R}(M)}{\mathscr{F}(M)} \otimes_{F \otimes E,(\sigma \oplus c \sigma) \otimes \iota_{E}}(X \oplus X) \\
\alpha_{B} \| \imath & & \alpha \| 2 & \operatorname{Id} \otimes \alpha_{X} \| \imath \\
H_{B}^{-}\left({ }^{\sigma} M\right) & \hookrightarrow & H_{B}\left({ }^{\sigma} M\right) \oplus H_{B}\left({ }^{c \sigma} M\right) & \rightarrow & \frac{H_{D R}(M)}{\mathscr{F}(M)} \otimes_{F} \otimes E,(\sigma \oplus c \sigma) \otimes \iota_{E} \\
\end{array}
$$

where $\alpha\left(v \oplus v^{\prime}\right)=v \oplus\left(-v^{\prime}\right)$ for $v \oplus v^{\prime} \in H_{B}\left({ }^{\sigma} M\right) \oplus H_{B}\left({ }^{c \sigma} M\right), \alpha_{B}$ is just induced by $\alpha$, and $\alpha_{X}\left(x \oplus x^{\prime}\right)=x \oplus\left(-x^{\prime}\right)$ for $x \oplus x^{\prime} \in X \oplus X$. This shows that $c_{X, \sigma}^{+}(M)=c_{X, \sigma}^{-}(M)$. Then we get Case $T$ in (i) and (ii) from [74] Proposition 3.1. Case $C$ is studied in [42] Proposition 1.4. Case $A$ is immediate.

We now look at the rank 3 motive $A d(M)$ for a rank 2 motive $M=M_{1}$. We have a splitting: $M \otimes \check{M}=A d(M) \oplus \mathbf{1}$, where $\mathbf{1}$ is the identity motive with trivial Galois representation. Then the Hodge type of $A d(M)$ is given by $\pm(m(\sigma)-n(\sigma), n(c \sigma)-m(c \sigma))$ and $(0,0)$. Thus in this case, $\mathscr{F}^{-}(M)\left(\right.$ resp. $\left.\mathscr{F}^{+}(M)\right)$ is $E \otimes F$-free of rank 1 (resp. 2). This shows that $V_{\mathfrak{B}}^{+}(A d(M))$ is the image of the projection of $V_{\mathfrak{B}}(M) \otimes H_{\mathfrak{p}}(\check{M})+H_{\mathfrak{p}}(M) \otimes V_{\mathfrak{B}}(\check{M})$ in $A d(M)$, and $V_{\mathfrak{B}}^{-}(A d(M))=$ $V_{\mathfrak{N}}(M) \otimes V_{\mathfrak{R}}(\check{M})$. If $A d(M)$ is critical at some integer, $F$ has to be totally real. We have the following result similarly to Theorem 5.2.1:

Theorem 5.2.2 - Suppose (Red $)_{+}$), ( \pm ) and (Reg) for rank 2 pure motive $M$. If $\operatorname{Ad}(M)(1)$ is critical, then $F$ is totally real, and we have

$$
c_{X, \sigma}^{+}(A d(M)(1))=c_{X, \sigma}^{+}(M) c_{X, \sigma}^{-}(M) \delta_{X, \sigma}(M(1))
$$




\subsection{Periods of $M_{1} \otimes \cdots \otimes M_{r}$}

We assume $\operatorname{rank}\left(M_{i}\right)=2$ and the following conditions

(Reg) $M_{i}$ is regular for all $i$,

(Cr) $M(1)$ is critical for $M=M_{1} \otimes \cdots \otimes M_{r}$;

$\left(\operatorname{Fr}_{M}\right) \mathscr{F}(M)$ is $E \otimes F$-free.

The assumption $\left(\operatorname{Fr}_{M}\right)$ is automatic under $( \pm)$ for $M_{i}$ if $F$ does not contain a CM field. When $F$ contains a CM field, we can always bring $M^{\prime}=M_{1}^{\prime} \otimes \cdots \otimes M_{r}^{\prime}$ satisfying (Reg) and ( $\mathrm{Cr}$ ) into $M$ satisfying the above three conditions by tensoring it with a rank $1 \mathrm{CM}$ motive; thus, periods of $M^{\prime}$ can be written down as a monomial of $c_{X, \sigma}^{+}\left(M_{i}\right), c_{X, \sigma}^{-}\left(M_{i}\right)$ and $\mathrm{CM}$ periods $\delta_{X, \sigma}\left(M_{i}\right)$. If $M$ has a non-trivial Hodge component $(q, q)$ at some place $\sigma \in I$, then for a Hodge type $\left(x_{i}, y_{i}\right)$ with $x_{i} \neq y_{i}$ of $M_{i}$ at $\sigma, \sum_{i} x_{i}=\sum_{i} y_{i}=q$ and $H^{q, q}\left({ }^{\sigma} M\right) \supset V_{1} \oplus V_{2}$ for $V_{1}=\otimes_{i} H^{x_{i}, y_{i}}\left({ }^{\sigma} M_{i}\right)$ and $V_{2}=\otimes_{i} H^{y_{i}, x_{i}}\left({ }^{\sigma} M_{i}\right)$. Since complex conjugation $c$ interchanges $V_{1}$ and $V_{2}, c$ does not act on $H^{q, q}(M)$ by scalar. Then the criticality of $M(1)$ prohibits this to happen, and thus $M$ satisfies $( \pm)$. We write $a_{i}(\sigma,+)=m_{i}(\sigma)$ and $a_{i}(\sigma,-)=n_{i}(\sigma)$. Following [5] and [74] Section 3, we consider the set $\Sigma=\{\{1, \ldots, r\} \rightarrow\{ \pm\}\}$ of functions and put

$$
\begin{aligned}
\Sigma_{\sigma}^{+}=\left\{s \in \Sigma \mid \sum_{i} a_{i}(\sigma, s(i))>\sum_{i} a_{i}(c \sigma,-s(i))\right\} & \\
& =\left\{s \in \Sigma \mid \sum_{j \in J(s)} k_{j}(\sigma)>\sum_{i \notin J(s)} k_{i}(\sigma)\right\},
\end{aligned}
$$

where $J(s)=\{j \mid s(j)=+\}$ and $k_{i}(\sigma)-1=a_{i}(\sigma,+)-a_{i}(c \sigma,-)$. From $m_{i}(\sigma)>n_{i}(\sigma)$, we have that $k_{i}(\sigma)>1$. Then $\mathscr{F}(M) \otimes_{E \otimes F, i_{\infty} \otimes \sigma} \mathbb{C}$ is equal to

$$
\sum_{s \in \Sigma_{\sigma}^{+}} \otimes_{i} H^{a_{i}(\sigma, s(i)), a_{i}(c \sigma,-s(i))}\left({ }^{\sigma} M_{i}\right)
$$

for all $\sigma \in I$. For $M$ to satisfy $\left(\operatorname{Red}_{+}\right), V_{\mathfrak{B}}(M)$ has to be a sum of tensor products of $H_{\mathfrak{p}}\left(M_{i}\right)$ and $V_{\mathfrak{B}}\left(M_{i}\right)$. This is equivalent to assuming

$\left(\operatorname{Red}_{ \pm}\right) \Sigma_{\sigma}^{+}$only depends on the p-adic place $\mathfrak{B}$ induced by $i_{p} \sigma$ for all $\sigma$.

Under $\left(\operatorname{Red}_{ \pm}\right)$, we write $\Sigma_{\mathfrak{B}}^{+}$for $\Sigma_{\sigma}^{+}$, and we have

$$
V_{\mathfrak{R}}(M)=V_{\mathfrak{P}}^{+}=V_{\mathfrak{B}}^{-}=\sum_{s \in \Sigma_{\mathfrak{B}}^{+}}\left(\otimes_{i \in J(s)} V_{\mathfrak{R}}\left(M_{i}\right)\right) \otimes\left(\otimes_{j \notin J(s)} H_{\mathfrak{p}}\left(M_{j}\right)\right) .
$$

We define for each $\sigma \in I$ the two integers $\nu_{i}(\sigma)$ and $\mu_{i}(\sigma)$ by

$$
\nu_{i}(\sigma)=\#\left\{s \in \Sigma_{\sigma}^{+} \mid s(i)=+\right\} \quad \text { and } \quad \mu_{i}(\sigma)=\#\left\{s \in \Sigma_{\sigma}^{+} \mid s(i)=-\right\} .
$$


Then $\mu_{i}(\sigma)+\nu_{i}(\sigma)=2^{r-1}$, and as shown in [5] and [74] Section 3, we have

Theorem 5.3.1 - Under the above assumption, we have $c_{X, \sigma}^{ \pm}\left(M_{1} \otimes M_{2} \otimes \cdots \otimes M_{r}\right)=\prod_{i}\left\{\left(c_{X, \sigma}^{+}\left(M_{i}\right) c_{X, \sigma}^{-}\left(M_{i}\right)\right)^{\left(\nu_{i}(\sigma)-\mu_{i}(\sigma)\right) / 2} \delta_{X, \sigma}\left(M_{i}\right)^{\mu_{i}(\sigma)}\right\}$

Although in [74] Section 3 p.156, it is assumed that $a_{i}(c \sigma, \pm)=a_{i}(\sigma, \pm)$ for all $\sigma$ to deduce Theorem 5.3.1, his proof works well in the general case as long as we assume $\left(\operatorname{Fr}_{M}\right)$.

\subsection{Conjectures on $\varphi_{1} \otimes \cdots \otimes \varphi_{r}$}

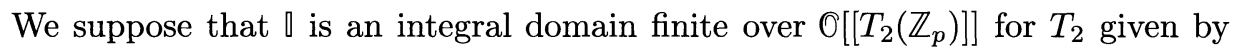
$\operatorname{Res}_{F / \mathbb{Q}} \mathbf{G}_{m} \times \mathbf{G}_{m}$. We consider arithmetic 0-adic representations $\varphi_{i}: \mathscr{G}_{F} \rightarrow G L_{2}(\mathbb{K})$ for $i=1,2, \ldots, r$. For each arithmetic point $P$, we write $(m(P), n(P))$ in $\mathbb{Z}[I]^{2}$ for its weight. Since $M_{i, P}$ is pure, $m_{\sigma}(P)+n_{c \sigma}(P)$ is independent of $\sigma$ and thus $m+c n$ factors through the torus $T$ defined in Section 2.1 of Chapter 2. We write $V_{\mathfrak{B}}\left(\varphi_{i}\right)$ for the space $V_{\mathfrak{B}}^{-}$specified in $\left(\mathrm{A} 2_{ \pm}\right)$for $\varphi_{i}$. Writing $\mathbb{L}$ for the field of fractions of $\llbracket \widehat{\otimes}_{0} \ldots \widehat{\otimes}_{\mathbb{0}} \rrbracket$, we have

$$
\varphi=\varphi_{1} \otimes \cdots \otimes \varphi_{r}: \mathscr{G}_{F} \rightarrow G L_{N}(\mathbb{L}) \quad \text { for } \quad N=2^{r}
$$

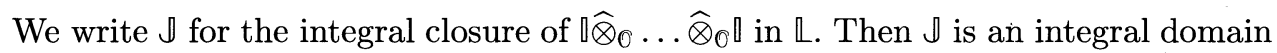
finite over $O\left[\left[T\left(\mathbb{Z}_{p}\right)\right]\right]$ for $T=T_{2}^{r} \cong T_{N}$, and $\varphi$ is arithmetic in the sense of Section 4.1 in Chapter 4. We have natural projection of $\mathscr{X}_{\sqrt{ }}$ to $\mathscr{X}_{\|}^{r}$. We have several different choices of $V_{\mathfrak{B}}=V_{\mathfrak{B}}^{ \pm}(\varphi)$. To describe this choice, we pick a subset $\Sigma_{\mathfrak{B}}^{+}$of $\Sigma$ for each $\mathfrak{B}$ such that

$$
\bigcap_{s \in \Sigma_{\mathfrak{B}}^{+}}\left\{x \in \mathbb{R}_{+}^{n} \mid \sum_{j} s(j) x_{j}>0\right\} \quad \text { is non-empty, and } \Sigma=\Sigma_{\mathfrak{B}}^{+} \bigsqcup-\Sigma_{\mathfrak{B}}^{+} .
$$

We write $S=\left\{\Sigma_{\mathfrak{B}}^{+} \mid \mathfrak{B} \in S_{F}\right\}$. For each $\mathfrak{B}$, there are $2^{r-1}$-choices of $\Sigma_{\mathfrak{B}}^{+}$. Thus the set of $\mathrm{S}$ is made of $2^{(r-1)\left|S_{F}\right|}$ elements. For each such choice $S$, we expect to have a $p$-adic $L$-function. These $p$-adic $L$-functions might be related in a yet unknown way. Let $V\left(\varphi_{j}\right)$ be the reflexive -module given for $\varphi_{j}$ in (Cont) in Section 4.1 of Chapter 4 and put $V(\varphi)$ for the $\mathbb{J}$-reflexive closure of the image of $\widehat{\otimes}_{j} V\left(\varphi_{j}\right)$ in $\left(\widehat{\otimes}_{j} V\left(\varphi_{j}\right)\right) \mathbb{Q} \mathbb{L}$. Then we define

$$
V_{\mathfrak{B}}(\varphi ; S)=V_{\mathfrak{B}}(\varphi)=V(\varphi) \bigcap\left\{\sum_{s \in \Sigma_{\mathfrak{B}}^{+}}\left(\widehat{\bigotimes}_{i \in J(s)} V_{\mathfrak{B}}^{-}\left(\varphi_{i}\right)\right) \widehat{\otimes}\left(\widehat{\bigotimes}_{j \notin J(s)} V\left(\varphi_{j}\right)\right) \otimes \mathbb{L}\right\} .
$$


We write an element $a=(m, n) \in X\left(T_{2}\right)$ as $m=\sum_{\sigma} a(\sigma,+) \sigma$ and $n=\sum_{\sigma} a(\sigma,-) \sigma$. Then under this convention, the subset of $X\left(T_{N}\right)=\oplus_{j} X\left(T_{2}\right)$ given by

$$
\Lambda_{S}=\left\{\left(a_{j}\right) \in \oplus_{j} X\left(T_{2}\right) \mid \sum_{i} a_{i}(\sigma, s(i))>\sum_{i} a_{i}(c \sigma,-s(i)) \quad \forall s \in \Sigma_{\mathfrak{R}}^{+} \quad \text { and } \quad \forall \sigma\right\}
$$

gives a spanning cone in $X(T) \otimes_{\mathbb{Z}} \mathbb{R}$ by (5.5.2). Here note that $\mathfrak{B}$ is induced by $i_{p} \sigma$. Thus the set of motivic point $P=\otimes_{i} P_{i} \in \mathscr{Q}_{0}^{r}$ such that $\sum_{i}\left(m\left(P_{i}\right), n\left(P_{i}\right)\right) \in \Lambda_{S}$ is dense in $\mathscr{X}_{0}^{r}$, and such points are dense in $\mathscr{L}_{\sqrt{\jmath}}$. We call such points motivic points of type $S$. This shows $\varphi$ satisfies $\left(\mathrm{A}^{\prime} 2_{ \pm}\right)$and (A3) for the above $\sqrt{ }$-submodule $V_{\mathfrak{B}}(\varphi ; S)$. The periods of $M_{P}=\otimes_{i} M_{i, P_{i}}$ for all motivic points $P$ of type $S$ have the same shape:

$$
c_{X, \sigma}^{ \pm}\left(M_{P}\right)=\prod_{i}\left\{\left(c_{X, \sigma}^{+}\left(M_{i, P_{i}}\right) c_{X, \sigma}^{-}\left(M_{i, P_{i}}\right)\right)^{\left(\nu_{i}(S, \sigma)-\mu_{i}(S, \sigma)\right) / 2} \delta_{X, \sigma}\left(M_{i, P_{i}}\right)^{\mu_{i}(S, \sigma)}\right\}
$$

where $\nu_{i}(S, \sigma)=\#\left\{s \in \Sigma_{\mathfrak{R}}^{+} \mid s(i)=+\right\}$ and $\mu_{i}(S, \sigma)=\#\left\{s \in \Sigma_{\mathfrak{R}}^{+} \mid s(i)=-\right\}$ for the place $\mathfrak{B}$ induced by $i_{p} \sigma$.

Conjecture 5.4.1 - For each choice $S$, we can find a unique p-adic L-function $L_{S, i_{\infty}}(\varphi)$ in the field of fractions of $\sqrt{\widehat{Q}_{\mathcal{O}} O_{\Omega}}$ such that for all motivic points $P$ of type $S$ critical at 1 , if the p-adic period does not vanish,

$$
i_{p}^{-1}\left(\frac{L_{S, i_{\infty}}(P, \varphi)}{c_{p, i_{\infty}}^{+}\left(\varphi_{P} \otimes \mathcal{N}^{-1}\right)}\right)=* i_{\infty}^{-1}\left(\frac{L_{i_{\infty}}\left(1, \varphi_{P}\right)}{c_{\infty, i_{\infty}}^{+}\left(\varphi_{P} \otimes \mathcal{N}^{-1}\right)}\right)
$$

where the period in the denominator is given by the formula $(P d)$.

This conjecture covers all critical values when $F$ does not contain a CM field. When $F$ contains a CM field, $\mathscr{F}\left(M_{P}\right)$ may not be free, and we have more cases to cover. To illustrate this, we describe the situation in a non-trivial case in the following paragraph.

\subsection{The case of $\varphi_{1} \otimes \varphi_{2}$}

First assume $\left(\operatorname{Fr}_{M}\right)$ in Section 5.3 for $r=2$. There are only 2 choices of $\Sigma_{\mathfrak{R}}^{+}$at each $\mathfrak{P}$. Writing $s \in \Sigma_{\mathfrak{R}}^{+}$as $(s(1) s(2))$, these two choices are $\{(++),(+-)\}$ and $\{(++),(-+)\}$. Let $S_{F}$ be the set of $p$-adic places of $F$. For an $S$, we define a subset $T$ of $S_{F}$ by $\mathfrak{P} \in T \Leftrightarrow(+-) \in \Sigma_{\mathfrak{R}}^{+}$and $\Sigma_{\mathfrak{R}}^{+} \in S$. For $\mathfrak{R} \in T$, we are in Case $T$ in Theorem 5.2.1 and for $\mathfrak{B} \notin T$, we are in Case $T$ for which the role of $M_{1}$ and $M_{2}$ is interchanged. The choice $S$ is determined by $T$. Writing $J(T)$ for the set of $\sigma \in I$ such that $i_{p} \sigma$ induces the $p$-adic places $\mathfrak{B}$ in $T$, we call a motivic point $P \otimes Q$ of 
type $T$ if

$$
\begin{gathered}
k_{\sigma}(P)>k_{c \sigma}(Q) \text { and } k_{c \sigma}(P)>k_{\sigma}(Q) \Longleftrightarrow \sigma \in J(T), \quad \text { and } \\
k_{\sigma}(Q)>k_{c \sigma}(P) \text { and } k_{c \sigma}(Q)>k_{\sigma}(P) \Longleftrightarrow \sigma \notin J(T),
\end{gathered}
$$

where $k_{\sigma}(P)-1=m_{\sigma}(P)-n_{c \sigma}(P)>1$. We now treat the general case without assuming $\left(\operatorname{Fr}_{M}\right)$. Thus we may assume that $F$ contains a $\mathrm{CM}$ field. We choose a partition $S_{F_{C M}}=A \sqcup c A \sqcup B \bigsqcup C \bigsqcup c C$, where $c$ is the generator of $\operatorname{Gal}\left(F_{C M} / F_{+}\right)$. We further choose a subset $T$ of $B$. Then the above partition induces a partition of $I$ by associating to $\sigma \in I$ a $p$-adic place of $F_{C M}$ induced by $\left.i_{p} \sigma\right|_{F_{C M}}$. We use the same symbols to write this partition of $I$; thus, $I=A \bigsqcup c A \bigsqcup T \bigsqcup(B-T) \bigsqcup C \bigsqcup c C$. We call a motivic point $P \otimes Q$ "of type $(A, T, C)$ " if

$$
\begin{aligned}
& \sigma \in A \Longleftrightarrow k_{c \sigma}(P)<-k_{c \sigma}(Q) \quad \text { and } \sigma \in c A \Longleftrightarrow k_{\sigma}(P)<-k_{\sigma}(Q) \\
& \sigma \in C \Longleftrightarrow k_{\sigma}(P)>k_{c \sigma}(Q) \quad \text { and } \quad k_{c \sigma}(P)<k_{\sigma}(Q) \\
& \sigma \in T \Longleftrightarrow k_{\sigma}(P)>k_{c \sigma}(Q) \text { and } k_{c \sigma}(P)>k_{\sigma}(Q) \\
& \sigma \in B-T \Longleftrightarrow k_{\sigma}(Q)>k_{c \sigma}(P) \quad \text { and } k_{c \sigma}(Q)>k_{\sigma}(P) .
\end{aligned}
$$

This implies that

$$
\begin{aligned}
& \mathfrak{P} \in T \Longleftrightarrow V_{\mathfrak{B}}\left(\varphi_{1} \otimes \varphi_{2}\right) \otimes \mathbb{L}=V_{\mathfrak{B}}\left(\varphi_{1}\right) \otimes V\left(\varphi_{2}\right) \otimes \mathbb{L} \\
& \mathfrak{B} \in B-T \Longleftrightarrow V_{\mathfrak{B}}\left(\varphi_{1} \otimes \varphi_{2}\right) \otimes \mathbb{L}=V\left(\varphi_{1}\right) \otimes V_{\mathfrak{B}}\left(\varphi_{2}\right) \otimes \mathbb{L} \\
& \mathfrak{B} \in A \Longleftrightarrow V_{\mathfrak{P}}\left(\varphi_{1} \otimes \varphi_{2}\right) \otimes \mathbb{L}=V\left(\varphi_{1}\right) \otimes V\left(\varphi_{2}\right) \otimes \mathbb{L} \\
& \mathfrak{B} \in c A \Longleftrightarrow V_{\mathfrak{B}}\left(\varphi_{1} \otimes \varphi_{2}\right) \otimes \mathbb{L}=\{0\} \\
& \mathfrak{P} \in C \Longleftrightarrow V_{\mathfrak{B}}\left(\varphi_{1} \otimes \varphi_{2}\right) \otimes \mathbb{L}=\left\{V\left(\varphi_{1}\right) \otimes V_{\mathfrak{B}}\left(\varphi_{2}\right)+V_{\mathfrak{\beta}}\left(\varphi_{1}\right) \otimes V\left(\varphi_{2}\right)\right\} \otimes \mathbb{L} \\
& \mathfrak{B} \in c C \Longleftrightarrow V_{\mathfrak{R}}\left(\varphi_{1} \otimes \varphi_{2}\right) \otimes \mathbb{L}=V_{\mathfrak{R}}\left(\varphi_{1}\right) \otimes V_{\mathfrak{R}}\left(\varphi_{2}\right) \otimes \mathbb{L} \text {. }
\end{aligned}
$$

When $F$ does not contain any CM field, we just pretend $A=C=\emptyset$, and we call a motivic point $P$ "of type $(\emptyset, T, \emptyset)$ " if it is of type $T$.

Conjecture 5.5.1 - For each choice of $S=(A, T, C)$, we find a p-adic L-function $L_{S, i_{\infty}}\left(\varphi_{1} \otimes \varphi_{2}\right)$ in the field of fractions of $\mathcal{\triangle}_{\otimes_{\mathcal{O}}} O_{\Omega}$ such that for all motivic points $P$ of type $S$ critical at 1 , if the p-adic period does not vanish,

$$
i_{p}^{-1}\left(\frac{L_{S, i_{\infty}}\left(P \otimes Q, \varphi_{1} \otimes \varphi_{2}\right)}{c_{p, i_{\infty}}^{+}\left(\varphi_{1, P} \otimes \varphi_{2, Q} \otimes \mathcal{N}^{-1}\right)}\right)=* i_{\infty}^{-1}\left(\frac{L_{i_{\infty}}\left(1, \varphi_{1, P} \otimes \varphi_{2, Q}\right)}{c_{\infty, i_{\infty}}^{+}\left(\varphi_{1, P} \otimes \varphi_{2, Q} \otimes \mathcal{N}^{-1}\right)}\right)
$$


where we have

$$
\begin{aligned}
c_{X, i_{\infty}}^{+}\left(\varphi_{1, P} \otimes \varphi_{2, Q} \otimes \mathcal{N}^{-1}\right) & =\prod_{\sigma \in A} \delta_{X, \sigma}\left(\varphi_{1, P}\right)^{2} \delta_{X, \sigma}\left(\varphi_{2, Q} \otimes \mathcal{N}^{-1}\right)^{2} \\
& \times \prod_{\sigma \in T /\langle c\rangle} c_{X, \sigma}^{+}\left(\varphi_{1, P}\right) c_{X, \sigma}^{-}\left(\varphi_{1, P}\right) \delta_{X, \sigma}\left(\varphi_{2, Q} \otimes \mathcal{N}^{-1}\right) \\
& \times \prod_{\sigma \in B-T /\langle c\rangle} c_{X, \sigma}^{+}\left(\varphi_{2, Q}\right) c_{X, \sigma}^{-}\left(\varphi_{2, Q}\right) \delta_{X, \sigma}\left(\varphi_{1, P} \otimes \mathcal{N}^{-1}\right) \\
& \times \prod_{\sigma \in C} c_{X, \sigma}^{+}\left(\varphi_{1, P}\right) c_{X, \sigma}^{-}\left(\varphi_{2, Q}\right) \delta_{X, c \sigma}\left(\varphi_{1}, Q\right) \delta_{X, c \sigma}\left(\varphi_{2, Q} \otimes \mathcal{N}^{-1}\right) .
\end{aligned}
$$

\subsection{The case of $\operatorname{Ad}(\varphi)$}

We write $\varphi=\varphi_{1}$ and consider the tensor product $\varphi \otimes_{\mathbb{1}} \check{\varphi}$. In $V\left(\varphi \otimes_{\mathbb{0}} \check{\varphi}\right)=$ $\operatorname{End}_{\mathbb{1}}(V(\varphi))$, there is a unique 3 dimensional subspace $V(A d(\varphi))$ with trace 0 endomorphisms, giving the following decomposition over $\mathbb{K}: \varphi \otimes_{\mathbb{l}} \check{\varphi} \cong \operatorname{Ad}(\varphi) \oplus \mathbf{1}$. As seen in Theorem 5.2.2, for $A d(\varphi)$ being arithmetic, we need to assume that $F$ is totally real. In this case, there is only one choice of $S=\left\{V_{\mathfrak{R}}^{+}(\operatorname{Ad}(\varphi)), V_{\mathfrak{R}}^{-}(\operatorname{Ad}(\varphi))\right\}_{\mathfrak{B}}$ for a given choice of $\left\{V_{\Re}(\varphi)\right\}_{\Re}$. Thus we expects only one genuine $p$-adic $L$-function $L_{p, i_{\infty}}(A d(\varphi))$ for each $i_{\infty}$. Thus for $S=(\emptyset, I, \emptyset)$, by Theorems 5.2.1 and 5.2.2, the ratio $\frac{L_{S, i_{\infty}}\left(\varphi_{1} \otimes \varphi_{2}\right)}{L_{p, i_{\infty}}\left(\operatorname{Ad}\left(\varphi_{1}\right)\right)}$, which is equal to $\frac{L_{S}\left(\varphi_{1} \otimes \varphi_{2}\right)}{L_{p}\left(\operatorname{Ad}\left(\varphi_{1}\right)\right)}$ by (NP) in Section 4.3 of Chapter 4

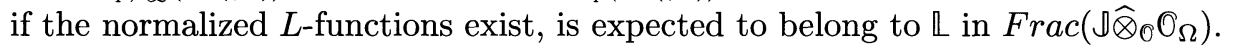

We now assume that $p>2$ and $\left(\mathrm{Fr}_{\mathbb{1}}\right),\left(\mathrm{FR}_{ \pm}\right)$for $\varphi$ in Section 4.3. Then $\operatorname{Ad}(\varphi)$ automatically satisfies these conditions. We further suppose the absolute irreducibility (AI) for $\bar{\varphi}$. By [54] Lemma 2 in Section 1.2, $A d(\varphi)_{P}$ for any $P \in$ $\mathscr{Q}_{\mathbb{1}}$ never has the trivial subquotient. If $\operatorname{Ad}(\varphi)_{P}^{*}=\operatorname{Ad}(\varphi)_{P} \otimes \mathcal{N}$ contains the trivial subquotient, then $\bar{\varphi} \cong \bar{\varphi} \otimes \mathcal{N}$, which implies that $p=3$ and $\bar{\varphi}$ is an induced representation of a character of $\mathscr{G}_{F(\sqrt{-3})}$. Thus if $p \geq 5$ or $\bar{\varphi}$ is not an induced representation of a character of $\varphi_{F(\sqrt{-3})}, A d(\varphi)_{P}^{*}$ never contains the trivial representation. Here is a refinement of Conjecture 1.0.1:

Conjecture 5.6.1 - Suppose (AI) for $\varphi \in \pi(\lambda)$ for a primitive algebra homomor-

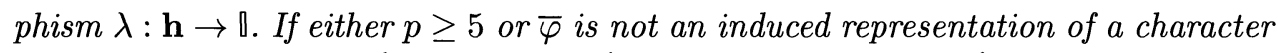
of $\mathscr{G}_{F(\sqrt{-3})}$, then over $\square \widehat{\otimes}_{\mathcal{O}} \widehat{O}_{\Omega}, C_{0}(\lambda ; \square) \widehat{\otimes}_{\odot} \bigcirc_{\Omega}$ is isomorphic to $\square \widehat{\otimes}_{\mathcal{O}} \widehat{O}_{\Omega} /\left(L_{p}(A d(\varphi))\right)$, where $L_{p}(\operatorname{Ad}(\varphi))$ is the normalized genuine $p$-adic L-function of $\operatorname{Ad}(\varphi)$.

\subsection{The case of $\varphi_{1} \otimes \varphi_{2} \otimes \varphi_{3}$}

We now specialize Conjecture 5.4.1 to $F=\mathbb{Q}$ and $r=3$. We write $k_{i}$ for $k_{i}(\sigma)$ with the unique embedding $\sigma$ of $\mathbb{Q}$ into $\overline{\mathbb{Q}}$. We write $Q_{X}\left(M_{i}\right)$ for $c_{X, \sigma}^{+}\left(M_{i}\right) c_{X, \sigma}^{-}\left(M_{i}\right)$. There are 4 different types $S$, and three out of the four types are transformed each 
other by permuting $M_{1}, M_{2}$ and $M_{3}$ as follows [5]:

\begin{tabular}{|c|c|}
\hline Type & Period \\
\hline (i) $k_{1}>k_{2}+k_{3}$ & $Q_{X}\left(M_{1}\right)^{2} \delta_{X, \sigma}\left(M_{2}\right)^{2} \delta_{X, \sigma}\left(M_{3}\right)^{2}$ \\
\hline $\begin{array}{c}\text { (ii) } k_{1}+k_{2}>k_{3}, k_{1}+k_{3}>k_{2}, \\
\text { and } k_{2}+k_{3}>k_{1}\end{array}$ & $\prod_{j=1}^{3} Q_{X}\left(M_{j}\right) \delta_{X, \sigma}\left(M_{j}\right)$ \\
\hline
\end{tabular}

Thus there should be 3 -variable $p$-adic $L$-functions of 4 different types. When $\varphi_{i} \in \pi\left(\lambda_{i}\right)$ for algebra homomorphisms $\lambda_{i}: h^{n . o r d}\left(N_{i} p^{\infty} ; \mathcal{O}\right) \rightarrow \mathbb{1}, p$-adic $L$-functions related to $\varphi_{1} \otimes \varphi_{2} \otimes \varphi_{3}$ has been constructed in [32] and [57]. Assuming $N_{i}=1$, Panchishkin [57] deals with the case (ii). His $p$-adic $L$-function is a function sending characters $\chi$ of $\mathbb{Z}_{p}^{\times}$to the $L$-value $L\left(1, \varphi_{P \otimes Q \otimes R} \otimes \chi\right)$ divided by a suitable period for a fixed a motivic point $P \otimes Q \otimes R$ of type (ii). Thus our conjecture asserts that Panchishkin's $L$-functions can be glued together to yield a 3 -variable type (ii) $L$-function. I should also mention that the result in [57] is valid without assuming $\left(\operatorname{Red}_{ \pm}\right)$. On the other hand, Harris and Tilouine treat the case (i) interpolating along $\operatorname{Spec}\left(h^{n . o r d}\left(N_{1} p^{\infty} ; \mathcal{O}\right)\right)$ square roots of type (i) values. They get a $p$-adic $L$ function which sends $P$ to $\sqrt{L\left(\frac{m(P)+n(P)+1}{2}, \varphi_{P \otimes Q \otimes R}\right)}$ divided basically by the Petersson self inner product of the modular form corresponding to $P$ for a fixed $Q \otimes R$. Their evaluation point is the center of symmetry of the functional equation of $L\left(s, \varphi_{P \otimes Q \otimes R}\right)$. Thus our conjecture asserts the extensibility of the square of the $L$ function to a 3 variable $L$-function $L^{(i)}\left(\varphi_{1} \otimes \varphi_{2} \otimes \varphi_{3}\right)$. Similarly to Conjecture 5.6.1, we expect that the ratio $\frac{L^{(i)}\left(\varphi_{1} \otimes \varphi_{2} \otimes \varphi_{3}\right)}{L_{p}\left(\operatorname{Ad}\left(\varphi_{1}\right)\right)^{2}}$ falls in $\mathbb{L}$. 



\section{6. $p$-Adic Rankin products}

We assume that $F$ is totally real. We study the $p$-adic $L$-functions constructed in [41] in terms of the genuine $p$-adic $L$-functions of $\varphi \otimes \check{\rho}$ for two modular Galois representations.

\subsection{Complex Rankin products}

We write $\mathbf{h}(N)$ (resp. $\boldsymbol{\Lambda})$ for $h^{\text {n.ord }}\left(N p^{\infty} ; \mathcal{O}\right)$ or $h^{\text {ord }}\left(N p^{\infty} ; \mathcal{O}\right)\left(\right.$ resp. $\Lambda$ or $\Lambda^{\text {ord }}$ ) as in Section 2.9 of Chapter 2. Let $\lambda: \mathbf{h}(N) \rightarrow \llbracket$ and $\mu: \mathbf{h}(J) \rightarrow \llbracket$ be primitive $\boldsymbol{\Lambda}$-algebra homomorphisms giving irreducible components of the Hecke algebras. Take $\varphi$ (resp. $\rho$ ) in the isogeny class $\pi(\lambda)$ (resp. $\pi(\mu)$ ). For two positive arithmetic points $P$ and $Q$ of $\mathscr{L}_{\mathbb{1}}$, we look at the complex $L$-function $L\left(s, \varphi_{P} \otimes \check{\rho}_{Q}\right)$. This $L$ function is convergent if $\operatorname{Re}(s)$ is sufficiently large, is continued to the whole $s$-plane as a meromorphic function and satisfies the functional equation as in Section 3.1 of Chapter 3 (see [51]).

The principle $\left(\mathrm{AT}_{\infty}\right)$ in Section 4.4 of Chapter 4 is known to hold for $L\left(s, \varphi_{P} \otimes\right.$ $\left.\check{\rho}_{Q}\right)$. This is equivalent to saying that $L\left(s, \varphi_{P} \otimes \check{\rho}_{Q}\right)$ has a pole at $s=1$ if and only if $\varphi_{P} \cong \rho_{Q}$ because the two representations are absolutely irreducible (see [65] Proposition 4.13). When $\varphi_{P} \otimes \check{\rho}_{Q} \otimes \mathcal{N}^{-1}$ is critical, as conjectured by Shimura and proven by Shimura, Harris and Yoshida ([65], [66], [67], [30], [31] and [74], [75]), the transcendental factor of $L(1, \varphi \otimes \check{\rho})$ has a natural factorization described in Section 5.2 of Chapter 5 depending on the two weights $\kappa(P)$ and $\kappa(Q)$ of $P$ and $Q$, respectively. We look into the following special case of type $(\emptyset, I, \emptyset)$ :

$$
m(P)-m(Q)>0 \geq n(P)-n(Q),
$$

where the transcendental factor $c_{X}^{+}\left(\varphi_{P} \otimes \check{\rho}_{Q} \otimes \mathcal{N}^{-1}\right)$ is very close to $c_{X}^{+}\left(\operatorname{Ad}\left(\varphi_{P}\right) \otimes \mathcal{N}^{-1}\right)$ as shown in Theorems 5.2.1 and 5.2.2 of Chapter 5. The algebraicity theorem in this case is studied in [65] (see also [41]), and the transcendental factor is given, up to a power of $\pi$, by the self Petersson inner product $\left(f_{P}, f_{P}\right)$ of a Hilbert cusp form $f_{P}$, where $f_{P}$ is the primitive element in the automorphic representation generated 
by the value $f(P)$ at $P$ of the -adic form $f$ giving rise to $\varphi$. On the other hand, as shown in [47] Theorem 7.1,

$$
L_{i_{\infty}}\left(1, A d\left(\varphi_{P}\right)\right)=* \pi^{m(P)-n(P)+2 t}\left(f_{P}, f_{P}\right),
$$

where $\pi^{m}=\prod_{\sigma \in I} \pi^{m_{\sigma}}$ and $*$ is a simple constant depending only on $\kappa(P)$ and the conductor of $f_{P}$. This combined with Theorem 4.2 of [65] (see also p.320 of [41]) shows that under the assumption of $(\mathrm{Ad})$

$$
\frac{G\left(\operatorname{det}\left(\rho_{Q}\right)\right) L_{i_{\infty}}\left(1, \varphi_{P} \otimes \check{\rho}_{Q}\right)}{(2 \pi i)^{\{\kappa(Q)\}-\{\kappa(P)\}} G\left(\operatorname{det}\left(\varphi_{P}\right)\right) L_{i_{\infty}}\left(1, A d\left(\varphi_{P}\right)\right)} \in \overline{\mathbb{Q}},
$$

where $G\left(\operatorname{det}\left(\rho_{Q}\right)\right)$ is the Gauss sum of the complex avatar of the character $\operatorname{det}\left(\rho_{Q}\right)$ specified in [65] (3.9). By definition, $\delta_{*}\left(M\left(\check{\rho}_{Q} \otimes \mathcal{N}^{-1}\right)\right)=G\left(\operatorname{det}\left(\rho_{Q}\right)\right)^{-1} x^{2 t+\{\kappa(Q)\}}$ for $x=2 \pi i$ or 1 according as $*=\infty$ or $p$. This follows from the fact that

(i) the Tate motive $T=\mathbb{Q}(1)$ is given by $H_{p}(T)=\mathbb{Q}_{p}(1), H_{B}(T)=(2 \pi i) \mathbb{Q} \subset \mathbb{C}$ and $H_{D R}(T)=\mathbb{Q}$, and

(ii) we have identified $\mathbb{Q}_{p}(1)$ with $\mathbb{Q}_{p}$.

The fact (R1) is well explained by the following result deduced from Theorems 5.2.1 and 5.2.2 in Chapter 5:

Proposition 6.1.1 - Suppose (Ad) for $P \otimes Q$. Then we have

$$
\begin{gathered}
(2 \pi i)^{-\{\kappa(Q)\}} G\left(\operatorname{det}\left(\rho_{Q}\right)\right) c_{\infty}^{+}\left(\varphi_{P} \otimes \check{\rho}_{Q} \otimes \mathcal{N}^{-1}\right) \\
=(2 \pi i)^{-\{\kappa(P)\}} G\left(\operatorname{det}\left(\varphi_{P}\right)\right) c_{\infty}^{+}\left(\operatorname{Ad}\left(\varphi_{P}\right) \otimes \mathcal{N}^{-1}\right) \\
G\left(\operatorname{det}\left(\rho_{Q}\right)\right) c_{p}^{+}\left(\varphi_{P} \otimes \check{\rho}_{Q} \otimes \mathcal{N}^{-1}\right)=G\left(\operatorname{det}\left(\varphi_{P}\right)\right) c_{p}^{+}\left(A d\left(\varphi_{P}\right) \otimes \mathcal{N}^{-1}\right) .
\end{gathered}
$$

This shows that $\frac{L_{S, i_{\infty}}(\varphi \otimes \check{\rho})}{L_{p, i_{\infty}}(\operatorname{Ad}(\varphi))}$ actually falls in the field of fractions of $\square \widehat{\otimes}_{\mathbb{O}} \llbracket$. If there exist the normalized genuine $p$-adic $L$-functions $L_{S}(\varphi \otimes \check{\rho})$ of type $(\emptyset, I, \emptyset)$ and $L_{p}(A d(\varphi))$, by $(\mathrm{NP})$ in Section 4.3 of Chapter $4, \frac{L_{S, i_{\infty}}(\varphi \otimes \check{\rho})}{L_{p, i_{\infty}}(\operatorname{Ad}(\varphi))}$ is equal to $\frac{L_{S}(\varphi \otimes \check{\rho})}{L_{p}(\operatorname{Ad}(\varphi))}$ and is independent of $i_{\infty}$ and the choice of $\varphi \in \pi(\lambda)$ and $\rho \in \pi(\mu)$.

\subsection{The $p$-adic Rankin product in [41]}

When $\mathbf{h}=h^{\text {n.ord }}$, we constructed in [41] a $p$-adic (meromorphic) $L$-function $D_{p}(\pi(\lambda) \otimes \check{\pi}(\mu)): \mathscr{X}_{\mathbb{}} \times \mathscr{X}_{\mathbb{1}} \rightarrow \Omega$ in $\mathbb{L}$ satisfying the following interpolation property: up to a $p$-Euler factor and a simple algebraic constant $*$

$$
D_{p}(P, Q ; \pi(\lambda) \otimes \check{\pi}(\mu))=* i_{\infty}^{-1}\left(\frac{G\left(\operatorname{det}\left(\pi\left(\rho_{Q}\right)\right)\right) L_{i_{\infty}}\left(1, \varphi_{P} \otimes \check{\rho}_{Q}\right)}{G\left(\operatorname{det}\left(\pi\left(\varphi_{P}\right)\right)\right)(2 \pi i)^{\{\kappa(Q)\}-2 n(P)+2 t}\left(f_{P}, f_{P}\right)}\right)
$$


for motivic $(P, Q) \in \mathscr{A}_{\mathbb{0}} \times \mathscr{A}_{\mathbb{1}}$ satisfying (Ad) for $\kappa=\kappa(P)$ and $\kappa^{\prime}=\kappa(Q)$. The constant $*$ can be checked to be the value $u(P \otimes Q)$ of a unit $u \in(\boldsymbol{\Lambda} \widehat{\otimes} \boldsymbol{\Lambda})^{\times}$ independent of $P \otimes Q$ after factoring out the canonical $p$-Euler factors which should appear in two genuine $p$-adic $L$-functions (see Conjecture 4.2.1 in Chapter 4 ), although the computation is a bit involved. For this fact, see [47] (8.14a). Although in [47], we have only dealt with the CM case, the computation in general is essentially the same (except possibly at places dividing 2) because at each (odd finite) place, the local automorphic representation is of CM type (that is, it is induced from a character of a quadratic extension). This combined with (AV) simply shows that $D_{p}(\pi(\lambda) \otimes \check{\pi}(\mu))$ gives, up to unit factors, the ratio $\frac{L_{S, i_{\infty}}(\varphi \otimes \check{\rho})}{L_{p, i_{\infty}}(\operatorname{Ad}(\varphi))}$ for $S=(\emptyset, I, \emptyset)$ if two genuine $p$-adic $L$-functions exist.

To support this idea, we look into the singularity of $D_{p}(\pi(\lambda) \otimes \check{\pi}(\mu))$. As we will see in the following paragraph, admitting $\left(\operatorname{AT}_{p}\right), L_{S}(\varphi \otimes \check{\rho})$ is supposed to be everywhere holomorphic in the nearly ordinary case, and in the ordinary case, $L_{S}(\varphi \otimes \check{\rho})$ can have singularity only at the diagonal divisor $\Delta$ of $\mathscr{X}_{\mathbb{1}} \times \mathscr{X}_{\mathbb{1}}$ when $\varphi=\rho$. The singularity of $D_{p}(\pi(\lambda) \otimes \check{\pi}(\mu))$ is not as above. Via the theory of congruence modules (cf. see Section 2.9 of Chapter 2), the locus of points $P$ for which there exists $\nu: h^{n . o r d} \rightarrow \square$ such that $\nu_{P}=\lambda_{P}$ is the support $X$ of the congruence module $C(\lambda)=C_{0}(\lambda ; \square)$ of $\lambda$. In particular, we know from its construction in [41] that the singular locus of $D_{p}(\pi(\lambda) \otimes \check{\pi}(\mu))$ is contained in $X \times \mathscr{X}_{\mathbb{1}}$. In the CM case, we have an exact information of $X$, and $X \times \mathscr{Q}_{\mathbb{D}}$ is really different from $\Delta$. As explained in Section 2.10 of Chapter 2 and Conjecture 5.6.1 in Chapter 5, the characteristic ideal

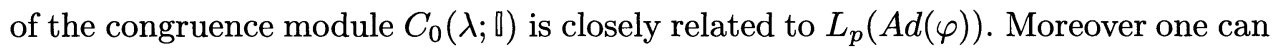
check in this modular case (see Theorem I and Lemma 5.3 of [41]), the removed $p$-Euler factor is identical to the ratio of those Euler $p$-factors of $L_{S}(\varphi \otimes \check{\rho})$ and $L_{p}(\operatorname{Ad}(\varphi))$ as predicted by Coates and Perrin-Riou (see (iii) of Conjecture 4.2.1) for cyclotomic twists $P \otimes Q$ of crystalline motivic points. Thus we expect that $D_{p}$ is the ratio $\frac{L_{S}(\varphi \otimes \check{\rho})}{L_{p}(A d(\varphi))}$ of two genuine $p$-adic $L$-functions. Here the numerator $L_{S}(\varphi \otimes \check{\rho})$ should be legitimately called the $p$-adic Rankin product of type $(\emptyset, I, \emptyset)$.

\subsection{Ordinary versus nearly ordinary}

Take a prime divisor $\mathbf{P}$ of $\mathscr{X}_{\mathbb{1}} \times \mathscr{X}_{\mathbb{1}}$, and write $k(\mathbf{P})$ for its residue field. It is easy to see that $V((\varphi \otimes \check{\rho}) \mathbf{P}) \otimes k(\mathbf{P})$ and $V\left((\varphi \otimes \check{\rho})_{\mathbf{P}}^{*} \otimes k(\mathbf{P})\right.$ never have the trivial subquotient for prime divisors $\mathbf{P}$ of the form $P \times \mathscr{Q}_{\mathbb{1}}$ or $\mathscr{Q}_{\mathbb{1}} \times Q$. Thus if they have the trivial subquotient, the projection of $\mathbf{P}$ to each component is dominant, and we find densely populated prime divisors $P$ and $Q$ on $\mathscr{L}_{\mathbb{Q}}$ such that $P \times Q$ lies on $\mathbf{P}$ and one of $\varphi_{P}$ and $\rho_{Q}$ is irreducible. Then $V\left(\varphi_{P} \otimes \check{\rho}_{Q}\right) \cong \operatorname{Hom}\left(V\left(\rho_{Q}\right), V\left(\varphi_{P}\right)\right)$ has the trivial subquotient if and only if $\varphi_{P} \cong \rho_{Q}$. Thus, in this case, $\mathbf{P}$ has to be the 
diagonal divisor $\Delta$ and $\operatorname{dim}_{\mathscr{O}}\left(\mathscr{Q}_{\mathbb{D}}\right)=1$. By a similar argument, if $V\left((\varphi \otimes \check{\rho})^{*}\right) \mathbf{P} \otimes k(\mathbf{P})$ has the trivial subquotient for a prime divisor $\mathbf{P}, \mathbf{P}(\Omega)=\left\{\left(P, P \otimes \mathcal{N}^{-1}\right) \mid P \in \mathscr{X}\right\}$ and $\operatorname{dim}_{\mathscr{C}} \mathscr{Q}_{\mathbb{1}}=1$. As in Section 2.5 of Chapter 2, writing $d$ for the defect of the Leopoldt conjecture for $(p, F)$, we know that $\operatorname{dim}_{\mathscr{O}}\left(h^{n . o r d}\right)=[F: \mathbb{Q}]+1+d$ and $\operatorname{dim}_{\mathscr{O}}\left(h^{\text {ord }}\right)=1+d$. By (Gal) (iv) in Section 2.8 of Chapter 2 , the inertia group $I_{\mathfrak{B}}$ at $\mathfrak{B} \mid p$ acts on a one dimensional subspace of $V_{\mathfrak{B}}^{-}(\lambda)=V_{\mathfrak{\beta}}^{-}(\varphi)$ by a character $\delta$ such that $\delta(y)=\lambda\left(\mathbb{T}(y)\right.$ ), where we identified $I_{\mathfrak{B}}$ with $\mathfrak{r}_{\mathfrak{B}}^{\times}$(resp. $I_{p}=\prod_{\mathfrak{B} \mid p} I_{\mathfrak{R}}$ with $\mathrm{r}_{p}^{\times}$) by class field theory. This shows us that $\delta$ coincides with the character: $I_{p} \rightarrow \mathbb{O}[[\mathbf{G}]]^{\times}$given by $y \mapsto(y, 1) \in \mathbf{G}$. On the other hand, on $I_{p}$, we have $\operatorname{det}(\varphi)(z)=\langle z\rangle \in \mathbb{O}[[\mathbf{G}]]^{\times}$. Therefore $\varphi_{P}=\rho_{Q}$ implies $\kappa(P)=\kappa(Q)$. Similarly, $\varphi_{P}=\rho_{Q} \otimes \mathcal{N}$ implies $\kappa(P)=\kappa(Q) \otimes(t, t)$. The locus of $(P, Q)$ with $\kappa(P)=\kappa(Q) \otimes(t, t)$ is of codimension $>1$. The diagonal locus of $\kappa(P)=\kappa(Q)$ is a divisor of $\mathscr{X}_{\mathbb{1}} \times \mathscr{X}_{\mathbb{D}}$ if and only if we are in the ordinary case and $\delta$ is trivial on $I_{p}$. Thus we may make the following conjectures for $\mathbb{1}$ finite and torsion-free over $\Lambda^{\text {ord }}$ :

Conjecture 6.3.1 - (Ordinary case) Take primitive $\Lambda^{\text {ord }}$-algebra homomorphisms $\lambda: h^{\text {ord }}\left(N p^{\infty} ; \mathcal{O}\right) \rightarrow \llbracket$ and $\mu: h^{\text {ord }}\left(J p^{\infty} ; \mathbb{O}\right) \rightarrow \llbracket$. Let $S=(\emptyset, I, \emptyset)$. Suppose that the existence of genuine $p$-adic L-functions $L_{S, i_{\infty}}(\varphi \otimes \check{\rho})$ of type $S$ and $L_{p, i_{\infty}}(\operatorname{Ad}(\varphi))$ for the Galois representations $\varphi \in \pi(\lambda)$ and $\rho \in \pi(\mu)$. Then

$$
L_{S, i_{\infty}}(\varphi \otimes \check{\rho}) \text { is finite outside the diagonal } \Delta \text { in } \mathscr{X}_{\mathbb{1}} \times \mathscr{X}_{\mathbb{1}} \text {; }
$$

(ii) $L_{S, i_{\infty}}(\varphi \otimes \check{\rho})$ has singularity at $\Delta$ in $\mathscr{L}_{\mathbb{l}} \times \mathscr{L}_{\mathbb{l}}$, which is actually a simple pole, if and only if $\lambda=\mu$ and the Leopoldt conjecture holds for $p$ and $F$;

(iii) $D_{p}^{\text {ord }}(\pi(\lambda) \otimes \check{\pi}(\mu))=\frac{L_{S, i_{\infty}(\varphi \otimes \check{\rho})}}{L_{p, i_{\infty}}(\operatorname{Ad}(\varphi))}$ up to finite Euler factors outside $p$ and [-units,

where $D_{p}^{\text {ord }}(\pi(\lambda) \otimes \check{\pi}(\mu))$ is the ordinary version of $D_{p}(\pi(\lambda) \otimes \check{\pi}(\mu))$ which is constructed in [37] when $F=\mathbb{Q}$ and will be constructed in the following sections for general $F$.

The complex $L$-values as in (Int1) may not always be the one of primitive $L$ functions. Because of this defect, we cannot really claim that the identity in (iii) is only up to units. By (NP) in Chapter 4 and Theorems 5.2.1 and 5.2.2 of Chapter 5, $\alpha_{ \pm}(\varphi \otimes \check{\rho})=\alpha_{+}(\varphi) \alpha_{-}(\varphi)=\alpha_{+}(\operatorname{Ad}(\varphi))$ for $S=(\emptyset, I, \emptyset)$, and hence we have

$\operatorname{ord}_{\Delta} L_{S, i_{\infty}}(\varphi \otimes \check{\rho})=\operatorname{ord}_{\Delta} L_{S}(\varphi \otimes \check{\rho}) \quad$ and $\quad \operatorname{ord}_{\Delta} L_{p, i_{\infty}}(\operatorname{Ad}(\varphi))=\operatorname{ord}_{\Delta} L_{p}(\operatorname{Ad}(\varphi))$.

We have proven in [37] when $F=\mathbb{Q}$ that the ordinary version $D_{p}^{\text {ord }}(\pi(\lambda) \otimes \check{\pi}(\mu))$ has a simple pole at the diagonal if and only if $\lambda=\mu$. The technique of [37] can be generalized for general totally real $F$ as we shall give a proof later in this chapter (see Sections 6.4-6.10). Putting the proof off to these sections, here we state only the outcome. For that, we prepare some notation. Since we are in the ordinary case, we have well defined Hecke operator $\mathbb{T}(\mathfrak{B}) \in h^{\text {ord }}$ for each prime factor $\mathfrak{B}$ of $p$ and 
$\lambda(\mathbb{T}(\mathfrak{B}))$ and $\mu(\mathbb{T}(\mathfrak{P}))$ are units in $\mathbb{0}$. Since $\operatorname{Spec}\left(h^{\text {ord }}(N)\right)$ is a closed subscheme of

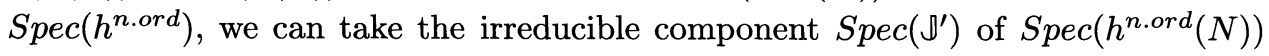
through which $\lambda$ factors. Similarly we take the irreducible component $\operatorname{Spec}\left(\mathbb{J}^{\prime \prime}\right)$ of $\operatorname{Spec}\left(h^{\text {n.ord }}(J)\right)$ through which $\mu$ factors. Thus we can take a common normal finite extension $\mathbb{J}$ of $\mathbb{J}^{\prime}$ and $\mathbb{J}^{\prime \prime}$ so that $\operatorname{Spec}(\mathbb{\square})$ is a closed subscheme of $\operatorname{Spec}(\mathbb{J})$. Then we take the $\Lambda$-algebra homomorphisms $\boldsymbol{\lambda}: h^{\text {n.ord }}(N) \rightarrow \mathbb{J}$ and $\boldsymbol{\mu}: h^{\text {n.ord }}(J) \rightarrow \mathbb{J}$ which induces $\lambda$ and $\mu$ on the ordinary quotient. By [41] Theorem I, we have the nearly ordinary $p$-adic Rankin product $D_{p}(\pi(\boldsymbol{\lambda}) \otimes \check{\pi}(\boldsymbol{\mu}))$ in the quotient field of $\mathbb{\mathbb { } \otimes} \mathbb{J}$. Then we can think of the restriction of $D_{p}(\pi(\boldsymbol{\lambda}) \otimes \check{\pi}(\boldsymbol{\mu}))$ to $\mathscr{Q}_{\mathbb{0}} \times \mathscr{X}_{\mathbb{0}}$, for which we write $D_{p}^{\text {ord }}(\pi(\boldsymbol{\lambda}) \otimes \check{\pi}(\boldsymbol{\mu}))$. We know that $\Lambda^{\text {ord }}=\mathcal{O}\left[\left[\mathbf{W}^{\text {ord }}\right]\right]$. If $\mathbf{W}^{\text {ord }} \cong \mathbb{Z}_{p}(\Leftrightarrow d=0)$, we pick a generator $w$ of $\mathbf{W}^{\text {ord }}$; otherwise, we just pick one of generators $w$ of $\mathbf{W}^{\text {ord }}$ such that $\mathcal{N}(w)$ generates $\mathcal{N}\left(\mathbf{W}^{\text {ord }}\right)$. Then, if $d=0, t_{w}=w^{-1} \otimes w-1$ in $\Lambda^{\text {ord }} \widehat{\otimes} \Lambda^{\text {ord }}$ is a local parameter of $\Delta$. For every function $f$ on $\mathscr{X}_{\mathbb{1}} \times \mathscr{X}_{\mathbb{1}}$ with at worst a simple pole at $\Delta, \operatorname{Res}_{\Delta} f=\left.\left(t_{w} f / \log _{p} \mathcal{N}(w)\right)\right|_{t_{w}=0}$ is a function on $\Delta$ independent of the choice of $w$.

Theorem 6.3.2 — Let $\lambda: h^{\text {ord }}\left(N p^{\infty} ; \mathcal{O}\right) \rightarrow \llbracket$ and $\mu: h^{\text {ord }}\left(J p^{\infty} ; \mathcal{O}\right) \rightarrow \square$ be primitive $\Lambda^{\text {ord }}$-algebra homomorphisms. Then there exists $D_{p}^{\text {ord }}(\pi(\lambda) \otimes \check{\pi}(\mu))$ in the field of fractions of $\square \widehat{\otimes} \mathbb{Q}$ satisfying the following properties:

$$
D_{p}^{\text {ord }}(\pi(\lambda) \otimes \check{\pi}(\mu))=\prod_{\mathfrak{B} \mid p}(1-\lambda(\mathbb{T}(\mathfrak{P})) / \mu(\mathbb{T}(\mathfrak{P})))^{-1} D_{p}^{\text {ord }}(\pi(\boldsymbol{\lambda}) \otimes \check{\pi}(\boldsymbol{\mu})),
$$

$$
\begin{gathered}
D_{p}^{\text {ord }}(\pi(\lambda) \otimes \check{\pi}(\mu)) \text { has at most a simple pole at } \Delta \text { and } \\
\operatorname{Res}_{\Delta} D_{p}^{\text {ord }}(\pi(\lambda) \otimes \check{\pi}(\mu))=\delta_{\lambda, \mu} \frac{h R_{p}}{w \sqrt{D}} \prod_{\mathfrak{q} \mid N p}\left(1-\frac{1}{N(\mathfrak{q})}\right)
\end{gathered}
$$

where $\delta_{\lambda, \mu}=\left\{\begin{array}{ll}1 & \text { if } \lambda=\mu \\ 0 & \text { otherwise, }\end{array} R_{p}\right.$ is the p-adic regulator of $F, D$ is the discriminant of $F, h$ is the class number of $F$ and $w=2$ is the number of roots of unity in $F$. As for the parity of $R_{p} / \sqrt{D}$, it is determined so that the p-adic Dedekind zeta function $\zeta_{F, p}(s)$ of $F$ satisfies

$$
\operatorname{Res}_{s=1} \zeta_{F, p}(s)=\frac{2^{[F: \mathbb{Q}]} h R_{p}}{w \sqrt{D}} \prod_{\mathfrak{P} \mid p}\left(1-\frac{1}{N(\mathfrak{P})}\right) \quad \text { (see [12]). }
$$

When $d>0\left(\Leftrightarrow R_{p}=0\right)$, we prove that $D_{p}^{\text {ord }}(\pi(\lambda) \otimes \check{\pi}(\mu))$ is entire, and therefore, the above assertion still remains true independent of the choice of $w$. To have a legitimate $L$-values at specialization at $P \otimes Q$, we need to add to $D_{p}(P, Q ; \pi(\boldsymbol{\lambda}) \otimes \check{\pi}(\boldsymbol{\mu}))$ Euler factors $\prod_{\mathfrak{P} \mid p}\left(1-\lambda_{P}(\mathbb{T}(\mathfrak{\Re})) / \mu_{Q}(\mathbb{T}(\mathfrak{P}))\right)^{-1}$, which does 
not show up in $D_{p}^{\text {ord }}(\pi(\boldsymbol{\lambda}) \otimes \check{\pi}(\boldsymbol{\mu}))$ because $V(\pi(\lambda) \otimes \check{\pi}(\mu))^{I_{\mathfrak{*}}}$ is one dimensional but $V(\pi(\boldsymbol{\lambda}) \otimes \check{\pi}(\boldsymbol{\mu}))^{I_{\mathfrak{\beta}}}=\{0\}$. Thus the residue formula (ii) actually tell us the value of the $(r-1)$-th derivative $D_{p}^{\text {ord }}(\pi(\boldsymbol{\lambda}) \otimes \check{\pi}(\boldsymbol{\mu}))$ at $P \otimes P$, where $r$ is the order of zero at $\Delta$ of $\prod_{\mathfrak{P} \mid p}(1-\lambda(\mathbb{T}(\mathfrak{B})) / \mu(\mathbb{T}(\mathfrak{B})))$. If $R_{p} \neq 0$, the value is non-zero. We now deal with the nearly ordinary case:

Conjecture 6.3.3 - Let $\lambda: h^{\text {n.ord }}\left(N p^{\infty} ; \mathcal{O}\right) \rightarrow \llbracket$ and $\mu: h^{\text {n.ord }}\left(J p^{\infty} ; \mathcal{O}\right) \rightarrow \square$ be two primitive $\Lambda$-algebra homomorphisms. Let $S=(\emptyset, I, \emptyset)$. Suppose that there exist genuine $p$-adic L-functions $L_{S, i_{\infty}}(\varphi \otimes \check{\rho})$ of type $S$ and $L_{p, i_{\infty}}(\operatorname{Ad}(\varphi))$. Then

(i) $L_{S, i_{\infty}}(\varphi \otimes \check{\rho})$ is holomorphic on $\mathscr{X}_{\mathbb{1}} \times \mathscr{L}_{\mathbb{1}}$; in other words, $L_{S, i_{\infty}}(\varphi \otimes \check{\rho}) \epsilon$ " $\widehat{\otimes}_{0} \downarrow \widehat{\otimes}_{0} \Omega$;

(ii) $D_{p}(\pi(\lambda) \otimes \check{\pi}(\mu))=\frac{L_{S, i_{\infty}}(\varphi \otimes \check{\rho})}{L_{p, i_{\infty}}(\operatorname{Ad}(\varphi))}$ up to units in \and finite Euler factors outside $p$.

In the following paragraphs, we shall give an outline of a proof of Theorem 6.3.2. Since a full detail of construction of such $L$-function is given in [41] in the nearly ordinary case, we just satisfy ourselves with a brief description of difference in the proof of the $p$-ordinary case from the nearly $p$-ordinary case.

\subsection{Auxiliary $L$-functions}

We start preparing for the proof of Theorem 6.3.2. We need to introduce several notation to describe a crude form of the $p$-adic $L$-function which we later construct. We fix two ideals $N$ and $J$ prime to $p$ in $\mathfrak{r}$, and let $L$ be the least common multiple of $N$ and $J$, that is, $L=N \cap J$. We consider two primitive $\Lambda^{\text {ord }}$-algebra homomorphisms $\lambda: h^{\text {ord }}\left(N p^{\infty} ; \mathcal{O}\right) \rightarrow \llbracket$ and $\mu: h^{\text {ord }}\left(J p^{\infty} ; \mathbb{O}\right) \rightarrow \llbracket$, where $\llbracket$ is a normal finite extension of $\mathcal{O}\left[\left[\mathbf{G}^{\text {ord }}\right]\right]$ for $\mathbf{G}^{\text {ord }}$ with respect to $\widehat{\Gamma}_{1}(N)$. By extending scalar if necessary, we

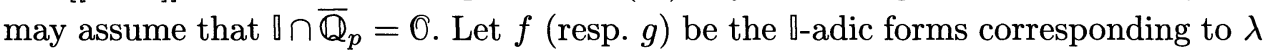
(resp. $\mu$ ) as explained in Section 2.7 of Chapter 2, and put $\varphi \in \pi(\lambda)$ and $\rho \in \pi(\mu)$. We first define certain Dirichlet series related to $L\left(s, \varphi_{P} \otimes \check{\rho}_{Q}\right)$ for positive arithmetic points $P$ and $Q$. Since $\operatorname{det}\left(\pi\left(\mu_{Q}\right)\right)=\chi_{Q} \mathcal{N}$ for the central character $\chi_{Q}$ of $g(Q)$, as is well known, we have

$$
\check{\pi}\left(\mu_{Q}\right) \cong\left(\chi_{Q} \mathcal{N}\right)^{-1} \otimes \pi\left(\mu_{Q}\right) \cong \mathcal{N}^{-[m(P)]} \otimes \pi\left(\mu_{Q}^{c}\right),
$$

where $c$ is complex conjugation and $m(P)=[m(P)] t$ for an integer $[m(P)]$ (because $n(P)=0)$. We define $\check{\mu}_{Q}(\mathbb{T}(\mathfrak{n}))=\mu_{Q}^{c}(\mathbb{T}(\mathfrak{n})) N_{F / Q}(\mathfrak{n})^{-[m(Q)]}$ and

$$
\begin{array}{r}
L\left(s, \lambda_{P} \otimes \check{\mu}_{Q}\right)=L_{L p}\left(2 s, \chi_{Q}^{-1} \psi_{P}\right) \sum_{0 \neq \mathfrak{n} \subset \mathfrak{r}} \lambda_{P}(\mathbb{T}(\mathfrak{n})) \check{\mu}_{Q}(\mathbb{T}(\mathfrak{n})) N_{F / \mathbb{Q}}(\mathfrak{n})^{-s} \\
\left.=L_{L p}\left(2 s, \chi_{Q}^{-1} \psi_{P}\right) \sum_{0 \neq \mathfrak{n} \subset \mathfrak{r}} a(\mathfrak{n}, f(P)) a\left(\mathfrak{n}, g(Q)^{c}\right)\right) N_{F / \mathbb{Q}}(\mathfrak{n})^{-[m(Q)]-s}
\end{array}
$$


where " $c$ " denotes complex conjugation and $\chi_{Q}$ (resp. $\left.\psi_{P}\right)$ is the central character of $g(Q)$ (resp. $f(P))$. Here the subscript " $L p$ " of the first factor $L_{L p}\left(2 s, \chi_{Q}^{-1} \psi_{P}\right)$ implies that this $L$-function has trivial Euler factor "1" at primes dividing $L p$. Since the last expression is given without referring to $\lambda$ or $\mu$, the definition works well for any classical modular forms $h$ and $h^{\prime}$ of weight $\kappa(P)$ and $\kappa^{\prime}(Q)$. We thus define a similar $L$-function $D\left(s, h, h^{\prime}\right)$ by

$$
D\left(s, h, h^{\prime}\right)=\sum_{0 \neq \mathfrak{n} \subset \mathfrak{r}} a(\mathfrak{n}, h) a\left(\mathfrak{n}, h^{\prime}\right) N_{F / \mathbb{Q}}(\mathfrak{n})^{-s-([m(P)]+[m(Q)]) / 2} .
$$

This zeta function looks a bit different from the one introduced in [41] (4.6), and these two zeta functions are related in the following manner:

$$
D\left(s, h, h^{\prime}\right)=D\left(s, h, h^{\prime}, \mathrm{id}\right),
$$

where the right-hand side is the zeta function defined in [41] for the identity character $\theta=$ id.

We now recall the Euler factorization of $L\left(s, \lambda_{P} \otimes \check{\mu}_{Q}\right)$ in terms of automorphic representations spanned by $f(P)$ and $g(Q)$. The following formula also holds in the nearly ordinary case. For each automorphic representation $\pi=\otimes_{\mathfrak{l}} \pi_{\mathfrak{l}}$ of $G(\mathbb{A})$, we define $\alpha_{\mathfrak{l}}(\pi), \beta_{\mathfrak{l}}(\pi) \in \mathbb{C}$ as follows: choosing a prime element $\varpi$ of $F_{\mathfrak{l}}$,

$$
\begin{aligned}
\alpha_{\mathfrak{l}}(\pi)= \begin{cases}\eta(\varpi) & \text { if } \pi_{\mathfrak{l}} \cong \pi\left(\eta, \eta^{\prime}\right) \text { with } \eta \text { unramified, } \\
\eta(\varpi) & \text { if } \pi_{\mathfrak{l}} \cong \sigma\left(\eta, \eta^{\prime}\right) \text { with } \eta \text { unramified and } \eta \eta^{\prime-1}=||_{\mathfrak{l}}^{-1}, \\
0 & \text { if } \pi_{\mathfrak{l}} \text { is super cuspidal or } \pi_{\mathfrak{l}} \cong \pi\left(\eta, \eta^{\prime}\right) \text { or } \sigma\left(\eta, \eta^{\prime}\right) \text { with } \eta \text { ramified, }\end{cases} \\
\beta_{\mathfrak{l}}(\pi)= \begin{cases}\eta^{\prime}(\varpi) & \text { if } \pi_{\mathfrak{l}} \cong \pi\left(\eta, \eta^{\prime}\right) \text { with } \eta^{\prime} \text { unramified, } \\
0 & \text { if } \pi_{\mathfrak{l}} \cong \sigma\left(\eta, \eta^{\prime}\right) \text { with } \eta \text { unramified and } \eta \eta^{\prime-1}=||_{\mathfrak{l}}^{-1}, \\
0 & \text { if } \pi_{\mathfrak{l}} \text { is super cuspidal or } \pi_{\mathfrak{l}} \cong \pi\left(\eta, \eta^{\prime}\right) \text { or } \sigma\left(\eta, \eta^{\prime}\right) \text { with } \eta^{\prime} \text { ramified. }\end{cases}
\end{aligned}
$$

Write $\pi$ (resp. $\pi^{\prime}$ ) for the automorphic representation of $G(\mathbb{A})=G L_{2}\left(F_{\mathbb{A}}\right)$ generated by right translation of $f(P)$ (resp. $g(Q)$ ). We decompose $\pi=\otimes_{\mathfrak{l}} \pi_{\mathfrak{l}}$ and the contragredient $\check{\pi}^{\prime}=\otimes_{\mathfrak{l}} \check{\pi}_{\mathfrak{l}}^{\prime}$. We put

$L_{\mathfrak{l}}(X)=\left(1-\alpha_{\mathfrak{l}}(\pi) \alpha_{\mathfrak{I}}\left(\check{\pi}^{\prime}\right) X\right)\left(1-\alpha_{\mathfrak{l}}(\pi) \beta_{\mathfrak{I}}\left(\check{\pi}^{\prime}\right) X\right)\left(1-\beta_{\mathfrak{l}}(\pi) \alpha_{\mathfrak{l}}\left(\check{\pi}^{\prime}\right) X\right)\left(1-\beta_{\mathfrak{I}}(\pi) \beta_{\mathfrak{l}}\left(\check{\pi}^{\prime}\right) X\right)$.

Then we know

$$
L\left(s, \lambda_{P} \otimes \check{\mu}_{Q}\right)=\prod_{\mathfrak{l}: \text { prime }} L_{\mathfrak{l}}\left(N(\mathfrak{l})^{-s}\right)^{-1} .
$$

Then it is easy to check (see Lemma 6.6 .1 below)

$$
L\left(s, \lambda_{P} \otimes \check{\mu}_{Q}\right)=L_{L p}\left(2 s, \psi_{P} \chi_{Q}^{-1}\right) D\left(s-1+\frac{[m(Q)-m(P)]}{2}, f(P), g(Q)^{c}\right) .
$$


In some cases, in particular if $P=Q$ and $\lambda=\mu$, the above $L$-function may not be primitive (that is, $L\left(s, \lambda_{P} \otimes \check{\mu}_{Q}\right)$ possibly differ from $L\left(s, \pi\left(\lambda_{P}\right) \otimes \check{\pi}\left(\mu_{Q}\right)\right)$ by finitely many Euler factors). It is easy to determine the missing Euler factors of $L\left(s, \lambda_{P} \otimes \check{\mu}_{Q}\right)$ comparing the definition of $L\left(s, \lambda_{P} \otimes \check{\mu}_{Q}\right)$ with that of $L\left(s, \varphi_{P} \otimes \check{\rho}_{Q}\right)$ (see (L) in Section 3.1 of Chapter 3).

For a complex modular form $h$ of weight $\kappa$ with $n(\kappa)=0$, its unitarization $h^{u}$ is defined by

$$
h^{u}(x)=\left|D_{F}\right|^{-([m(\kappa)]+1) / 2} h(x)|\operatorname{det}(x)|_{\AA}^{([m(\kappa)]-1) / 2} .
$$

The right translations of $h^{u}$ under $G(\mathbb{A})$ span a unitary automorphic representation. To describe the level of $f(P)$ and $g(Q)$ exactly, we write $S_{F}$ for the set of all prime factors $\mathfrak{P}$ of $p$ in $\mathfrak{r}$. For each $S_{F}$-tuple $\alpha=(\alpha(\mathfrak{B}))_{\mathfrak{R} \in S_{F}}$ of integers $\alpha(\mathfrak{B})$, we write $\mathfrak{P}^{\alpha}=\prod_{\mathfrak{B} \in S_{F}} \mathfrak{P}^{\alpha(\mathfrak{P})}$. Let $\kappa^{\prime}$ (resp. $\kappa$ ) be the weight of $\mu$ (resp. $\lambda$ ), that is, the restriction of $\mu$ (resp. $\lambda$ ) to $\mathbf{G}^{\text {ord }}$. For each arithmetic point $P$ of $\mathscr{L}_{\mathbb{l}}$, we write $\kappa(P)$ for the (arithmetic) weight of $P$. Then for sufficiently large $\alpha$,

$$
f(P) \in S_{\kappa(P)}^{o r d}\left(N \Re^{\alpha} ; \overline{\mathbb{Q}}\right) \quad \text { and } \quad g(Q) \in S_{\kappa(Q)}^{o r d}\left(J \Re^{\alpha} ; \overline{\mathbb{Q}}\right) .
$$

We can easily relate $g(Q)$ with the modular form $g(Q)^{c}$ whose Fourier coefficients are given by $a(y, g(Q))^{c}$. For that purpose, let $\varpi_{\mathfrak{B}}$ be the prime element in $\mathfrak{r}_{\mathfrak{B}}$ for $\mathfrak{B} \in S_{F}$ and let $n$ (resp. $j$ ) be a finite idele such that $n \mathfrak{r}=N$ (resp. $\left.j \mathfrak{r}=J\right)$ and $n_{\mathfrak{q}}=1$ (resp. $\left.j_{\mathfrak{q}}=1\right)$ outside $N$ (resp. $J$ ). Define an idele $n \varpi^{\alpha}$ so that $\left(n \varpi^{\alpha}\right)_{\mathfrak{q}}=1$ for $\mathfrak{q}$ outside $N p,\left(n \varpi^{\alpha}\right)_{\mathfrak{q}}=n_{\mathfrak{q}}$ for prime $\mathfrak{q}$ dividing $N$ and $\left(n \varpi^{\alpha}\right)_{\mathfrak{\beta}}=\varpi_{\mathfrak{B}}^{\alpha(\mathfrak{B})}$ for $\mathfrak{B} \in S_{F}$. We then define $\tau\left(n \varpi^{\alpha}\right)$ by

$$
\tau\left(n \varpi^{\alpha}\right)=\left(\begin{array}{cc}
0 & -1 \\
n \varpi^{\alpha} & 0
\end{array}\right) \in G\left(\mathbb{A}^{(\infty)}\right) .
$$

For each modular form $\phi$ in $M_{\kappa}\left(N \Re^{\alpha} ; \mathbb{C}\right)$, if $\phi$ has a central character $\xi$, we define the unitarization $\xi^{u}$ by $\xi^{u}=\xi /|\xi|$ which is of finite order. Then, we define

$$
\phi \mid \tau\left(n \varpi^{\alpha}\right)(x)=\xi^{u}(\operatorname{det}(x)) \phi\left(x \tau\left(n \varpi^{\alpha}\right)\right) \in M_{\kappa^{c}}\left(N \Re^{\alpha} ; \mathbb{C}\right),
$$

where $\kappa^{c}$ is the complex conjugation of $\kappa$. We define the complex conjugate $h^{c}$ of $h$ by $a\left(y, h^{c}\right)=a(y, h)^{c}$ for all $y$. Then $g(Q)^{c}$ is a non-zero constant multiple of $g(Q) \mid \tau\left(j \varpi^{\alpha}\right)$ if $g(Q)$ is primitive of conductor $J \mathfrak{P}^{\alpha}$ (that is, of exact level $J \mathfrak{P}^{\alpha}$ ).

\subsection{Ordinary $p$-adic Rankin products}

Let $\square \widehat{\otimes}_{\mathbb{O}} \square$ be the profinite completion of $\square \otimes_{\mathcal{O}} \square$ (that is, the $\mathfrak{m}$-adic completion of $\square \otimes \square$ for the unique maximal ideal $m$ of $\square \otimes \square)$. Under $\square \cap \overline{\mathbb{Q}}_{p}=\mathcal{O}, \square \widehat{\otimes}_{\mathbb{Q}} \square$ is an integral 
domain. For each pair of points $(P, Q)$ in $\mathscr{Y}=\mathscr{X}_{\mathbb{1}} \times \mathscr{X}_{\mathbb{1}}$, regarding $P$ and $Q$ as $\mathbb{O}$-algebra homomorphisms of $\llbracket$ into $\Omega$, we have an 0 -algebra homomorphism $P \otimes Q: \square \widehat{\otimes}_{\mathbb{O}} \square \rightarrow \Omega$. Thus for any $\Phi \in \mathbb{Q} \widehat{\rrbracket}$, we can regard $\Phi$ as a $p$-adic analytic function on $y$ with values in $\Omega$ by $\Phi(P, Q)=P \otimes Q(\Phi)$. Even if $\Phi=\Psi / \Psi^{\prime}$ is an element in the quotient field of $\llbracket \widehat{\otimes} \mathbb{d}$, we can think of $\Phi$ as a $p$-adic meromorphic function on $\mathscr{Y}$ whose value is given by $\Phi(P, Q)=\Psi(P, Q) / \Psi^{\prime}(P, Q)$ whenever we can choose a denominator $\Psi^{\prime}$ so that $\Psi^{\prime}(P, Q) \neq 0$. We first state our result in a crude form and later deduce out of it the desired $p$-adic $L$-function $D_{p}^{\text {ord }}=D_{p}^{\text {ord }}(\pi(\lambda) \otimes \check{\pi}(\mu))$.

Theorem 6.5.1 — There exists a unique element $D^{\text {ord }}$ in the quotient field of $\square \widehat{\otimes}_{\mathbb{O}} \square$

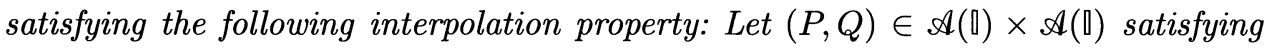
(Ad) in Section $6.1\left(\Leftrightarrow m(P)>m(Q)>0\right.$ in the ordinary case). Then $D^{\text {ord }}$ is finite at $(P, Q)$, and we have, for sufficiently large $\alpha$,

$$
\left(\chi_{Q}\right)_{\infty}(-1) D^{\text {ord }}(P, Q) N_{F / \mathbb{Q}}\left(N \mathfrak{B}^{\alpha}\right)^{([m(P)]-1) / 2} N_{F / \mathbb{Q}}\left(J \mathfrak{B}^{\alpha}\right)^{-1}
$$

$$
=\left(\chi_{Q} \psi_{P}\right)_{\infty}(-1) C_{0}(P, Q) \frac{L_{L p}\left(2, \psi_{P} \chi_{Q}^{-1}\right) D\left(\frac{[m(Q)-m(P)]}{2}, f(P), g_{\alpha}\right)}{\left(\left(f(P)^{u}\right)^{c} \mid \tau\left(n \varpi^{\alpha}\right), f(P)^{u}\right)_{\alpha}}
$$

where $(,)_{\alpha}$ denotes the Petersson inner product of level $N_{\mathfrak{P}}^{\alpha}$ (defined in [41] Section 4), $g_{\alpha}=g(Q) \mid \tau\left(j \varpi^{\alpha}\right)$ and

$C_{0}(P, Q)=|D|^{1+[m(Q)-m(P)]}(2 \pi i)^{m(P)-m(Q)} 2^{-m(P)}(2 \pi)^{-m(P)-2 t} \Gamma([m(Q)]+1)^{[F: \mathbb{Q}]}$.

Let $\mathfrak{x}$ be an ideal prime to $p L$ and $\langle\mathfrak{x}\rangle$ be the projection of the class $[\mathfrak{x}]$ of $\mathfrak{x}$ to $\mathbf{W}^{\text {ord }}$. Then, for any $H \in \llbracket$ which kills the congruence module $C_{0}(\lambda ; \rrbracket)$ for $h^{\text {ord }}\left(N p^{\infty} ; \mathcal{O}\right)$, $\left(1-\delta_{\lambda, \mu}\langle\mathfrak{x}\rangle \otimes\langle\mathfrak{x}\rangle^{-1}\right)(H \otimes 1) D^{\text {ord }}$ is integral (i.e. $\left.\left(1-\delta_{\lambda, \mu}\langle\mathfrak{x}\rangle \otimes\langle\mathfrak{x}\rangle^{-1}\right)(H \otimes 1) D^{\text {ord }} \in \mathbb{\square} \widehat{\otimes} \mathbb{0}\right)$ and we have

(b)

$$
\left\{\left(1-\langle\mathfrak{x}\rangle \otimes\langle\mathfrak{x}\rangle^{-1}\right) D^{o r d}\right\}(P, P)=-\log _{p}\left(\mathcal{N}(\langle\mathfrak{x}\rangle) \frac{h(F) R_{p}}{w \sqrt{D}} \prod_{\mathfrak{q} \mid N p}\left(1-\frac{1}{N_{F / \mathbb{Q}}(\mathfrak{q})}\right) \delta_{\lambda, \mu}\right.
$$

where $\delta_{\lambda, \mu}=1$ or 0 according as $\lambda=\mu$ or not.

In Theorem 5.1 of [41] which is the nearly $p$-ordinary counterpart of the above theorem, we formulated the result in terms of the constant $C(P, Q)$ given by $\left(\chi_{Q} \psi_{P}\right)_{\infty}(-1) C_{0}(P, Q)$. This theorem is essentially the specialization of Theorem 5.1 in [41] to the $p$-ordinary line. However, the construction of $D^{\text {ord }}$ is a bit different from that of $\mathscr{D}$ in [41], because we use the ordinary Eisenstein measure instead of the nearly ordinary Eisenstein measure employed in [41]. Since the proof of Theorem 6.5.1 is exactly parallel to that of Theorem 5.1 in [41] in this sense, we recall in details the construction of the ordinary Eisenstein measure in Section 6.7 
and its convolution along $\lambda$ in Sections 6.8 and 6.9, but the description of the proof of Theorem 6.5.1 is brief (Section 6.10).

We now try to relate $D\left(s, f(P), g_{\alpha}\right)$ with $L_{p}\left(s, \lambda_{P} \otimes \check{\mu}_{Q}\right)$. Let us introduce further notation: Let $h_{0}$ be a common eigenform of all Hecke operators corresponding to an $\mathcal{O}$-algebra homomorphism $\phi: h_{\rho}\left(N p^{\beta} ; \mathcal{O}\right) \rightarrow \mathcal{O}$ with $n(\rho)=0$. Let $h$ be the primitive form of conductor $N \mathfrak{P}^{\alpha}(0 \leq \alpha \leq \beta)$ associated with $h_{0}$ (and $\left.\phi\right)$. Write $h \mid \mathbb{T}(\mathfrak{l})=\phi^{\circ}(\mathbb{T}(\mathfrak{l})) h$. Then $\phi^{\circ}(\mathbb{T}(\mathfrak{l}))=\phi(\mathbb{T}(\mathfrak{l}))$ for almost all primes $\mathfrak{l}$. Let $L(s, \phi)=\sum_{\mathfrak{n}} \phi^{\circ}(\mathfrak{n}) N(\mathfrak{n})^{-s}$ be the standard $L$-function for $\phi$. Then we know that $h^{u} \mid \tau\left(n \varpi^{\alpha}\right)=W(\phi)\left(h^{u}\right)^{c}$ for a constant $W(\phi)$ with absolute value 1 . In fact, it is well known that (e.g. [41] (4.10)) with a suitable $\Gamma$-factor $\Gamma(s, \phi)$, we have a functional equation:

$$
\Gamma(s, \phi) L(s, \phi)=i^{-k} W(\phi)\left|d^{2} n \varpi^{\alpha}\right|_{\mathbb{A}}^{s-([k] / 2)} \Gamma(1-s, \check{\phi}) L(1-s, \check{\phi}) \quad(k=m(\rho)+t),
$$

where $\check{\phi}$ corresponds to the contragredient $\check{\pi}(\phi)$ of $\pi(\phi), d \in F_{\mathrm{A}(\infty)}^{\times}$is a finite idele giving the absolute different $d \mathfrak{x}$ and

$$
\check{\phi}(\mathbb{T}(\mathfrak{l}))=N(\mathfrak{l})^{-[m(\rho)]} \phi(\mathbb{T}(\mathfrak{l}))^{c} .
$$

Thus the root number of the functional equation is given by $i^{-k} W(\phi)$. Let $\pi=\otimes_{\mathfrak{q}} \pi_{\mathfrak{q}}$ be the automorphic representation generated by the right translations of $h_{0}$. As is well known, the constant term of the functional equation $i^{-k} W(\phi)$ can be decomposed into the product of local factors (e.g. [41] (4.10c)). To describe this, we assume that $\pi_{\mathfrak{\beta}}$ for prime factors $\mathfrak{B}$ of $p$ is either principal or special. For each quasi-character $\xi: F_{\mathfrak{B}}^{\times} \rightarrow \mathbb{C}^{\times}$, we define

$$
\varepsilon(\xi)=\xi\left(d \varpi^{r}\right) G(\xi) /\left|\xi\left(d \varpi^{r}\right) G(\xi)\right| \quad\left(G(\xi)=\sum_{u} \xi_{\bmod \varpi^{r}} \xi^{-1}(u) \mathbf{e}_{F_{\mathfrak{\beta}}}\left(u / \varpi^{r} d\right)\right),
$$

where $\mathbf{e}_{F_{\mathfrak{\beta}}}(x)=\exp \left(-2 \pi i\left[\operatorname{Tr}_{F_{\mathfrak{\beta}} / \mathbb{Q}_{p}}(x)\right]_{p}\right)$ for the $p$-fractional part $[y]_{p}$ of $y \in \mathbb{Q}_{p}, \varpi$ is a prime element of $F_{\mathfrak{R}}, \mathfrak{P}^{r}$ is the conductor of $\xi$ and $d \mathfrak{r}_{\mathfrak{R}}$ is the different of $F_{\mathfrak{P}}$ for $d \in F_{\Re}$. The appearance of $\xi^{-1}$ instead of $\xi$ in the definition of the Gauss sum $G(\xi)$ is to adjust with the classical definition. In [41], the symbol $G\left(\xi^{-1}\right)$ is used for the above $G(\xi)$. Actually for $a \in F \cap r_{\mathfrak{B}}^{\times}$and a Hecke character $\eta, 1=\eta(a)=\eta_{\mathfrak{B}}(a) \eta^{(\mathfrak{B})}(a)$ and hence $\eta^{(\mathfrak{B})}(a)=\eta_{\mathfrak{B}}(a)^{-1}$. It is common to use $a \mapsto \eta^{(\mathfrak{B})}(a)$ to define the global Gauss sum. We simply put $\varepsilon(\xi)=\xi(d) /|\xi(d)|$ if $\xi$ is unramified. Then we can decompose

$$
W(\phi)=W^{\prime}(\phi) \prod_{\mathfrak{B} \mid p} W_{\mathfrak{R}}(\phi),
$$

where

$$
\begin{aligned}
& W_{\mathfrak{B}}(\phi)=\varepsilon(\xi) \varepsilon\left(\xi^{\prime}\right) \quad \text { if either } \pi_{\mathfrak{B}}=\sigma\left(\xi, \xi^{\prime}\right) \text { with ramified } \xi \text { or } \pi\left(\xi, \xi^{\prime}\right), \\
& W_{\mathfrak{B}}(\phi)=-(\xi(\varpi) /|\xi(\varpi)|) \quad \text { if } \pi_{\mathfrak{B}}=\sigma\left(\xi, \xi^{\prime}\right) \text { with unramified } \xi .
\end{aligned}
$$


Let $P$ and $Q$ be two positive arithmetic points of $\mathscr{Q}_{\rrbracket}$. To state our result, write $\pi_{\mathfrak{B}}$ (resp. $\pi_{\mathfrak{B}}^{\prime}$ ) for the $\mathfrak{B}$-component of the automorphic representation generated by $f(P)$ (resp. $g(Q)$ ). Since $f(P)$ and $g(Q)$ are ordinary, for $\mathfrak{B} \in S_{F}, \pi_{\mathfrak{F}}$ and $\pi_{\mathfrak{B}}^{\prime}$ are either special or principal. Thus we write $\pi_{\mathfrak{\beta}}=\sigma\left(\eta_{\mathfrak{\beta}}, \eta_{\mathfrak{B}}^{\prime}\right)$ or $\pi\left(\eta_{\mathfrak{B}}, \eta_{\mathfrak{\beta}}^{\prime}\right)$ and $\pi_{\mathfrak{P}}^{\prime}=\sigma\left(\xi_{\Re}, \xi_{\mathfrak{R}}^{\prime}\right)$ or $\pi\left(\xi_{\Re}, \xi_{\mathfrak{\beta}}^{\prime}\right)$. Here we may assume that $\xi_{\Re}$ and $\eta_{\mathfrak{R}}$ are unramified and $\left|\xi_{\Re}(p)\right|_{p}=\left|\eta_{\Re}(p)\right|_{p}=1$. Then we introduce a Gauss sum factor:

$$
W_{0}(P, Q)=\frac{N(J)^{([m(Q)]+1) / 2} W^{\prime}\left(\mu_{Q}\right)}{N(N)^{([m(P)]-1) / 2} W^{\prime}\left(\lambda_{P}\right)} \prod_{\mathfrak{\beta} \in S_{F}} \frac{\xi \xi^{\prime}\left(d_{\mathfrak{\beta}}\right)\left|\eta \eta^{\prime}\left(d_{\mathfrak{B}}\right)\right| G\left(\xi_{\mathfrak{R}}^{\prime}\right)}{\eta \eta^{\prime}\left(d_{\mathfrak{B}}\right)\left|\xi \xi^{\prime}\left(d_{\mathfrak{B}}\right)\right| G\left(\eta_{\mathfrak{B}}^{\prime}\right)},
$$

where $d$ is the idele fixed in Section 2.3 of Chapter 2 such that $d \mathfrak{r}$ is the different of $F / \mathbb{Q}$. In [41] Section 5, instead of $W_{0}(P, Q)$, we used the constant $W(P, Q)=$ $\left(\chi_{Q} \psi_{P}\right)_{\infty}(-1) W_{0}(P, Q)$. We now deduce from Theorem 6.5.1 the following result, which is the ordinary version of Theorem 5.2 in [41]:

Theorem 6.5.2 - We have

$$
D^{\text {ord }}(P, Q)=W_{0}(P, Q) C_{0}(P, Q) S(P)^{-1} E^{\prime}(P, Q) \frac{L_{p}\left(1, \lambda_{P} \otimes \check{\mu}_{Q}\right)}{\left(f_{P}^{\circ}, f_{P}^{\circ}\right)_{\delta}},
$$

for all pairs of arithmetic points $(P, Q)$ satisfying $(A d)$, where $f_{P}^{\circ}$ is the primitive form of conductor $N \mathfrak{P}^{\delta}$ associated with $f(P)^{u}$, and $S(P)$ and $E^{\prime}(P, Q)$ are Euler factors at $p$ which will be described in Lemma 6.6.1 below.

\subsection{Deduction of Theorem 6.5.2 from Theorem 6.5.1}

We can deduce Theorem 6.5.2 from Theorem 6.5.1 in exactly the same manner as the deduction of [41] Theorem 5.2 from [41] Theorem 5.1. The only difference from the argument in [41] is that the Fourier $p$-coefficients of $g_{\alpha}$ in Theorem 6.5.1 does not vanish, while we removed those $p$-coefficients in [41] via the twist by the character $\theta$. Thus in our ordinary case, $\theta$ is the identity (and $\psi_{P}^{\prime}$ and $\chi_{Q}^{\prime}$ in [41] Section 5 are also trivial by the ordinarity of $f(P)$ and $g(Q))$. Anyway the computation in [41] p.349-351 is valid without any modification making all the values of $\theta$ and $\chi_{Q}^{\prime}$ to be trivial, and as in (5.6c) of [41], we may assume

$$
g(Q) \text { is of exact level } J \mathfrak{P}^{\alpha} \text {. }
$$

We now want to compute $g_{\alpha} \mid \tau\left(j \varpi^{\alpha}\right)$. This part is a bit different from the computation in [41] p.351 because $g(Q)$ has Fourier $p$-coefficients. This nonvanishing of $p$-coefficients affect the form of Lemma 5.3 [41] which is the key to the deduction. To formulate the $p$-ordinary version of Lemma 5.3 of [41], we recall some notation from [41]. Let $\chi$ be the central character of $\pi^{\prime}=\otimes_{\mathfrak{q}} \pi_{\mathfrak{q}}^{\prime}$ generated by right traslations of $g(Q)$. We consider its space $V=V\left(\pi^{\prime}\right)$ in $L_{2}(G(\mathbb{Q}) \backslash G(\mathbb{A}), \chi)$, which 
is the space of square integrable functions $f$ (modulo the center) on $G(\mathbb{Q}) \backslash G(\mathbb{A})$ with $f(g z)=\chi(z) f(g)$. Suppose that for each infinite place $\sigma \in I, \pi_{\sigma}^{\prime}$ is the discrete series representation $\sigma\left(\mu_{1}, \mu_{2}\right)$ with

$$
\mu_{1}(a)=|a|^{m_{\sigma} / 2} \quad \text { and } \quad \mu_{2}(a)=a^{m_{\sigma}-1}|a|^{m_{\sigma} / 2},
$$

where $m=m(Q)$. Let $\mathfrak{m}$ be an ideal of $\mathfrak{r}$. For any pair $\left(\xi, \xi^{\prime}\right)$ of finite order character of $(\mathfrak{r} / \mathfrak{m})^{\times}$, regarding them as characters of $\mathfrak{r}_{\mathfrak{m}}^{\times}$, we consider the subspace $V\left(\xi, \xi^{\prime}\right)=V\left(\pi^{\prime} ; \xi, \xi^{\prime} ; \mathfrak{m}\right)$ consisting of functions $\phi \in V$ satisfying the following conditions:

$$
\phi \text { is the lowest weight vector of weight } k=m(Q)+t
$$

(that is, $\phi$ corresponds to a holomorphic modular form of weight $k$ );

$$
\phi\left(x\left(\begin{array}{ll}
a & b \\
c & d
\end{array}\right)\right)=\xi\left(a_{\mathfrak{m}}\right) \xi^{\prime}\left(d_{\mathfrak{m}}\right) \phi(x) \quad \text { for all } \quad\left(\begin{array}{ll}
a & b \\
c & d
\end{array}\right) \in \widehat{\Gamma}_{0}(\mathfrak{m}),
$$

where $\widehat{\Gamma}_{0}(\mathfrak{m})=\left\{\left(\begin{array}{ll}a & b \\ c & d\end{array}\right) \in G(\widehat{\mathbb{Z}}) \mid c \in \mathfrak{m} \widehat{\mathfrak{x}}\right\}$. Since the central character of $\pi^{\prime}$ is given by $\chi, V\left(\xi, \xi^{\prime}\right)$ is trivial unless $\xi \xi^{\prime}=\chi$ on $\widehat{\mathfrak{r}}^{\times}$. Let $C$ be the conductor of $\pi^{\prime}$ in the sense of [11]. Then $V(\mathrm{id}, \chi ; C)$ is one dimensional and spanned by a unique element $f^{\circ}$ with $a\left(1, f^{\circ}\right)=1$. This form $f^{\circ}$ is called the primitive form associated with the representation $\pi^{\prime}$. We can describe the subspaces $V\left(\xi, \xi^{\prime}\right)$ locally. For that, let us write $\pi^{\prime}=\otimes_{\mathfrak{q}} \pi_{\mathfrak{q}}^{\prime}$ for a local representation $\pi_{\mathfrak{q}}^{\prime}$ at each place $\mathfrak{q}$ and take a representation space $V_{\mathfrak{q}}$ for $\pi_{\mathfrak{q}}^{\prime}$. When $\mathfrak{q}$ is finite, we define $V\left(\xi_{\mathfrak{q}}, \xi_{\mathfrak{q}}^{\prime} ; \mathfrak{m}_{\mathfrak{q}}\right)$ by the subspace of $V_{\mathfrak{q}}$ consisting of vectors $v$ satisfying $\pi_{\mathfrak{q}}^{\prime}\left(\left(\begin{array}{cc}a & b \\ c & d\end{array}\right)\right) v=\xi_{\mathfrak{q}}(a) \xi_{\mathfrak{q}}^{\prime}(d) v$ for all $\left(\begin{array}{ll}a & b \\ c & d\end{array}\right) \in \widehat{\Gamma}_{0}(\mathfrak{m})_{\mathfrak{q}}$. When $\mathfrak{q}$ is an infinite place $\sigma, V\left(\xi_{\mathfrak{q}}, \xi_{\mathfrak{q}}^{\prime} ; \mathfrak{m}_{\mathfrak{q}}\right)$ is the space of lowest weight vectors of weight $k_{\sigma}$. Then $V\left(\xi_{\mathfrak{q}}, \xi_{\mathfrak{q}}^{\prime} ; \mathfrak{m}_{\mathfrak{q}}\right)$ is one dimensional for almost all $\mathfrak{q}$ and, fixing a generator $v_{\mathfrak{q}}$ for such places, we can naturally identify

$$
V\left(\xi, \xi^{\prime} ; \mathfrak{m}\right)=\bigotimes_{\mathfrak{q}} V\left(\xi_{\mathfrak{q}}, \xi_{\mathfrak{q}}^{\prime} ; \mathfrak{m}_{\mathfrak{q}}\right)
$$

as modules over the (complex) Hecke algebra with respect to $\widehat{\Gamma}_{0}(\mathfrak{m})$.

Let $g_{Q}$ be the primitive form associated with $V=V\left(\pi^{\prime}\right)$. We want to express the special value:

$$
D\left(\frac{[m(Q)-m(P)]}{2}, f(P), g_{\alpha}\right)
$$

by means of $L\left(s, \lambda_{P} \otimes \check{\mu}_{Q}\right)$ (which is equal to $L\left(s, \pi \times \check{\pi}^{\prime}\right)$ up to finitely many Euler factors). By definition, $g(Q)$ belongs to $V\left(\pi^{\prime} ;\right.$ id, $\left.\chi ; J \mathfrak{P}^{\alpha}\right)$. It is known that $\pi_{\mathfrak{\beta}}^{\prime}$ for $\mathfrak{B} \in S_{F}$ is either principal or special, and the special representation occurs only when $m_{\sigma}=1$ for all the $p$-adic places $\sigma: F \hookrightarrow \overline{\mathbb{Q}} \hookrightarrow \overline{\mathbb{Q}}_{p}$ (see [34] Lemma 12.2, [38] Proposition 2.1 and Corollary 2.2). Write $\pi_{\mathfrak{\beta}}^{\prime}=\pi\left(\xi_{\mathfrak{\beta}}, \xi_{\mathfrak{\beta}}^{\prime}\right)\left(\right.$ resp. $\sigma\left(\xi_{\mathfrak{\beta}}, \xi_{\mathfrak{\beta}}^{\prime}\right)$ with 
$\xi_{\mathfrak{\beta}} \xi_{\mathfrak{\beta}}^{\prime-1}=||_{p}$ ) when $\pi_{\mathfrak{\beta}}^{\prime}$ is principal (resp. special). Here, we follow the convention explained below [38] Proposition 2.1 to describe principal series representations and

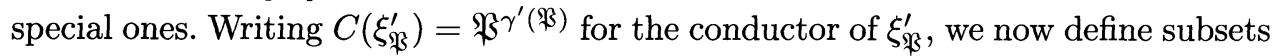
of $S_{F}$ as follows:

$$
\begin{aligned}
& \Sigma_{1}=\left\{\mathfrak{P} \in S_{F} \mid \gamma^{\prime}(\mathfrak{P})>0\right\} \\
& \Sigma_{3}=\left\{\mathfrak{P} \in S_{F} \mid \gamma^{\prime}(\mathfrak{P})=0 \text { and } \pi_{\mathfrak{P}}^{\prime} \text { is principal }\right\} \\
& \Sigma_{4}=\left\{\mathfrak{P} \in S_{F} \mid \gamma^{\prime}(\mathfrak{P})=0 \text { and } \pi_{\mathfrak{P}}^{\prime} \text { is special }\right\} .
\end{aligned}
$$

Here we use the same index system as in [41] Lemma 5.3 in order to facilitate comparison with the lemma there. Especially, in our $p$-ordinary case, the set $\Sigma_{2}$ in [41] will not show up. By [38] Corollary 2.2, we may assume that $\xi_{\Re}$ (resp. $\left.\xi_{\mathfrak{B}}^{\prime}\right)$ coincides with the trivial character on $\mathfrak{r}_{\mathfrak{R}}^{\times}\left(\right.$resp. $\left.\chi_{\mathfrak{B}}\right)$ and that $g(Q) \mid \mathbb{T}(\mathfrak{R})=$ $\xi_{\mathfrak{R}}\left(\varpi_{\mathfrak{P}}\right) g(Q)$ for the Hecke operator $\mathbb{T}(\mathfrak{P})$.

Similarly as above, let $\pi=\otimes_{\mathfrak{q}} \pi_{\mathfrak{q}}$ be the automorphic representation spanned by $f(P)$ and write its $p$-component as $\pi\left(\eta_{\mathfrak{B}}, \eta_{\mathfrak{R}}^{\prime}\right)$ or $\sigma\left(\eta_{\mathfrak{B}}, \eta_{\mathfrak{B}}^{\prime}\right)$ for $\mathfrak{R} \in S_{F}$ so that $f(P) \mid \mathbb{T}(\mathfrak{B})=\eta\left(\varpi_{\mathfrak{B}}\right) f(P)$ (where $\eta_{\mathfrak{P}}$ is unramified at $\mathfrak{\Re} \in S_{F}$ ). We define

$$
\begin{aligned}
& \Sigma_{1}(P)=\left\{\mathfrak{P} \in S_{F} \mid \eta_{\mathfrak{B}}^{\prime} \text { is ramified }\right\} \\
& \Sigma_{2}(P)=\left\{\mathfrak{P} \in S_{F} \mid \pi_{\mathfrak{B}} \text { is principal }\right\} \\
& \Sigma_{3}(P)=\left\{\mathfrak{P} \in S_{F} \mid \pi_{\mathfrak{B}} \text { is special }\right\} .
\end{aligned}
$$

Then Theorem 6.5.2 is a direct consequence of Theorem 6.5.1 and the following result:

\section{Lemma 6.6.1 - (i) We have}

$$
\alpha(\mathfrak{P})= \begin{cases}1 & \text { when } \mathfrak{P} \in \Sigma_{3} \cup \Sigma_{4} \\ \gamma^{\prime}(\mathfrak{B}) & \text { otherwise, }\end{cases}
$$

where $J \mathfrak{B}^{\alpha}$ is the exact level of $g(Q)$ and $\alpha=(\alpha(\mathfrak{B}))_{\mathfrak{B} \in S_{F}}$.

(ii) Write e for $[m(Q)]-1$ and define operators $B_{\mathfrak{P}}$ for each $\mathfrak{B} \in S_{F}$ acting on $V\left(\pi^{\prime \prime} ; \mathrm{id}, \chi_{Q}^{-1} ; J \mathfrak{B}^{\alpha}\right)$ for the unitarization $\pi^{\prime \prime}$ of the contragredient of $\pi^{\prime}$ by

$$
\begin{aligned}
& B_{\mathfrak{R}}=\mathrm{Id} \quad \text { if } \mathfrak{P} \in \Sigma_{1} \cup \Sigma_{4}, \\
& B_{\mathfrak{P}}=\mathrm{Id}-\left|\varpi_{\mathfrak{R}}\right|_{p}^{-(e / 2)-1} \xi^{\prime-1}\left(\varpi_{\mathfrak{R}}\right)\left[\varpi_{\mathfrak{P}}\right]_{0} \quad \text { if } \mathfrak{R} \in \Sigma_{3} .
\end{aligned}
$$

Then we have $\left\{g(Q) \mid \tau\left(j \varpi^{\alpha}\right)\right\}^{u}=C_{0}\left(g(Q)^{c}\right)^{u} \mid B$ for $B=\prod_{\mathfrak{B} \in S_{F}} B_{\Re}$, where

$$
C_{0}=W\left(\mu_{Q}\right)\left|j \varpi^{\alpha}\right|_{\mathbb{A}}^{-e / 2} \prod_{\mathfrak{B} \in \Sigma_{3}}\left(-\xi^{\prime}\left(\varpi_{\mathfrak{P}}\right)\right)\left|\varpi_{\mathfrak{B}}\right|_{\mathfrak{B}}^{(e / 2)+1}
$$

and $h \mid\left[\varpi_{\mathfrak{\beta}}^{r}\right]_{0}(x)=h\left(x\left(\begin{array}{cc}\varpi_{\mathfrak{\beta}}^{-r} & 0 \\ 0 & 1\end{array}\right)\right)$ for $h \in V\left(\pi^{\prime \prime} ; \mathrm{id}, \chi_{Q}^{-1} ; J \mathfrak{P}^{\alpha}\right)$. 
(iii) Define an Euler factor $E_{\mathfrak{B}}^{\prime}(P, Q)$ at each $\mathfrak{P} \in S_{F}$ as follows:

$$
\begin{aligned}
& \frac{\left(1-\xi^{\prime} \eta^{-1}(\varpi)\right)}{\left(1-\xi^{-1} \eta(\varpi)|\varpi|_{\mathfrak{B}}\right)\left(1-\xi^{\prime-1} \eta(\varpi)|\varpi|_{\mathfrak{B}}\right)} \quad \text { if } \mathfrak{R} \in \Sigma_{3}, \\
& \xi^{\prime} \eta^{-1}\left(\varpi^{\gamma^{\prime}(\mathfrak{B})} \frac{1}{\left(1-\xi^{-1} \eta(\varpi)|\varpi|_{\mathfrak{B}}\right)} \quad \text { if } \mathfrak{B} \in \Sigma_{1},\right. \\
& -\xi \eta^{-1}(\varpi) \frac{1}{\left(1-\xi^{-1} \eta(\varpi)|\varpi|_{\mathfrak{B}}^{2}\right)} \quad \text { if } \mathfrak{R} \in \Sigma_{4},
\end{aligned}
$$

where we have written $\varpi$ for $\varpi_{\mathfrak{\beta}}$. Then the Euler factor at $\mathfrak{P}$ of

$$
L_{L p}\left(2, \chi_{Q}^{-1} \psi_{P}\right) D\left(\frac{[m(Q)-m(P)]}{2}, f(P), g(Q) \mid \tau\left(j \varpi^{\alpha}\right)\right)
$$

is given by $C_{1} E(P, Q)$, where $E^{\prime}(P, Q)=\prod_{\mathfrak{B} \in S_{F}} E_{\mathfrak{\beta}}^{\prime}(P, Q)$ and

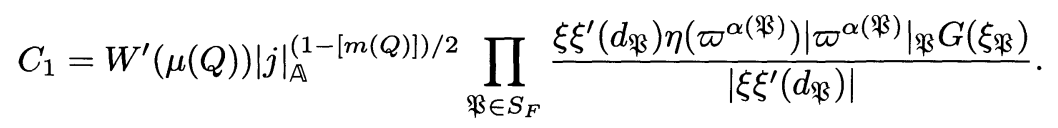

(iv) Write $f_{P}^{\circ}$ for the primitive form of the unitarization of $\pi$ and $C(\pi)=N \mathfrak{\beta}^{\delta}$ and define for $s=\#\left(\Sigma_{3}(P)\right)$

$$
\begin{aligned}
S(P)=(-1)^{s} \prod_{\mathfrak{B} \in \Sigma_{2}(P)}\left(1-\eta^{-1} \eta^{\prime}\left(\varpi_{\mathfrak{B}}\right)\left|\varpi_{\mathfrak{B}}\right|_{\mathfrak{R}}\right)\left(1-\eta^{-1} \eta^{\prime}\left(\varpi_{\mathfrak{R}}\right)\right) & \\
& \times \prod_{\mathfrak{B} \in \Sigma_{1}(P)} \eta^{\prime} \eta^{-1}\left(\varpi_{\mathfrak{R}}^{\delta(\mathfrak{R})}\right)\left|\varpi_{\mathfrak{B}}^{\delta(\mathfrak{B})}\right|_{\mathfrak{R}} .
\end{aligned}
$$

Then we have

$$
\begin{aligned}
& \frac{\left(\left(f(P)^{c}\right)^{u} \mid \tau\left(n \varpi^{\alpha}\right), f(P)^{u}\right)_{\alpha}}{\left(f_{P}^{\circ}, f_{P}^{\circ}\right)_{\delta}} \\
& =\left|\varpi^{\alpha}\right|_{\mathbb{A}}^{([m(P)]-1) / 2} \eta\left(\varpi^{\alpha}\right) \psi_{P, \infty}(-1) W^{\prime}\left(\lambda_{P}\right) S(P) \prod_{\mathfrak{B} \in \Sigma_{1}(P)} G\left(\eta^{\prime}\right) \prod_{\mathfrak{B} \in S_{F}} \frac{\eta \eta^{\prime}\left(d_{\mathfrak{\beta}}\right)}{\left|\eta \eta^{\prime}\left(d_{\mathfrak{B}}\right)\right|}
\end{aligned}
$$

The assertions (i) and (iv) follows from [41] Lemma 5.3 (ii), (vi), and the assertion (ii) follows from [41] (5.8). Since the proof (iii) is a straightforward computation similar to (and simpler than) that of Lemma 5.3 (iv) of [41], we do not repeat it here and leave it to the attentive reader. From this lemma, we conclude the assertion of Theorem 6.5.2 in the same manner as in [41] Section 5. In the sense of Conjecture 4.2 .1 (iii) in Chapter 4, the Euler factor $E^{\prime}(P, Q)$ as above is associated to $L_{S, i_{\infty}}(\pi(\lambda) \otimes \check{\pi}(\mu))$, and $S(P)$ is associated to $L_{p, i_{\infty}}(A d(\pi(\lambda)))$. This fact supports the description (iii) of Conjecture 6.3.1 and (ii) of Conjecture 6.3.3. 


\section{7 $p$-Ordinary adelic Eisenstein measure}

Here we give a construction of the ordinary Eisenstein measure. Let

$$
U_{F}\left(L p^{\alpha}\right)=\left\{x \in \widehat{\mathfrak{r}}^{\times} \mid x \equiv 1 \quad \bmod L p^{\alpha} \widehat{\mathfrak{r}}\right\} \quad \text { and } \quad U_{F}\left(L p^{\infty}\right)=\bigcap_{\alpha>0} U_{F}\left(L p^{\alpha}\right) .
$$

We consider the ray class groups $Z$ and $\bar{Z}$ defined as follows:

$$
\bar{Z}=\bar{Z}(L)=F_{\AA}^{\times} / \overline{F^{\times} U_{F}\left(L p^{\infty}\right) F_{\infty}^{\times}} \quad \text { and } \quad Z=Z(L)=F_{\AA}^{\times} / \overline{F^{\times} U_{F}\left(L p^{\infty}\right) F_{\infty+}^{\times}},
$$

where $\overline{F^{\times} U_{F}\left(L p^{\infty}\right) F_{\infty}^{\times}}$is the topological closure of $F^{\times} U_{F}\left(L p^{\infty}\right) F_{\infty}^{\times}$in $F_{A}^{\times}, F_{\infty}=$ $F \otimes_{\mathbb{Q}} \mathbb{R}$ and $F_{\infty+}^{\times}$is the identity connected component of $F_{\infty}^{\times}$. The group $\mathbf{W}^{\text {ord }}$ can be naturally regarded as an open subgroup of $\bar{Z}$ and $Z$. By class field theory, we can identify $Z$ and $\bar{Z}$ with the Galois group of a suitable abelian extension of $F$. Write $C l_{F}^{0}\left(L p^{\alpha}\right)$ (resp. $C l_{F}\left(L p^{\alpha}\right)$ ) for the (resp. narrow) ray class group modulo $L p^{\alpha}$. Then

$$
\bar{Z} \cong \lim _{\alpha} C l_{F}^{0}\left(L p^{\alpha}\right) \text { and } Z \cong \lim _{\alpha} C l_{F}\left(L p^{\alpha}\right) .
$$

By considering the class of an ideal a prime to $L p$ in every $C l_{F}\left(L p^{\alpha}\right)$ or $C l_{F}^{0}\left(N p^{\alpha}\right)$, we can associate to $\mathfrak{a}$ an element $[\mathfrak{a}]$ of $Z$ or $\bar{Z}$. Let $\omega$ be the Teichmüller character of $Z$, that is, for each ideal $\mathfrak{a}, \omega([\mathfrak{a}])=\lim _{n \rightarrow \infty} N_{F / \mathbb{Q}}(\mathfrak{a})^{p^{n}}$, where the limit is taken in $\mathbb{Z}_{p}$. Let $\mathcal{N}: Z \rightarrow \mathbb{Z}_{p}^{\times}$be the cyclotomic character, that is, $\mathcal{N}([\mathfrak{a}])=N_{F / \mathbb{Q}}(\mathfrak{a})$. We consider $\omega$ to have values in $\overline{\mathbb{Q}}^{\times}$via $i_{p}$. Thus we may think $\varepsilon \mathcal{N}^{k}$ as a character with values in $\overline{\mathbb{Q}}_{p}^{\times}$for any finite order characters $\varepsilon: Z(L) \rightarrow \overline{\mathbb{Q}}^{\times}$. Then, by a result of Deligne and Ribet [17], for any given ideal $\mathfrak{x}$ prime to $N p$, there exists a unique $p$-adic measure $\zeta=\zeta_{\mathfrak{x}, L}$ (in the sense of Mazur) such that

$$
\int_{\bar{Z}} \varepsilon \mathcal{N}^{m}(z) d \zeta_{\mathfrak{x}, L}(z)=\left(1-\varepsilon \mathcal{N}^{m}([\mathfrak{x}])\right) L_{L p}(1-m, \varepsilon)
$$

for all $m>0$ and all character $\varepsilon \mathcal{N}^{m}: \bar{Z}(L) \rightarrow \overline{\mathbb{Q}}_{p}^{\times}$. Here we write

$$
L_{L p}(s, \varepsilon)=\prod_{\mathfrak{q} \mid L p}\left(1-\varepsilon(\mathfrak{q}) N_{F / \mathbb{Q}}(\mathfrak{q})^{-s}\right) L(s, \varepsilon)
$$

where $\mathfrak{P}$ runs over all prime factors of $p$ in $\mathfrak{r}$. We write $\mathscr{C}\left(C l_{F}^{0}\left(L p^{\alpha}\right) ; \mathcal{O}\right)$ for the space of all functions on the finite group $C l_{F}^{0}\left(L p^{\alpha}\right)$, which is naturally the 0 -dual space of the group algebra $\mathcal{O}\left[C l_{F}^{0}\left(L p^{\alpha}\right)\right]$ by the pairing $\langle$,$\rangle given by \langle\xi, \phi\rangle=\sum_{[\mathfrak{a}]} \xi_{[\mathfrak{a}]} \phi([\mathfrak{a}]) \in$ 0 . Here $\xi_{[\mathfrak{a}]}$ is the coefficient of $\xi$ at $[\mathfrak{a}] \in C l_{F}^{0}\left(L p^{\alpha}\right)$. Moreover these pairings for varying $\alpha$ are compatible with the transition maps of the natural projective system $\left\{C l_{F}^{0}\left(L p^{\alpha}\right)\right\}_{\alpha}$ and the injective system $\left\{\mathscr{C}\left(C l_{F}^{0}\left(L p^{\alpha}\right) ; \mathcal{O}\right)\right\}_{\alpha}$. Thus the continuous group algebra $\mathcal{O}[[\bar{Z}]]$ can be considered as the space of $\mathcal{O}$-valued measures on $\bar{Z}$. 
Then, writing $\mathscr{C}(T ; \mathcal{O})$ for the space of continuous functions on a topological space $T$ having values in $\mathbb{O}$, we know that $\mathbb{O}[[\bar{Z}]]$ is the $\mathbb{O}$-dual of $\mathscr{C}(\bar{Z} ; \mathcal{O})$. Thus $\zeta_{\mathfrak{r}, L} \in \mathbb{O}[[\bar{Z}]]$. We regard $1-[\mathfrak{x}]$ as an element $\mu$ of $\mathbb{O}[[\bar{Z}]]$. We have $\int_{\bar{Z}} \phi(z) d \mu(z)=1-\phi([\mathfrak{x}])$.

Now we define the Eisenstein measure $E_{\mathfrak{x}, L}: \mathscr{C}(\bar{Z}(L) ; \mathcal{O}) \rightarrow M^{\text {ord }}\left(L p^{\infty} ; \mathcal{O}\right.$ ) (for each $k \geq 1$ ) by the following formulas:

$$
\begin{aligned}
\mathbf{a}_{0, p}\left(y, E_{\mathfrak{x}, L}(\phi \mathcal{N})\right) & =2^{-[F: \mathbb{Q}]} \int_{\bar{Z}} \phi \mathcal{N}(z) d \zeta_{\mathfrak{L}, L}(z), \\
\mathbf{a}_{p}\left(y, E_{\mathfrak{x}, L}(\phi \mathcal{N})\right) & =\sum_{\mathfrak{b} \supset y \mathfrak{r}}\left(\phi([\mathfrak{b}])-N_{F / \mathbb{Q}}(\mathfrak{x}) \phi([\mathfrak{x} \mathfrak{b}])\right) .
\end{aligned}
$$

To compute the $q$-expansion of $E_{\mathfrak{x}, L}(\phi \mathcal{N})$ when $\phi \mathcal{N}: \bar{Z}(L) \rightarrow \overline{\mathbb{Q}}_{p}^{\times}$is a product $\varepsilon \mathcal{N}^{k}$ for a finite order character $\varepsilon: C l_{F}\left(L p^{\alpha}\right) \rightarrow \overline{\mathbb{Q}}^{\times}$, we introduce some classical Eisenstein series. Write simply $\xi$ for $\varepsilon \mathcal{N}^{k}(k=[m(\kappa)]+1)$. Then there exists a classical modular form $E(\xi) \in M_{\kappa}\left(L p^{\alpha} ; \mathbb{C}\right)$ for $\kappa=\left(\varepsilon \mathcal{N}^{k-1}, 0\right)$ such that

$$
\mathbf{a}_{p}(y, E(\xi))=\sum_{\mathfrak{b} \supset y \mathfrak{r}} \xi \mathcal{N}^{-1}([\mathfrak{b}]) \quad \text { and } \quad \mathbf{a}_{0, p}(y \mathfrak{r}, E(\xi))=2^{-[F: \mathbb{Q}]} L_{L p}(1-k, \varepsilon)
$$

where $\varepsilon_{\kappa}(a, d)=\varepsilon(d), n(\kappa)=0$ and $m(\kappa)=k t-t$. Then we can easily check that $E(\xi) \mid \mathbb{T}(p)=E(\xi)$, and hence $E(\xi)$ is $p$-ordinary. Further we get by definition

$$
E_{\mathfrak{x}, L}(\xi)=E(\xi) \mid\left(1-\langle\mathfrak{x}\rangle \mathcal{N}([\mathfrak{x}])^{2}\right) .
$$

Thus, for any locally constant function $\phi$ on $\bar{Z}$ with values in $K, E_{\mathfrak{x}, L}(\phi \xi)$ is an element of $M_{\kappa}^{\text {ord }}\left(L p^{\infty} ; K\right)$. Since the functionals: $\phi \mapsto \mathbf{a}_{p}\left(y, E_{\mathfrak{x}, L}(\phi \xi)\right)$ and $\mathbf{a}_{0, p}\left(y, E_{\mathfrak{x}, L}(\phi \xi)\right)$ are bounded measures with values in $\mathcal{O}$ on $\mathscr{C}(\bar{Z} ; \mathcal{O})$, the linear map defined on the space $\mathscr{L} \mathscr{C}(\bar{Z} ; \mathcal{O})$ of locally constant functions $E_{\mathfrak{r}, L}: \mathscr{L} \mathscr{C}(\bar{Z} ; \mathcal{O}) \rightarrow$ $M_{\kappa}^{\text {ord }}\left(N p^{\infty} ; \mathcal{O}\right)$ extends by continuity to a $p$-adic measure having values in the space of $p$-adic modular forms $M^{\text {ord }}\left(N p^{\infty} ; \mathcal{O}\right)$. We have, by [12],

$$
E_{\mathfrak{x}, L}(i d)=-\log _{p}(\mathcal{N}(\langle\mathfrak{x}\rangle)) \frac{R_{p} h(F)}{w} \prod_{\mathfrak{q} \mid p L}\left(1-\frac{1}{N_{F / \mathbb{Q}}(\mathfrak{q})}\right) .
$$

\subsection{Convoluted measure}

Let us simply write the Eisenstein measure as $E=E_{\mathfrak{x}, L}$. Note that $\mathbf{G}^{\text {ord }}$ can be naturally considered to be an open subgroup of $Z(L)$. Then through the action of the center of $G$, we can extend the action of $\mathbf{G}^{\text {ord }}$ on $U^{*}=M\left(L p^{\infty} ; \mathbb{O}\right)$ and $S=S\left(L p^{\infty} ; \mathcal{O}\right)$ to $Z(L)$ which actually factors through $\bar{Z}(L)$ because units in $\mathfrak{r}^{\times}$ acts trivially on modular forms. For each $\mathbb{O}$-module $M$, we write $M^{*}$ for the $\mathbb{O}$-dual of $M$. Let $\varphi: M^{*} \rightarrow S\left(L p^{\infty} ; \mathcal{O}\right)$ be an $\mathbb{O}[[\bar{Z}(L)]]$-linear map for an $\mathcal{O}[[\bar{Z}(L)]]$-module 
$M$. In this paragraph, in order to give a proof of Theorem 6.5.1 in the following paragraph Section 6.10, we describe the theory of the convolution measure of $E$ and $\varphi$ developed in [41] Section 9. We can decompose $\bar{Z}(L)=\mathbb{W} \times \Delta(L)$ for a finite group $\Delta=\Delta(L)$ and the torsion-free subgroup $\mathbb{W}$. We write $\boldsymbol{\Lambda}$ for $\mathcal{O}[[\mathbb{W}]]$. We restate Lemma 9.1 of [41] which is a key to our construction:

Lemma 6.8.1 - Suppose that $M$ is a $\mathbf{\Lambda}$-submodule of a $\boldsymbol{\Lambda}$-free module of finite rank. Then $M$ satisfies the following conditions:

$$
M \cong \lim _{\alpha} M_{\alpha} \text { as } \boldsymbol{\Lambda} \text {-modules; }
$$

$M_{\alpha}$ is a $\mathbf{\Lambda}$-module and is $\mathbf{0}$-free of finite rank;

The transition maps: $M_{\beta} \rightarrow M_{\alpha}$ are all surjective.

Moreover if a $\boldsymbol{\Lambda}$-module $M$ satisfies (i), (ii) and (iii), we have

$$
\begin{aligned}
M^{*} & =\operatorname{Hom}_{\mathcal{O}}(M, \mathcal{O}) \cong \lim _{\alpha}\left(\left(\lim _{\beta} M_{\beta}^{*}\right) \otimes_{\mathcal{O}} \mathcal{O} / p^{\alpha} \mathcal{O}\right), \\
\left(M^{*}\right)^{*} & \cong M, \quad \operatorname{Hom}_{\boldsymbol{\Lambda}}\left(M, \boldsymbol{\Lambda}^{*}\right) \cong M^{*} \text { and } \operatorname{Hom}_{\boldsymbol{\Lambda}}\left(M^{*}, L^{*}\right) \cong M .
\end{aligned}
$$

Note that $U=\left(U^{*}\right)^{*}$ and $S^{*}$ satisfy the condition (i), (ii) and (iii) of Lemma 6.8.1.

We have a product $m^{\prime}: U^{*} \times S \rightarrow|D| S$ induced by the multiplication in the ring of $q$-expansions (see Section 2.4 in Chapter 2). We may define a bilinear map $m: U^{*} \times S \rightarrow S$ by $m(f, g)=|D|^{-1} m^{\prime}(f, g)$. Then by definition, we see for $z \in Z$

$$
m(f|\langle z\rangle, g|\langle z\rangle)=m(f, g) \mid\langle z\rangle .
$$

Note that $E: \mathscr{C}(\bar{Z}(L) ; \mathcal{O}) \rightarrow U^{*}$ is $\mathbb{O}[[\bar{Z}(L)]]$-linear under the action:

$$
\left.\phi \mapsto \phi\right|_{2} z\left(z^{\prime}\right)=\phi\left(z^{\prime} z\right) \mathcal{N}\left(z^{\prime}\right)^{2} \quad \text { on } \quad \mathscr{C}(\bar{Z}(L) ; \mathcal{O})
$$

Consider a compact $\mathcal{O}[[\bar{Z}(J)]]$-module $M$ satisfying the conditions (i)-(iii) of Lemma 6.8.1. We regard $M$ as an $\mathcal{O}[[\bar{Z}(L)]]$-module via a natural projection of $\bar{Z}(L)$ to $\bar{Z}(J)$. For an $\mathcal{O}[[\bar{Z}(L)]]$-linear map $\varphi: M^{*} \rightarrow S$, define

$$
\widehat{\varphi}: M^{*} \widehat{\otimes}_{0} U^{*} \rightarrow S \text { by } \widehat{\varphi}=m \circ(\varphi \otimes \mathrm{Id}),
$$

where $M^{*} \widehat{\otimes}_{\mathcal{O}} U^{*}$ is a $p$-adic completion $\lim _{j}\left(M^{*} \otimes U^{*}\right) / p^{j}\left(M^{*} \otimes U^{*}\right)$. By construction,

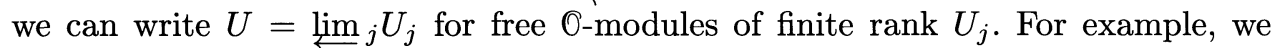
could take $U_{j}$ to be the $\mathcal{O}$-dual of $\left\{\sum_{0 \leq \kappa \leq(j t, 0)} M_{\kappa}\left(L p^{j} ; K\right)\right\} \cap M\left(L p^{\infty} ; \mathcal{O}\right)$. We write $M \widehat{\otimes}_{\mathcal{O}} U$ for the profinite completion $\varliminf_{i, j} M_{i} \otimes U_{j}$. We say that a function $\phi: M \rightarrow U^{*}$ is continuous if it is continuous under the $p$-adic topology on $U^{*}$ and under the topology of the profinite group $M$. Thus if $\phi$ is $\mathcal{C}$-linear, then $\phi$ is continuous if and only if there exists $i>0$ for any $j>0$ such that $\phi$ $\bmod p^{j}: M / p^{j} M \rightarrow U^{*} / p^{j} U^{*}$ factors through $M_{i} / p^{j} M_{i}$, where $M=\lim _{i} M_{i}$ as in 
Lemma 6.8.1. Since $U=\lim _{k} U_{k}$ for $\mathcal{O}$-free modules $U_{k}$ of finite rank satisfying the condition of Lemma 6.8.1, the image of $\phi \bmod p^{j}$ is actually contained in $U_{k}^{*} / p^{j} U_{k}^{*}$ for some $k$. We denote by $\operatorname{Hom}_{c}\left(M, U^{*}\right)$ the space of all continuous $\mathcal{O}$-linear maps. Then we have

$$
\begin{aligned}
& \operatorname{Hom}_{c}\left(M, U^{*}\right)=\lim _{j} \varliminf_{\lim _{i}} \varliminf_{k} \operatorname{Hom}_{\mathcal{O}}\left(M_{i} / p^{j} M_{i}, U_{k}^{*} / p^{j} U_{k}^{*}\right) \\
& =\lim _{j} \lim _{i, k} \operatorname{Hom}_{\mathscr{O}}\left(M_{i} / p^{j} M_{i} \otimes_{\mathcal{O}} U_{k}, \mathcal{O} / p^{j} \mathcal{O}\right)=\left(M \widehat{\otimes}_{\mathcal{O}} U\right)^{*},
\end{aligned}
$$

where $M \widehat{\otimes}_{\odot} U$ is the profinite completion of $M \otimes_{\odot} U$. As shown in [41] Lemma 9.2, we have

$$
M^{*} \widehat{\otimes}_{\mathcal{O}} U^{*} \cong \operatorname{Hom}_{c}\left(M, U^{*}\right) \cong\left(M \widehat{\otimes}_{\mathcal{O}} U\right)^{*},
$$

where the identification is given by $\phi \otimes u^{*} \mapsto\left(\phi \otimes u^{*}\right)(m)=\phi(m) u^{*}$. For each continuous function $\Phi \in \mathscr{C}(M \times \bar{Z} ; \mathcal{O})$, we define an action of $\bar{Z}(L)$ by $(\Phi \mid z)\left(m, z^{\prime}\right)=\Phi\left(z^{-1} m, z z^{\prime}\right)$. We define $E_{*}: \mathscr{C}(M \times \bar{Z} ; \mathcal{O}) \rightarrow \mathscr{C}\left(M, U^{*}\right)$ by

$$
E_{*}(\Phi)(m)=\int_{\mathbf{G}(L)}(\Phi \mid z)(m, 1) d E(z) .
$$

Then $E_{*}$ induces on $M^{*} \widehat{\otimes}_{\mathcal{O}} \mathscr{C}(\mathbf{G} ; \mathcal{O})\left(\cong \operatorname{Hom}_{c}(M, \mathscr{C}(\mathbf{G} ; \mathcal{O}))\right)$ a morphism into $\operatorname{Hom}_{c}\left(M, U^{*}\right)\left(\cong M^{*} \widehat{\otimes}_{\mathcal{O}} U^{*}\right)$. Then we have

$$
E_{*}(\Phi)(m)=\int_{\mathbf{G}(L)} \Phi\left(z^{-1} m\right)(z) d E(z) \quad \text { for } \quad \Phi \in \operatorname{Hom}_{c}(M, \mathscr{G}(\bar{Z} ; \mathfrak{O})) .
$$

We now define the convoluted measure $E * \varphi: M^{*} \widehat{\otimes}_{\mathcal{O}} \mathscr{C}(\bar{Z} ; \mathcal{O}) \rightarrow S$ by

$$
E * \varphi(\Phi)=\widehat{\varphi}\left(E_{*}(\Phi)\right)
$$

By our construction, if we let $z \in \bar{Z}(L)$ act on $\Phi$ via $\Phi \| z(m)(x)=\Phi(m)(z x) \mathcal{N}(z)^{2}$ regarding $\Phi$ as an element of $\operatorname{Hom}_{c}(M, \mathscr{C}(\bar{Z} ; \mathfrak{O}))$, then it is easy to see

$$
E * \varphi \text { is a morphism of } \mathcal{O}[[\bar{Z}]]-\text { modules. }
$$

\subsection{Convolution along $\lambda$}

We fix decomposition: $\bar{Z}(N)=\mathbb{W} \times \Delta(N)$ so that the projection $\bar{Z}(L) \rightarrow \bar{Z}(N)$ induces the identity on $\mathbb{W}$. We take $\lambda: h^{\text {ord }}\left(N p^{\infty} ; \mathcal{O}\right) \rightarrow \mathbb{Q}$ as in Theorem 6.3.2. Note here $\lambda$ factors through $h^{\text {ord }}(\psi)$ defined in Section 2.9 of Chapter 2. It is easy to check

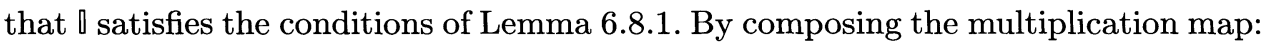
$\square \otimes_{\boldsymbol{\Lambda}} \rrbracket \rightarrow \square$ with $\lambda \otimes \mathrm{Id}$, we can naturally extend $\lambda$ to an -algebra homomorphism of

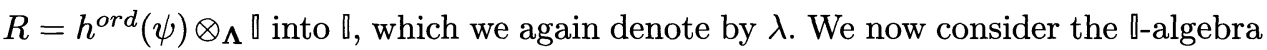
decomposition $R \otimes_{\mathbb{R}} \mathbb{K}=\mathbb{K} \oplus X$ introduced in Section 2.9 of Chapter 2 and write $1_{\lambda}$ for 


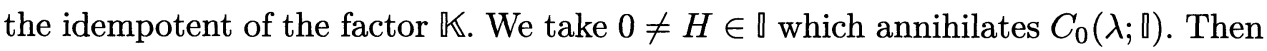
$H 1_{\lambda} \in R$. We write $S^{\text {ord }}(\psi)$ (resp. $\left.S^{\text {ord }}\right)$ for the dual of $h^{\text {ord }}(\psi)\left(\operatorname{resp} . h^{\text {ord }}\left(N p^{\infty} ; \mathbb{O}\right)\right)$ in $S$ (see Section 2.9 in Chapter 2). We also write $\check{M}=\operatorname{Hom}_{\boldsymbol{\Lambda}}(M, \boldsymbol{\Lambda})$ for any $\boldsymbol{\Lambda}$ module $M$, and we define a pairing $\langle$,$\rangle between R$ and $S^{\text {ord }}(\psi)=S^{\text {ord }}(\psi) \otimes_{\Lambda} \check{\|}$ as follows:

$$
\langle h \otimes i, f \otimes \phi\rangle=\langle h \phi(i), f\rangle=\langle h, f \mid \phi(i)\rangle,
$$

where the pairing at the right-hand side is the pairing of Section 2.6 in Chapter 2 . We then define

$$
\ell_{\lambda}: S^{\text {ord }}(\psi) \rightarrow 0 \quad \text { by } \quad \ell_{\lambda}(f)=\left\langle H 1_{\lambda}, f\right\rangle .
$$

For a point $P \in \mathscr{X}_{\mathbb{1}}(\Omega)$, we have an $\mathrm{O}$-algebra homomorphism $\lambda_{P}=P \circ \lambda: R \rightarrow \overline{\mathbb{Q}}_{p}$. Suppose $P$ is positive and arithmetic. For a suitable $S_{F}$-tuple $\alpha=(\alpha(\mathfrak{P}))_{\mathfrak{\beta} \in S_{F}}$, we find $f(P) \in S_{\kappa(P)}^{\text {ord }}\left(N \mathfrak{P}^{\alpha} ; \overline{\mathbb{Q}}\right)$ such that $\mathbf{a}_{p}(y, f(P))=\lambda_{P}(\mathbb{T}(y))$ for $y \in \widehat{\mathfrak{r}} \cap F_{\mathbb{A}^{(\infty)}}^{\times}$. As seen in [41] Lemma 9.3, We have

$$
\ell_{\lambda}(h)=H(P) \frac{\left(f(P)^{c}\left|\tau\left(n \varpi^{\alpha}\right), h\right| e\right)_{\alpha}}{\left(f(P)^{c} \mid \tau\left(n \varpi^{\alpha}\right), f(P)\right)_{\alpha}}
$$

for all $h \in S_{\kappa(P)}\left(N \mathfrak{P}^{\alpha} ; \overline{\mathbb{Q}}\right)$, where $e$ is the $p$-ordinary projector, and $(,)_{\alpha}=(,)_{N \Re^{\alpha}}$ is the Petersson inner product on $S_{\kappa(P)}\left(N \mathfrak{B}^{\alpha} ; \mathbb{C}\right)$. Note that the expression on the left-hand side does not depend on the choice of $\alpha=(\alpha(\mathfrak{P}))_{\mathfrak{B}}$.

Now we take an $\boldsymbol{\Lambda}$-free submodule $\mathbb{X}$ in $\mathbb{K}$ containing $\mathbb{\mathbb { V }}$. Then $\mathbb{X} / \mathbb{\mathbb { }}$ is a torsion $\Lambda$-module. We recall our usage of symbol: $M^{*}$ for the $\mathcal{O}$-dual module of $M$ while $\check{M}$ indicates the $\boldsymbol{\Lambda}$-dual module of $M$. Define a subspace of $\mathscr{C}(\bar{Z} ; \mathcal{O})$ by

$$
\mathscr{C}(\bar{Z}(N) ; \mathcal{O})[\psi]=\{\phi \in \mathscr{C}(\bar{Z} ; \mathcal{O}) \mid \phi(\zeta x)=\psi(\zeta) \phi(x) \quad \text { for all } \quad \zeta \in \Delta(N)\} \text {. }
$$

We know, by restricting functions on $\bar{Z}(L)$ to $\mathbb{W}, \mathscr{C}(\bar{Z} ; \mathcal{O})[\psi] \cong \mathscr{C}(\mathbb{W} ; \mathcal{O})$ as $\boldsymbol{\Lambda}$ modules. Similarly, we define

$$
S(L ; \psi)=\left\{f \in S\left(L p^{\infty} ; 0\right)|f|\langle\zeta\rangle=\psi(\zeta) f \quad \text { for all } \quad \zeta \in \Delta(L)\right\}
$$

Then in exactly the same manner as in [41] p.381, we can show that

$$
M^{*} \widehat{\otimes}_{\mathbb{O}}(\mathscr{C}(\bar{Z} ; \mathcal{O})[\psi]) \otimes_{\Lambda} \check{\mathbb{X}} \cong M^{*} \widehat{\otimes}_{\mathbb{O}} \mathbb{X}^{*}
$$

and define, for the twisted trace operator $T_{L / N}$ in [41] p.367,

$$
\begin{aligned}
\Psi: M^{*} \widehat{\otimes}_{\mathcal{O}}(\mathscr{C}(\bar{Z} ; \mathcal{O})[\psi]) \stackrel{E * \varphi}{\longrightarrow} S(L ; \psi) \stackrel{T_{L / N}}{\longrightarrow} S(N ; \psi) \stackrel{e}{\rightarrow} S(\psi) \quad \text { and } \\
E *_{\lambda} \varphi: M^{*} \widehat{\otimes}_{\mathcal{O}} \mathbb{X}^{*} \longrightarrow \mathcal{O} \text { by } \quad \ell_{\lambda} \circ(\Psi \otimes \text { Id }) .
\end{aligned}
$$

The measure $E *_{\lambda} \varphi$ is independent of the auxiliary choice of $\mathbb{X}$, and as seen in [41] Lemma 9.4, we know $E *_{\lambda} \varphi \in M \widehat{\otimes}_{\mathbb{O}} \square$. 


\subsection{Proof of Theorem 6.5.1}

Now the proof of Theorem 6.5.1 goes in exactly the same way as that of Theorem 5.1 in [41] Section 10. We therefore briefly recall the argument and give the proof of the residue formula in details. We take a primitive homomorphism $\mu: h^{\text {ord }}\left(J p^{\infty} ; \mathcal{O}\right) \rightarrow \llbracket$ factoring through $h^{\text {ord }}(\chi)$. By the duality in Section 2.6 of Chapter 2 , we have $\mu^{*}: \rrbracket^{*} \rightarrow S^{\text {ord }}\left(J p^{\infty} ; \mathcal{O}\right)$. Set $L=N \cap J$. We take $\rrbracket^{*}$ as $M^{*}$ in Section 6.8 and $[L / J] \circ \mu^{*}$ as $\varphi$ in the previous section for the operator $[L / J]$ given in [41] Section 7B. Then $E *_{\lambda} \varphi$ gives an element of $\left(\nabla^{*} \widehat{\otimes}_{0} \rrbracket^{*}\right)^{*} \cong \llbracket \widehat{\otimes}_{0} \rrbracket$. We define an element $D^{\text {ord }}$ in the quotient field of $\square \widehat{\otimes}_{\mathcal{O}} \square$ by $\frac{E *_{\lambda} \varphi}{1 \otimes H}$. We can evaluate $D^{\text {ord }}$ at $(P, Q) \in \mathscr{A}_{\mathbb{1}} \times \mathscr{A}_{\mathbb{1}}$ satisfying (Ad) in exactly the same manner as in [41] Section 10 and get the evaluation formula as in Theorem 6.5.1. We now briefly recall the process in order to prove the residue formula.

We can choose $H$ so that $H(P) \neq 0$ and $H$ kills the congruence module $C_{0}(\lambda ; \square)$. We first compute $\Psi=T_{L / N} \circ e \circ(E * \varphi)$. By extending scalars if necessary, we

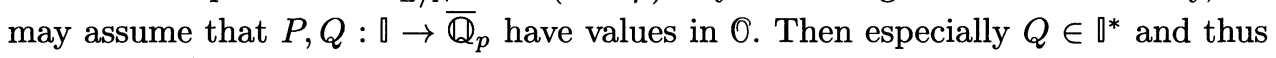
$Q \otimes \eta \in M^{*} \widehat{\otimes}_{\mathcal{O}} \mathscr{C}(\bar{Z} ; \mathcal{O})$ for any character $\eta$ of $\bar{Z}(L)$ with values in $\mathcal{O}$. We first compute $\Psi\left(Q \otimes \psi_{P}\right) \in S^{\text {ord }}(\psi)$, for the character $\psi_{P}: \bar{Z}(L) \rightarrow \mathcal{O}^{\times}$given by the restriction of $\lambda_{P}$ to $\bar{Z}(L)$, that is, $\psi_{P}=\psi \omega^{-[m(P)]} \mathcal{N}^{[m(P)]}$. Let $g(Q) \in S_{\kappa(Q)}^{\text {ord }}\left(J \mathfrak{B}^{\alpha} ; \mathcal{O}\right)$ be the normalized cusp form with Hecke eigenvalues $\mu_{Q}$. We write $\chi_{Q}$ for the restriction of $\mu_{Q}$ to $\bar{Z}(L)$. Then, regarding $Q \otimes \psi_{P} \in M^{*} \widehat{\otimes}_{\mathcal{O}}(\mathscr{C}(\bar{Z} ; \mathcal{O})[\psi])$, we have by definition

$$
\Psi\left(Q \otimes \psi_{P}\right)=T_{L / N} \circ e\left(g(Q) \mid[L / J] \cdot\left(E\left(\chi_{Q}^{-1} \psi_{P}\right) \mid\left(1-\delta\langle\mathfrak{d}\rangle \mathcal{N}(\mathfrak{d})^{2}\right)\right)\right.
$$

where $\delta=1$ or 0 according as $\chi=\psi$ or not. This can be rewritten as

$$
\left(\left(1-\delta\langle\mathfrak{x}\rangle \otimes\langle\mathfrak{x}\rangle^{-1}\right) \Psi \otimes \mathrm{Id}\right)(Q \otimes P)=T_{L / N} \circ e\left\{g(Q) \mid[L / J] \cdot E_{\mathfrak{x}, L}\left(\chi_{Q}^{-1} \psi_{P}\right)\right\}
$$

which implies

$$
\left(\left(1-\delta\langle\mathfrak{x}\rangle \otimes\langle\mathfrak{x}\rangle^{-1}\right) D^{o r d}\right)(P, Q)=\ell_{\lambda}\left(T_{L / N} \circ e\left\{g(Q) \mid[L / J] \cdot E_{\mathfrak{x}, L}\left(\chi_{Q}^{-1} \psi_{P}\right)\right\}\right) .
$$

This is true for densely populated points $(P, Q)$ in $\mathscr{X}_{\mathbb{1}} \times \mathscr{X}_{\mathbb{1}}$ and hence is true everywhere over $\mathscr{X}_{\mathbb{1}} \times \mathscr{X}_{\mathbb{1}}$. When $P=Q$, we still have

$$
\left(\left(1-\delta\langle\mathfrak{x}\rangle \otimes\langle\mathfrak{x}\rangle^{-1}\right) D^{o r d}\right)(P, P)=\ell_{\lambda}\left(T_{L / N} \circ e\left\{(g(P) \mid[L / J]) \cdot\left(E_{\mathfrak{x}, L}(\mathrm{id})\right)\right\}\right) .
$$

It is clear from the definition that $\ell_{\lambda}\left(T_{L / N} \circ e(g(P) \mid[L / J])\right)=\delta_{\lambda, \mu}$, where $\delta_{\lambda, \mu}$ is 1 or 0 according as $\lambda=\mu$ or not. Then the residue formula follows from (Res1) in Section 6.8. 


\section{7. $p$-Adic Rankin products in partially $\mathbf{C M}$ case}

We call $\lambda: \mathbf{h}=h^{\text {n.ord }}\left(N p^{\infty} ; \mathcal{O}\right) \rightarrow \square$ "of $C M$ type" if there exists a totally imaginary quadratic extension $L$ of the totally real field $F$ such that the isomorphism class $\pi(\lambda)$ contains the induced representation $\Phi$ of a character $\varphi: \mathscr{G}_{L} \rightarrow \rrbracket^{\times}$. In this case, using $p$-adic Hecke $L$-functions of Katz [52], we can construct a "genuine" $p$-adic Rankin product $L_{S}(\Phi \otimes \check{\rho})$ for $S=(\emptyset, I, \emptyset)$ and $\rho \in \pi(\mu)$ in Conjecture 5.5.1 of Chapter 5 and Conjectures 6.3 .1 and 6.3 .3 of Chapter 6.

\subsection{CM components of Hecke algebras}

Let $L$ be as above and write $C l_{L}\left(C p^{\alpha}\right)$ for the ray class group modulo $C p^{\alpha}$, where $C$ is an ideal of $L$ prime to $p$. Then we consider $C l_{L}\left(C p^{\infty}\right)=\lim _{\alpha} C l_{L}\left(C p^{\alpha}\right)$. By class field theory, we may identify $C l_{L}\left(C p^{\infty}\right)$ with the Galois group of the maximal ray class field modulo $C p^{\infty}$. Let $\mathscr{G}=\mathscr{G}_{F}$ and $\mathscr{H}=\mathscr{G}_{L}$. Let $\lambda: h^{n . o r d}\left(N p^{\infty} ; \mathbb{O}\right) \rightarrow \mathbb{0}$ be primitive and of CM type, and suppose that $\llbracket$ is torsion-free over $\Lambda$. Thus $\Phi=\operatorname{Ind}_{\mathscr{H}}^{\mathscr{G}} \varphi \in \pi(\lambda)$ for a character $\varphi: \mathscr{H} \rightarrow{\mathbb{q ^ { \times }}}^{\times}$. Since the ramification of $\pi(\lambda)$ outside $p$ is known to be finite, $\varphi$ factors through $C l_{L}\left(C p^{\infty}\right)$ for some $C$. We take the largest $C$ which we call the conductor of $\varphi$. Since $\pi(\lambda)$ is known to be irreducible, $\varphi\left(c \sigma c^{-1}\right) \neq \varphi(\sigma)$ for some $\sigma \in \mathscr{G}$, where $c$ is an element in $\mathscr{G}$ inducing a non-trivial automorphism on $L / F$. By (Gal) (iv) in Section 2.8 of Chapter 2, for $\mathfrak{B} \in S_{F}$, $\left.\Phi\right|_{D_{\mathfrak{\beta}}}\left(D_{\mathfrak{R}}=\mathscr{G}_{F_{\mathfrak{\beta}}}\right)$ is reducible, and one of its characters is given by the character $\delta_{\mathfrak{\beta}}: F_{\mathfrak{\beta}} \times \rightarrow \operatorname{Im}(\lambda)^{\times}$taking $y \in{F_{\mathfrak{\beta}}}^{\times}$to $\lambda(\mathbb{T}(y))$. There are two possibilities:

$$
D_{\mathfrak{B}} \subset \mathscr{H} \text { or }
$$

(ii) we can find $c$ as above in $D_{\mathfrak{\beta}}$ and $\varphi_{c}(\sigma)=\varphi\left(c \sigma c^{-1}\right)=\varphi(\sigma)$ for all $\sigma \in D_{\mathfrak{\beta}}$.

If Case (ii) occurs, $\varphi_{\mathfrak{\beta}}=\left.\varphi\right|_{D_{\mathfrak{\beta}} \cap \mathscr{H}}=\left.\phi_{\mathfrak{\beta}}\right|_{D_{\mathfrak{\beta}} \cap \mathscr{H}}$ for a character $\phi_{\mathfrak{R}}$ of $D_{\mathfrak{\beta}}$. Then the semi-simplification of $\left.\pi(\lambda)\right|_{D_{\mathfrak{\beta}}}$ is given by $\phi_{\mathfrak{\beta}} \oplus \phi_{\mathfrak{R}} \chi$ for the quadratic character $\chi$ corresponding to $L / F$. Thus we may assume that $\phi_{\mathfrak{B}}=\delta_{\mathfrak{B}}$ and $\eta=\operatorname{det}(\Phi)=\chi \delta_{\mathfrak{R}}^{2}$ on ${F_{\Re}}^{\times}$. We see that $\langle a\rangle=\eta(a)=\mathbb{T}(a)^{2}$ for $(a, a) \in \mathbf{G}$ sufficiently close to 1 , where $\mathbf{G}$ is the weight group as in Section 2.1 of Chapter 2. Thus Case (ii) never occurs. This implies $D_{\mathfrak{B}} \subset \mathscr{H}$; in other words, all prime factors in $S_{F}$ splits in $L$. We define 
a set $S$ of primes $\mathbf{P}$ of $L$ over $p$ such that $\left.\varphi\right|_{D_{\mathbf{P} c}}=\delta_{\mathfrak{R}}\left(D_{\mathbf{P}}=\varphi_{L_{\mathbf{P}}}\right)$. Then $S \cap S^{c}=\emptyset$ and $S \bigsqcup S^{c}$ is the set $S_{L}$ of all primes of $L$ over $p$. The subset $S$ can be regarded as a (p-adic $L)$ type $\left\{V_{\mathfrak{\beta}}^{-} \subset V(\Phi)\right\}$ as follows: for $\mathfrak{B} \in S_{F}$, writing $\mathbf{P}$ for the unique prime in $S$ above $\mathfrak{P}$, we take as $V_{\mathfrak{\beta}}^{-}=V_{\mathfrak{B}}^{-}(\lambda)$ the subspace of $V(\lambda)$ on which $D_{\mathbf{P}}$ acts via $\left.\varphi_{c}\right|_{D_{\mathbf{P}}}$. Thus the choice of $S$ gives rise to the choice of $\left\{V_{\Re}^{-}\right\}_{\Re \in S_{F}}$. Let $I_{L}$ be the set of all embeddings of $L$ into $\overline{\mathbb{Q}}$, and write $\Sigma$ for the set of embeddings made of $\sigma \in I_{L}$ such that $i_{p} \sigma$ induces a $p$-adic place in $S$. Then $I_{L}=\Sigma \bigsqcup \Sigma c$, that is, $\Sigma$ is a CM type. We call $S$ the $p$-adic CM type associated with $\Sigma$ and also call $\lambda$ of type $(L, S, \Sigma)$. Then as seen in [47] Section 6 and [48] Section 4, we have $N=N_{L / F}(C) D_{L / F}$ for the relative discriminant $D_{L / F}$ of $L / F$ and

$$
\lambda(\mathbb{T}(\mathfrak{n}))=\sum_{\mathfrak{a} \mathfrak{a}^{c}=\mathfrak{n}} \varphi([\mathfrak{a}]) \quad \text { if } \mathfrak{n} \text { is prime to } p,
$$

where $\mathfrak{a}$ runs over integral ideals of $L$ with norm $\mathfrak{n}$ and $[\mathfrak{a}]$ is the class of an ideal $\mathfrak{a}$ in $C l_{L}\left(C p^{\infty}\right)$. Here we understand $\varphi([a])=0$ if $\mathfrak{a}$ is not prime to $C p$. As for the value of $\lambda(\mathbb{T}(y))$ for $y \in F_{\mathfrak{\beta}}{ }^{\times}$, we get from the above argument

$(\mathrm{CM} 2) \lambda(\mathbb{T}(y))=\varphi(y)$ identifying $F_{\mathfrak{\beta}}$ with $L_{\mathbf{P} c}$ for $\mathbf{P} \in S$ with $\mathbf{P} \cap F=\mathfrak{B}$.

Moreover the character $\varphi$ satisfies the following properties:

(CM3) $\varphi$ is unramified at $S^{c}$ if $\lambda$ is p-ordinary;

(CM4) $\varphi_{P}$ is the $p$-adic avatar of a complex Hecke character $\varphi_{P}$ for $P \in \mathscr{A}_{0}$.

For any complex Hecke character $\omega: L^{\times} \backslash L_{\mathbb{A}}^{\times} \rightarrow \mathbb{C}^{\times}$of type $A_{0}$, we define its infinity type $\infty(\omega)=m \in \mathbb{Z}\left[I_{L}\right]$ by $\omega(x)=x^{m}=\prod_{\sigma} x^{\sigma m_{\sigma}}$ for $x \in L_{\infty}^{\times}$. Writing $m=\infty\left(\varphi_{P}\right)$ and $m(\Sigma)=\sum_{\sigma \in \Sigma} m_{\sigma} \sigma$, we have $-m-m c=m(P)+n(P)$, and by (CM2), we see that

$$
n(P)=-\left.\sum_{\sigma \in \Sigma c} m_{\sigma} \sigma\right|_{F}=-\left.m(\Sigma c)\right|_{F} \quad \text { and } \quad m(P)=-\left.m(\Sigma)\right|_{F} .
$$

\subsection{Periods of $M\left(\lambda_{P}\right)$ of CM type}

Let $M=M\left(\varphi_{P}\right)_{/ L}$ be the rank $1 \mathrm{CM}$ motive ([4]) attached to the Galois character $\varphi_{P}$ for a positive arithmetic point $P$. The field $E$ of coefficients of $M\left(\varphi_{P}\right)$ is given by $\mathbb{Q}\left(\varphi_{P}\right)$, which is generated over $\mathbb{Q}$ by the value of $\varphi_{P}$ on finite ideles $x$ away from $p$ (that is, $\left.x_{p}=1\right)$. Since $M\left(\varphi_{P}\right)$ is of rank $1, \mathscr{F}\left(M\left(\varphi_{P}\right)\right) \otimes_{L \otimes E, \sigma \otimes i_{\infty}} \mathbb{C} \neq 0 \Longleftrightarrow$ $\sigma \in \Sigma c$. Since $\mathscr{F}\left(M\left(\varphi_{P}\right)\right) \otimes_{L \otimes E, \sigma \otimes i} X=H_{D R}(M) \otimes_{L \otimes E, \sigma \otimes i} X(\sigma \in \Sigma c)$ for $X=\mathbb{C}$ and $B_{H T}$, we see (e.g. [42] Lemma 1.2) that

$$
c_{*, \sigma}^{ \pm}\left(\Phi_{P}\right)=\delta_{*, \sigma}\left(\varphi_{P}\right) \neq 0 \quad \text { and } \quad \alpha_{ \pm}(\Phi) \in \mathbb{Q}^{\times} .
$$


Thus for any $i_{\infty}, L_{T, i_{\infty}}(\Phi)=L_{S, i_{\infty}}(\varphi)$ (for $\left.T=(\emptyset, I, \emptyset)\right), L_{T, i_{\infty}}(\Phi \otimes \check{\rho}$ ) and $L_{p, i_{\infty}}(A d(\Phi))$ are automatically normalized (see (NP) in Chapter 4 and Theorems 5.2.1 and 5.2.2 in Chapter 5); so, we drop $i_{\infty}$ from the notation (for example, we write $L_{T}(\Phi)$ for $\left.L_{T, i_{\infty}}(\Phi)\right)$. For $X=B_{H T}$, there could be two comparison isomorphisms $I_{F a}$ and $I_{R T}$. The isomorphism $I_{F a}$ is due to Faltings [21] which we used to construct $c_{H T, \iota}^{ \pm}(M)$ in Section 3.2 of Chapter 3, and the other $I_{R T}$ is due to Raynaud-Tate for abelian varieties [68]. Let $\mathscr{C} \mathcal{M}$ be the category of absolute Hodge motives defined over $\overline{\mathbb{Q}}$ generated by Artin motives and abelian varieties of CM-type. Thus $\mathscr{C} \mathcal{M}$ is generated by $H^{1}(A)$ for abelian varieties $A$ as above and Artin motives. If a functorial extension to $\mathscr{G} \mathcal{M}$ of $I_{R T}$ originally defined for $H^{1}$ of $A$ exists, then it is unique. We assume the existence of the extension:

$\left(\mathrm{C}_{R T}\right)$ There exists a natural transformation between the following fibre functors on GM:

$$
I_{R T}: H_{p}(M) \otimes_{\mathbb{Q}_{p}} B_{H T} \cong H_{D R}(M) \otimes_{\overline{\mathbb{Q}}, i_{p} \sigma} B_{H T}
$$

By [21] and [2] Theorem 0.3, $I_{F a}$ induces another natural transformation on $\mathscr{C} \mathcal{M}$ :

$$
I_{F a}: H_{p}(M) \otimes_{\mathbb{Q}_{p}} B_{H T} \cong H_{D R}(M) \otimes_{\overline{\mathbb{Q}}, i_{p} \sigma} B_{H T}
$$

Thus $\sigma_{e t}: I_{F a}^{-1} \circ I_{R T}$ (resp. $\sigma_{D R}=I_{F a} \circ I_{R T}^{-1}$ ) gives an element of the motivic Galois group $\mu_{e t}\left(B_{H T}\right)$ (resp. $\mathcal{M}_{D R}\left(B_{H T}\right)$ ) for $\mu_{e t}=A u t_{\mathscr{C}} \mathcal{M}\left(H_{p}\right)$ (resp. $M_{D R}=$ $\left.\operatorname{Aut}_{\mathscr{C} M}\left(H_{D R}\right)\right)$. Since $\sigma_{D R}$ commutes with the Galois action of $\mathscr{G}_{\mathbb{Q}_{p}}, \sigma_{D R} \in$ $M_{D R}\left(\mathbb{Q}_{p}\right)$. We can use $I_{R T}$ and $I_{F a}$ to define the period $c_{p, i_{\infty}}^{ \pm}(M)$, which we write $c_{p}^{ \pm}\left(M ; I_{X}\right)$, if necessary, to indicates its dependence on $I_{X}$ for $X=F a$ and $R T$. Since $I_{F a}=\sigma_{D R} I_{R T}, c_{p}^{ \pm}\left(M ; I_{R T}\right) \equiv c_{p}^{ \pm}(M) \bmod \mathbb{Q}_{p}^{\times}$.

On the other hand, by using the monomial relation of CM periods due to Shimura, Deligne and Blasius [4] for complex periods and to Gillard [27] and Blasius [2] for $p$-adic periods, we can write $c_{\infty}^{ \pm}\left(\Phi_{P}\right)$ and $c_{p}^{ \pm}\left(\Phi_{P} ; I_{X}\right)$ up to algebraic numbers in terms of the periods of an abelian variety with complex multiplication of type $(L, \Sigma)$ : We briefly recall the definition of the period from [52] and [47]. For that, we need some more notation. We write $\mathcal{V}=\mathcal{O}_{\Omega}$ for the $p$-adic integer ring of $\Omega$. We then put $\mathscr{V}_{0}=i_{p}^{-1}(\mathscr{V})$. Let $\mathscr{R}$ be the integer ring of $L$. We fix an element $0 \neq \delta \in \mathscr{R}$ such that

$$
\delta^{c}=-\delta \text { and } \operatorname{Im}\left(\delta^{\sigma}\right)>0 \text { for all } \sigma \in \Sigma \text {; }
$$

$(\Delta 2)$ The alternating form $\langle u, v\rangle=\left(u^{c} v-v^{c} u\right) / 2 \delta$ induces an isomorphism $\mathscr{R} \bigwedge_{\mathfrak{r}} \mathscr{R} \cong \mathfrak{D}^{-1} \mathfrak{c}$ for an ideal $\mathfrak{c}$ prime to $p$,

where $\mathfrak{D}$ is the different of $L / \mathbb{Q}$. We can algebraize the complex torus $\mathbb{C}^{\Sigma} / \mathscr{R}^{\Sigma}$ for

$$
\mathscr{R}^{\Sigma}=\left\{\left(a^{\sigma}\right)_{\sigma \in \Sigma} \in \mathbb{C}^{\Sigma} \mid a \in \mathscr{R}\right\}
$$


and find an abelian scheme $A=A_{\Sigma}$ over $\mathscr{V}_{0}$ with $\mathfrak{c}$-polarization $\mathscr{P}$ induced by $\langle$,$\rangle . Let u=\left(u_{\sigma}\right)_{\sigma \in \Sigma}$ be the coordinate of $\mathbb{C}^{\Sigma}$. We put $\omega_{\infty}=d u=\sum_{\sigma \in \Sigma} d u_{\sigma}$ which is a generator of $\Omega_{A / \mathbb{C}}$ over the ring $\mathbb{C}^{\Sigma}$. Identifying the $S$-adic completion $\mathscr{R}_{S}$ of $\mathscr{R}$ with $\mathfrak{r}_{p}$, we have a trivialization $i: F_{p} / \mathfrak{D}_{p}^{-1} \rightarrow A(\mathbb{C})$. This extends to a trivialization $i: \mu_{p^{\infty}} \otimes \mathfrak{D}^{-1} \subset A$ over $\mathscr{V}_{0}$. Then $i$ induces an isomorphism of formal groups $i: \widehat{\mathbf{G}}_{m} \otimes_{\mathbb{Z}} \mathfrak{D}^{-1} \cong \widehat{A}$ for the formal completion $\widehat{A}$ of $A$ along the origin. Writing $\mathbf{G}_{m}=\operatorname{Spec}\left(\mathbb{Z}\left[t, t^{-1}\right]\right), \mathbf{G}_{m}$ has a nowhere vanishing differential $\frac{d t}{t}$. Then we put $\omega_{p}=i *\left(\frac{d t}{t}\right)$ on $A$, which is a generator of $\Omega_{A / \mathcal{V}}$ over the ring $\mathscr{V} \otimes_{\mathbb{Z}} \mathscr{R}_{S}$. We pick a global section $\omega$ of $\Omega_{A / V_{0}}$ which is a generator over the ring $\overline{\mathbb{Q}} \otimes_{\mathbb{Z}} \mathscr{R}$. We can supplement $\omega$ with a section $\eta \in H^{1}\left(A, O_{A}\right)$ for the structure sheaf $O_{A}$ of $A_{/ V_{0}}$ so that $H_{D R}(A)$ is generated by $(\omega, \eta)$ over the ring $V_{0} \otimes_{\mathbb{Z}} L$. We also choose a generator $\alpha$ so that $H_{1}(A ; \mathbb{Q})=L \alpha$ and $\int_{\alpha} \omega_{\infty}=1$. Then the two periods $\Omega_{p}=\Omega_{p}(R T ; \Sigma)$ in $\left(\mathscr{V} \otimes_{\mathbb{Z}} \mathscr{R}_{S}\right)^{\times} \subset\left(\mathscr{V}^{\times}\right)^{\Sigma}$ and $\Omega_{\infty}=\Omega_{\infty}(\Sigma)$ in $\left(\mathbb{C}^{\times}\right)^{\Sigma}$ are defined by

$$
\omega=\Omega_{p} \omega_{p} \quad \text { and } \quad \omega=\Omega_{\infty} \omega_{\infty}\left(\Leftrightarrow \Omega_{\infty}=\int_{\alpha} \omega\right) .
$$

Then we have

$$
c_{\infty, \sigma}^{ \pm}\left(\pi\left(\lambda_{P}\right)\right) \equiv(2 \pi i)^{n(P)} \Omega_{\infty}^{m(P)-n(P)} \bmod \overline{\mathbb{Q}}^{\times},
$$

and we expect to have

$$
c_{p, \sigma}^{ \pm}\left(\pi\left(\lambda_{P}\right) ; I_{F a}\right) \equiv c_{p, \sigma}^{ \pm}\left(\pi\left(\lambda_{P}\right) ; I_{R T}\right) \equiv \Omega_{p}^{m(P)-n(P)} \quad \bmod \overline{\mathbb{Q}}^{\times}
$$

where $\Omega_{*}^{d}=\prod_{\sigma \in \Sigma} \Omega_{*, \sigma}^{d_{\sigma}}$ for $d \in \mathbb{Z}[I]$.

We now look at the specific abelian scheme $A_{\Psi / \mathcal{V}_{0}}$ of CM-type $(L, \Psi)$. We choose for each $\Psi$, generators $b_{\Psi}$ and $\eta_{\Psi}$ so that $H_{e t}^{1}\left(A_{\Psi}, \mathbb{Q}_{p}\right)=L \otimes_{\mathbb{Z}_{p}} \mathbb{Q}_{p} b_{\Psi}$ and $H_{D R}^{1}\left(A_{\Psi}\right)=L \otimes_{\mathbb{Q}} \overline{\mathbb{Q}} \eta_{\Psi}$ regarding $A_{\Psi}$ is defined over $\overline{\mathbb{Q}}$. Then we could have defined $\Omega_{p}(R T ; \Psi)$ by $I_{R T}\left(b_{\Psi}\right)=\Omega_{p}(R T ; \Psi) \eta_{\Psi}$, because the Tate module $T_{p}(A) \otimes_{\mathbb{Z}} \mathbb{Q}$ is the dual of $H_{e t}^{1}\left(A_{\Psi}, \mathbb{Q}_{p}\right)$. We define $\Omega_{p}(F a ; \Psi)$ by $I_{F a}\left(b_{\Psi}\right)=\Omega_{p}(F a ; \Psi) \eta_{\Psi}$. Note that $\Omega_{p}(F a ; \Psi)=c_{\Psi}(\sigma) \Omega_{p}(R T ; \Psi)$, where $c_{\Psi}(\sigma)$ is an element of $L \otimes_{\mathbb{Z}_{p}} \mathbb{Q}_{p}$ induced by $\sigma_{D R}$. Since we can always realize $M_{P}$ inside $\otimes_{\Psi} H^{1}\left(A_{\Psi}\right)^{\otimes k_{\Psi}}$ for a suitable choice of integers $k_{\Psi}$, we can write down up to algebraic numbers $c_{p}^{ \pm}\left(M ; I_{X}\right)$ as a monomial of periods $\Omega_{p}(X ; \Psi)$. The form of expression is independent of $X=R T$ or $F a$ under $\left(\mathrm{C}_{R T}\right)$, and the ratio of two periods are given by the same monomial of $c_{\Psi}(\sigma)$. In particular, if $\otimes_{\Psi} H^{1}\left(A_{\Psi}\right)^{\otimes k_{\Psi}} \cong \otimes_{\Psi} H^{1}\left(A_{\Psi}\right)^{\otimes k_{\Psi}^{\prime}}$, then $\frac{\prod_{\Psi} \Omega_{p}(X ; \Psi)^{k_{\Psi}}}{\prod_{\Psi} \Omega_{p}(X ; \Psi)^{k_{\Psi}^{\prime}}}$ is independent of $X$. Thus

$$
\begin{aligned}
& \left(\operatorname{Pd}_{R T F}\right) \\
& \quad c_{p}^{ \pm}\left(M_{P}(1) ; I_{R T}\right) / \Omega_{p}(R T ; \Sigma)^{m(P)-n(P)}=c_{p}^{ \pm}\left(M_{P}(1) ; I_{F a}\right) / \Omega_{p}(F a ; \Sigma)^{m(P)-n(P)} .
\end{aligned}
$$


We simply write $\Omega_{p}(P)=\Omega_{p}(R T ; P)\left(\right.$ resp. $\left.\Omega_{\infty}(P)\right)$ for $\Omega_{p}(R T ; \Sigma)^{m(P)-n(P)}$ (resp. $\left.(2 \pi i)^{n(P)} \Omega_{\infty}^{m(P)-n(P)}\right)$. Since

$$
i_{p}^{-1}\left(c_{p}^{ \pm}\left(M_{P}(1) ; I_{R T}\right) / \Omega_{p}(R T ; P)\right)=i_{\infty}^{-1}\left(c_{\infty}^{ \pm}\left(M_{P}(1)\right) / \Omega_{\infty}(P)\right)
$$

by $\left(\mathrm{C}_{R T}\right)$, we can replace the transcendental factor $\Omega_{p}(R T ; \Sigma)^{m(P)-n(P)}$ by $c_{p}^{ \pm}\left(M_{P}(1) ; I_{R T}\right)$. It is plausible that $I_{R T}$ coincides with $I_{F a}$ on $H^{1}$ of abelian varieties (and thus $I_{R T}=I_{F a}$ ), but it seems that this is not yet verified ([50] 4.3.5). Anyway we assume

(U) There exists an unit $\varepsilon \in \mathbb{Q}^{\times}$such that $\frac{c_{p}^{+}\left(M_{P}(1) ; I_{R T}\right)}{c_{p}^{+}\left(M_{P}(1) ; I_{F a}\right)}=\varepsilon(P)$ for all motivic $P$ of type $S$.

If $I_{F a}=I_{R T}$, then $\varepsilon=1$. For the character $\varphi: \mathscr{H} \rightarrow \mathbb{Q}^{\times}$, Katz (see [52] and [47] Theorem II) found a $p$-adic $L$-function $L_{S}(\varphi) \in \llbracket \widehat{\otimes}_{\mathcal{O}} \mathcal{V}$ satisfying the following interpolation property for all positive arithmetic points $P$

$\frac{L_{S}(P, \varphi)}{\Omega_{p}(P)}=\left(\mathscr{R}^{\times}: \mathfrak{r}^{\times}\right) W_{p}\left(\varphi_{P}\right)(-1)^{m(P)}|D|^{-1 / 2}(-2 \delta)^{n(P)} E(P) \frac{\Gamma_{F}(m(P)) L\left(0, \varphi_{P}^{-1}\right)}{\Omega_{\infty}(P)}$,

where $D$ is the discriminant of $F / \mathbb{Q}, \Gamma_{F}(d)=\prod_{\sigma \in I} \Gamma\left(d_{\sigma}\right)$ for the gamma function $\Gamma(s),(2 \delta)^{n}=\prod_{\sigma \in \Sigma}\left(2 \delta^{\sigma}\right)^{n_{\sigma}}, W_{p}\left(\varphi_{P}\right)$ is the Gauss sum factor defined in [47] Theorem II and

$$
E(P)=\prod_{\mathfrak{\Omega} \mid C}\left(1-\varphi_{P}^{-1}(\mathfrak{\Omega})\right) \prod_{\mathbf{P} \in S}\left\{\left(1-\varphi_{P}^{-1}\left(\mathbf{P}^{c}\right)\right)\left(1-\varphi_{P}(\mathbf{P}) N(\mathbf{P})^{-1}\right)\right\} .
$$

Here we have written $L_{S}(P, \varphi)=\int \varphi_{P}^{-1} d \mu$ for the Katz's measure given in [47] Theorem II. Note that the ratio $\frac{\Omega_{p}(P)}{\Omega_{\infty}(P)}$ is by definition independent of the choice of the global differential $\omega$, and hence the equality (7.2.3) makes sense. We now rewrite the value using the complex functional equation or its $p$-adic counter part in [47] Theorem II. The result is

$L_{S}(P, \varphi)=\left(\mathscr{R}^{\times}: \mathfrak{r}^{\times}\right) N(\mathfrak{c})^{-1} \varphi_{P}(\mathfrak{c}) W\left(\varphi_{P}\right)|D|^{-1 / 2}(2 \delta)^{n(P)} E(P) \frac{\Gamma_{F}(m(P)) L\left(1, \varphi_{P}\right)}{\Omega_{\infty}(P)}$ where $W\left(\varphi_{P}\right)=\varphi_{P, \infty}(-1) W^{\prime}\left(\varphi_{P}^{-1}\right) W_{p}\left(\left(\varphi_{P}\right)_{c}\right)$ for the Gauss sums $W_{p}$ and $W^{\prime}$ defined in [47] Theorem II. If $I_{R T}=I_{F a}$, as we have seen, $\Omega_{p}(P) / c_{p}^{+}\left(\varphi_{P}\right)$ and $\Omega_{\infty}(P) / c_{\infty}^{+}\left(\varphi_{P}\right)$ are both algebraic and equal in $\overline{\mathbb{Q}}$. Under the milder assumption (U), $L_{S}(\varphi)$ is a genuine $p$-adic $L$-function up to units if $p>2$. Assuming the complete splitting of $p$ in $F$, the extensibility $\left(\mathrm{C}_{R T}\right)$ is basically proven by Gillard [27]. The full splitting of $p$ in $F$ guarantees that any complex CM type is actually ordinary; 
thus, $A_{\Sigma}$ falls in the ordinary case treated by [27]. In this case, we could say that $L_{S}(\varphi)$ is "genuine" with respect to $I_{R T}$ replacing $I_{F a}$ by $I_{R T}$ in the definition of $c_{p}^{+}\left(\varphi_{P}\right)$.

\subsection{Genuine $p$-adic Rankin products}

We find, by computing Euler factors of $L_{i_{\infty}}\left(s, A d\left(\Phi_{P}\right)\right)$ for an arithmetic point $P$,

$$
L_{i_{\infty}}\left(1, A d\left(\Phi_{P}\right)\right)=L_{i_{\infty}}\left(1, \varphi_{P}^{-1}\left(\varphi_{P}\right)_{c}\right) L_{i_{\infty}}\left(1, \chi_{L}\right),
$$

where $\left(\varphi_{P}\right)_{c}(\sigma)=\varphi_{P}\left(c \sigma c^{-1}\right)$, and $\chi_{L}$ is the quadratic character corresponding to $L / F$. We suppose

(Prim) Every prime factor of $C$ outside the conductor of $\varphi^{-1} \varphi_{c}$ is either ramified or inert over $F$.

Under this condition, the imprimitive adjoint $L$-function obtained from $\lambda_{P}$ is just a non-zero constant multiple of the primitive one; thus, under (Prim), we need not worry about the vanishing of the extra Euler factors. We thus define

$$
L_{p}(A d(\Phi))=L_{S}\left(\varphi^{-1} \varphi_{c}\right) c_{p}^{+}\left(\chi_{L} \mathcal{N}^{-1}\right) \frac{L\left(1, \chi_{L}\right)}{c_{\infty}^{+}\left(\chi_{L} \mathcal{N}^{-1}\right)},
$$

where $L_{S}\left(\varphi^{-1} \varphi_{c}\right)$ is the Katz $p$-adic $L$-function of type $S$ studied in the previous section. In the CM case, the restriction of $\pi(\lambda)$ to $\mathscr{G}_{F_{\Re}}$ is reducible, and hence we have two choices of $V_{\mathfrak{B}}^{-}\left(\lambda_{P}\right)$. Our choice is given by $S$ as in Section 7.1 and gives a unique choice of $V_{\mathfrak{\beta}}^{-}\left(A d\left(\Phi_{P}\right)\right)$ as explained in Section 5.6 of Chapter 5. Let $\mu: h^{\text {n.ord }}\left(J p^{\infty} ; \mathcal{O}\right) \rightarrow \square$ be another primitive $\Lambda$-algebra homomorphism. We have a $p$-adic $L$-function $D_{p}(\pi(\lambda) \otimes \check{\pi}(\mu))$ in the field of fractions of $\square \widehat{\otimes}_{\mathbb{O}} \square$. Then we simply define for $T=(\emptyset, I, \emptyset)$

$$
L_{T}(\Phi \otimes \check{\pi}(\mu))=L_{p}(A d(\Phi)) D_{p}(\pi(\lambda) \otimes \check{\pi}(\mu)) .
$$

Here we could have written $L_{T}(\Phi \otimes \check{\rho})$ for $L_{T}(\Phi \otimes \check{\pi}(\mu))$ taking $\rho \in \pi(\mu)$, but it is actually independent of the choice of $\rho$ by Theorem 5.2.1 and (NP) in Section 4.3 of Chapter 4.

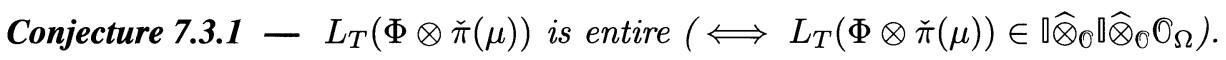

This conjecture implies Conjecture 6.3.3 in Chapter 6 in our situation if $I_{F a}=I_{R T}$. Conjecture 7.3.1 follows from the conjecture in [47] p.192 (see also Conjecture 5.6.1 in Chapter 5 in the text). This conjecture is known to be true by [55] and [71] (see also [48]) when $F=\mathbb{Q}$ and hence, Conjecture 7.3.1 hold in this case. We will show in the following paragraph that Conjecture 7.3.1 holds when $\mu$ is also of CM-type for general totally real $F$. 


\subsection{Totally CM Case}

Let $L^{\prime}$ be another CM quadratic extension of $F$. We assume that $\mu$ is of CM type with data $\left(L^{\prime}, S^{\prime}, \Sigma^{\prime}, \psi\right)$ like $(L, S, \Sigma, \varphi)$. Thus $\pi(\mu)$ has an induced representation $\Psi$ of a character $\psi: \mathscr{G}_{L^{\prime}} \rightarrow \rrbracket^{\times}$. We define the $2 \times 2$ Hecke matrix $A_{\mathfrak{l}}$ for $\lambda_{P}$ so that $\operatorname{det}\left(1-A_{\mathfrak{l}} X\right)=1-\lambda_{P}(\mathbb{T}(\mathfrak{l})) X+\lambda_{P}(\langle\mathfrak{l}\rangle) X^{2}$ for each prime $\mathfrak{l}$ outside $p$. Thus the above polynomial can be of degree less than 2 only when $\mathfrak{l}$ divides the conductor of $\lambda_{P}$. We define $B_{\mathfrak{l}}$ similarly for $\mu_{Q}$. Then we define imprimitive Euler products

$$
\begin{aligned}
\mathscr{L}\left(s, \lambda_{P} \otimes \check{\mu}_{Q}\right) & =\prod_{\mathfrak{l}} \operatorname{det}\left(1-A_{\mathfrak{l}} \otimes B_{\mathfrak{l}} N(\mathfrak{l})^{-s}\right)^{-1}, \\
\mathscr{L}\left(s, A d\left(\lambda_{P}\right)\right) & =\prod_{\mathfrak{l}} \operatorname{det}\left(1-A d\left(A_{\mathfrak{l}}\right) N(\mathfrak{l})^{-s}\right)^{-1} .
\end{aligned}
$$

Then the $p$-adic $L$-function $D_{p}=D_{p}(\pi(\lambda) \otimes \check{\pi}(\mu))$ basically interpolates the ratio: $\frac{\mathscr{L}\left(1, \lambda_{P} \otimes \check{\mu}_{Q}\right)}{\mathscr{L}\left(1, A d\left(\lambda_{P}\right)\right)}$. It is obvious that $\mathscr{L}\left(s, \lambda_{P} \otimes \check{\mu}_{Q}\right)$ (resp. $\left.\mathscr{L}\left(s, A d\left(\lambda_{P}\right)\right)\right)$ is equal to $L\left(s, \Phi_{P} \otimes \check{\Psi}_{Q}\right)$ (resp. $L\left(s, A d\left(\Phi_{P}\right)\right)$ up to finitely many Euler factors. By computing Euler factors of $L\left(s, \lambda_{P} \otimes \check{\mu}_{Q}\right)$ for two arithmetic points $P$ and $Q$, we find

$$
\mathscr{L}\left(s, \lambda_{P} \otimes \check{\mu}_{Q}\right)=E(s) \times \begin{cases}L\left(s, \varphi_{P} \psi_{Q}^{-1}\right) L\left(s, \varphi_{P}\left(\psi_{Q}\right)_{c}^{-1}\right) & \text { if } L^{\prime}=L, \\ L\left(s, \widehat{\varphi}_{P} \widehat{\psi}_{Q}^{-1}\right) & \text { if } L^{\prime} \neq L,\end{cases}
$$

where $E(s)$ is a finite Euler factor, and $\widehat{\varphi}_{P}$ (resp. $\widehat{\psi}_{Q}$ ) is the restriction of $\varphi_{P}$ (resp. $\left.\psi_{Q}\right)$ to $\mathscr{G}_{L L^{\prime}}$ for the composite $L L^{\prime}$. Let $E_{p}(s)$ be the Euler factor of $E(s)$ outside

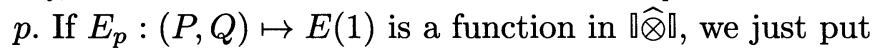

$$
L_{T}(\Phi \otimes \check{\Psi})=E_{p} \times \begin{cases}L_{S}\left(\varphi \psi^{-1}\right) L_{S}\left(\varphi \psi_{c}^{-1}\right) & \text { if } L^{\prime}=L \\ L_{S}\left(\widehat{\varphi} \widehat{\psi}^{-1}\right) & \text { if } L^{\prime} \neq L\end{cases}
$$

where the $L$-function appearing on right-hand side is the corresponding Katz $p$-adic $L$-functions of type $S$, and $\widehat{\varphi}$ (resp. $\widehat{\psi}$ ) is the restriction of $\varphi$ (resp. $\psi$ ) to $\mathscr{G}_{L L^{\prime}}$. Here the subset $S$ in $S_{L L^{\prime}}$ is made of $p$-adic places given by the extensions of places in $S$ to $L L^{\prime}$. Then the above $L_{T}=L_{T}(\Phi \otimes \check{\Psi})$ gives the desired "genuine" $p$-adic Rankin product. As was done in [47] p. 250-51 when $L=L^{\prime}$, one can check that the $p$-Euler factors of $D_{p}$ and $L_{T} / L_{p}$ for $L_{p}=L_{p}(A d(\Phi))$ match in general. Thus we only need to prove $E_{p} \in \mathbb{\otimes} \mathbb{\|}$ as follows. Let $\Xi$ be the set of prime ideals outside of $p$ satisfying the following condition:

$$
U_{\mathfrak{l}}=\left(V\left(\Phi_{P}\right) \otimes V\left(\check{\Psi}_{Q}\right)\right)^{I_{\mathfrak{l}}} /\left(V\left(\Phi_{P}\right)^{I_{\mathfrak{I}}}\right) \otimes\left(V\left(\check{\Psi}_{Q}\right)^{I_{\mathfrak{l}}}\right) \neq\{0\} .
$$

Then $E_{p}$ is the product of Euler factors at primes in $\Xi$. We can also check that $\Xi$ is independent of $P$, because the Euler factor depends only on the restriction of $\Phi$ and $\Psi$ to $I_{\mathfrak{l}}$ whose image is finite and hence independent of $P$ and $Q$. Since $E_{p}$ is just

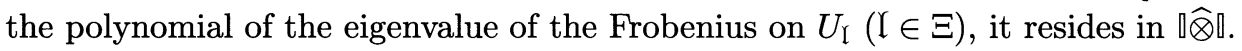





\section{8. $p$-Ordinary Katz $p$-adic $L$-functions}

Suppose that $\lambda: h^{\text {ord }}\left(N p^{\infty} ; \mathcal{O}\right) \rightarrow \square$ is of CM type, and $\Phi=\operatorname{Ind}_{\mathscr{H}}^{\mathscr{G}}(\varphi) \in \pi(\lambda)$ for a character $\varphi: \mathscr{H} \rightarrow \mathbb{1}^{\times}$. We sketch a proof of the fact that the ordinary Katz $p$-adic $L$-function $L_{p}(\Phi)=L_{S}(\varphi)$, under the Leopoldt conjecture for $F$ and $p$, satisfies (G) in Section 4.4 of Chapter 4.

\section{1 $p$-Ordinary Eisenstein measures}

We use the notation introduced in Chapter 7. Consider the maximal unramified extension $K_{0}$ in $\Omega$ over $\mathbb{Q}_{p}$. Let $\mathscr{V}$ be a finite extension of the $p$-adic integer ring of $K_{0}$. Let $\mathfrak{f}$ be an integral ideal and $\mathfrak{c}$ be a fractional ideal prime to $p \mathfrak{f}$. For each ideal $\mathfrak{a}$, we write $\mathfrak{a}^{*}=\mathfrak{a}^{-1} \mathfrak{b}^{-1}$. We write $V\left(\mathfrak{c}, \mathfrak{f}^{2} ; R\right)$ for the space of Katz's $p$-adic modular functions of prime-to-p-level $\mathfrak{f}$ with coefficients in $R$ (see [47] 1.5 for precise definition). It is defined geometrically as an affine $p$-adic ring whose formal spectrum is the solution of a $p$-adic ordinary formal moduli problem classifying test objects made of a Hilbert-Blumenthal abelian acheme $A$, its c-polarization $\mathscr{P}$ and $p^{\infty} \mathfrak{f}^{2}$-level structure $i$, everything being defined over a ring $R$. For any choice of fractional ideals $\mathfrak{a}, \mathfrak{b}$ with $\mathfrak{a b}^{-1}=\mathfrak{c}$, evaluation of such modular function at the Tate abelian variety $\left(\operatorname{Tate}_{\mathfrak{a}, \mathfrak{b}}(q), \mathscr{P}_{c a n}, i_{c a n}\right)$ (described in [52]) gives an isomorphism of $V\left(\mathfrak{c}, \mathfrak{f}^{2} ; R\right.$ ) onto the $p$-adic completion of the union (with respect to the level $\mathfrak{f}^{2} p^{\alpha}(\alpha=1,2, \ldots, \infty)$ and weight $k$ ) of classical holomorphic modular forms with $i_{p}$-integral coefficients.

Over $\mathbb{C}$, the category of the test objects is equivalent to the category of triples $(\mathscr{L}, \lambda, i)$, where $\mathscr{L}$ is an r-lattice in $F_{\mathbb{C}}=F \otimes_{\mathbb{Q}} \mathbb{C}, i$ is a level $p^{\infty} \mathfrak{f}^{2}$ structure (that is, an injective homomorphism $\left.i: F_{p} / \mathfrak{D}_{p}^{-1} \times\left(\mathfrak{f}^{2}\right)^{*} / \mathfrak{D}^{-1} \rightarrow p^{-\infty} \mathscr{L} / \mathscr{L} \times \mathfrak{f}^{-2} \mathscr{L} / \mathscr{L}\right)$, and $\lambda$ is an isomorphism $\mathscr{L} \bigwedge_{\mathfrak{r}} \mathscr{L} \cong \mathfrak{D}^{-1} \mathfrak{c}^{-1}$. The link between $A$ and $\mathscr{L}$ is given by $A(\mathbb{C}) \cong F_{\mathbb{C}} / \mathscr{L}$. The Eisenstein measure is a bounded measure with values in $V=V\left(\mathfrak{c}, \mathfrak{f}^{2} ; \mathscr{V}\right)$ supported on an open subset of $T=\left\{\mathfrak{r}_{p} \times(\mathfrak{r} / \mathfrak{f})\right\} \times\left\{\mathfrak{r}_{p} \times(\mathfrak{r} / \mathfrak{s})\right\}$ $(\mathfrak{g} \supset \mathfrak{f})$. We consider the space of locally constant functions

$$
\mathscr{C}_{k}^{ \pm}=\left\{\phi: T \rightarrow \mathbb{C} \mid \phi\left(\varepsilon^{ \pm 1} x, \varepsilon^{ \pm 1} x^{\prime}, \varepsilon y, \varepsilon y^{\prime}\right)=N(\varepsilon)^{k} \phi\left(x, x^{\prime}, y, y^{\prime}\right)\right\}
$$


Each $\phi \in \mathscr{C}_{k}^{-}$, we associate an Eisenstein series of weight $k$ by

(E) $\quad E_{k}((\mathscr{L}, \lambda, i) ; \phi, \mathfrak{c})=\left.\frac{(-1)^{k t} \Gamma_{F}(k t+s t)}{\sqrt{|D|}} \sum_{w \in \mathfrak{f}^{-1} p^{-\infty} \mathscr{L} / \mathfrak{r} \times} \frac{P \phi(w)}{N(w)^{k}|N(w)|^{2 s}}\right|_{s=0}$,

where $P$ is the partial Fourier transform with respect to $\left(x, x^{\prime}\right)$ which sends $\phi$ to a function on $\mathfrak{f}^{-1} p^{-\infty} \mathscr{L}$ supported by an $\mathfrak{r}$-lattice (see [47] (2.2)). Then we get, if $k \geq 2$,

$$
\begin{aligned}
& E_{k}(\phi, \mathfrak{c})\left(\operatorname{Tate}_{\mathfrak{a}, \mathfrak{b}}(q), \mathscr{P}_{c a n}, i_{c a n}\right)=N(\mathfrak{a})\left\{2^{-[F: \mathbb{Q}]} L(1-k ; \phi, \mathfrak{a})\right. \\
& \left.\quad+\sum_{0 \ll \xi \in \mathfrak{a} \mathfrak{b}(a, b) \in\{\mathfrak{a} \times \mathfrak{b}\} / \mathfrak{r} \times, a b=\xi} \phi(a, b) \operatorname{sgn}(N(a)) N(a)^{k-1} q^{\xi}\right\},
\end{aligned}
$$

where $L(s, \phi ; \mathfrak{a})=\sum_{\xi \in(\mathfrak{a}-\{0\}) / \mathfrak{r}^{\times}} \phi(\xi, 0) \operatorname{sgn}(N(\xi))^{k}|N(\xi)|^{-s}$. The construction of the Eisenstein measure is done in the following way: To each $\phi \in \mathscr{C}_{k}^{+}$, we associate linearly $\phi^{\circ} \in \mathscr{C}_{k}^{-}$as in [47] (3.1). Let $N: T \rightarrow \mathbb{Q}_{p}$ be $N\left(x, x^{\prime}, y, y^{\prime}\right)=N_{F_{p} / \mathbb{Q}_{p}}(x)$. Then $\mathbb{E}^{\text {n.ord }}: N^{-k} \phi \mapsto E_{k}\left(\phi^{\circ}\right) \in V$ gives a linear map into $V$ defined on functions on $T / \overline{\mathfrak{r}^{\times}}$which is locally a negative power of $N$. If $\phi$ is supported on $T^{\times} / \overline{\mathfrak{r}^{\times}}$, by (Q), the constant term of the $q$-expansion disappears, and hence $\mathbb{E}^{n . o r d}$ extends to a unique bounded measure with values in $V$ on $T^{\times} / \overline{\mathfrak{r}^{\times}}$. This measure is studied in [52] and [47]. Here instead of considering functions on $T^{\times}$, we consider functions $\phi$ on

$$
\mathscr{T}=\left(\mathfrak{r}_{p}^{\times} \times(\mathfrak{r} / \mathfrak{f}) \times(\mathfrak{r} / \mathfrak{G})\right) / \overline{\mathfrak{r}^{\times}} \text {for } \mathfrak{g} \supset \mathfrak{f} .
$$

The space $\mathscr{T}$ is a quotient of $T=\left\{\mathfrak{r}_{p} \times(\mathfrak{r} / \mathfrak{f})\right\} \times\left\{\mathfrak{r}_{p}^{\times} \times(\mathfrak{r} / \mathfrak{g})^{\times}\right\}$via $\pi:\left(x, x^{\prime}, y, y^{\prime}\right) \mapsto$ $\left(x, x^{\prime}, y^{\prime}\right)$. Then we still get a linear map $\mathbb{E}: N^{-k} \phi \mapsto E_{k}\left((\phi \circ \pi)^{\circ}\right) \in V$. However we can no longer expect the vanishing of the constant term of $\mathbb{E}(\phi)$. Actually the functional of constant term of $\mathbb{E}$ given by $N^{-k} \phi \mapsto$ (the constant term of $E_{k}\left(\phi^{\circ}\right)$ ) is the Deligne-Ribet pseudo measure [17] interpolating Hecke $L$-values of $F$ (see Section 6.7 in Chapter 6), which can have singularity. Thus, for each choice of an ideal $\mathfrak{x}$ prime to $p \mathfrak{f}$, we can define the ordinary Eisenstein measure $\mathbb{E}_{\mathfrak{c}, \mathfrak{c}}$ and the Eisenstein pseudo-measure $\mathbb{E}_{\mathfrak{c}}$ on $\mathscr{T}^{\times}$with values in $V\left(\mathfrak{c}, \mathfrak{f}^{2} ; \mathscr{V}\right)$ as follows:

$$
\mathbb{E}_{\mathfrak{c}, \mathfrak{c}}\left(N^{-k} \phi\right)=E_{k}\left(\phi^{\circ} ; \mathfrak{c}\right) \mid\left(1-\langle\mathfrak{x}\rangle N(\mathfrak{c})^{2}\right) \text { and } \mathbb{E}_{\mathfrak{c}}\left(N^{-k} \phi\right)=E_{k}\left(\phi^{\circ} ; \mathfrak{c}\right) .
$$

Here the word "pseudo-measure" just means that $\mathbb{E}_{\mathfrak{c}, \mathfrak{x}}\left(N^{-k} \phi\right)$ is a bounded measure.

\subsection{The $p$-ordinary Katz $p$-adic $L$-functions}

Let $(\varphi, \Sigma, S)$ be the triple studied in Chapter 7 for the CM quadratic extension $L / F$. Let $\mathbf{p}=\prod_{\mathbf{P} \in S} \mathbf{P}$. We define the $p$-ordinary Katz measure on $\mathscr{G}(C)=$ 
$C l_{L}\left(C \mathbf{p}^{\infty}\right)$ for a fixed ideal $C$ prime to $p$ and evaluate the integral of the $p$ adic avatars of $S$-ramified (but $S^{c}$-unramified) Hecke characters. We identify $F_{\mathbb{C}}$ with $\mathbb{C}^{\Sigma}$ via $a \mapsto\left(a^{\sigma}\right)_{\sigma \in \Sigma}$ for $a \in F$. For each fractional ideal $\mathfrak{U}$ prime to $C p$ in $L$, we take a test object $(A(\mathfrak{U}), \mathscr{P}(\mathfrak{U}), \omega(\mathfrak{U}), i(\mathfrak{U}))$ defined as follows: Over $\mathbb{C}$, $A(\mathfrak{U})(\mathbb{C})=F_{\mathbb{C}} / \Sigma(\mathfrak{U})$, where $\Sigma(\mathfrak{U})=\left\{\left(a^{\sigma}\right)_{s \in \Sigma} \mid a \in \mathfrak{U}\right\}$. We pick an element $\delta \in L$ as in $(\Delta 1-2)$ in Section 7.2. Then $\langle$,$\rangle induces \mathfrak{c}\left(\mathfrak{U} \mathfrak{U}^{c}\right)^{-1}$-polarization $\mathscr{P}(\mathfrak{U})$ on $A(\mathfrak{U})$. We define the level structure $i(\mathfrak{U}): F_{p} / \mathfrak{D}_{p}^{-1} \times\left(\mathfrak{f}^{2}\right)^{*} / \mathfrak{D}^{-1} \rightarrow A(\mathfrak{U})$ in exactly the same way as in [47] p.211. We recall the definition of $i(\mathfrak{U})$. We decompose $C=\mathfrak{F}_{c} \mathfrak{\Im}$ so that

(Ia) $\mathfrak{F}+\mathfrak{F}_{c}=\mathscr{R}, \quad \mathfrak{F}+\mathfrak{F}^{c}=\mathscr{R}, \quad \mathfrak{F}_{c}+\mathfrak{F}_{c}^{c}=\mathscr{R} \quad$ and $\quad \mathfrak{F}_{c} \supset \mathfrak{F}^{c}$,

(Ib) $\mathfrak{\Im}$ consists of ideals inert or ramified in $L / F$.

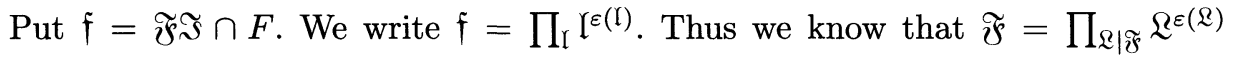
for primes $\mathfrak{Z}$ in $L$ for $\varepsilon(\mathfrak{L})=\varepsilon(\mathfrak{R} \cap F)$. We choose a prime element $\varpi_{\mathfrak{l}}$ for each prime $\mathfrak{l}$ dividing $p \mathfrak{f}$ in $F$ so that $\varpi_{\mathfrak{l}} \equiv 1 \bmod \mathfrak{f l}^{-\varepsilon(\mathfrak{l})}$ (in this formula, we agree to put $\varepsilon(\mathfrak{B})=0$ if $\mathfrak{B} \mid p)$ and $\varpi_{\mathfrak{l}}$ is prime to other prime $\mathfrak{l}^{\prime}$ dividing $p \mathfrak{f}$. We choose a differental idele $d$ of $F$ such that $d=d_{p \mathfrak{d}}$ and $d_{\mathfrak{q}}=(2 \delta)_{\mathfrak{Q}}$ for prime ideal $\mathfrak{Q} \mid \mathfrak{\Im}$ $(\mathfrak{q}=\mathfrak{a} \cap F)$. Then we have

$$
i(\mathfrak{U}): F_{p} / \mathfrak{D}_{p}^{-1} \times\left(\mathfrak{f}^{2}\right)^{*} / \mathfrak{D}^{-1} \rightarrow \mathbf{p}^{-\infty}(\mathfrak{\Im} \mathfrak{\Im})^{-2} \mathfrak{U} / \mathfrak{U} \quad \text { by } \quad x \mapsto d x
$$

which induces $i(\mathfrak{U}): F_{p} / \mathfrak{D}_{p}^{-1} \times\left(\mathfrak{f}^{2}\right)^{*} / \mathfrak{D}^{-1} \rightarrow A(\mathfrak{U})$. We can find a model

$$
\left(A(\mathfrak{U}), \mathscr{P}(\mathfrak{U}),\left.i(\mathfrak{U})\right|_{\left(\mathfrak{f}^{2}\right)^{*} / \mathfrak{D}-1}\right) / L^{\prime}
$$

for a finite extension $L^{\prime} / \mathbb{Q}$ which extends to an abelian scheme over the valuation ring $\mathscr{V}^{\prime}$ of $L^{\prime}$ corresponding to the embedding $i_{p}: L^{\prime} \hookrightarrow \overline{\mathbb{Q}}_{p}$, and there exists a nowhere vanishing differential $\omega(\mathfrak{U}) / \mathscr{V}^{\prime}$ on $A(\mathfrak{U}) / \mathscr{q}^{\prime}$. Moreover, defining $\mathscr{V}$ as the composite of $\mathscr{O}_{K_{0}}$ and $i_{p}\left(\mathscr{V}^{\prime}\right),(A(\mathfrak{U}), \mathscr{P}(\mathfrak{U}), \omega(\mathfrak{U}), i(\mathfrak{U}))$ extends to an abelian scheme over $\mathscr{V}_{0}=i_{p}^{-1}\left(i_{p}(\overline{\mathbb{Q}}) \cap \mathscr{V}\right)$. We put $\mathfrak{S}=\mathfrak{\Im}_{c} \cap F=\mathfrak{\mho}_{c}^{c} \cap F$ and $\mathfrak{i}=\mathfrak{\Im} \cap F$. Then we have homomorphisms

$$
\iota=\iota_{C}:\left\{\mathrm{r}_{p}^{\times} \times(\mathfrak{r} / \mathfrak{f})^{\times} \times(\mathfrak{r} / \mathfrak{H})^{\times}\right\} / \overline{\mathfrak{r}^{\times}} \rightarrow \mathscr{G}(C)
$$

induced by the natural inclusion of $F$ into $L$, where by $\iota$, the factor $\mathfrak{r}_{p}^{\times}$(resp. $(\mathfrak{r} / \mathfrak{f})^{\times}$, $\left.(\mathfrak{r} / \mathfrak{g})^{\times}\right)$is identified with $\mathscr{R}_{S}=\prod_{\mathbf{P} \in S} \mathscr{R}_{\mathbf{P}}^{\times}\left(\operatorname{resp} .(\mathscr{R} / \mathfrak{F})^{\times} \times(\mathfrak{r} / \mathfrak{i})^{\times},\left(\mathscr{R} / \mathfrak{\Im}_{c}\right)^{\times}\right)$. The morphism $\iota$ has finite kernel, and its cokernel is isomorphic to $\mathrm{Cl}^{-}(\mathfrak{\Im})$ which is the quotient of $C l_{L}(\mathfrak{I})$ by the natural image of $(\mathfrak{r} / \mathfrak{i})^{\times}$. We now choose a complete representative set $\left\{\mathfrak{U}_{j}\right\}$ for $C l^{-}(\mathfrak{S})$ consisting of fractional ideals prime to $p C C^{c}$. Let $[\mathfrak{U}]$ denote the class of $\mathfrak{U}$ in $\mathscr{G}(C)$. Then $\mathscr{G}(C)=\bigsqcup_{j \in C l^{-}(\mathfrak{I})} \operatorname{Im}(\iota)\left[\mathfrak{U}_{j}\right]^{-1}$. To define measures on $\mathscr{G}(C)$, we give a recipe of extending functions defined on $\mathscr{G}(C)$ to $\mathscr{T}$ which supports the Eisenstein measure. For each function $\phi$ on $\mathscr{G}(C)$ and 
index $j \in \mathrm{Cl}^{-}(\mathfrak{I})$, we define functions $\phi_{j}$ on $\mathscr{T}$ as follows: First on $\mathscr{T}^{\times}$, we put $\phi_{j}(x)=\phi\left(x\left[\mathfrak{U}_{j}\right]^{-1}\right)$. We extend this function to $\mathscr{T}$ by 0 outside $\mathscr{T}^{\times}$. We write the ideal of the polarization $\mathscr{P}\left(\mathfrak{A}_{j}\right)$ as $\mathfrak{c}_{j}$ and $E_{j}$ (resp. $E_{j, \mathfrak{x}}$ ) for the specialization of $\mathbb{E}_{\mathfrak{c}_{j}}$ (resp. $\left.\mathbb{E}_{\mathfrak{c}_{j}, \mathfrak{x}}\right)$ at the test object $\left(A\left(\mathfrak{U}_{j}\right), \mathscr{P}\left(\mathfrak{U}_{j}\right), i\left(\mathfrak{H}_{j}\right)\right)$. Then we define a measure $\mu_{\mathfrak{r}}$ on $\mathscr{G}(C)$ by

$$
\left(\mathscr{R}^{\times}: \mathfrak{x}^{\times}\right) \int_{\mathscr{G}(C)} \phi d \mu_{\mathfrak{x}}=\phi\left(\prod_{\mathfrak{L} \mid \mathfrak{F}} \varpi_{\mathfrak{Q}}^{\varepsilon(\mathfrak{R})} \prod_{\mathfrak{l} \mid \mathfrak{i}} \varpi_{\mathfrak{l}}^{\varepsilon(\mathfrak{l})}\right) \sum_{j} \int_{\mathscr{T} \times} \phi_{j} d E_{j, \mathfrak{x}} .
$$

Let $\chi: L_{\mathbb{A}}^{\times} / L^{\times} \rightarrow \mathbb{C}^{\times}$be a Hecke character of conductor $C \mathbf{p}^{e}$ for $\mathbf{p}^{e}=\prod_{\mathbf{P} \in S} \mathbf{P}^{e(\mathbf{P})}$ such that $\chi\left(x_{\infty}\right)=x_{\infty}^{\eta}$ with

$$
\eta=m_{0} \Sigma \text { for } m_{0}>0 .
$$

Then, in the same manner as in [47] Sections 4 and 5 , we can compute $\int \widehat{\chi} d \mu_{\mathfrak{x}}$, where $\widehat{\chi}$ is the $p$-adic avatar of $\chi$ defined on $\mathscr{G}(C)$. We just state the outcome:

Theorem 8.2.1 - Let the notation and the assumption be as above. Let $C$ be an integral ideal prime to $p$ in $L$. We put $W_{p}(\chi)=\prod_{\mathbf{P} \in S} N_{L / \mathbb{Q}}\left(\mathbf{P}^{-e(\mathbf{P})}\right) G\left(2 \delta ; \chi_{\mathbf{P}}\right)$ for the local Gauss sum $G\left(2 \delta ; \chi_{\mathbf{P}}\right)$ as in [47] (0.8). Then there exist a (unique) measure $\mu_{\mathfrak{x}}$ on $\mathscr{G}(C)$ for each ideal $\mathfrak{x}$ prime to $p C$ with values in $\mathscr{V}$ such that

$\frac{\int_{\mathscr{G}_{(}(C)} \hat{\chi} d \mu_{\mathfrak{x}}}{\Omega_{p}^{m_{0} \Sigma}(1-\chi(\mathfrak{x}))}=W_{p}(\chi) \frac{(-1)^{m_{0} t} \Gamma_{F}\left(m_{0} t\right)}{\sqrt{|D|} \Omega_{\infty}^{m_{0} \Sigma}} \prod_{\mathfrak{Q} \mid C}(1-\chi(\mathfrak{R})) \prod_{\mathbf{P} \in S}\left(1-\frac{\chi(\mathbf{P})^{-1}}{N(\mathbf{P})}\right) L(0, \chi)$ for all Hecke characters $\chi$ of conductor $C \mathbf{p}^{e}$ such that $\chi_{\infty}\left(x_{\infty}\right)=x_{\infty}^{m_{0} \Sigma}$ for $m_{0}>0$.

Since $1-\langle\mathfrak{c}\rangle$ has zero at the trivial character, we expect a pole of the $p$-adic $L$-function at the trivial character. By the construction of the Eisenstein measure, the value $\mathbb{E}_{\mathfrak{c}, \mathfrak{c}}(\mathbf{1})$ for the characteristic function $\mathbf{1}$ of $\mathfrak{r}_{p}^{\times}$is a constant, and we see from [12] Lemme 5.4 and its corollary that

$$
\mathbb{E}_{\mathfrak{c}, \mathfrak{x}}(\mathbf{1})=\log _{p}(\mathcal{N}(\langle\mathfrak{x}\rangle)) \frac{R_{p}}{w \sqrt{D}} \prod_{\mathbf{P} \in S}\left(1-\frac{1}{N_{L / \mathbb{Q}}(\mathbf{P})}\right) .
$$

\section{$8.3 p$-Ordinary $L$-function $L_{p}(\Phi)$}

Here we use the notation introduced in Chapter 7. Let $\lambda: h^{\text {ord }}\left(N p^{\infty} ; \mathcal{O}\right) \rightarrow \square$ be a $\Lambda^{\text {ord }}$-algebra homomorphism of CM type for a finite torsion-free $\Lambda^{\text {ord }}$-algebra $\llbracket$. Since $\lambda$ factors through $h^{\text {ord }}$, we see from $(\infty 1)$ in Section 7.1 of Chapter 7 that for a positive arithmetic point $P$, writing $\infty\left(\varphi_{P}\right)=m$, we have $m=-m_{0} \Sigma$ for $0<m_{0} \in \mathbb{Z}$. Thus $\varphi_{P}^{-1}$ satisfies the condition $(\infty)$ in Section 8.2. We then just put

$$
L_{p}(P, \Phi)=\int_{\mathscr{G}_{(C)}} \varphi_{P}^{-1} d \mu \quad \text { for } \quad \mu=(1-[\mathfrak{x}])^{-1} \mu_{\mathfrak{x}}
$$


Then assuming $I_{F a}=I_{R T}$ and using the complex functional equation, we see in the same manner as in (7.2.4) that

$$
\frac{L_{p}(P, \Phi)}{c_{p}^{+}\left(\Phi_{P}\right)}=\left(\mathscr{R}^{\times}: \mathfrak{r}^{\times}\right) N(\mathfrak{c})^{-1} \varphi_{P}(\mathfrak{c}) W\left(\varphi_{P}\right) \sqrt{D} E^{o r d}(P) \frac{\Gamma_{F}(m(P)) L\left(1, \varphi_{P}\right)}{c_{\infty}^{+}\left(\pi\left(\lambda_{P}\right)\right)}
$$

where $E^{\text {ord }}(P)=\prod_{\mathfrak{R} \mid C}\left(1-\varphi_{P}^{-1}(\mathfrak{Q})\right) \times \prod_{\mathbf{P} \in S}\left(1-\boldsymbol{\varphi}_{P}(\mathbf{P}) N(\mathbf{P})^{-1}\right)$. Thus we know from (Res) and (8.2.2) the following result:

Theorem 8.3.1 - Let the notation and the assumption be as above. We assume that $I_{F a}=I_{R T}$ and the Leopoldt conjecture for $F$ and $p$. Then $L_{p}(\Phi)$ defined as above satisfies Conjecture 4.6.1 (ii) in Chapter 4. Moreover $L_{p}(\Phi)$ is holomorphic everywhere (i.e. belongs to $\llbracket \widehat{\otimes}_{\widehat{O}} \mathrm{O}_{\Omega}$ ) if $\varphi$ is non-trivial on the torsion part of $G(C)$. If $\varphi$ is trivial on the torsion-part, it is holomorphic outside the unique point $P$ such that $\varphi_{P}=\mathrm{id}$, and it has a simple pole at $P$.

We can deduce from (Res) a precise residue formula for $L_{p}(\Phi)$. We will deduce the formula in the following paragraph in a bit different way from Theorem 6.3.2 in Chapter 6 .

\subsection{Another proof of Theorem 8.3.1}

There is another way of getting the residue formula in Theorem 8.3.1 from Theorem 6.3.2 in Chapter 6. We shall give an outline of the proof here supposing that $\varphi$ induces the identity character on the torsion part. To simplify the exposition, we assume that $C=1$ and write $\mathscr{G}$ for $\mathscr{G}(1)$. The idea of the proof is the comparison of the residues of two $p$-adic $L$-functions: one is the $p$-ordinary Katz $p$-adic $L$-function given in Theorem 8.3.1 and the other is the $p$-ordinary $p$-adic Rankin product $L$-function constructed in Theorem 6.3.2. We use the notation introduced in the previous sections. We decompose $\mathscr{G}=\Delta \times W_{L}$ for a finite group $\Delta$ and a torsion free subgroup $W_{L}$. Take a character $\psi: \Delta \rightarrow \overline{\mathbb{Q}}^{\times}$. Thus we have the associated projection $\psi_{*}: \mathcal{O}[[\mathscr{G}]] \rightarrow \Lambda_{L}=\mathbb{O}\left[\left[W_{L}\right]\right]$. We assume that $\varphi=\psi_{*}$ for the identity character $\psi$. Let $\mathscr{X}_{L}=\operatorname{Hom}_{\mathcal{O}-a l g}\left(\Lambda_{L}, \overline{\mathbb{Q}}_{p}\right)=\operatorname{Spec}\left(\Lambda_{L}\right)\left(\overline{\mathbb{Q}}_{p}\right)$. Then for each $P \in \mathscr{X}_{L}$, we have a continuous character $\varphi_{P}=P \circ \psi_{*}: \mathscr{H} \rightarrow \overline{\mathbb{Q}}_{p}^{\times}$. We call $\varphi_{P}^{-1}$ (or P) critical (of type $\Sigma$ or $S$ ) if, regarding $\mathscr{G}$ as a quotient of $L_{A}^{\times}$, we have $\varphi_{P}\left(x_{p}\right)=x_{p}^{-\eta}$ for $\eta \in \mathbb{Z}\left[I_{L}\right]$ such that

the $p$-type $\eta$ of $\varphi_{P}^{-1}$ as above is $m_{0} \Sigma$ for an integer $m_{0}>0$.

Then, there exists a complex avatar $\varphi_{P}: L_{\AA}^{\times} \rightarrow \mathbb{C}^{\times}$with $\infty$-type $-\eta$ such that $i_{\infty}^{-1}\left(\varphi_{P}(\mathfrak{a})\right)=i_{p}^{-1}\left(\varphi_{P}(\mathfrak{a})\right)$ for all ideals $\mathfrak{a}$ prime to $\mathbf{p}$. Let

$$
L\left(s, \varphi_{P}\right)=\sum_{\mathfrak{n}} \varphi_{P}(\mathfrak{n}) N_{L / \mathbb{Q}}(\mathfrak{n})^{-s}
$$


be the complex $L$-function. Write $L_{p}\left(\varphi_{P}\right)\left(\right.$ resp. $\left.L_{\infty}\left(\varphi_{P}\right)\right)$ for $\left(1-\varphi_{P}(\mathfrak{x})\right)^{-1} \int \varphi_{P} d \mu_{\mathfrak{x}}$ for the Katz measure $\mu_{\mathfrak{r}}$ in Theorem 8.2.1 (resp. $L\left(0, \varphi_{P}\right)$ ). Then we have

$$
\frac{L_{p}\left(\varphi_{P}^{-1}\right)}{\Omega_{p}^{m_{0} \Sigma}}=W_{p}\left(\varphi_{P}^{-1}\right) \prod_{\mathbf{P} \in S}\left(1-\varphi_{P}^{-1}(\mathbf{P}) N(\mathbf{P})^{-1}\right) \frac{(-1)^{m_{0}[F: \mathbb{Q}]} \Gamma_{F}\left(m_{0} t\right) L_{\infty}\left(\varphi_{P}^{-1}\right)}{\sqrt{|D| \Omega_{\infty}^{m_{0} \Sigma}}}
$$

We now want to compare $L_{p}$ and $D_{p}^{\text {ord }}(\pi(\lambda) \otimes \check{\pi}(\lambda))$ in Theorem 6.3.2 of Chapter 6 . Since $\mathbf{W}^{\text {ord }}$ is naturally a subgroup of finite index of $W_{L}, \Lambda_{L}$ is a finite flat algebra extension of $\Lambda^{\text {ord }}$. In the same manner as in [47] Section 6 , we have the $\theta$-measure $\theta: \mathscr{C}\left(\mathscr{G} ; \mathbb{O}_{\Omega}\right) \rightarrow S^{\text {ord }}\left(D_{L / F} p^{\infty} ; \mathcal{O}\right)$ given by

$$
\mathbf{a}(\mathfrak{n}, \theta(\phi))=\sum_{\substack{N_{L / F}(\mathfrak{m})=\mathfrak{n} \\ \mathfrak{m}+\mathbf{p}=\mathscr{R}}} \phi(\mathfrak{m}) .
$$

Then $\theta$ induces an algebra homomorphism $\theta^{*}: h^{\text {ord }}\left(D_{L / F} p^{\infty} ; \mathcal{O}\right) \rightarrow \mathscr{O}[[\mathscr{G}]]$ satisfying [47] (6.7b). By our assumption, $\lambda=\psi_{*} \circ \theta^{*}: h^{\text {ord }}\left(D p^{\infty} ; \mathcal{O}\right) \rightarrow \Lambda_{L}$. Then $\lambda$ is primitive in the sense of [34] Theorem 3.6. We pick two points $P, Q \in \mathscr{A}_{\Lambda_{L}}$. Let $m_{0} \Sigma$ and $m_{0}^{\prime} \Sigma$ be the infinity type of $\varphi_{P}^{-1}$ and $\varphi_{Q}^{-1}$, respectively. Then writing $\kappa(P)$ for the weight of the point $P$, we have

$$
m_{0}=[m(\kappa(P))] \quad \text { and } \quad n(\kappa(P))=0 .
$$

Then $\theta\left(\varphi_{P}\right)=f(P)$ for the $\Lambda_{L}$-adic form $f$ with Hecke eigenvalues given by $\lambda$; thus, $\theta\left(\varphi_{P}\right) \in S_{\kappa(P)}\left(D_{L / F} p^{\alpha} ; \mathcal{O}\right)$ for suitable $\alpha>0$. Now we write $\mathscr{K}$ for the Katz measure on $\mathbb{G}=C l_{L}\left(p^{\infty}\right)$ given in [52]. By the functional equation [52] Theorem (5.3.0), we have

$$
\mathscr{K}(\xi)=\mathcal{N}(\mathfrak{c})^{-1} \varphi(\mathfrak{c})^{-1}(\xi) \mathscr{K}\left(\xi^{*}\right) \quad\left(\Leftrightarrow N_{L / \mathbb{Q}}(\mathfrak{c})^{-1}[\mathfrak{c}]^{-1} \mathscr{K}^{*}=\mathscr{K}\right),
$$

where $\xi^{*}(\mathfrak{n})=\xi\left(\mathfrak{n}^{c}\right)^{-1} \mathcal{N}(\mathfrak{n})^{-1}$ for characters $\xi: \varphi \rightarrow \overline{\mathbb{Q}}_{p}^{\times}$and $a \mapsto a^{*}$ is the involution of $\mathscr{O}[[\mathbb{G}]]$ induced by $[\mathfrak{n}]^{*}=\mathcal{N}(\mathfrak{n})^{-1}\left[\mathfrak{n}^{-c}\right]$. Thus comparing the evaluation formula of Theorem 8.2.1 and that of the Katz measure (see [52] and [47] Theorem II), we get

$$
\mathscr{K}\left(\xi^{*}\right)=\mathcal{N}(\mathfrak{c}) \xi(\mathfrak{c}) K(\xi)=\left(\mathscr{R}^{\times}: \mathfrak{r}^{\times}\right) \mathcal{N}(\mathfrak{c}) \xi(\mathfrak{c}) \prod_{\mathbf{P} \in S}\left(1-\xi\left(\mathbf{P}^{c}\right)\right) \mu(\xi)
$$

for all character $\xi: \mathscr{G} / \Delta\left(=W_{L}\right) \rightarrow \overline{\mathbb{Q}}_{p}^{\times}$. In other words, for the projection $\rho: \mathbb{G} \rightarrow W_{L}$, we have

$$
\rho_{*} \mathscr{K}^{*}=\left(\mathscr{R}^{\times}: \mathrm{r}^{\times}\right) \mathcal{N}(\mathfrak{c})[\mathfrak{c}] \prod_{\mathbf{P} \in S}\left(1-\left[\mathbf{P}^{c}\right]\right) \mu .
$$


Let $L_{1}, L_{1}^{*}, L_{2}^{*} \in \mathbb{O}\left[\left[W_{L} \times W_{L}\right]\right]$ and $L^{-},\left(L^{-}\right)^{*} \in \mathcal{O}\left[\left[W_{L}\right]\right]$ be such that

$$
\begin{aligned}
& L_{1}(P, Q)=\mu_{\mathfrak{Y}}\left(\varepsilon_{P, Q}\right), \quad L_{1}^{*}(P, Q)=\mathscr{K}\left(\varepsilon_{P, Q}^{*}\right), L_{2}(P, Q)=\mathscr{K}\left(\varepsilon_{P, Q}^{\prime}\right), \\
& L_{2}^{*}(P, Q)=\mathscr{K}\left(\left(\varepsilon_{P, Q}^{\prime}\right)^{*}\right) \quad \text { and } \quad L^{-}(P)=\mathscr{K}\left(\varepsilon_{P}\right),\left(L^{-}\right)^{*}(P)=\mathscr{K}\left(\varepsilon_{P}^{*}\right)
\end{aligned}
$$

for all arithmetic points $(P, Q)$, where $\varepsilon_{P, Q}=\varphi_{P}^{-1} \varphi_{Q}, \varepsilon_{P, Q}^{\prime}=\varphi_{P}^{-1}\left(\varphi_{Q}\right)_{c}$ and $\varepsilon_{P}=\varphi_{P}^{-1}\left(\varphi_{P}\right)_{c}=\varepsilon_{P, P}^{\prime}$. As seen in [47] Section 8, these measures exist and are uniquely determined. Then in exactly the same manner as in [47] Section 8 , we have

Theorem 8.4.1 - Suppose that $\left.\varphi\right|_{\Delta}$ is the identity character. Then we have

$$
D_{p}^{o r d}(\pi(\lambda) \otimes \check{\pi}(\lambda))=\left(\mathscr{R}^{\times}: \mathfrak{r}^{\times}\right)\left(1-\langle\mathfrak{x}\rangle \otimes\langle\mathfrak{d}\rangle^{-1}\right)^{-1} \frac{U L_{1}^{*} L_{2}^{*}}{\Delta(L / F)\left(L^{-}\right)^{*}}
$$

for a unit $U$ in $\mathcal{O}_{\Omega}\left[\left[W_{L} \times W_{L}\right]\right]$, where $U(P, P)=1$ for all $P$ and $\Delta(L / F)=$ $\prod_{\mathfrak{q} \mid D_{L / F}}\left(1-N_{F / \mathbb{Q}}(\mathfrak{q})^{-1}\right) h(L) / h(F)$ for the class numbers $h(L)$ and $h(F)$ of $L$ and $F$.

Since $L_{2}^{*}(P, P)=\left(L^{-}\right)^{*}(P)$ and $L_{1}^{*}(P, Q)=\left(\mathscr{R}^{\times} \quad: \mathfrak{r}^{\times}\right) \mathcal{N}(\mathfrak{c})[\mathfrak{c}] \prod_{\mathbf{P} \in S}(1-$ $\left.\left[\mathbf{P}^{c}\right]\right) \mu_{\mathfrak{x}}\left(\varepsilon_{P, Q}\right)$, from the definition of $L_{p}$ in Section 8.3 and the residue formula of $D_{p}^{\text {ord }}$ in Theorem 6.3.2 of Chapter 6, again we conclude Theorem 8.3.1 and the following residue formula:

$$
\begin{aligned}
& \operatorname{Res}_{P=\mathrm{id}}\left(L_{p}(\Phi)\right) \\
& =\left(\mathscr{R}^{\times}: \mathfrak{r}^{\times}\right)^{-1} \frac{h(L) R_{p}}{2 \sqrt{D}} \prod_{\mathfrak{B} \mid p}\left(1-N_{F / \mathbb{Q}}(\mathfrak{B})^{-1}\right)=\frac{h(L) R_{S}}{w \sqrt{D}} \prod_{\mathbf{P} \in S}\left(1-N_{L / \mathbb{Q}}(\mathbf{P})^{-1}\right),
\end{aligned}
$$

where $w$ is the number of roots of unity in $L$, and the relation of the regulator $R_{p}$ with the $S$-adic regulator $R_{S}$ is given by the following formula:

$$
\frac{2 R_{S}\left(\mathscr{R}^{\times}: \mathfrak{r}^{\times}\right)}{w}=R_{p}
$$

while $\frac{2 R_{L, \infty}\left(\mathscr{R}^{\times}: \mathrm{r}^{\times}\right)}{2^{[F: \mathscr{Q}]} w}=R_{\infty}$ for the archimedean regulators. There is a canonical choice of sign of $\frac{R_{p}}{\sqrt{D}}$ (see [12] Section 5) given by ordering the columns of the regulator matrix and the discriminant matrix according to any fixed order of embeddings $i_{p} \sigma$ $(\sigma \in I)$.

\subsection{Representations isogenous to $\Phi$}

Here we analyze the singularity of $L_{p}(\phi)$ for $\phi$ isogenous to $\Phi$. Suppose that $F=\mathbb{Q}$ and $p>2$ for simplicity. Let $W_{L}=\mathbf{W}^{\text {ord }} \cong \mathbb{Z}_{p}$ and $W \cong \mathbb{Z}_{p}^{2}$ be the torsion-free part of $\mathscr{G}(C)$ and $\mathbb{G}=C l_{L}\left(C p^{\infty}\right)$. There are three elements $\Phi$ and $\Phi_{ \pm}$ 
in the isomorphism class of $\pi(\lambda)$ when $\varphi$ is trivial on the torsion-part of $\mathscr{G}(C)$. To describe these, we note that $\square$ in this case is given by $\mathcal{O}\left[\left[W_{L}\right]\right]$, and $\varphi$ is the inclusion

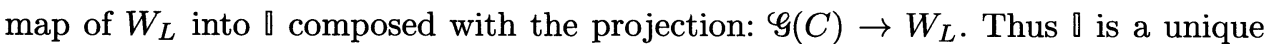
factorization domain, and for a prime divisor $P=(\pi), \Phi_{P}=\operatorname{Ind}_{L}^{\mathbb{Q}} \mathrm{id} \cong \mathrm{id} \oplus \chi$ for the quadratic character $\chi$ corresponding to $L / F$ and the identity character id. Let $W_{ \pm}=\left\{x \in W \mid c x c=x^{ \pm 1}\right\}$. Then for a generator $w$ of $W_{-}$, its projection to $W_{L}$ generates $W_{L}$, and $\pi=\varphi(w)-\varphi(c w c)$. We let these representations act on $V=\rrbracket^{2}$ so that

$$
V[c-1]=\left\{\left(\begin{array}{l}
x \\
0
\end{array}\right) \mid x \in \mathbb{Q}\right\} \quad \text { and } \quad V[c+1]=\left\{\left(\begin{array}{l}
0 \\
x
\end{array}\right) \mid x \in \mathbb{Q}\right\} .
$$

These representations have an exact sequence of $\mathscr{G}_{\mathbb{Q}^{-}}$-modules:

$$
0 \rightarrow V(\xi) \rightarrow V\left(\Phi_{P}\right) \rightarrow V(\psi) \rightarrow 0
$$

We can characterize $\phi \in\left\{\Phi_{+}, \Phi, \Phi_{-}\right\}$as follows:

\begin{tabular}{|c|c|c|c|}
\hline & $\Phi_{+}$ & $\Phi$ & $\Phi_{-}$ \\
\hline Sequence(8.8.1) & non-split & split & non-split \\
\hline$(\xi, \psi)$ & $(\chi$, id $)$ & $($ id,$\chi)$ & $($ id,$\chi)$ \\
\hline
\end{tabular}

\begin{tabular}{|c|c|c|c|c|c|}
\hline$\phi$ & $V_{p}^{+}(\phi)$ & $\alpha_{+}(\phi)$ & $\alpha_{-}(\phi)$ & $\operatorname{ord}_{P}\left(L_{p}(\phi)\right)$ & $\ell_{P}(\phi)$ \\
\hline$\Phi_{+}$ & $\left\{\left(\begin{array}{c}\pi x \\
-x\end{array}\right) \mid x \in \mathbb{Q}\right\}$ & non-unit: $\pi$ & unit & 2 & 2 \\
\hline$\Phi$ & $\left\{\left(\begin{array}{c}-x \\
x\end{array}\right) \mid x \in \mathbb{Q}\right\}$ & unit & unit & 1 & 1 \\
\hline$\Phi$ & $\left\{\left(\begin{array}{c}-x \\
\pi x\end{array}\right) \mid x \in \mathbb{}\right\}$ & unit & non-unit: $\pi$ & 1 & 0 \\
\hline
\end{tabular}

Then on $V_{p}^{+}, \mathscr{H}=\mathscr{G}_{L}$ acts via $\varphi_{c}$, and we have $V_{p}^{+}(\phi)$ and $\alpha_{ \pm}(\phi)$ as follows:

In particular, for $\Phi$ and $\Phi_{+}$, the principle $\left(\mathrm{AT}_{p}\right)$ holds in strict sense (i.e. $(\mathrm{G})$ in Section 4.4 of Chapter 4 holds), but for $\Phi_{-}$, only weaker $\left(\mathrm{AT}_{p}\right)$ holds.

Let $\nu: \mathscr{G}_{\mathbb{Q}} \rightarrow \mathcal{O}[[\Gamma]]^{\times}$be the universal cyclotomic character as in Section 4.5 of Chapter 4 . Then we can think about $\Phi_{\text {? }} \otimes \nu$. Since $W \cong W_{L} \times \Gamma$ identifying $\Gamma$ with $W_{+}$, again using the (non-ordinary) Katz $p$-adic $L$-function, we can construct $L_{p}(\Phi \otimes \nu)$. Since $\alpha_{ \pm}\left(\Phi_{?} \otimes \nu\right)=\alpha_{ \pm}\left(\Phi_{?}\right) \in \mathcal{O}\left[\left[W_{L}\right]\right] \subset \mathcal{O}\left[\left[W_{L} \times \Gamma\right]\right]$, by the effect of Euler factor at $p, \operatorname{ord}_{P}\left(L_{p}(\Phi \otimes \nu)\right)=0$ at the prime divisor $\mathbf{P}: \mathcal{O}\left[\left[W_{L} \times \Gamma\right]\right] \rightarrow$ $\mathcal{O}[[\Gamma]]$ induced by the projection to $\Gamma$. Thus we have $\operatorname{ord}_{\mathbf{P}}\left(L_{p}\left(\Phi_{+} \otimes \nu\right)\right)=1$ and $\operatorname{ord}_{\mathbf{P}}\left(L_{p}\left(\Phi_{-} \otimes \nu\right)\right)=0$. On the other hand, $\left(\Phi_{+} \otimes \nu\right)_{\mathbf{P}}$ has a non-split exact sequence

$$
0 \rightarrow \chi \nu \rightarrow\left(\Phi_{+} \otimes \nu\right)_{\mathbf{P}} \rightarrow \nu \rightarrow 0
$$

This shows, on the contrary to the ordinary case, for $\Phi_{-} \otimes \nu$ and $\Phi \otimes \nu$, the assertion (G) holds, but for $\Phi_{+} \otimes \nu$, the principle $\left(\mathrm{AT}_{p}\right)$ fails to hold. 


\section{Bibliography}

[1] Y. André, $p$-adic Betti lattices, In " $p$-adic Analysis", Lecture notes in Math. 1454 (1989), 23-63

[2] D. Blasius, A p-adic property of Hodge classes of abelian varieties, Proc. Symp. Pure Math. 55 Part 2 (1994), 293-308

[3] $\longrightarrow$ Period relations and critical values of $L$-functions, preprint, 1989

[4] On the critical values of Hecke L-series, Ann. of Math. 124 (1986), 23-63

[5] - Appendix to Orloff Critical values of certain tensor product L-functions, Invent. Math. 90 (1987), 181-188

[6] D. Blasius and J. D. Rogawski, Motives for Hilbert modular forms, Inventiones Math. 114 (1993), 55-87

[7] S. Bloch and K. Kato, L-functions and Tamagawa numbers of motives, Progress in Math. (Grothendieck Festschrift 1) 86 (1990), 333-400

[8] N. Bourbaki, Algébre commutative, Hermann Paris, 1961-65

[9] H. Carayol, Sur les représentations $\ell$-adiques associées aux formes modulaires de Hilbert, Ann. Sci. Éc. Norm. Sup. 4-th series, 19 (1986), 409-468

[10] Formes modulaires et représentations galoisiennes à valeurs dans un anneau local compact, Contemporary Math. 165 (1994), 213-237

[11] W. Casselman, On some results of Atkin and Lehner, Math. Ann. 201 (1973), 301-314

[12] P. Colmez, Résidu en $s=1$ des fonctions zêta $p$-adiques, Inventiones Math. 91 (1988), 371-389

[13] Fonctions zêta $p$-adiques en $s=0$, J. reine angew. Math. 467 (1995), 89-107

[14] P. Colmez and L. Schneps, p-adic interpolation of special values of Hecke L-functions, Compositio Math. 82 (1992), 143-187 
[15] P. Deligne, Valeurs des fonctions $L$ et périodes d'intégrales, Proc. Symp. Pure Math. 33 (1979), part 2, 313-346

[16] Hodge cycles on abelian varieties, Lecture Notes in Math. 900 (1982), 9-100

[17] P. Deligne and K. A. Ribet, Values of abelian L-functions at negative integers over totally real fields, Invent. Math. 59 (1980), 227-286

[18] P. Deligne and J.S. Milne, Tannakian categories, Lecture notes in Math. 900 (1982), 101-228

[19] K. Doi, H. Hida and H. Ishii, Discriminant of Hecke fields and the twisted adjoint L-values for $G L(2)$, preprint, 1996

[20] G. Faltings, Crystalline cohomology and p-adic Galois representations, Proc. JAMI inaugural Conference, supplement to Amer. J. Math. (1988), 25-80

[21] —

[22] J.-M. Fontaine, Sur certains types de représentations p-adiques du group de Galois d'un corps local; construction d'un anneau de Barsotti-Tate, Ann. of Math. 115 (1982), 529-577

[23] J.-M. Fontaine, Modules galoisiens, modules filtrés et anneaux de BarsottiTate, Astérisque 65 (1979), 3-80

[24] J.-M. Fontaine and W. Messing, p-adic periods and p-adic étale cohomology, Contemporary Math. 67 (1987), 179-207

[25] K. Fujiwara, Deformation rings and Hecke algebras in totally real case, preprint, 1996

[26] S. Gelbart and H. Jacquet, A relation between automorphic representations of $G L(2)$ and $G L(3)$, Ann. Scient. Ec. Norm. Sup. 4-th series 11 (1978), 471-542

[27] R. Gillard, Relations monomiales entre périodes p-adiques, Invent. Math. 93 (1988), 355-381

[28] R. Greenberg, Iwasawa theory and p-adic deformations of motives, Proc. Symp. Pure Math. 55 (1994) Part 2, 193-223

[29] , Iwasawa theory for $p$-adic representations, Adv. Studies Pure Math. 17 (1989), 97-137

[30] M. Harris, Period invariants of Hilbert modular forms I: Trilinear differential operators and L-functions, Lecture note in Math. 1447 (1990), 155-202

[31] $L$ - Lunctions of $2 \times 2$ unitary groups and factorization of periods of Hilbert modular forms, J. Amer. Math. Soc. 6 (1993), 637-719 
[32] M. Harris and J. Tilouine, p-adic measures and square roots of triple product L-functions, preprint, 1994

[33] H. Hida, Elementary Theory of L-functions and Eisenstein series, LMSST 26, Cambridge University Press, 1993

[34] On p-adic Hecke algebras for $G L_{2}$ over totally real fields, Ann. of Math. 128 (1988), 295-384

[35] - On nearly ordinary Hecke algebras for GL(2) over totally real fields, Adv. Studies in Pure Math. 17 (1989), 139-169

[36] _ Iwasawa modules attached to congruences of cusp forms, Ann.

Sci. Ec. Norm. Sup. 4-éme série 19 (1986), 231-273

[37] — A p-adic measure attached to the zeta functions associated with two elliptic modular forms I, Inventiones Math. 79 (1985), 159-195; II, Ann. l'Institut Fourier 38 (1988), 1-83

[38] Nearly ordinary Hecke algebras and Galois representations of several variables, Proc. JAMI inaugural Conference, supplement to Amer. J. Math. (1988), 115-134

[39] _ Modules of congruence of Hecke algebras and L-functions associated with cusp forms, Amer. J. Math. 110 (1988), 323-382

[40] Le produit de Petersson et de Rankin p-adique, Sém. Théorie des Nombres, 1988-89, 87-102

[41] - On p-adic L-functions of $G L(2) \times G L(2)$ over totally real fields, Ann. l'Institut Fourier 41 No.2 (1991), 311-391

[42] - On the critical values of L-functions of $G L(2)$ and $G L(2) \times$ GL(2), Duke Math. J. 74 (1994), 431-529

[43] — Congruences of cusp forms and special values of their zeta functions, Inventiones Math. 63 (1981), 225-261

[44] Galois representations into $G L_{2}\left(\mathbb{Z}_{p}[[X]]\right)$ attached to ordinary cusp forms, Inventiones Math. 85 (1986), 545-613

[45] - On Selmer groups of adjoint modular Galois representations, Number Theory, Paris, LMS lecture notes series, 1996

[46] Non-critical values of adjoint L-functions for $S L(2)$, preprint, 1997

[47] H. Hida and J. Tilouine, Anti-cyclotomic Katz p-adic L-functions and congruence modules, Ann. Scient. Ec. Norm. Sup. 26 (1993), 189-259

[48] On the anticyclotomic main conjecture for CM fields, Inventiones Math. 117 (1994), 89-147 
[49] H. Hida, J. Tilouine, and E. Urban, Adjoint modular Galois representations and their Selmer groups, Proc. NAS. 1997

[50] Luc Illusie, Cohomologie de de Rham et cohomologie étale p-adique, Séminaire Bourbaki, 1989-90 no. 726

[51] H. Jacquet, Automorphic forms on $G L(2)$, II, Lecture notes in Math. 278, Springer, 1972

[52] N. M. Katz, p-adic L-functions for CM fields, Inventiones Math. 49 (1978), 199-297

[53] K. Kitagawa, On standard p-adic L-functions of families of elliptic cusp forms, Contemporary Math. 165 (1994), 81-110

[54] B. Mazur, Deforming Galois representations, in "Galois group over $\mathbb{Q}$ ", MSRI publications 16, (1989), 385-437

[55] B. Mazur and J. Tilouine, Représentations Galoisiennes, différentielles de Kähler et "conjectures principales", Publ. IHES 71 (1990), 65-103

[56] A. A. Panchishkin, Admissible non-archimedean standard zeta functions associated with Siegel modular forms, Proc. Symp. Pure Math. 55 Part 2 (1994), 251-292

[57]

Produits triples des formes modulaires et leur interpolation p-adique par la méthode d'Amice-Vélu, preprint 1993

[58] B. Perrin-Riou, Fonction L p-adiques des représentations p-adiques, Astérisque 229 (1995)

[59] - Variation de la fonction L p-adique par isogénie, Adv. Studies Pure Math. 17 (1989), 347-358

[60] - Représentations p-adiques, périodes et fonctions $L$ p-adiques, Séminaire de théorie des nombres, Paris (1987-88), 213-258

[61] J.-P. Serre, Abelian $\ell$-adic representations and elliptic curves, Benjamin, 1968

[62] —_, Groupes algébriques associés aux modules de Hodge-Tate, Astérisque 65 (1979), 155-188

[63] — Propriétés conjecturales des groupes de Galois motiviques et des représentations $\ell$-adiques, Proc. Symp. Pure Math. 55, Part 1, 377-400

[64] G. Shimura, Introduction to the arithmetic theory of automorphic functions, Iwanami-Shoten and Princeton Univ. Press, 1971

[65] - The special values of the zeta functions associated with Hilbert modular forms, Duke Math. J. 45 (1978), 637-679 
[66] On the critical values of certain Dirichlet series and the periods of automorphic forms, Inventiones Math. 94 (1988), 245-305

[67] On the fundamental periods of automorphic forms of arithmetic type, Inventiones Math. 102 (1990), 399-428

[68] J. Tate, $p$-Divisible groups, Proc. of Conference on local fields, Driebergen 1966, 158-183

[69] R. Taylor, On Galois representations associated to Hilbert modular forms, Inventiones Math. 98 (1989), 265-280

[70] R. Taylor and A. Wiles, Ring theoretic properties of certain Hecke modules, Ann. of Math. 142 (1995), 553-572

[71] J. Tilouine, Sur la conjecture principale anticyclotomique, Duke. Math. J. 59 (1989), 629-673

[72] — Deformation of Galois representations and Hecke algebras, Publ. Mehta Res. Inst., Narosa Publ., Delhi, 1996

[73] A. Wiles, Modular elliptic curves and Fermat's last theorem, Ann. of Math. 142 (1995), 443-551

[74] H. Yoshida, On the zeta functions of Shimura varieties and periods of Hilbert modular forms, Duke Math. J. 75 (1994), 121-191

[75] — On a conjecture of Shimura concerning periods of Hilbert modular forms, Amer. J. Math. 117 (1995), 1019-1038 


\section{A. Correction to [41]}

page $317(0.4 \mathrm{~b}): \Phi_{P}^{\prime}$

should read $\psi_{P}^{\prime}$

page 317 line 8 from the bottom:

$$
G\left(\xi_{\mathfrak{p}}^{\prime-1} \psi_{\mathfrak{p}}^{\prime}-1\right) \quad \text { should read } \quad G\left(\xi_{\mathfrak{p}}^{\prime-1} \psi_{\mathfrak{p}}^{\prime-1}\right)
$$

page $318(*): L\left(0, M\left(\mathbf{f}_{P}\right) \otimes M\left(\mathbf{g}_{Q}\right)^{\vee}\right)$ should read $L\left(1, M\left(\mathbf{f}_{P}\right) \otimes M\left(\mathbf{g}_{Q}\right)^{\vee}\right)$

page 319 lines 2, 15 from the top:

$$
M\left(\mathbf{f}_{P}\right) \quad \text { should read } \quad M\left(\mathbf{f}_{P}\right)(1) \text { (the Tate twist) }
$$

page 320 lines $3,4,8$ from the bottom:

$$
\Lambda(0, M(P, Q)) \quad \text { should read } \quad \Lambda(1, M(P, Q))
$$

page 330 line 11 from the bottom:

$$
Z(N) \times F_{\mathbf{A}}^{\times} / \overline{F^{\times} U_{F}\left(N p^{\infty}\right) F_{\infty+}^{\times}} \quad \text { should read } \quad Z(N)=F_{\mathbf{A}}^{\times} / \overline{F^{\times} U_{F}\left(N p^{\infty}\right) F_{\infty+}^{\times}}
$$

page 330 line 2 from the bottom:

$$
\text { q outside } N
$$

should read q dividing $N$

page 331 line 2 from the bottom:

$$
t_{p}(\mathscr{V}) \quad \text { should read } \iota_{p}(\mathcal{V})
$$

page 333 line 9 from the top: $\mathbf{M}_{k, w} \quad$ should read $\quad \overline{\mathbf{M}}_{k, w}$

page 333 line 8 from the bottom:

$$
f \notin \widehat{\mathfrak{r}} \cap F_{\mathbf{A}_{f}}^{\times} \quad \text { should read } \quad f \in \widehat{\mathfrak{r}} \cap F_{\mathbf{A}_{f}}^{\times}
$$

page 340 line 3 from the top:

$$
\mathbf{e}_{F}(-\xi x) \quad \text { should read } \mathbf{e}_{F}(\xi x)
$$

page 341 line 4 from the top:

$$
D^{(1+2 s) /} \quad \text { should read } \quad D^{(1+2 s) / 2}
$$


page 348 line 16 from the top

$$
|D|^{1+m(Q)-(P)}
$$

page 363 (6.8): $\sum_{\substack{\gamma \in \mathfrak{r}^{\times} \backslash F^{\times} \\ \xi \in F}}$

should read $|D|^{1+m(Q)-m(P)}$

should read $\quad \sum_{\substack{\gamma \in \mathfrak{r}^{\times} \backslash F^{\times} \\ \delta \in F}}$

page 366 line 3 from the top: $x, \tau(m)$ should read $x \tau(m)$

page 374 (8.1a):

$\mathbf{a}_{p}\left(y, \int_{G(L)} \phi\left(w^{-1}, z\right) w^{-t} \mathcal{N}(z) d E\right) \quad$ should read

$\mathbf{a}_{p}\left(y, \int_{G(L)} \phi\left(w^{-1}, z\right) \mathcal{N}(z) d E\right)$

$\mathbf{a}_{0, p}\left(y, \int_{G(L)} w^{-t-r} \phi(z) \mathcal{N}(z) d E\right) \quad$ should read

$\mathbf{a}_{0, p}\left(y, \int_{G(L)} \phi(z) \mathcal{N}(z) d E\right)$ 


\section{B. List of Symbols}

Chapter 1

$T_{n}$ : above (A1)

$\mathscr{G}_{F}, \mathscr{G}_{F_{\mathfrak{\beta}}}:$ above (A1)

I: above (A1)

$M_{P}, E_{P}:$ in $(\mathrm{A} 1)$

$V_{\mathfrak{B}}^{ \pm}:$in $\left(\mathrm{A} 2_{ \pm}\right)$

\section{Chapter 2}

$T$ : Section 2.1

G, $\mathbf{G}^{\text {ord }}$ : Section 2.1

$\widehat{\Gamma}_{1}(N)$ : Section 2.1

$\mathscr{X}, \mathscr{X}^{\text {ord }}$ : Section 2.1

$\mathscr{A}, \mathscr{A}^{\text {ord }}$ : Section 2.1

$m(\kappa), n(\kappa)$ : Section 2.1

$\kappa, \varepsilon_{\kappa}$ : Section 2.1

$U_{0}\left(p^{\alpha}\right), U_{0}\left(p^{\alpha}\right), U_{1}^{1}\left(p^{\alpha}\right)$ : Section 2.2

$\langle a\rangle$ : Section 2.2

$S_{\kappa}(V ; A), M_{\kappa}(V ; A)$ : Sections 2.3-2.4

$\mathbf{a}_{p}(y, f)$ : Section 2.3

$\mathbb{T}(y), \mathbb{T}(\mathfrak{l})$ : Section 2.3

$h_{\kappa}^{\text {ord }}, h_{\kappa}^{\text {n.ord }}, h_{\kappa}\left(N p^{\infty} ; \mathcal{O}\right)$ : Section 2.5

$\mathbf{W}, \mathbf{W}^{\text {ord }}$ : Section 2.5

$\langle$,$\rangle : Section 2.6$

$S_{\kappa, U}^{n . o r d}, S_{\kappa, U}^{\text {ord }}$ : Section 2.6

$M_{\kappa}\left(V_{\infty} ; \mathcal{O}\right), S_{\kappa}\left(V_{\infty} ; \mathcal{O}\right)$ : Section 2.6

$f(P)$ : Section 2.7

$\mathscr{A}_{0}, \mathscr{X}_{\mathbb{1}}$ : Section 2.7

$\pi(\lambda), \pi\left(\lambda_{P}\right):$ Section 2.8

$\bar{Z}, \bar{Z}(N)$ : Section 2.9

$h^{\text {ord }}(\psi), h^{\text {n.ord }}(\psi)$ : Section 2.9
$C_{0}(\lambda, \llbracket), C_{1}(\lambda ; \square)$ : Section 2.9

$S_{\kappa}(N p ; \mathbb{C})[\psi]$ : Section 2.10

$\Omega\left( \pm, \lambda_{P} ; A\right), L\left(n, \psi_{P} ; A\right)$ : Section 2.10

$\operatorname{Sel}(A d(\rho))$ : Section 2.10

\section{Chapter 3}

$L_{\iota}(s, \varphi), L(s, M)$ : Section 3.1

$H_{\mathfrak{p}}(M)$ : Section 3.1

$\Gamma(s, \varphi)$ : Section 3.1

$I_{X, \sigma}, I_{X, \sigma}^{ \pm}$: Section 3.1

$\mathscr{F}^{ \pm}(M), H_{B}^{ \pm}\left({ }^{\sigma} M\right)$ : Section 3.1

$H_{D R}^{ \pm}$: Section 3.1

$\delta_{X, \sigma}(\varphi)$ : Section 3.1

$c_{X, \iota}^{ \pm}(\varphi), c_{X, \iota, \sigma}^{ \pm}(\varphi)$ : Section 3.1

$c_{\iota}^{ \pm}(\varphi), c_{\iota, \sigma}^{ \pm}(\varphi)$ : Section 3.1

$I_{i_{p} \sigma, D R}, I_{\iota \sigma, e t}, I_{i_{p} \sigma, H T}$ : Section 3.2

$S_{F}$ : Section 3.2

$c_{p, \sigma, \iota}^{ \pm}(\varphi), c_{p, \iota}^{ \pm}(\varphi)$ : Section 3.2

\section{Chapter 4}

$V[c \pm 1]$ : Section 4.1

$\Phi_{ \pm}(\tau), \beta_{ \pm}(\tau)$ : Section 4.1

$L_{S, i_{\infty}}(P, \varphi), \mathcal{N}:$ Section 4.2

$L_{S}(\varphi), \alpha_{ \pm}(\varphi)$ : Section 4.3

$\Omega_{\infty}^{ \pm}, \Omega_{p}^{ \pm}$: Section 4.3

$\varphi^{*}, \ell_{P}(\varphi)$ : Section 4.4

$\operatorname{ord}_{P}$ : Section 4.4

\section{Chapter 5}

$c_{X, J}^{ \pm}(M), \delta_{X, J}(M)$ : Section 5.1

$A d(M)$ : Section 5.2 
$m_{i}(\sigma), n_{i}(\sigma)$ : Section 5.3

type $(A, T, C)$ : Section 5.4

\section{Chapter 6}

$L\left(\varphi_{P} \otimes \check{\rho}_{Q}\right)$ : Section 6.1

$D_{p}(\pi(\lambda) \otimes \check{\pi}(\mu))$ : Section 6.2

$\kappa(P)$ : Section 6.3

$D_{p}^{\text {ord }}(\pi(\lambda) \otimes \check{\pi}(\mu))$ : Section 6.3

$\chi_{Q}, \psi_{P}$ : Section 6.4

$L\left(s, \lambda_{P} \otimes \check{\mu}_{Q}\right)$ : Section 6.4

$h^{u}(x)$ (unitarization): Section $6.4(\mathrm{U})$

$\mathfrak{P}^{\alpha}, \tau\left(n \varpi^{\alpha}\right)$ : Section 6.4

$C_{0}(P, Q)$ : Section 6.5 Theorem 6.5.1

$(,)_{\alpha}$ : Section 6.5 Theorem 6.5.1

$W(\phi)$ : Section 6.5 (FE1), (W1)

$W_{0}(P, Q)$ : Section $6.5(\mathrm{~W} 2)$

$\widehat{\Gamma}_{0}(\mathfrak{m})$ : Section 6.6

$E^{\prime}(P, Q), S(P)$ : Section 6.6,

Lemma 6.6.1

$U_{F}\left(L p^{\alpha}\right)$ : Section 6.7
$Z(L), C l_{F}(N), C l_{F}^{0}(N)$ : Section 6.7

$\mathscr{C}(T ; \mathcal{O}), \zeta_{\mathrm{r}, L}:$ Section 6.7

$E_{\mathfrak{x}, L}$ : Section 6.7

$E(\xi)$ : Section 6.7

$E * \varphi$ : Section 6.8

$E *_{\lambda} \varphi$ : Section 6.9

\section{Chapter 7}

$L_{S}(\Phi \otimes \check{\rho})$ : Section 7.1

$\Sigma$ : Section 7.1

$\mathscr{C}, I_{F a}, I_{R T}$ : Section 7.2

$c_{p}^{ \pm}\left(\Phi_{P} ; I_{X}\right)$ : Section 7.2

$\Omega_{p}, \Omega_{\infty}$ : Section 7.2

$L_{p}(A d(\Phi))$ : Section 7.3

\section{Chapter 8}

$\mathbb{E}_{\mathrm{c}, \mathfrak{x}}, \mathbb{E}_{\mathfrak{c}}$ : Section 8.1

$\varphi(C), \mathbf{p}$ : Section 8.2

$\mu_{\mathfrak{y}}, C l^{-}(\Im)$ : Section 8.2

$W_{p}(\chi)$ : Section 8.2,Theorem 8.2.1

$\mu$ : Section 8.3 